Check for updates

Cite this: Chem. Soc. Rev., 2021, 50, 3355

Received 1st October 2020

DOI: 10.1039/d0cs00384k

rsc.li/chem-soc-rev

\section{Radiolabelling of nanomaterials for medical imaging and therapy}

\author{
Juan Pellico, (D) $\dagger$ Peter J. Gawne (D) $\dagger$ and Rafael T. M. de Rosales (D) *
}

\begin{abstract}
Nanomaterials offer unique physical, chemical and biological properties of interest for medical imaging and therapy. Over the last two decades, there has been an increasing effort to translate nanomaterialbased medicinal products (so-called nanomedicines) into clinical practice and, although multiple nanoparticle-based formulations are clinically available, there is still a disparity between the number of pre-clinical products and those that reach clinical approval. To facilitate the efficient clinical translation of nanomedicinal-drugs, it is important to study their whole-body biodistribution and pharmacokinetics from the early stages of their development. Integrating this knowledge with that of their therapeutic profile and/or toxicity should provide a powerful combination to efficiently inform nanomedicine trials and allow early selection of the most promising candidates. In this context, radiolabelling nanomaterials allows whole-body and non-invasive in vivo tracking by the sensitive clinical imaging techniques positron emission tomography (PET), and single photon emission computed tomography (SPECT). Furthermore, certain radionuclides with specific nuclear emissions can elicit therapeutic effects by themselves, leading to radionuclide-based therapy. To ensure robust information during the development of nanomaterials for PET/SPECT imaging and/or radionuclide therapy, selection of the most appropriate radiolabelling method and knowledge of its limitations are critical. Different radiolabelling strategies are available depending on the type of material, the radionuclide and/or the final application. In this review we describe the different radiolabelling strategies currently available, with a critical vision over their advantages and disadvantages. The final aim is to review the most relevant and up-to-date knowledge available in this field, and support the efficient clinical translation of future nanomedicinal products for in vivo imaging and/or therapy.
\end{abstract}

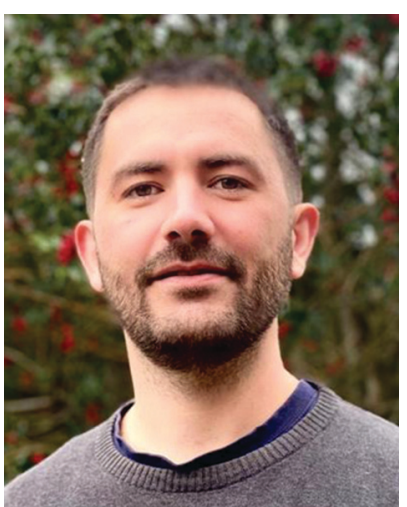

Juan Pellico
Juan Pellico Sáez obtained his PhD degree in Chemistry from the Complutense University of Madrid (UCM) in 2016. He then obtained a grant to conduct postdoctoral research in the Spanish Centre for Cardiovascular Research (CNIC). In 2018, he moved to the University of Oxford as a Postdoctoral Research Associate (PDRA). He joined to the group of $\mathrm{Dr}$ Rafael T. M. de Rosales at King's College London in 2019 as a PDRA. His main area of interest combines novel particulate PET tracers with the application of nanotechnology in biomedicine to develop a new generation of imaging agents for multimodal molecular imaging applications.

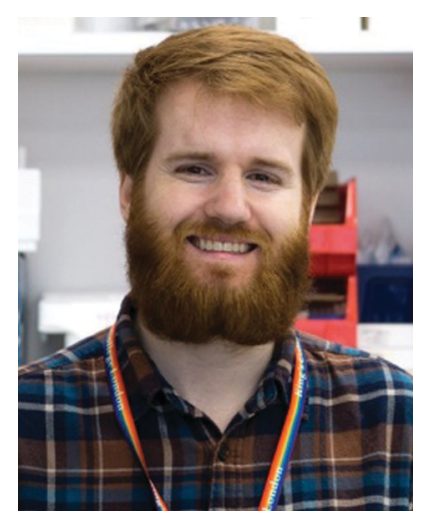

Peter J. Gawne
Peter Gawne received his Masters in Chemistry from the University of Hull, before joining the Medical Imaging CDT at King's College London and Imperial College London in 2015; obtaining a Masters of Research in Medical Imaging Science, followed by his PhD in Radiochemistry at King's College London-under the supervision of Dr Rafael T. M. de Rosales. He is currently continuing his work as a Postdoctoral Research Associate focusing on the radiolabelling of cells and nanomedicines. 


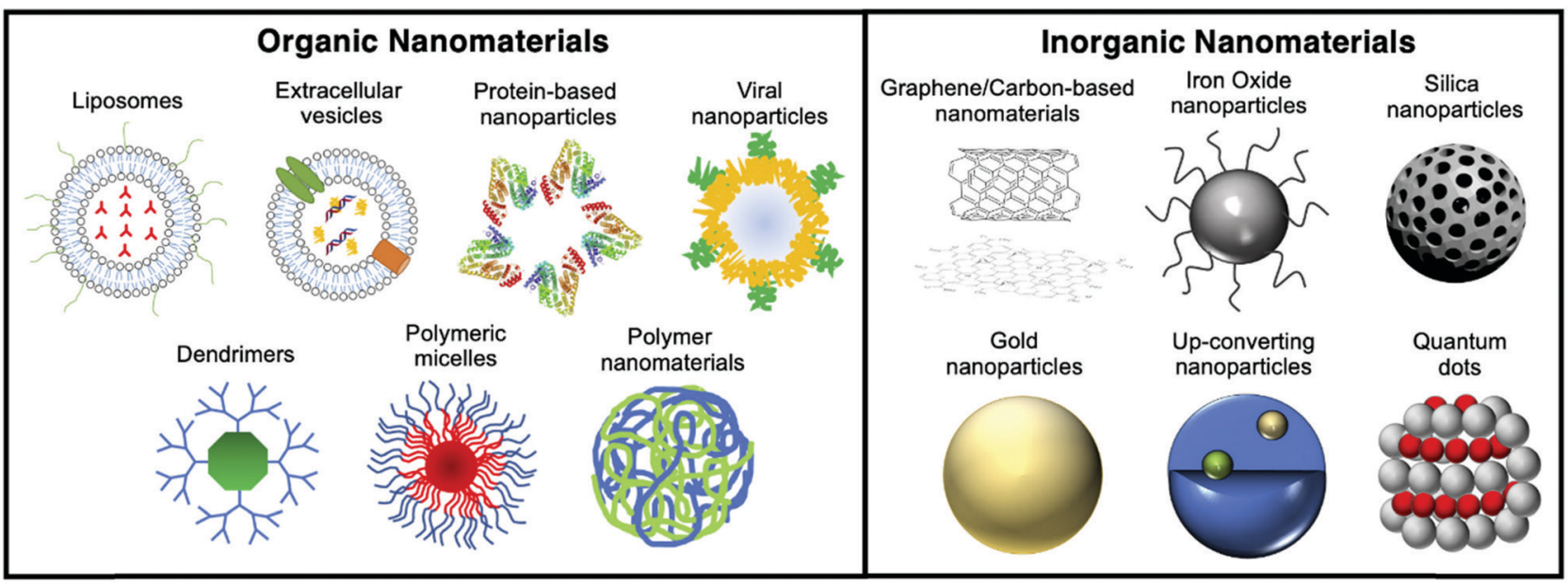

Fig. 1 Schematic showing the various organic and inorganic nanomaterials discussed in this review.

\section{Introduction}

Materials at the nanometric scale (i.e. with at least one dimension below $100 \mathrm{~nm}$ ) have emerged in the last 20 years as tools with several unique applications in imaging, diagnosis and treatment in medicine. Since then, the use of nanomaterials in medicine (nanomedicine) has evolved tremendously, with an increasing number of examples that overcome previously unmet medical needs (Fig. 1). ${ }^{1}$ The size-dependent optical, magnetic, and/or electronic properties of nanomaterials offer multiple possibilities in different fields of application. In addition, the tuneable nature of their physicochemical properties, pharmacokinetics and biodistribution has allowed the development of improved drug delivery systems, where the formulation is mainly driven towards the malignant areas rather than healthy areas, decreasing undesirable side effects and boosting therapeutic efficacy.

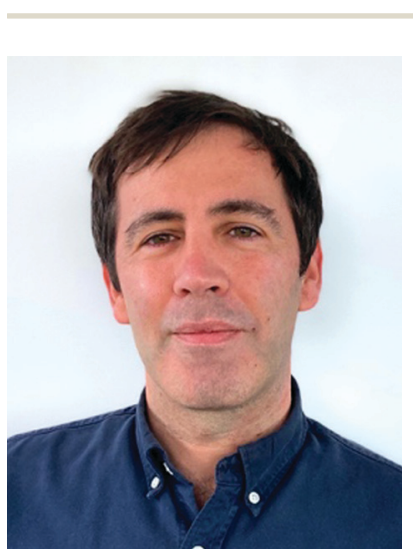

Rafael T. M de Rosales
Rafael T. M de Rosales obtained his BSc in Chemistry from the University of Granada (Spain), and $a \mathrm{PhD}$ in Bioinorganic Chemistry at the University of Edinburgh (UK) in 2004. After a Marie Curie Postdoctoral Fellowship in Naples (Italy), and a postdoctoral research position in bio-inspired inorganic catalysis at Imperial College London (UK), he moved to the School of Biomedical Engineering \& Medical Imaging at King's College London in 2007, where he is now Reader in Imaging Chemistry. His main interest is the development and application of radiochemical tools to investigate the in vivo behaviour of drug delivery systems and cell therapies.
Since the approval in 1989 of Diprivan (a liposomal-based formulation used as anaesthetic) by the Food and Drug Agency (FDA), the number of clinically-approved nanomedicines has grown remarkably. ${ }^{2}$ One of the most notable early examples is the cancer nanomedicine Doxil/Caelyx (PEGylated liposomal doxorubicin), approved in 1995 and still widely used today in ovarian cancer, HIV-associated Kaposi's sarcoma and multiple myeloma. ${ }^{3}$ Several nanomedicines have since been approved by the FDA and/or the European Medicines Agency (EMA) for different purposes such as cancer therapy, iron-replacement, vaccines, anaesthetics, fungal treatments, muscular degeneration, or imaging. ${ }^{4}$ In 2015, PEGylated liposomal irinotecan (Onivyde MM-398) was approved for metastatic pancreatic cancer. ${ }^{5}$ Moreover, liposome technology has been applied to improve vaccines (Epaxal, Inflexal V), treatments for macular degeneration (Visudyne) and fungal infections (AmBisome), among other applications. ${ }^{6,7}$ Besides liposomes, several iron oxide NP formulations are being utilised as treatment for iron deficient anaemia (Venofer, Ferrlixit, Ferinject, Feraheme). ${ }^{8}$ Although the benefits of nanomedicinal formulations are well reported - with many preclinical examples supporting their effectiveness - their translation into the clinics is still an arduous, lengthy and costly pathway with multiple issues to be addressed. ${ }^{9}$ This is clearly evidenced by the relatively few examples of pre-clinical research that have translated into clinical applications.

In preclinical research, the use of NPs is still being widely explored for both imaging and therapeutic applications. Different imaging agents based on NPs can be found for several medical imaging techniques; providing anatomical and functional information with increased sensitivity and specificity. ${ }^{10}$ From the use of NPs to simply generate contrast in imaging techniques, work in this area has evolved towards more sophisticated formulations ("smart" NPs) capable of responding to external stimuli, biological targets or microenvironmental conditions in a specific manner relevant to the diagnostic and/or treatment of a disease. ${ }^{11}$

Current medical non-invasive imaging techniques include computed tomography (CT), magnetic resonance imaging (MRI), 
optical imaging techniques (OI) and nuclear imaging techniques such as single photon emission computed tomography (SPECT) and positron emission tomography (PET). Each technique has advantages and drawbacks (see Section 2); and the choice of which imaging method is most appropriate must be carefully considered based on the clinical problem being addressed. In particular, radionuclide imaging techniques offer high sensitivity (defined as the concentration of tracer needed for contrast) and the ability to provide functional/metabolic information at the molecular level. These techniques require the use of exogenous compounds containing radioisotopes (radiotracers), to provide imaging contrast. Radiotracers usually consist of biologically active organic molecules previously modified (radiolabelled) with a SPECT or PET radionuclide (see Section 3). For instance, one of the most clinically used radiotracers for PET is ${ }^{18} \mathrm{~F}$-fluorodeoxyglucose ( $\left.\left[{ }^{18} \mathrm{~F}\right]-\mathrm{FDG}\right)$ formed by a deoxyglucose molecule radiolabelled with the radionuclide fluorine-18 $\left({ }^{18} \mathrm{~F}\right)$. Considering the role of deoxyglucose in metabolic glycolytic pathways, many clinical studies are conducted daily to detect the increased level of glycolysis found in patients with cancer and other diseases. ${ }^{12}$ Besides small molecules, nanomaterials are also being explored as radiotracers that combine the size-dependent properties of nanomaterials with the high sensitivity provided by radionuclides. Although radiolabelled nanomaterials are not applied routinely in clinics, they could find applications thanks to specific properties such as the ability to incorporate multiple radionuclides per NP (leading to high sensitivity), vector ligands (leading to high target affinity), or therapeutic components in a single platform. ${ }^{13}$ This concept, known as multifunctionality, has generated new possibilities in the application of radiolabelled nanomaterials, not only for standard or multimodal molecular imaging but also for combined diagnosis and therapy - known as 'theranostics'.

The term theranostics was introduced in 1998 by J. Funkhouser referring to "the ability to affect therapy or treatment of a disease state". ${ }^{14}$ Being able to perform therapy and diagnosis with the same vector is an important step forward towards personalised medicine where the safety and effectiveness of a treatment can be predicted and monitored by medical imaging techniques. With a slow evolution during the first years, the use of nanomedicines as theranostics platforms - known as nanotheranostics - has arguably had a large impact on the field. Different nanoparticle-based treatments such as those based on chemotherapy, gene therapy, immunotherapy, radiotherapy, photothermal therapy or photodynamic therapy have been developed in combination with the imaging modalities mentioned above. ${ }^{15-17}$ The ability to image nanoparticlebased therapeutics non-invasively can provide information on target uptake of the nanomedicines - as well as potentially predict the therapeutic response. Hence, nanotheranostic platforms can potentially guide treatment regimens on a patient-to-patient basis. Additionally, the combination of nuclear imaging modalities with radiotherapies is especially attractive. ${ }^{18}$

One of the key aspects to consider when radiolabelling nanomaterials is the selection of the radionuclide. Different properties such as half-life, decay mode and biological response must be considered in advance (see Section 3). The chemistries available to integrate the radionuclide into the nanomaterial must be then considered; with special attention given to the type of material and their potential effects on their physicochemical properties, as well as the expected in vivo stabilities. (see Section 4). These considerations are essential to avoid time-consuming and inefficient protocols that could give misleading or unusable results. The interaction between the radionuclide and the nanomaterial, the level of loading/chemical modifications and the stability of the final formulation in physiological media are key properties that will influence the pharmacokinetics and pharmacodynamics of the radiolabelled nanomaterial.

The strategies used during early nanoparticulate radiolabelling studies were primarily based on the application of standard radiochemistry protocols for lower-molecular weight compounds. With the evolution of the field, novel advanced radiolabelling methods specifically designed for the radiolabelling of nanomaterials are continuously emerging. Whether a radiolabelling method is adequate or not is affected by multiple factors that need to be carefully addressed. This review aims to discuss all these factors and provide a thorough summary and critical review of the different strategies available to label nanomaterials with radionuclides, from traditional to recent innovative methods. Ultimately, we hope that this document will guide the reader to select the best strategy for developing efficiently radiolabelled nanomaterials for innovative imaging and/or therapeutic purposes.

\section{Medical imaging techniques: focus on nuclear imaging and radionuclide therapy}

\subsection{Medical imaging}

Medical imaging refers to the use of imaging scanners to noninvasively obtain in vivo information of living subjects - as opposed to ex vivo invasive medical procedures (e.g. biopsy). Patients/subjects are placed within a medical imaging scanner which provides information, based on image contrast achieved by an intrinsic mechanism of the imaging technique (US, MRI, CT). Alternatively, image contrast can be attenuated/boosted by exogenous 'contrast agents'; which require pre- and postcontrast imaging allowing signal quantification (US, MRI, CT). Finally, imaging agents which have an inherent signal can be administered for 'hot-spot' imaging (e.g. ${ }^{19} \mathrm{~F}-\mathrm{MRI}$, radioactive agents and fluorescent dyes). Depending on the technique, anatomical information and/or data on real-time biochemical processes (i.e. molecular imaging) ${ }^{19}$ can be obtained. The medical imaging modalities available have important differences in their properties (Table 1), including: imaging field of view (FOV), spatial and temporal resolution, sensitivity, and tissue depth limitation of the imaging signal. Multimodal imaging, in which two or more imaging modalities are combined into a single instrument, is often used to overcome some of the drawbacks associated with any imaging technique by providing synergistic information. In this review we focus on radionuclidebased imaging methods, however, to gain a good understanding of 
Table 1 Summary of the properties of the imaging modalities used for nanoparticle imaging discussed in this review. $\mathrm{PC}=$ preclinical scanner; $\mathrm{C}=$ clinical scanner. Adapted from ref. 19-22

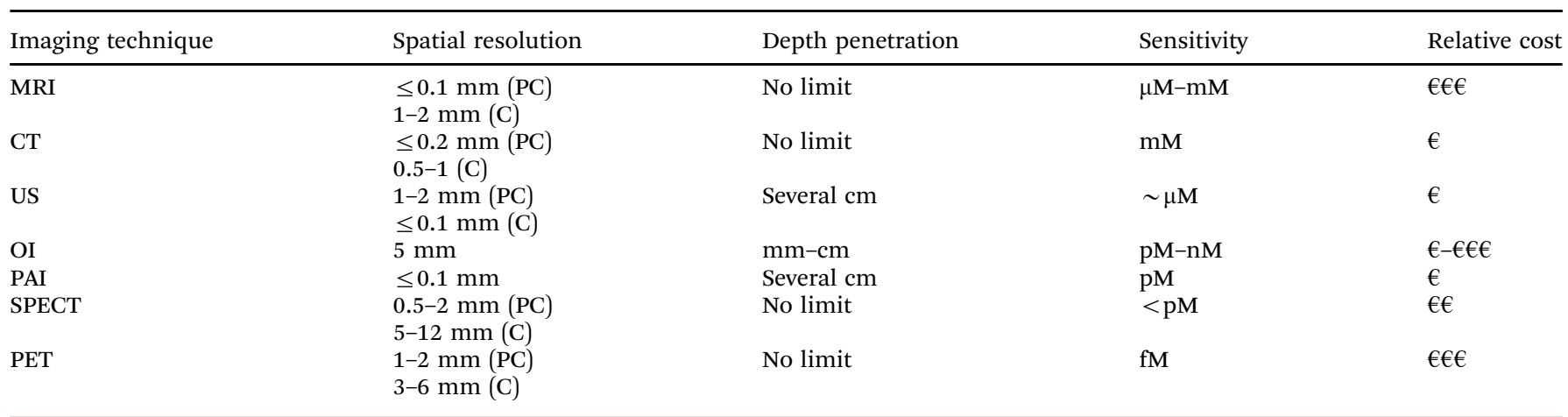

the pros and cons of these techniques for imaging NPs, we will provide a brief overview of other non-radionuclide based imaging modalities.

2.1.1 Magnetic resonance imaging (MRI). Magnetic resonance imaging (MRI) relies on the spin characteristics and magnetic properties of certain atomic nuclei. The primary nuclei used for MRI contrast are protons $\left({ }^{1} \mathrm{H}\right)$ present abundantly in water molecules within the body. Protons in different tissue environments (e.g. fatty tissue or blood) have different relaxation times, which allow image contrast. ${ }^{19}$ The imaging contrast in MRI is generated due to the different longitudinal $\left(T_{1}\right)$ and transverse $\left(T_{2}\right)$ relaxation times of each tissue. NPs containing paramagnetic metals $\left(\right.$ e.g. $\mathrm{Gd}^{3+}$ and $\left.\mathrm{Mn}^{2+/ 3+}\right)$ are capable of modulating the relaxation times of MRI-active nuclei. For example, Gd-NPs can provide $T_{1}$-weighted (positive) contrast allowing imaging (Fig. 2). ${ }^{23}$ Superparamagnetic iron oxide nanoparticles (SPIONs) provide contrast mainly by $T_{2}$-weighted (negative) protocols, ${ }^{24}$ but can also provide $T_{1}$-based contrast depending on their properties (Fig. 2). ${ }^{25}$ As well as imaging ${ }^{1} \mathrm{H}$, other nuclei such as ${ }^{19} \mathrm{~F}$ can be detected with MRI after exogenous administration of fluorine-containing NPs (Fig. 2) allowing 'hot-spot' MR imaging. MRI as a modality provides exceptional spatial resolution (for ${ }^{1} \mathrm{H}$-MRI: $c a .0 .1 \mathrm{~mm}$ pre-clinically; $c a .1 \mathrm{~mm}$ clinically) and benefits from not requiring ionising radiation. However, it has limited applications in molecular imaging due its low sensitivity $\left(10^{-3}-10^{-5} \mathrm{M}\right)$ and the difficulties of performing whole-body MRI and obtaining quantitative images.

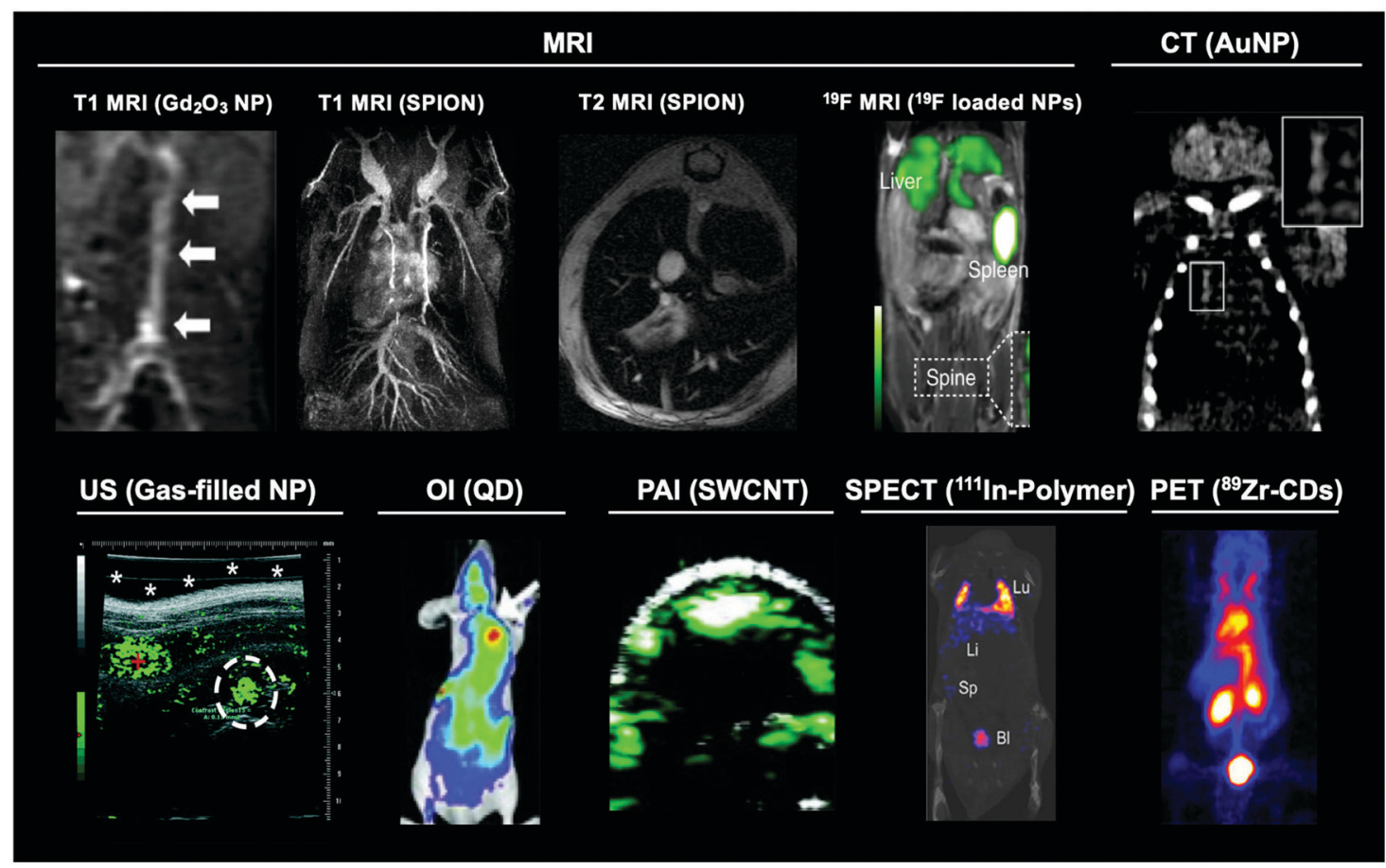

Fig. 2 Representative images of the main modalities used to image different nanomaterials. $\mathrm{Gd}_{2} \mathrm{O}_{3}$ nanoparticle MR image adapted from Park et al. ${ }^{26}$ $T_{1}$ and $T_{2}$ SPION MRI image adapted from Pellico et al. ${ }^{27}{ }^{19} \mathrm{~F} \mathrm{MRI} \mathrm{image} \mathrm{adapted} \mathrm{from} \mathrm{Senders} \mathrm{et} \mathrm{al.}{ }^{28} \mathrm{CT}$ image adapted from Chhour et al. ${ }^{29} \mathrm{US}$ image adapted from Peyman et al. ${ }^{30} \mathrm{Ol}$ image adapted from Gao et al. ${ }^{31}$ PAl image adapted with permission from de la Zerda et al. ${ }^{32}$ Copyright (2010) American Chemical Society. SPECT image adapted from Imlimthan et al. ${ }^{33}$ PET image adapted from Cheng et al. ${ }^{34}$ 
2.1.2 Computed tomography (CT). Computed tomography (CT) is a widely available medical imaging technique based on the differing levels of X-ray attenuation in the body. Based on their density and composition, tissues will either strongly absorb (e.g. bone) or weakly absorb (e.g. air) X-rays resulting in imaging signal contrast. CT provides 3D images at high spatial resolution ( $c a$. $0.1 \mathrm{~mm}$ pre-clinically and $c a .0 .5 \mathrm{~mm}$ clinically) and has no imaging signal depth limitation. However, the use of highly ionising X-rays results in high radiation doses. ${ }^{19}$ Whilst primarily used for anatomical information, NPs containing high concentrations of high $Z$ elements can be used as CT contrast agents (e.g. Au, I, Yb, Ba) resulting in high spatial-resolution in vivo images (Fig. 2). ${ }^{35-37}$ The low sensitivity of this technique, however, results in the need of high concentrations for in vivo detection that could lead to potential toxicity issues and limitations for molecular imaging.

2.1.3 Ultrasound (US) imaging. Ultrasound (US) imaging relies on the properties of high-frequency sound waves as they travel through tissues. During a US scan, a transducer is externally placed on the target area where it emits pulses of high frequency sound waves. These sound waves enter the body and are reflected back (backscattered) where they are detected by the transducer again. The properties of the reflected soundwaves, such as their frequency, amplitude and time of arrival, are analysed and allow a $2 \mathrm{D}$ image to be created..$^{38}$ Ultrasound imaging is low-cost, does not use ionising radiation, provides excellent spatiotemporal resolution (essentially providing real-time imaging), and is widely used in the clinical setting. Despite this, it has a very small field of view (it cannot be performed on a whole-body scale) and suffers from limited tissue depth penetration. Particulate materials such as microbubbles or nanobubbles that scatter US waves can be imaged with this imaging technique (Fig. 2), ${ }^{23}$ a property that is often used to enhance US images and allow diagnoses in the clinical setting. ${ }^{39}$

2.1.4 Optical imaging (OI). Optical imaging (OI) is based on the detection of light emissions from molecules after their excitation. These light emissions and their intensity are detected by external cameras that convert this information into images. For in vivo applications, optical fluorescence imaging is often used and relies on exogenous chemical compounds as imaging agents that fluoresce after excitation from an external light source of a certain wavelength. Any NP with fluorescent emission properties (e.g. quantum dots) can thus be imaged using this technique, with the advantage that they can be imaged at multiple spatial scales, from whole body (Fig. 2) to the cell level (microscopy) However, OI suffers from limited tissue depth limitations both for the excitation and emission lights, as well as significant tissue autofluorescence, that limit its in vivo imaging applications to the intraoperative and preclinical fields.

2.1.5 Photoacoustic imaging (PAI). Photoacoustic (or optoacoustic) imaging (PAI) is based on the detection of acoustic waves, which are generated by endogenous chromophores and/or administered contrast agents - following their absorption of light pulses (Fig. 2). ${ }^{40}$ PAI is highly sensitive and has a comparably high spatial resolution to US imaging (Table 1). Although, it also suffers from a limited FOV and tissue penetration limits. Despite this, due to the lower scattering of sound waves by tissue, compared with light photons, PAI has a higher depth penetration compared with standard OI techniques. ${ }^{22}$ Furthermore, multispectral PAI allows images generated to be spectrally unmixed, thus allowing imaging of multiple chromophores. ${ }^{40}$ A variety of nanomaterials can be used as contrast agents for PAI; including gold NPs, carbon nanomaterials and - more recently - semi-conducting polymer nanoparticles. ${ }^{2,41}$

\subsection{Radionuclide imaging}

Radionuclide or nuclear imaging refers to two main imaging techniques: single-photon emission computed tomography (SPECT, Fig. 3A) or positron emission tomography (PET, Fig. 3B). Both of these techniques rely on the detection of radioactive nuclides (radionuclides). Thus, tracking NPs using PET/SPECT requires their 'tagging' or 'labelling' with radionuclides (radiolabelling) allowing non-invasive in vivo imaging via the radioactive decay emissions of the radionuclide - using the appropriate scanner. Both techniques, however, differ in the detection method, leading to significant differences that are worth discussing below.

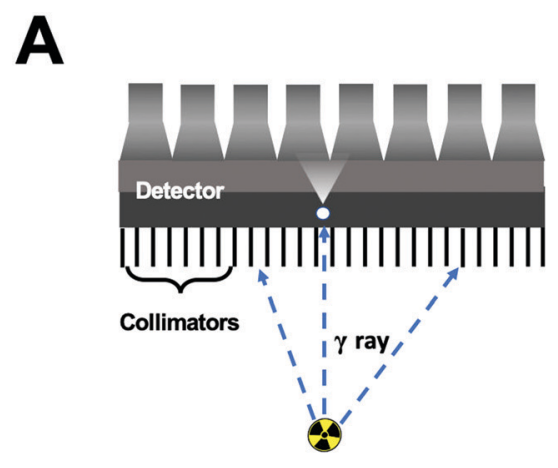

Single photon emission computed tomography (SPECT)

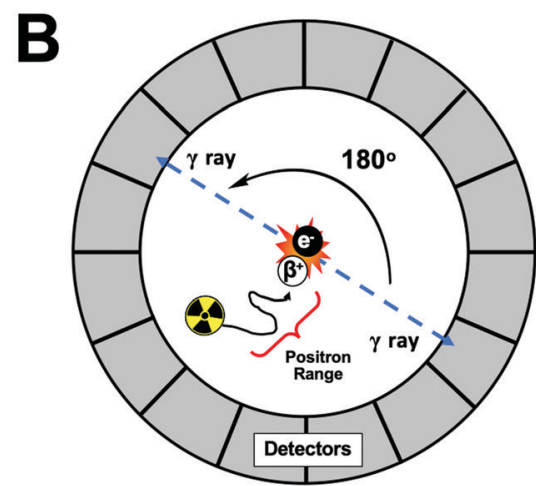

Positron emission tomography (SPECT)

Fig. 3 (A) Schematic representation of single photon emission computed tomography (SPECT), (B) schematic representation of positron emission tomography (PET). 
2.2.1 Single photon emission computed tomography (SPECT). Single photon emission computed tomography (SPECT) imaging uses radionuclides that emit gamma ray photons during their radioactive decay (vide infra, Section 3.2). The emitted gamma rays have defined energy levels which are detected using a gamma camera. SPECT is performed by rotating the camera around the subject or patient to capture the gamma emissions in $3 \mathrm{D}$. To determine the origin of the photons, collimators that preferentially allow parallel rays are used (Fig. 3A). Hence, narrow collimators (e.g. multi-pinhole) allow high spatial resolution SPECT imaging. However, this is achieved at the expense of sensitivity since the process of collimation excludes a significant amount of diagonally incident photons. The balance between collimator aperture and associated spatial resolution often determines the amount of radioactivity and scanning time required for different SPECT imaging applications.

2.2.2 Positron emission tomography (PET). Positron emission tomography (PET) involves the imaging of radionuclides that decay by emitting positrons $\left(\beta^{+}\right)$, which are the anti-matter equivalent of electrons (vide infra, Section 3.3). Once the released positrons interact with nearby electrons they undergo annihilation, releasing energy in the form of two gamma ray photons emitted in opposite directions and angle to each other (ca. $180^{\circ}$ ) and a distinct energy of $511 \mathrm{keV}$ (Fig. 3B). PET cameras are made up of a ring of detectors for the detection of these $511 \mathrm{keV}$ gamma rays (known as coincidence detection). The precise origin of the annihilation event along a so-called 'line of response' - and therefore the approximate location of the PET radionuclide - can then be determined with a spatial resolution in the $\mathrm{mm}$ range, as determined by the positron range/energy of each radionuclide (Fig. 3B).

2.2.3 PET vs. SPECT imaging. Now that we have briefly discussed the basic concepts behind both nuclear imaging techniques, we will outline how these differences influence their individual capabilities. In terms of spatial resolution, we described above how the use of collimators in SPECT allows the potential for high spatial resolution. ${ }^{19}$ However, that of clinical SPECT scanners $(5-12 \mathrm{~mm})$ is lower than with clinical PET scanners (3-6 mm). This is largely the result of the balance discussed above that is required between collimator aperture and radioactivity dose. However, in the preclinical setting, differences in resolution between the two modalities $(c a .1 \mathrm{~mm})$ are minor. ${ }^{42}$ The sensitivity of PET is superior to that of SPECT due to the lack of collimation in the former, which also results in improved signal quantification. Despite this, clinical SPECT imaging is less costly and more widely available. Additionally, due to the unique energy emissions that SPECT radionuclides have, multiple isotopes and radioactive compounds can be imaged independently within the same in vivo imaging subject - known as multiplexed imaging. ${ }^{21}$ In contrast, all annihilation event gamma rays emitted by PET isotopes have the same $511 \mathrm{keV}$ energy, making multiplexed imaging of multiple compounds not currently possible with standard scanners. However, many PET radionuclides also produce additional gamma emissions, which can lead to triplecoincidence events. These can be detected with additional gammaray detectors allowing the detection of multiple PET isotopes within the same system. ${ }^{43}$ Despite its lower global availability, there are an increasing number of PET scanners and radiotracers becoming available in clinics worldwide, due to the superior sensitivity and spatial resolution. Finally, the recent breakthrough in the PET imaging field of the clinical total-body scanner technology should be highlighted. Using total-body PET imaging radiotracers can be imaged in humans at much lower radiation doses (up to $40 \times$ lower), and significantly lower acquisition times. ${ }^{44,45}$

2.2.4 Advantages and disadvantages of PET and SPECT for nanoparticle imaging (vs. other medical imaging techniques). Both nuclear imaging techniques have key properties that make them highly suited to image the biodistribution and pharmacokinetics of NPs in vivo. First is the issue of imaging signal tissue penetration. PET and SPECT have no tissue depth penetration limits, as the high-energy gamma-ray photons emitted by radionuclides can easily pass through tissue, and can be performed on a whole body scale. Additionally, they are greatly more sensitive $\left(10^{-10}-10^{-12} \mathrm{M}\right)$ compared to other imaging modalities such as MRI and CT. These properties combined mean that clinical and preclinical imaging can be performed using small quantities of NP radiotracer; in the order of micrograms or lower - compared with milligram to gram quantities of NPs for MRI/CT. A key benefit is that this low amount of NP radiotracer required does not perturb the biological system of interest, and is less likely to induce toxic effects. Furthermore, the use of radionuclides allows the accurate quantification of NP tissue uptake in vivo with high temporal resolution, as well as ex vivo. This is particularly important and challenging to achieve with MRI/CT and allows the use of nuclear imaging techniques for whole-body analysis of NP pharmacokinetics and biodistribution. Despite these properties, nuclear imaging offers lower spatial resolution compared with MRI and CT. To overcome this, nuclear imaging techniques are often combined with CT, or more recently MRI, to provide synergistic high spatial resolution anatomical information. An additional important consideration when using radionuclides is the radiation doses each subject receives during scanning, which must be considered and are often minimal when carefully managed.

\subsection{Radionuclide therapy}

The decay properties of certain radionuclides allow their use as therapeutics, adding the possibility of using NPs as radionuclide therapy agents. These radionuclides emit $\alpha$ (alpha), $\beta^{-}$ (beta) particles or Auger electrons that are capable of depositing a substantial amount of energy, and hence damage, to tissues. These therapeutic radionuclides can be incorporated in high concentrations into nanomaterials with the aim of delivering their radio-emission 'payload' to specific tissues (e.g. tumours). ${ }^{46,47}$ For maximum therapeutic efficacy, the radionuclide decay type, range, and the energy deposited over that distance - the linear energy transfer (LET) - must be carefully considered and matched to the biological target. ${ }^{48}$ The three emission types for radionuclide therapy will be briefly summarised below.

2.3.1 Alpha-particle radiation. An alpha particle is a helium $\left({ }^{4} \mathrm{He}\right)$ nucleus, with a +2 -charge emitted, by certain radionuclides 
A

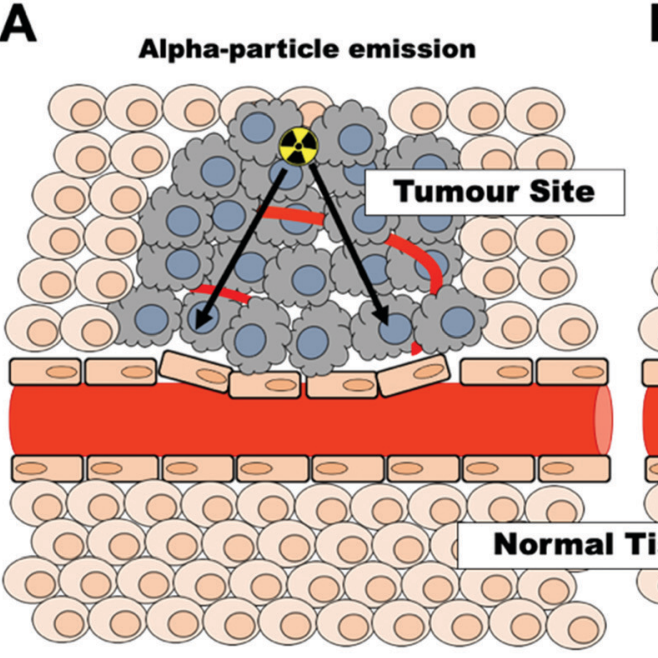

C

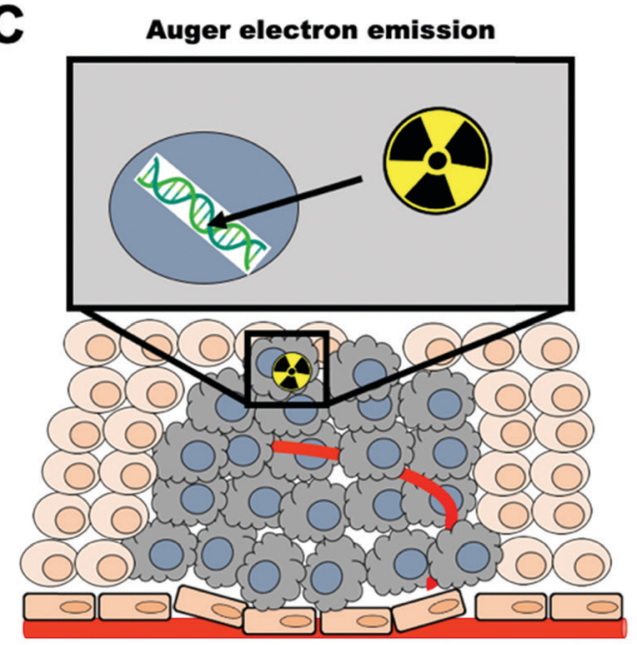

B

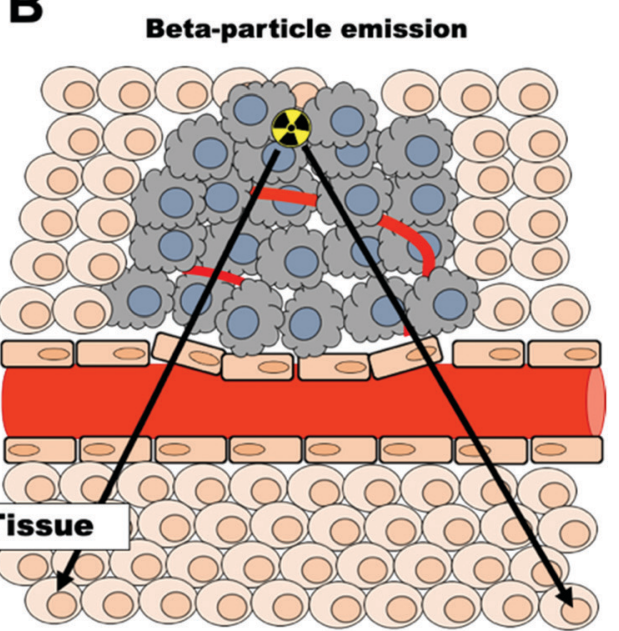

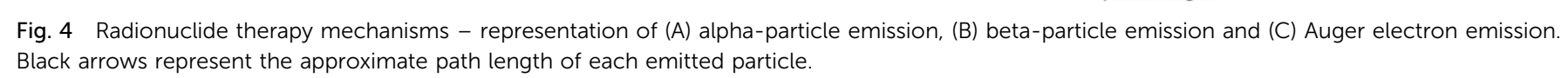

as they undergo radioactive decay. Alpha particles are considered to have a high linear energy transfer (LET) of approximately $80 \mathrm{keV} \mu \mathrm{m}^{-1}, 48$ and a particle range of $50-100 \mu \mathrm{m}$, and hence can deposit energy over $c a$. 5-10 cell diameters (Fig. 4A) ${ }^{49}$ The primary molecular target of alpha-particle radiotherapy is the DNA within the cell nucleus, causing double-strand breaks, but cytotoxicity is likely to involve a number of other mechanisms such as reactive oxygen species (ROS) generation. ${ }^{50}$ Additionally, due its particle range and LET, alpha particles are capable of damaging neighbouring cells - known as the cross-fire effect. ${ }^{48}$ Examples of common alpha-emitting radionuclides can be found in Table 4.

2.3.2 Beta-particle radiation. A beta particle $\left(\beta^{-}\right)$is a high energy electron emitted from a decaying radionuclide. These should not be confused with positrons $\left(\beta^{+}\right)$which are another type of beta particle. $\beta^{-}$particles have a low LET $\left(0.1-1.0 \mathrm{keV}_{\mu \mathrm{m}}^{-1}\right)$, resulting in the largest particle range $(\leq 12 \mathrm{~mm})$, relating to many hundreds of cell diameters compared with alpha particles and Auger electrons. ${ }^{48,49}$ This can result in the damaging of healthy tissue surrounding tumour sites (Fig. 4B) via the cross-fire effect.
2.3.3 Auger electron radiation. Auger electrons are electrons ejected from radioactive nuclei due to the Auger effect. During a radioactive decay a vacancy in an inner electron orbital can occur, which is then filled by an outer electron shell. The energy difference from this transition is then transferred to another electron where it is finally ejected from the atom. This ejected electron is known as an Auger electron. Auger electrons have a very small particle range $(<0.5 \mathrm{~mm})$, but with a high LET (1-26 keV $\left.\mu \mathrm{m}^{-1}\right)^{49,51}$ and so ideally have to be delivered intracellularly to the nucleus to maximise the cytotoxic activity from DNA double stand breaks (Fig. 4C). Despite this, Auger electrons can also induce cell death by damaging the cell membrane, as well as via ROS generation..$^{51}$ Further details on Auger-emitting radionuclides can be found in Section 3.4 and Table 4.

\section{Radionuclides}

\subsection{Production of radionuclides}

Traditionally, the production of radionuclides for medical imaging and therapy has been associated with costly facilities 


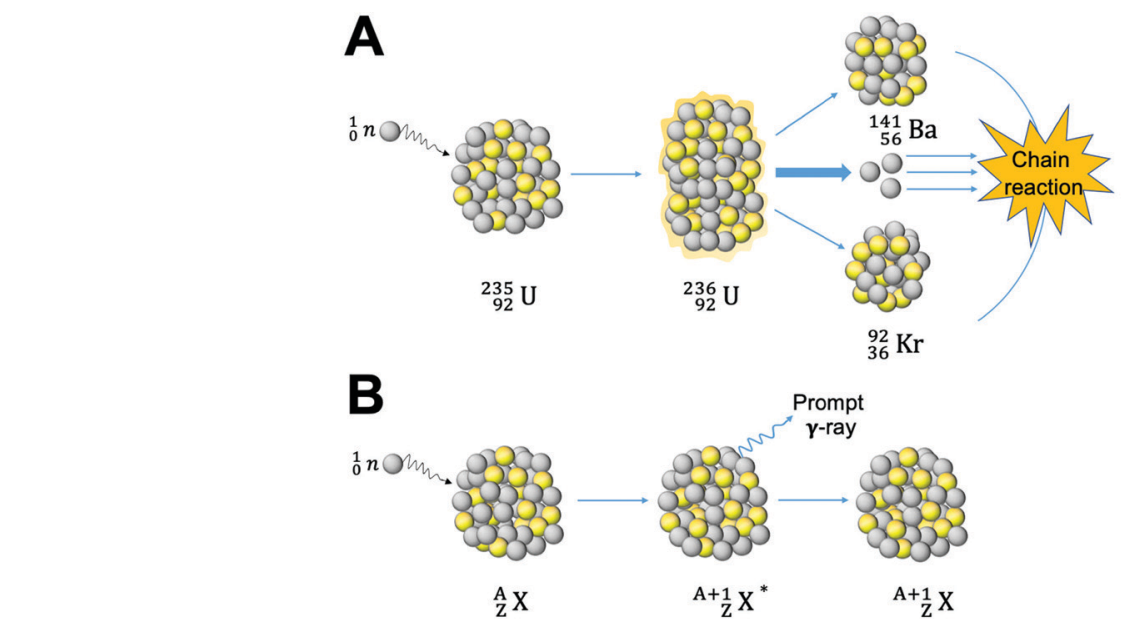

C

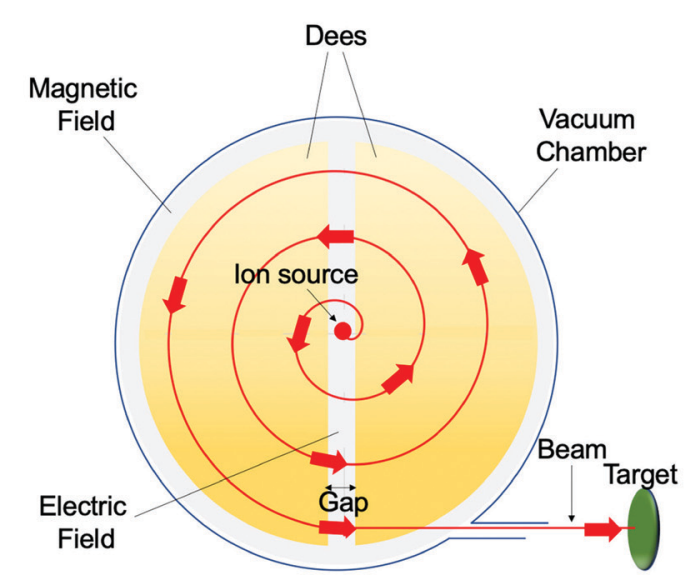

D Radionuclide generator

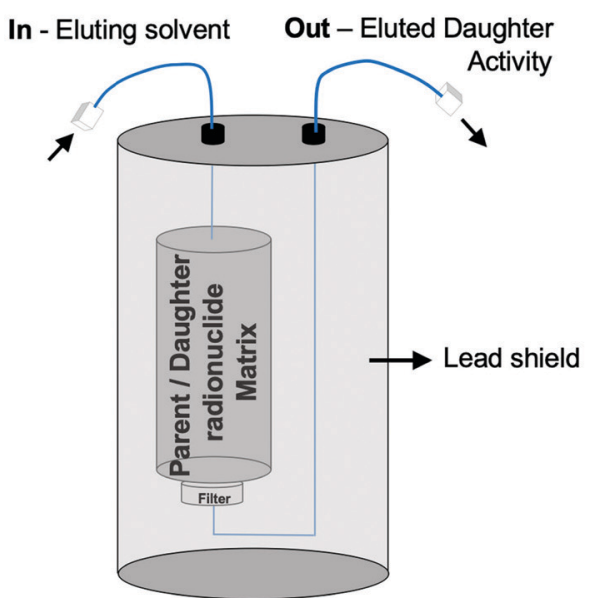

Fig. 5 Production of radionuclides. Schematic representation of (A) nuclear fission of a ${ }^{235} \mathrm{U}$ atom, (B) a $(\mathrm{n}, \gamma)$ neutron activation process, (C) cyclotron, and (D) standard radionuclide generator.

and time-consuming protocols. Nevertheless, the optimisation of production processes and the modernisation of production technologies has facilitated their increased use in the clinical and preclinical settings. Four methods are currently applied for radionuclide production: fission, neutron activation, cyclotron and generator. These will be briefly described below.

3.1.1 Fission and neutron activation. Both fission and neutron activation methods are triggered by the bombardment of a stable nuclide (target) with a neutron, and require energies only available at nuclear reactors. In fission, the neutron penetrates into the nucleus of the target generating a highly unstable nuclide that consequently undergoes nuclear fission generating a new pair of atoms, $\gamma$-ray emissions and two to three neutrons (Fig. 5A). ${ }^{52}$ One of the most important radionuclides applied in nuclear medicine and produced by fission is ${ }^{99} \mathrm{Mo}$ with a major application as the parent radionuclide in ${ }^{99} \mathrm{Mo} /{ }^{99 \mathrm{~m}} \mathrm{Tc}$ generators (vide infra).

Neutron activation is the other process carried out in a nuclear reactor. Here, the neutrons generated during the fission reaction are directed to a target with a stable nuclide, $\mathrm{z}^{\mathrm{A}} \mathrm{X}$, giving an excited product nucleus, $\mathrm{z}^{\mathrm{A}+1} \mathrm{X}^{*}$. This excited nucleus then undergoes de-excitation to a ground state emitting a prompt $\gamma$ photon, yielding a radioactive isotope of the same element, $\mathrm{z}^{\mathrm{A}+1} \mathrm{X}$ (Fig. 5B). Although the $(\mathrm{n}, \gamma)$ reaction is the most common in neutron activation, $(\mathrm{n}, \mathrm{p})$ reactions can also occur by emission of a proton, $p$. In this case, the starting target and the obtained product are different elements with the reaction represented as ${ }_{\mathrm{Z}}^{\mathrm{A}} \mathrm{X}(\mathrm{n}, \mathrm{p})_{\mathrm{Z}-1}{ }^{\mathrm{A}} \mathrm{Y}$.

3.1.2 Cyclotron. A cyclotron is a particle accelerator where particles (protons, deuterons, Triton or $\alpha$-particles) generated by an ion source at high voltage, are accelerated following a spiral trajectory and directed towards a target (Fig. 5C). To accelerate the particles, two semi-circular electrodes (Dees or "Ds") are placed between the poles of an electromagnet under vacuum separated by a narrow gap. The change of polarity between the electromagnet poles allow the particles to cross the gap travelling from one $\mathrm{D}$ to the other while increasing the speed. ${ }^{53}$ Contrary to nuclear reactors, where 
nuclides often decay by $\beta^{-}$due to the overabundance of neutrons, the cyclotron-produced radionuclides are deficient in neutrons and decay by EC or $\beta^{+}$. Therefore, cyclotrons are the main production method for positron emitting radionuclides.

3.1.3 Generator. A generator is a piece of benchtop equipment containing a solid matrix where a pair of parent/daughter radionuclides are adsorbed. The concept is based on the selective extraction of the daughter radionuclide from the matrix via a solvent elution method (Fig. 5D). This separation is based on either physical or chemical properties of the two radionuclides. Moreover, due to the higher half-life of the parent radionuclide, the generator might be eluted repeatedly (usually a recovery time is required) allowing a continuous supply of the daughter activity. Generators have other unique advantages such as a small footprints and simple set up and use, avoiding costly bespoke facilities. ${ }^{54}$ In addition, generators provide "on site" radionuclides with very short half-life times such as ${ }^{82} \mathrm{Rb}\left(t_{1 / 2}=76 \mathrm{~s}\right)$ or ${ }^{62} \mathrm{Cu}\left(t_{1 / 2}=9.7 \mathrm{~min}\right)$. However, only a few parent/daughter pairs are amenable for routine generator production at the preclinical and clinical settings (Tables 2-4).

\subsection{Radionuclides for SPECT}

Radionuclides are mainly characterised by their decay modes, the energy emitted and the half-life of the products and subproducts generated until the stable isotope is reached. ${ }^{5,56}$ Gamma-emitters have been used since the beginning of nuclear medicine for $\gamma$-scintigraphy. With the development of SPECT - usually combined with $\mathrm{CT}$ - $\gamma$-emitting radionuclides are expanding the clinical imaging applications beyond the traditional $\gamma$-cameras. Nowadays, ${ }^{99 m}$ Tc is the most widely used radionuclide. This radionuclide combines a moderate short half-life $(6 \mathrm{~h})$, appropriate nuclear properties ( $89 \%$ of $\gamma$-rays abundance at $140 \mathrm{keV}$ ) and accessible generator production; making it a highly suitable choice for nuclear imaging studies. ${ }^{57}$ Due to its metallic character and several oxidation states available, radiolabelling with ${ }^{99} \mathrm{~m}$ Tc is based on the formation of coordination complexes between the radionuclide (that needs to be reduced from Tc(vII) and a chelating ligand). Therefore, the versatility of ${ }^{99 \mathrm{~m}}$ Tc based radiolabelling, and that of other metallic radionuclides, is limited to coordination chemistry approaches (see Section 4.2). ${ }^{58}$ Other SPECT radionuclides, mainly iodine isotopes, are used for the formation of covalent bonds with carbon. In this regard, iodine radionuclides offer different isotopes to perform medium-term $\left({ }^{123} \mathrm{I}, t_{1 / 2}=\right.$ $13.3 \mathrm{~h}$ ) or long-term imaging studies $\left({ }^{125} \mathrm{I}, t_{1 / 2}=60.5 \mathrm{~d}\right)$ and even radiotherapy $\left({ }^{131} \mathrm{I}, t_{1 / 2}=8 \mathrm{~d}, \beta^{-}\right)$with the same molecule. ${ }^{59}$ There is an extensive variety of useful SPECT radionuclides; not only for the radiolabelling of small molecules, peptides, proteins or antibodies, but also for the radiolabelling of nanomaterials (Table 2).

\subsection{Radionuclides for PET}

Traditionally, clinical applications of PET have been mainly focused on four radionuclides: ${ }^{11} \mathrm{C},{ }^{18} \mathrm{~F},{ }^{13} \mathrm{~N}$ and ${ }^{15} \mathrm{O} .{ }^{60}{ }^{18} \mathrm{~F}$ is currently the main radionuclide used in clinical PET imaging,

Table 2 Radionuclides for SPECT imaging discussed in this review

\begin{tabular}{|c|c|c|c|c|c|}
\hline Radionuclide & Half-life & Max. energy (keV) & Decay & Production & Common production reaction \\
\hline Au-198 & $2.7 \mathrm{~d}$ & 960 & $\beta^{-}, \gamma$ & Cyclotron & ${ }^{197} \mathrm{Au}(\mathrm{n}, \gamma){ }^{198} \mathrm{Au}$ \\
\hline Au-199 & $3.1 \mathrm{~d}$ & 452.6 & $\beta^{-}, \gamma$ & Cyclotron & ${ }^{198} \mathrm{Au}(\mathrm{n}, \gamma){ }^{199} \mathrm{Au}$ \\
\hline Co-57 & $270 \mathrm{~d}$ & 692 & $\mathrm{EC}, \gamma$ & Cyclotron & ${ }^{56} \mathrm{Fe}(\mathrm{d}, \mathrm{n}){ }^{57} \mathrm{Co}$ \\
\hline Fe-59 & $44.5 \mathrm{~d}$ & 1291 & $\beta^{-}, \gamma$ & Cyclotron & ${ }^{59} \mathrm{Co}(\mathrm{p}, \mathrm{n}){ }^{59} \mathrm{Fe}$ \\
\hline Ga-67 & $78.3 \mathrm{~h}$ & 300 & Auger $\mathrm{e}^{-}, \gamma$ & Cyclotron & ${ }^{68} \mathrm{Zn}(\mathrm{p}, 2 \mathrm{n}){ }^{67} \mathrm{Ga}$ \\
\hline Gd-153 & $240.4 \mathrm{~d}$ & 103 & $\mathrm{EC}, \gamma$ & Cyclotron & ${ }^{152} \mathrm{Gd}(\mathrm{n}, \gamma){ }^{153} \mathrm{Gd}$ \\
\hline In-111 & $2.81 \mathrm{~d}$ & 245 & $\gamma$ & Cyclotron & ${ }^{111} \mathrm{Cd}(\mathrm{p}, \mathrm{n}){ }^{111} \mathrm{In}$ \\
\hline $\mathrm{I}-123$ & $13.3 \mathrm{~h}$ & 159 & Auger $\mathrm{e}^{-}, \gamma$ & Cyclotron & ${ }^{127} \mathrm{I}(\mathrm{p}, 5 \mathrm{n}){ }^{123} \mathrm{Xe}$ \\
\hline Re-186 & $91 \mathrm{~h}$ & 1080 & $\beta^{-}, \gamma$ & Cyclotron & ${ }^{186} \mathrm{~W}(\mathrm{p}, \mathrm{n}){ }^{186} \mathrm{Re}$ \\
\hline Tc-99m & $6.0 \mathrm{~h}$ & 140 & $\gamma$ & Generator & ${ }^{99} \mathrm{Mo} /{ }^{99} \mathrm{~m} \mathrm{Tc}$ \\
\hline Tl-201 & $3.0 \mathrm{~d}$ & 71 & $\gamma$ & Cyclotron & ${ }^{203} \mathrm{Tl}(\mathrm{p}, 3 \mathrm{n}){ }^{201} \mathrm{~Pb}$ \\
\hline
\end{tabular}

Table 3 Radionuclides for PET imaging discussed in this review

\begin{tabular}{|c|c|c|c|c|c|}
\hline Radionuclide & Half-life & Max. energy (keV) & Decay & Production & Common production reaction \\
\hline As-72 & $25.9 \mathrm{~h}$ & 3320 & $\beta^{+}$ & Cyclotron & ${ }^{72} \mathrm{Ge}(\mathrm{p}, \mathrm{n}){ }^{72} \mathrm{As}$ \\
\hline $\mathrm{Br}-76$ & $16 \mathrm{~h}$ & 3980 & $\beta^{+}$ & Cyclotron & ${ }^{76} \mathrm{Se}(\mathrm{p}, \mathrm{n}){ }^{76} \mathrm{Br}$ \\
\hline C-11 & $20.4 \mathrm{~min}$ & 961 & $\beta^{+}$ & Cyclotron & ${ }^{14} \mathrm{~N}(\mathrm{p}, \alpha){ }^{11} \mathrm{C}$ \\
\hline $\mathrm{Cu}-62$ & $9.7 \mathrm{~min}$ & 2926 & $\beta^{+}$ & Generator & ${ }^{62} \mathrm{Zn} /{ }^{62} \mathrm{Cu}$ \\
\hline $\mathrm{Cu}-64$ & $12.7 \mathrm{~h}$ & 656 & $\mathrm{EC}, \beta^{+}, \beta^{-}$ & Cyclotron & ${ }^{64} \mathrm{Ni}(\mathrm{p}, \mathrm{n}){ }^{64} \mathrm{Cu}$ \\
\hline $\mathrm{F}-18$ & $109.7 \mathrm{~min}$ & 634 & $\mathrm{EC}, \beta^{+}$ & Cyclotron & ${ }^{18} \mathrm{~F}\left(\mathrm{~F}^{-}\right):{ }^{18} \mathrm{O}(\mathrm{p}, \mathrm{n}){ }^{18} \mathrm{~F}$ \\
\hline Ga-68 & $67.6 \mathrm{~min}$ & 1899 & $\mathrm{EC}, \beta^{+}$ & Generator/cyclotron & ${ }^{68} \mathrm{Ge} /{ }^{68} \mathrm{Ga}$ \\
\hline Ge-69 & $39.1 \mathrm{~h}$ & 1205 & $\beta^{+}$ & Cyclotron & ${ }^{69} \mathrm{Ga}(\mathrm{p}, \mathrm{n}){ }^{69} \mathrm{Ge}$ \\
\hline $\mathrm{I}-124$ & $4.2 \mathrm{~d}$ & 2100 & $\mathrm{EC}, \beta^{+}$ & Cyclotron & ${ }^{124} \mathrm{Te}(\mathrm{p}, \mathrm{n}){ }^{124} \mathrm{I}$ \\
\hline $\mathrm{Mn}-52$ & $5.6 \mathrm{~d}$ & 1434 & $\beta^{+}$ & Cyclotron & ${ }^{52} \mathrm{Cr}(\mathrm{p}, \mathrm{n}){ }^{52} \mathrm{Mn}$ \\
\hline $\mathrm{N}-13$ & $9.9 \min$ & 1199 & $\beta^{+}$ & Cyclotron & ${ }^{16} \mathrm{O}(\mathrm{p}, \alpha){ }^{13} \mathrm{~N}$ \\
\hline $\mathrm{O}-15$ & $2.1 \mathrm{~min}$ & 1732 & $\beta^{+}$ & Cyclotron & ${ }^{15} \mathrm{~N}(\mathrm{p}, \mathrm{n})^{15} \mathrm{O}$ \\
\hline $\mathrm{Rb}-82$ & $1.3 \mathrm{~min}$ & 3378 & $\mathrm{EC}, \beta^{+}$ & Generator & ${ }^{82} \mathrm{Sr} /{ }^{82} \mathrm{Rb}$ \\
\hline Y-86 & $14.7 \mathrm{~h}$ & 3150 & $\beta^{+}$ & Cyclotron & ${ }^{86} \mathrm{Sr}(\mathrm{p}, \mathrm{n}){ }^{86} \mathrm{Y}$ \\
\hline Zr-89 & $78.4 \mathrm{~h}$ & 900 & $\mathrm{EC}, \beta^{+}$ & Cyclotron & ${ }^{89} \mathrm{Y}(\mathrm{p}, \mathrm{n}){ }^{89} \mathrm{Zr}$ \\
\hline
\end{tabular}


Table 4 Radionuclides for therapy applications

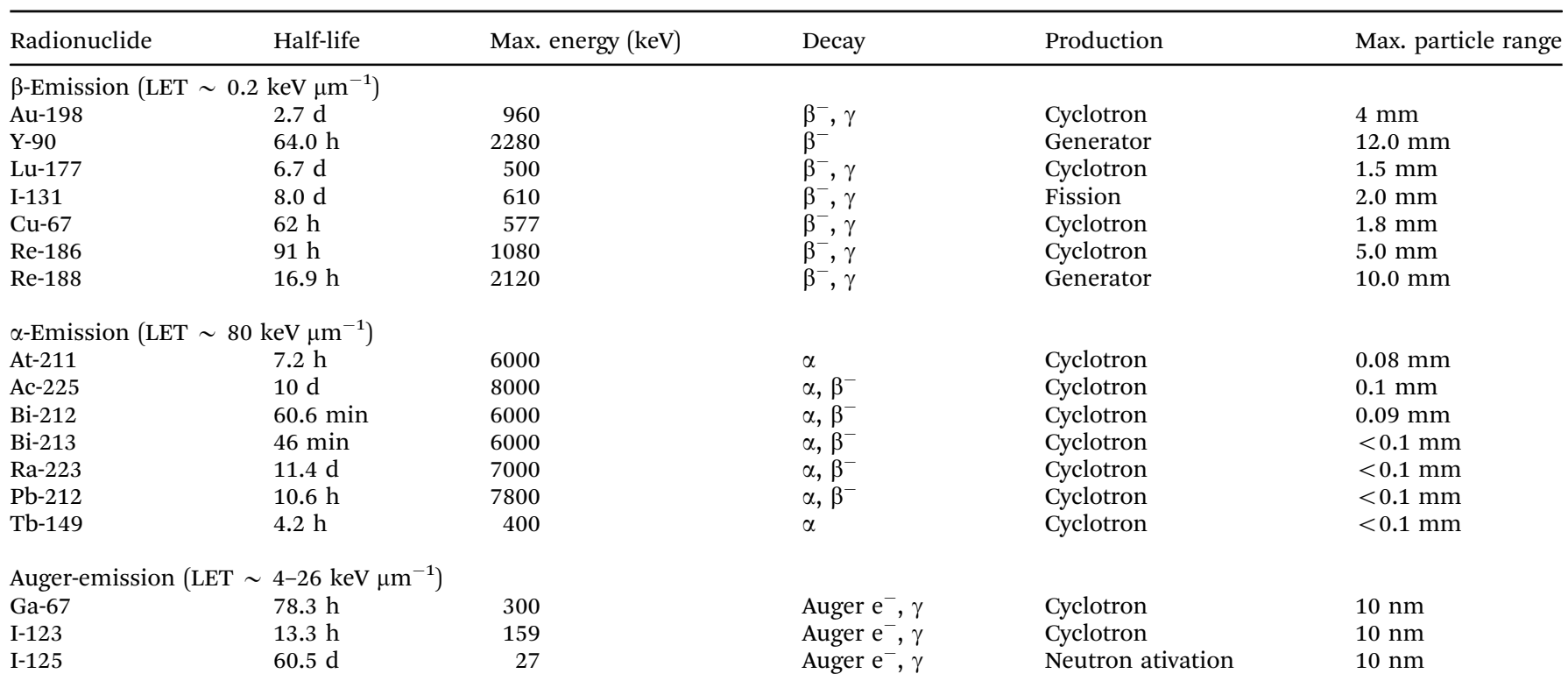

mostly due to its manageable half-life $\left(t_{1 / 2}=109.7 \mathrm{~min}\right)$, whereas that of ${ }^{11} \mathrm{C},{ }^{13} \mathrm{~N}$ and ${ }^{15} \mathrm{O}$ are very short $\left(t_{1 / 2}=\right.$ few minutes). Therefore, whereas having a cyclotron in close proximity and very fast radiolabelling protocols are required for ${ }^{11} \mathrm{C}$, ${ }^{13} \mathrm{~N}$ and ${ }^{15} \mathrm{O}$, this is not essential for ${ }^{18} \mathrm{~F}$ radiochemistry. Additionally, a substantial number of new drugs contain a $\mathrm{F}$ atom in their structure, increasing the interest of drug companies to use ${ }^{18} \mathrm{~F}$-PET to study their in vivo properties. ${ }^{61}$ Furthermore, the half-life of ${ }^{18} \mathrm{~F}$ matches well with the pharmacokinetics of many small biomolecules. ${ }^{62}$ NPs, however, tend to have longer biological half-lives that are better matched by long-lived PET radionuclides.

Metallic radionuclides elements are attractive candidates for PET applications, particularly for imaging NPs. ${ }^{89} \mathrm{Zr}$, with a long half-life of 3.3 days, has been attached to biomolecules with long circulation times, mainly antibodies for immuno-PET applications. ${ }^{63}{ }^{68} \mathrm{Ga}\left(t_{1 / 2}=67.6 \mathrm{~min}\right)$, due to its generatorbased production (Table 3 ), is increasingly being used for the radiolabelling of peptides and small molecules, making ${ }^{68} \mathrm{Ga}$ the "PET version of ${ }^{99 \mathrm{~m}} \mathrm{Tc}$ ". ${ }^{64}$ However, it has limited applications for in vivo NP imaging studies due to its short halflife. Several other radionuclides with different nuclear and chemistry properties have been also investigated for a variety of PET applications (Table 3).

\subsection{Radionuclides for therapy}

As discussed in the previous section, radionuclides with $\alpha, \beta^{-}$and Auger $\mathrm{e}^{-}$emissions have therapeutic applications (Table 4). The use of radionuclides for therapy is not a novel concept. The treatment of thyroid cancer and hyperthyroidism with thyroidavid ${ }^{131}$ I-iodide was implemented more than 70 years ago. ${ }^{65}$ Other important therapeutic radionuclides used clinically is the bone-tropic ${ }^{223} \mathrm{Ra}$; with demonstrated effectiveness in bone related solid tumours and bone metastases in prostate cancer. ${ }^{66}$ Other emerging radionuclides for therapy are ${ }^{177} \mathrm{Lu}$ and ${ }^{225} \mathrm{Ac}$, being investigated in different clinical trials for theranostics applications in neuroendocrine tumours and prostate cancer. ${ }^{67-69}$

For therapeutic applications with antibodies (radioimmunotherapy), several formulations are also under evaluation using ${ }^{90} \mathrm{Y}$ as a therapeutic radionuclide, with some of them already approved - such as ${ }^{90}$ Y-Ibritumomab tiuxetan (Zevalin ${ }^{\circledR}$ ) used as treatment for non-Hodgkin's lymphoma. ${ }^{70}$ The integration of therapeutic radionuclides into nanomaterials has the potential of not only improving their therapeutic efficiency but also their theranostic capabilities with a broad variety of applications. However, the usual slow excretion of nanomaterials poses a significant barrier for this approach.

\subsection{Theranostic pairs of radionuclides}

Besides the use of individual radionuclides for imaging and/or therapy, certain combinations of radioisotopes can be used as theranostic pairs for both imaging and therapy. These combinations are formed by two radioisotopes of the same chemical element, one with the appropriate radio-physical properties to generate a signal for PET or SPECT detection, and the other isotope with suitable therapeutic properties. This is an interesting approach since both isotopes are radioisotopes of the same element and hence, only one chemical element is ultimately applied allowing both diagnosis and therapy.

The first example of a theranostic pair application was described in 1993 by Herzog et al. where the pair ${ }^{86} \mathrm{Y} /{ }^{90} \mathrm{Y}$ was studied to evaluate, in a patient with bone metastases, the pharmacokinetics of the radiotracer ${ }^{86} \mathrm{Y}$-citrate as an analogue of the radiotherapeutic ${ }^{90} \mathrm{Y}$-citrate. ${ }^{71}$ Since then, different pairs have been proposed increasing the opportunities in personalised medicine. Theses pairs are formed by $\beta^{+}$or $\gamma$-emitters for PET or SPECT respectively, in combination with radionuclides with $\alpha, \beta^{-}$and Auger $\mathrm{e}^{-}$emissions for the therapeutic response. Some of the most important proposed pairs are: ${ }^{72} \mathrm{As} /{ }^{77} \mathrm{As}$, 
${ }^{64} \mathrm{Cu} /{ }^{67} \mathrm{Cu},{ }^{68} \mathrm{Ga} /{ }^{67} \mathrm{Ga},{ }^{124} \mathrm{I} /{ }^{131} \mathrm{I},{ }^{110 \mathrm{~g}} \mathrm{In} /{ }^{111} \mathrm{In},{ }^{44 \mathrm{~g}} \mathrm{Sc} /{ }^{47} \mathrm{Sc},{ }^{83} \mathrm{Sr} /{ }^{89} \mathrm{Sr}$, ${ }^{152} \mathrm{~Tb} /{ }^{161} \mathrm{~Tb},{ }^{152} \mathrm{~Tb} /{ }^{149} \mathrm{~Tb}$ and ${ }^{86} \mathrm{Y} /{ }^{90} \mathrm{Y} .{ }^{72}$

The nature of NPs offers unique possibilities in combination with theranostic radionuclide pairs, such as the ability of co-loading radionuclides and drugs with synergistic therapeutic properties. However, as mentioned in the previous section, the slow biological excretion profile of most nanomaterials represents a significant barrier towards the clinical translation of radionuclide-based therapeutic nanomaterials.

\subsection{Biodistribution of free radionuclides}

A key factor when in vivo studies are conducted with radiolabelled nanomaterials is the biodistribution of the "free" or unchelated radionuclide. Although this is often underestimated, the lack of consideration of this aspect can easily lead to misinterpreting imaging signal: wherein the biodistribution of the free radionuclide is wrongly attributed to the nanomaterial signal. On the contrary, knowledge of the radionuclide biodistribution can also aid the selection of the most appropriate radionuclide depending on the final application; to avoid, as far as possible, the overlapping between the signals of the free radionuclide and the radiolabelled nanomaterial. It is worth noting that this is mostly applicable when the radiolabeled NP releases its radionuclide in its 'free' form. When radionuclides are chelated to a well-suited small molecule-based ligand/chelator it is expected that release of this component from the NP structure will result in fast excretion via the renal excretion pathway, unless any biological process that may be involved in NP degradation affects the expected radiometal-chelator stability.

Table 5 shows the biodistribution of the most important radionuclides used for the radiolabelling of nanomaterials. It is important to note that this table highlights the organs where an unchelated radionuclide can be found in a qualitative manner. The degree of uptake will depend on the type of specimen, experimental model and the biodistribution time. In addition, some radionuclides are often produced under different formulations (e.g. ${ }^{89} \mathrm{Zr}$ can be used as $\left[{ }^{89} \mathrm{Zr}\right] \mathrm{ZrCl}_{4}$ or $\left[{ }^{89} \mathrm{Zr}\right] \mathrm{Zr}$-oxalate) with possible effects over the biodistribution, the chemical identity of the free radionuclide is defined in the table.
It is particularly worth highlighting that several radionuclides show high uptake in organs where nanomaterials commonly accumulate (e.g. liver), and this should be taken into account when analysing the images. In summary, there are different factors affecting the radionuclide choice. These involve the type of production, the radio-physicochemical properties and the biodistribution. The selection of the radionuclide usually delimits the type of radiolabelling method, although different methods for the same radionuclide can be applied as further described in the next sections.

\section{Radiolabelling nanomaterials: basic concepts and methods}

\subsection{Basic concepts}

In this section we will introduce and summarise basic radiochemical concepts which are widely applicable to any radiolabelling chemistry. However, we will place a particular emphasis on those aspects that are relevant to the radiochemistry of nanoparticles.

4.1.1 Radiotracer. A radioactive tracer, or radiotracer, is a chemical compound where at least one element is radioactive, making it traceable by the detection of radionuclide decay. This term is usually applied to small radiopharmaceuticals and often related with a very low concentration of a radiolabelled substance.

4.1.2 Radiolabelled nanoparticle. Although a radiotracer by definition, a radiolabelled NP can be defined as a nanomaterial that stably carries a radionuclide as part of its structure. Unlike with most small-molecule radiotracers, the presence of the radionuclide in NPs most often represents a negligible modification to their original structure. This is due to the large size of NPs and the small amounts of radionuclides per NP required for efficient SPECT/PET imaging (low specific activity; vide infra). It is still an important factor to take into account, as some radiolabelling modifications have been shown to affect the physicochemical properties of NPs (vide infra). Hence, radiolabelling strategies must preserve the integrity of the nanomaterial without

Table 5 Biodistribution of free/unchelated radionuclides. Adapted with permission from ref. 73

\begin{tabular}{|c|c|c|c|c|c|c|c|c|c|c|c|c|}
\hline \multirow[b]{2}{*}{ Radionuclide } & \multicolumn{11}{|c|}{ Qualitative biodistribution of "free" radionuclides } & \multirow[b]{2}{*}{ Ref. } \\
\hline & Blood & Liver & Kidneys & Heart & Spleen & Bone & Pancreas & Salivary glands & Thyroid & Stomach & Tumour & \\
\hline${ }^{111} \operatorname{In}\left({ }^{111} \mathrm{InCl}_{3}\right)$ & & 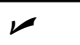 & レ & & & レ & & & & & 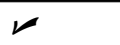 & 74 \\
\hline 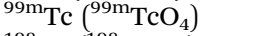 & & & & & & & & & 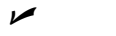 & $\nu$ & & 75 \\
\hline${ }^{198} \mathrm{Au}\left({ }^{198} \mathrm{AuCl}_{4}\right)$ & 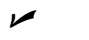 & $\nu$ & レ & & & & & & & & & 76 \\
\hline${ }^{18} \mathrm{~F}\left(\mathrm{Na}^{18} \mathrm{~F}\right)$ & & & & & & $\mathscr{\nu}$ & & & & & & 77 \\
\hline${ }^{67 / 68} \mathrm{Ga}\left({ }^{67} \mathrm{Ga}\right.$-citrate $)$ & レ & $\nu$ & レ & & & $\mathscr{L}$ & & & & & $\nu$ & 78 and 79 \\
\hline${ }^{\text {radio }} \mathrm{I}\left(\mathrm{Na}^{\text {radio }} \mathrm{I}\right)$ & & & & & & & & 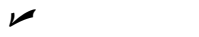 & 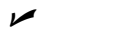 & $\nu$ & & 80 \\
\hline${ }^{64} \mathrm{Cu}\left({ }^{64} \mathrm{CuCl}_{2}\right)$ & & $\nu$ & & & & & & & & & 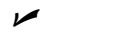 & 81 and 82 \\
\hline${ }^{89} \mathrm{Zr}\left({ }^{89} \mathrm{ZrCl}_{4}\right)$ & & & & & & $\nu$ & & & & & & 83 \\
\hline${ }^{52} \mathrm{Mn}\left({ }^{52} \mathrm{MnCl}_{2}\right)$ & & $\nu$ & $\nu$ & $\nu$ & & & $\nu$ & $\nu$ & & & 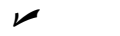 & 84 \\
\hline${ }^{90} \mathrm{Y}\left({ }^{90} \mathrm{YCl}_{3}\right)$ & & $\nu$ & $\nu$ & & & $\nu$ & & & & & & 85 \\
\hline${ }^{177} \mathrm{Lu}\left({ }^{177} \mathrm{LuCl}_{3}\right)$ & & & & & & 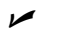 & & & & & & 86 \\
\hline${ }^{188} \mathrm{Re}\left({ }^{188} \mathrm{ReO}_{4}\right)$ & $\nu$ & & & & & & & レ & レ & 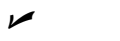 & レ & 87 \\
\hline${ }^{223} \mathrm{Ra}\left({ }^{223} \mathrm{RaCl}_{2}\right)$ & & & 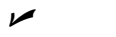 & & 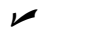 & $\nu$ & & & & & & 88 \\
\hline${ }^{225} \mathrm{Ac}\left({ }^{225} \mathrm{AcCl}_{3}\right)$ & & レ & & & & $\nu$ & & & & & & 89 \\
\hline
\end{tabular}


altering the original physicochemical properties, biodistribution or pharmacokinetics (see Sections 4.2-4.4)

4.1.3 Specific activity and molar activity. The specific activity of a radiotracer is the measured activity per gram of compound, whilst the molar activity is defined as the measured activity per mole of compound. ${ }^{90}$ Inside both definitions, it is important to specify the time of the measurement in order to correct the radionuclide decay. Thus, these terms provide a measure of the radioactivity in a certain amount of substance and very importantly, relate the amount of a radiolabelled material with the dose to dispense. The higher specific or molar activity the lower the dose required to reach the same activity. This is not only important for imaging studies but also for therapeutic applications where the amount of the injected activity is related with the therapeutic efficiency. Therefore, a high specific/molar activity ensures enough levels of activity with low radiotracer amounts, allowing microdosing clinical studies, highly recommended by the FDA for the pre-evaluation of new drugs, due to the low risk profile. ${ }^{91}$

4.1.4 Carrier-added (c.a) and non-carrier added (n.c.a) radionuclides. These terms, comprehensively discussed by Goeij et al., are related to the specific activity of a radionuclide. ${ }^{92}$ Thus, the term carrier-added refers to radionuclides where not only the radionuclide but also the stable element or other inactive material are present, hence decreasing the specific activity. The term non-carrier added is used when the radionuclide is carefully produced to avoid the presence of the stable element and other substances are not required. A third term, named carrier-free, is often use when the radionuclide reaches the theoretical specific activity (i.e. $100 \%$ of isotopic abundance). However, it is recommended to avoid this term since conventional radionuclides always present side contaminations with other elements and thus, are never carrier-free.$^{93}$ It is clear that non-carrier added radionuclides have higher specific activities and purity than carrier-added radionuclides. Therefore, noncarrier added radionuclides are preferred for a radiolabelling reaction.

4.1.5 Radiochemical yield (RCY), radiochemical purity (RCP) and radiochemical stability (RCS). These terms will be frequently used over the next sections. The radiochemical yield (RCY) is defined as "the amount of activity in the product expressed as the percentage (\%) of starting activity used in the considered process (e.g. synthesis, separation, etc.)". ${ }^{90}$ This is essentially the same concept as chemical yield in any "cold" or non-radiochemical reaction. Here, the efficiency of the reaction is measured by the level of activity, assigned to a single radionuclide, present in the material with respect to the starting activity used for the radiolabelling. This definition logically assumes that the activity is decay corrected to the start of the reaction, and the measured activity is referring to the same radionuclide. The radiochemical purity (RCP) measures the presence of other radiochemical species within a sample. In this regard, a high RCP means the absence of other radioactive sources and hence, a high radio-pure substance. Noteworthy, this parameter is a measurement of the radioactive purity with no significance over the presence of other non-radioactive species. High RCP in nanomaterials is often reached due to the simplicity of the purification protocols, that are mainly based on the size difference between the nanomaterial and the radionuclide (size-exclusion or ultrafiltration purification protocols) or based on the NP physicochemical properties (e.g. magnetic separation protocols). Another important parameter is radiochemical stability (RCS), that provides a measurement of the strength of the nanoparticle-radionuclide bond after the radiolabelling reaction. For applications in imaging and therapy, the RCS is usually the ex vivo measurement of the stability under simulated in vivo conditions (i.e. human serum or PBS at $37^{\circ} \mathrm{C}$ ). This is of a paramount importance to analyse whether a radionuclide leaks from the NP in a scenario which may lead to the misinterpretation of the results. As discussed in the next sections, an appropriate radiolabelling strategy must render radiolabelled nanomaterials with high RCP and RCS. Moreover, methods providing high RCYs are always desirable in order to obtain high specific or molar activities of highlighted importance in theranostic applications.

\subsection{Chelator-based radiolabelling}

The labelling of compounds with non-metallic radionuclides (e.g. fluorine-18, carbon-11 and iodine-131, etc.) is achieved by direct covalent bond formation (see Section 4.4.3 for further details). However, radionuclides with metallic character (radiometals; e.g. copper-64, technetium-99m, zirconium-89) often require the use of chelators and hence coordination chemistry approaches to efficiently attach them to the NP of interest. The purpose of a chelator is to bind the radiometal ion through two or more bonds creating highly stable metal complexes and hence RCS. Due to the 'always on' nature of imaging contrast using nuclear imaging, any radiometals which are not stably bound may distribute differently in vivo causing misleading signal within the images. For this reason, the choice of chelator used with any particular radiometal is of paramount importance.

Understanding the coordination chemistry of the chosen radiometal is essential to avoid the incorrect selection of a chelator. Firstly, the geometric preferences and coordination number will be affected by the atomic number, radii and charge. Additionally, the 'hardness' of the metal ion in terms of Pearson's acid-base concept must be assessed, with the chosen ligand having the appropriate hard/soft donor atoms and with the right electronic properties to improve the kinetic inertness of the complex. In terms of thermodynamic stabilities, polydentate ligands form stable complexes over their monodentate counterparts due to the "chelate effect". This is, in a simplified way, due to the increase in entropy resulting from the complexation of a polydentate ligand and metal ion, as compared with multiple monodentate ligands. Polydentate ligands are usually split into two categories: acyclic/linear chelators and macrocyclic chelators. Acyclic or linear chelators often benefit from rapid radiometal complexation due to their lack of rigidity. This is in contrast to macrocyclic chelators, which have a relatively rigid and pre-organised structure resulting in higher complex stability (i.e. macrocyclic effect) but suffer from slow complexation kinetics, resulting in the need for high 


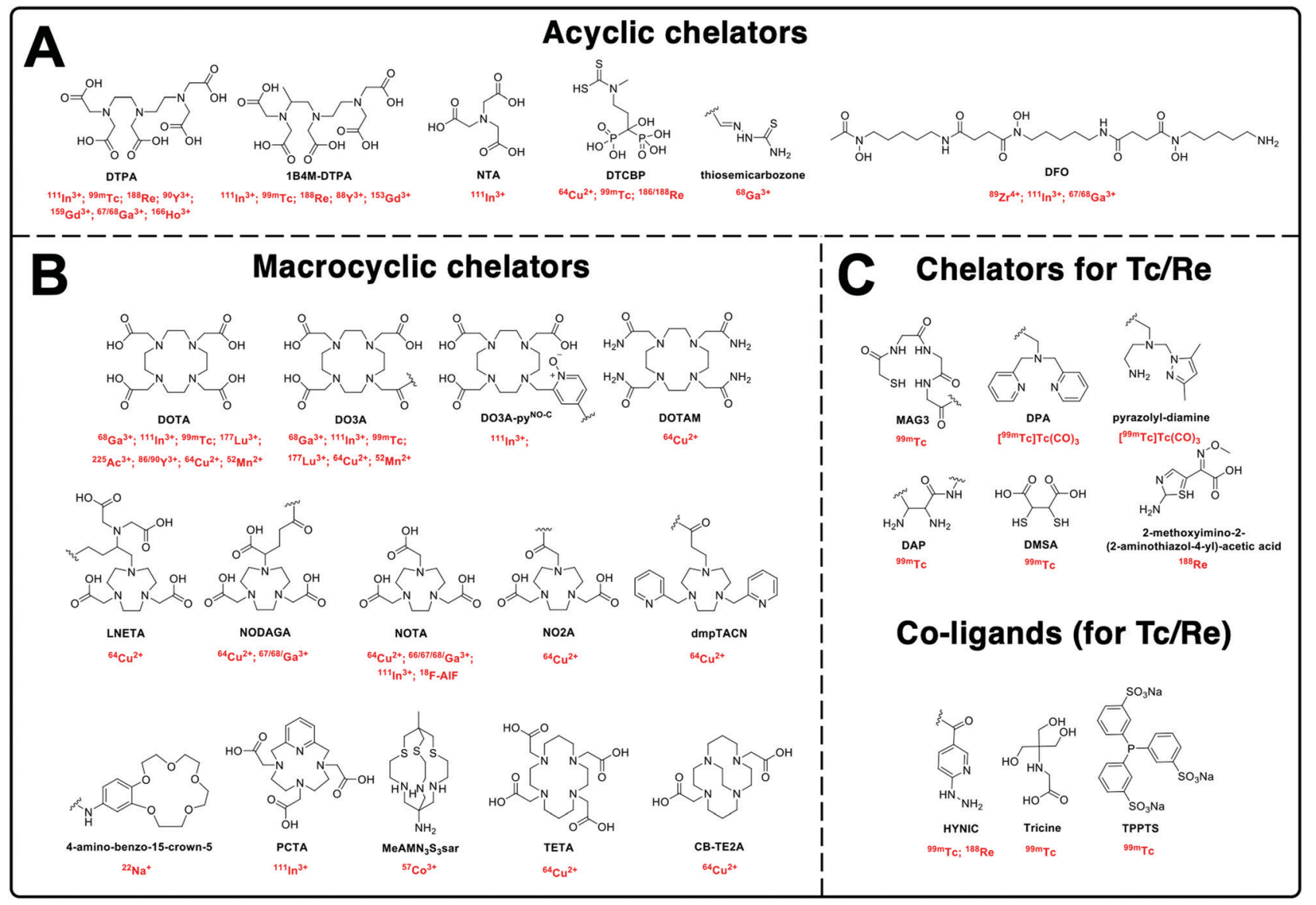

Fig. 6 Chemical structures of the chelators used for radiolabelling nanomaterials described in this review with their corresponding radionuclide(s).

temperatures and long reaction times. This last requirement may be damaging for some heat-sensitive NP types (e.g. protein-based, exosomes). For this reason, the radiolabelling of heat-sensitive NPs with macrocyclic chelators is often done post-complexation via the use of bifunctional chelators (vide infra, Section 4.2.1).

Based on the above principles, an ideal chelator should allow rapid, quantitative complexation under mild conditions (aqueous solvent, room temperature and neutral $\mathrm{pH}$ ), whilst demonstrating high kinetic inertness and thermodynamic stability in biologically relevant medium (i.e. serum). This stability should be for an appropriate amount of time to allow imaging and is usually based on the half-life of the radiometal and pharmacokinetics of the NP of interest. Several reviews have discussed optimised chelators for each radiometal in great detail, and are highly recommended for further reading. ${ }^{94-96}$ Fig. 6 shows the chemical structures of all chelators used for the radiolabelling of NPs discussed in this review, with their corresponding radionuclide(s).

4.2.1 Use of bifunctional chelators. The use of bifunctional chelators is a ubiquitous part of metal-based radiochemistry, and widely exploited for the radiolabelling of NPs. A bifunctional chelator is a compound containing a chelating ligand with a reactive functional group (Fig. 7A) that allows it to be covalently attached (conjugated) to a biologically relevant vector (e.g. protein, peptide). ${ }^{94}$ In the context of the radiolabelling of NPs, an ideal bifunctional chelator allows the stable chelation of

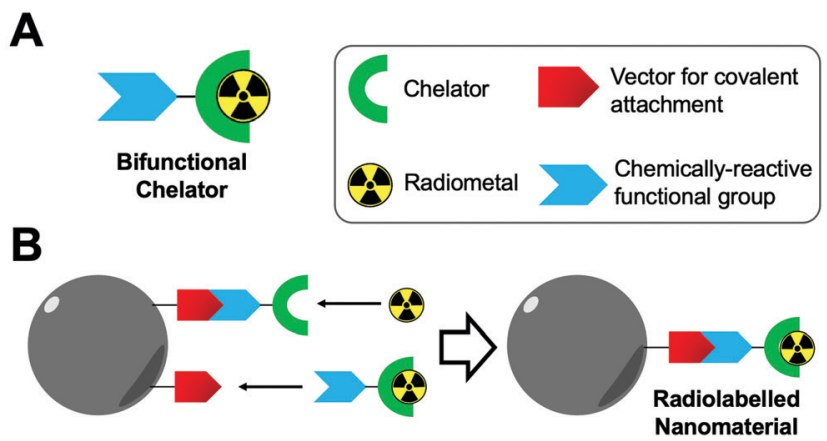

Fig. 7 (A) Schematic representation of a bifunctional chelator. (B) Schematic representation of the radiolabelling of nanoparticles using bifunctional chelators.

the chosen radiometal and can easily be covalently linked to one of the components of the NP (Fig. 7B), often on the surface, via appropriate bioconjugation reactions. There are several standard bioconjugation reactions used commonly with bifunctional chelators (Fig. 8), comprehensively reviewed in the excellent book by G. Hermanson. ${ }^{97}$ These reactions allow selective conjugation, forming covalent links that are stable in physiological medium.

Common chemical functional groups present on the surface of nanomaterials can be radiolabelled using bifunctional chelators. For example, amines can be reacted with chelators containing NHS 


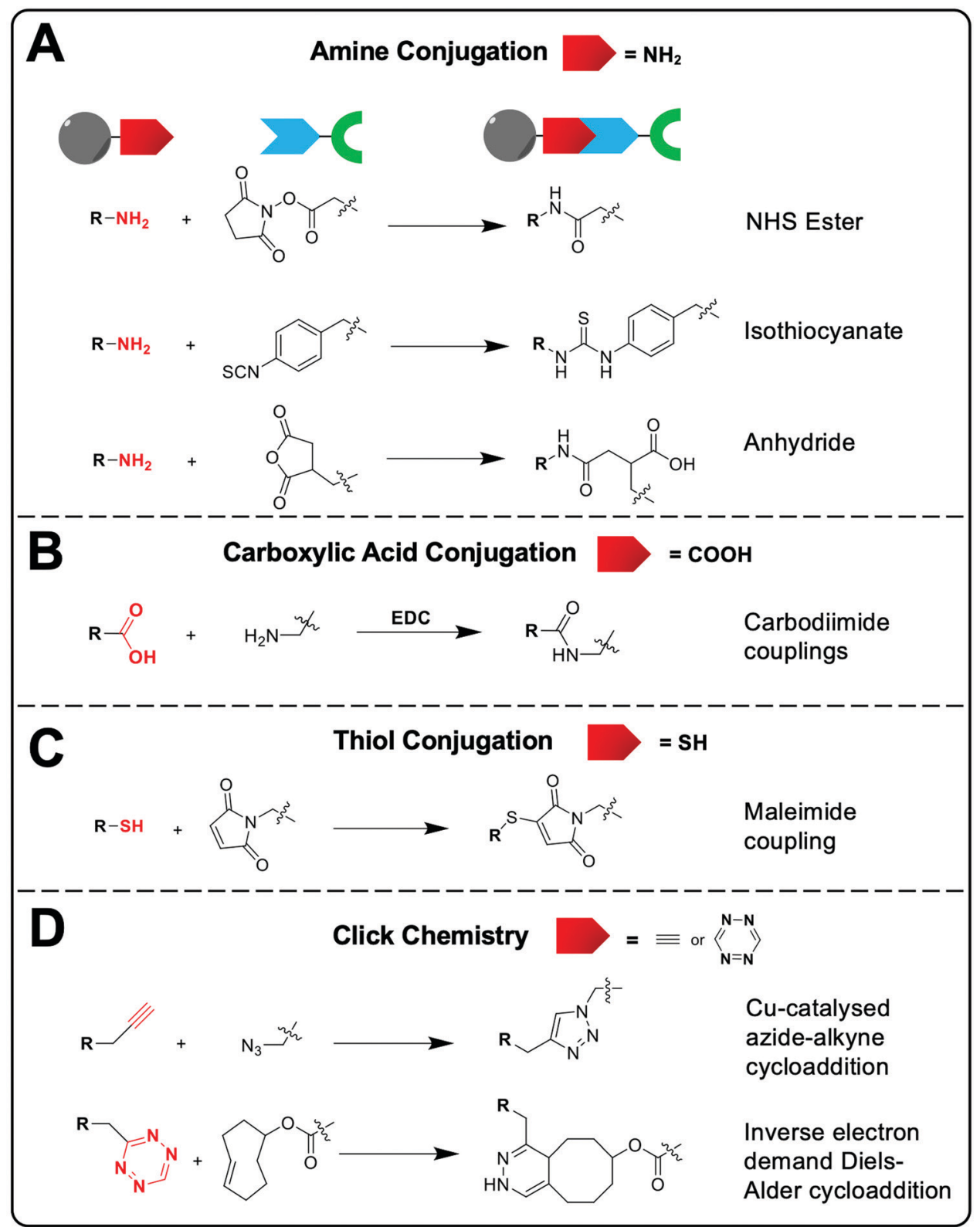

Fig. 8 Common bioconjugation reactions that allow the attachment of bifunctional chelators to the nanomaterial surface. (A) Amine-based conjugation, (B) carboxylic acid-based conjugation, (C) thiol-based conjugation and (D) click chemistry conjugation.

ester groups or cyclic anhydrides to form amide bonds, or with aryl isothiocyanate groups to form isothioureas (Fig. 8A). Carboxylate functionalised NPs can be reacted with amines via the use of carbodiimide coupling reagents, such as EDC, and free thiols can be conjugated using maleimides (Fig. $8 \mathrm{~B}$ and C). ${ }^{98}$ Finally, click chemistry is often used due to its rapid, high yielding reactions. Two commonly used reactions are the copper-catalysed azidealkyne (CuAAC) and inverse electron demand Diels-Alder cycloaddition between a tetrazine and trans-cyclooctene (Fig. 8D). ${ }^{99}$ These reactions have previously been discussed in the context of bifunctional chelators for radionuclide imaging in reviews that are highly recommended for further reading. ${ }^{99-101}$

The selection of the appropriate bioconjugation reaction may be often dictated by the nanomaterial of interest. For example, poly(amidoamine) (PAMAM) dendrimers or lipids used to formulate vesicles will often contain free amine groups capable of easily being reacted with appropriate functional groups. Additionally, polymer-based or polymer-coated and protein-based NPs will often intrinsically contain functional groups for bioconjugation (e.g. carboxylate groups on dextran or aspartic/glutamic amino acids). However, whilst the target vector for conjugation is often intrinsic to the NP formulation, the NP can also be modified to facilitate conjugation of the bifunctional chelator if need be.

4.2.2 Radiometal complex trapping during nanoparticle formation. Another chelator-based method for the radiolabelling of NPs involves the trapping of radiometal complexes during the synthesis of NPs. The complexation of the chosen radiometal with a suitable chelator is first performed, which is then added to the 
Radiolabel complex trapping during formulation

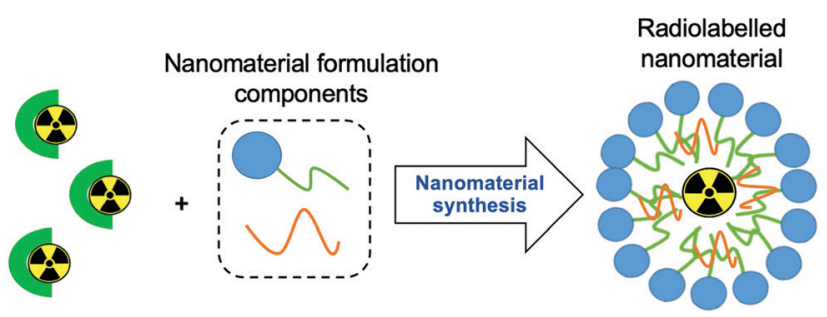

Fig. 9 Schematic representation of the radiometal complex-trapping radiolabelling strategy. Radiometal complexes are added to the mixture during the formation of the nanomaterial and are then subsequently incorporated into the nanoparticle and become trapped.

reagents used for the NP formation. During the synthesis of the NP the radiometal complex will then become trapped, generating the radiolabelled NP (Fig. 9). Whilst this radiolabelling procedure is arguably simple, a major drawback is that the synthesis, and subsequent purification, of the NP must be compatible for radiolabelling. For example, a lengthy process of NP formation will limit the use of short-lived radionuclides, even if they are more appropriate for the imaging application. Additionally, the choice of radiometal complex may depend on the NP being used. For example, a lipophilic radiometal complex may be more favourable for trapping within NPs containing lipophilic pockets (e.g. polymeric micelles). Furthermore, sufficient stability of the radiometal complex during the NP formulation process - and subsequent purification - is necessary to allow incorporation of the radionuclide into the NP.

\subsection{Ionophore-based radiolabelling}

Whilst technically involving chelators, ionophore-based methods are distinct enough from the classic chelator-based methodologies described previously (Section 4.2). Although the following radiolabelling methods are only relevant for vesicle-based NPs (e.g. liposomes, exosomes) containing lipid membranes, they represent a significant portion of the NP literature. Hence, for the sake of clarity, we have separated these methods from the chelator-based methods described above. Fig. 10 summarises the strategies used for ionophore-based NP radiolabelling, that are discussed below.

4.3.1 Ionophore-chelate binding. The term 'ionophore' refers to a ligand which can reversibly bind to a metal ion forming a lipophilic complex which is capable of crossing lipid membranes (Fig. 11A). ${ }^{102}$ This metal-ionophore complex will then release the metal inside the vesicle where it can be transchelated. In the context of radiolabelling NPs, ionophores can be used to radiolabel vesicle-based NPs (e.g. liposomes, exosomes/extracellular vesicles; Sections 4.5.1 and 4.5.2) containing a lipid bilayer membrane. The ionophore ligand will form a complex with the radiometal (referred here throughout as a radio-ionophore) and transport the radionuclide across the lipid bilayer. Importantly, once inside the vesicle, the radio-ionophore complex releases the radiometal where it binds to stably-chelating molecules present in the vesicle core becoming 'trapped' (Fig. 10A). These metal-binding molecules may take the form of chelators (as in Section 4.2), added during formulation of the NP, or may be intrinsic to the NP; such as proteins/nucleic acids present in exosomes (see Section 4.5.2) or drugs present in liposomal nanomedicines (Section 4.5.1). A key benefit of using this method, is that radiolabelling occurs within the NP core - which can result in higher RCS as compared with NPs labelled on their surface. Due to this twostep loading, followed by chelation mechanism, there are three key considerations for radiolabelling vesicles in this way. Firstly, the loading efficiency of the ionophore ligand with the chosen radiometal must be considered - that is, how much of radio-ionophore is loaded into the vesicle. Secondly, the

\section{A lonophore-chelator binding}
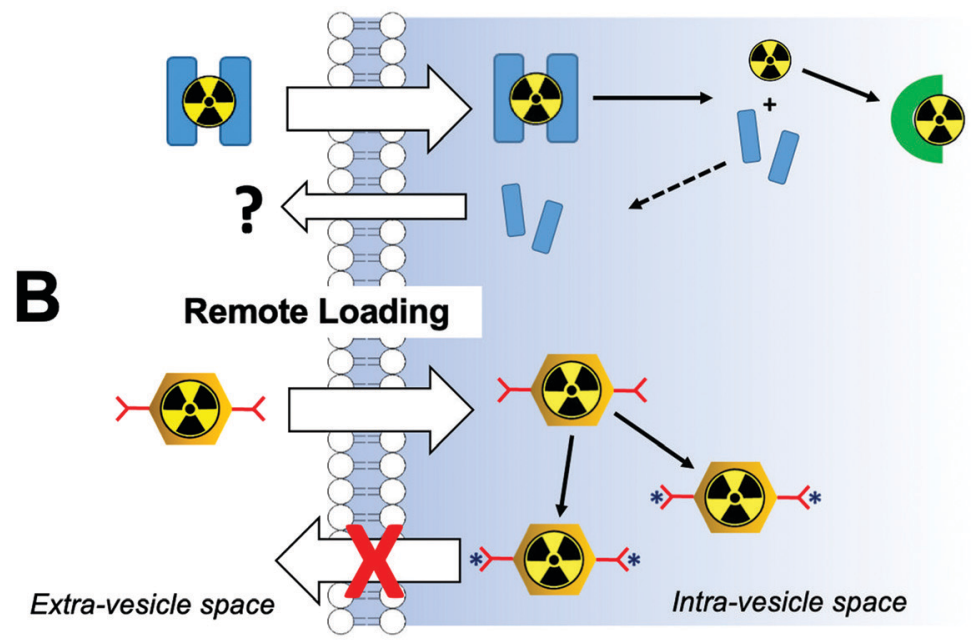

Lipid Membrane

lonophore ligand

Radiometal

Chelator/drug/protein

Radio-ionophore

Radiotracer

Charged radiotracer

Fig. 10 Schematic representation of $(A)$ ionophore-based radiolabelling strategies and (B) remote loading radiolabelling 
A Ionophores
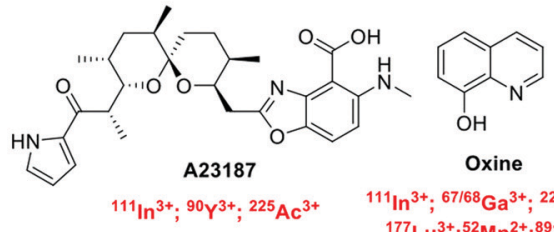

${ }^{11} / \mathrm{In}^{3+} ;{ }^{67 / 68} \mathrm{Ga}^{3+} ;{ }^{225} \mathrm{Ac}^{3+}$ ${ }^{177} \mathrm{Lu}^{3+} ; 52 \mathrm{Mn}^{2+} ; 89 \mathrm{Zr}^{4+}$;
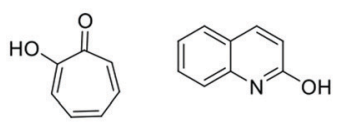

Tropolone

${ }^{111} \mathrm{In}^{3+} ;{ }^{67} \mathrm{Ga}^{3+} \quad{ }^{64} \mathrm{Cu}^{2+} ;{ }^{89} \mathrm{Zr}^{4+}$
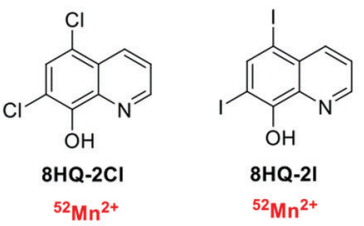

B
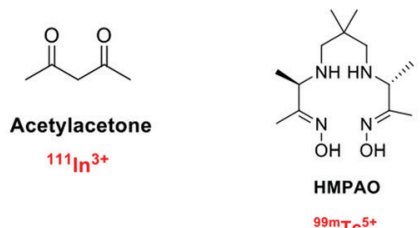

Remote loading

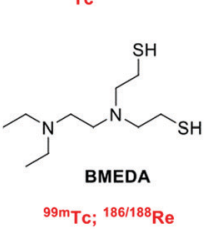

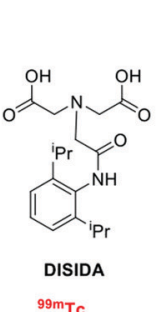
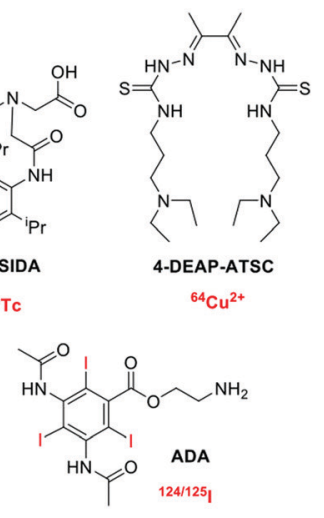

Fig. 11 Chemical structures of (A) common ionophores used in ionophore-chelate vesicle radiolabelling with the corresponding radionuclides; and (B) chemical structures of compounds used for remote loading with their corresponding radionuclides.

radio-ionophore must be sufficiently unstable to release the radiometal within the vesicle. To achieve these two points, the coordination chemistry of the radiometal must be carefully considered. To achieve the formation of the metastable complexes, non-macrocyclic low denticity chelators are often used (Fig. 11A) with a mixture of hard- and softer-donor atoms. Finally, the chelating molecule inside the vesicle must allow the rapid formation of highly stable complexes with the released radiometal in mild, physiological conditions - which are usually often present within vesicles.

4.3.2 Remote loading. Similarly to ionophore-chelate radiolabelling, remote loading involves the use of lipophilic radiotracers capable of passively crossing lipid membranes on vesicle-based NPs (Fig. 10B and 11B). However, this technique differs in two key aspects. Firstly, the radiometal complex/ radiotracer is designed to be sufficiently stable to stay intact inside the vesicle core. Secondly, the complex must contain functional groups capable of becoming charged in the aqueous environment of the vesicle core, causing the trapping of the complex by decreasing its lipophilic solubility (Fig. 10B). This trapping can occur passively or in some specific cases can be increased by the presence of other compounds in the vesicle core (see Section 4.5.1). Based on these mechanisms, an ideal remote loading compound should allow high loading efficiencies, whilst also being sufficiently stable and capable of being trapped within the vesicle core long enough to allow in vivo imaging.

\subsection{Non-chelator radiolabelling}

Non-chelator based strategies involve the direct incorporation of radionuclides into the core and/or surface of nanomaterials, circumventing the need for external chelating agents. Hence, these methods are usually more straightforward and less timeconsuming than chelator-based methods - though this is dependent on the type of nanomaterial and radionuclide being used. Removing the use of chelators will often decrease the number of reaction steps and most importantly, preserve the integrity of the nanomaterial by avoiding the bulky chelator molecule that could affect the in vivo behaviour. ${ }^{103}$ Nonchelator based strategies adapt a variety of common radiolabelling reactions, as well as implementing bespoke radiolabelling methods specifically designed for the integration of radionuclides into nanomaterials (Fig. 12).

Traditional radiochemical reactions such as radiohalogenations, ${ }^{11} \mathrm{C}$-methylations or chemical adsorptions are often used. In addition, reactions such as the use of hot + cold NP precursors or proton beam activation of materials are specific for nanomaterials. Other non-standard radiochemical labelling methods such as those based on radioisotopic exchange or physical interactions take the advantage of the physicochemical properties of certain nanomaterials to facilitate radiolabelling. Each of the non-chelator based NP radiolabelling methods will now be discussed in detail.

4.4.1 Mixing hot + cold precursors. In this NP radiolabelling approach, a mixture of starting reagents containing the radionuclide and the non-radioactive (or 'cold') nanomaterial precursors are reacted to provide the radiolabelled nanomaterial in a single step (Fig. 13).

This strategy, exclusive for inorganic nanomaterials, is often straightforward with fast protocols, making this method the most widely used of the non-chelator NP radiolabelling methods. From a chemical point of view, this method is based on the radiochemical doping of the nanomaterial during synthesis. The radionuclide (hot precursor) is added in trace levels to the nanomaterial precursors (carrier-added) triggering a co-precipitation that leads to the incorporation of the radionuclide into the crystal lattice of the nanostructures. ${ }^{104}$ The 'doping' represents the main advantage of this strategy, as it maintains the nanomaterial's structural integrity, whilst allowing strong radiochemical stabilities. This is particularly the case with homo-radionuclide doping, (i.e. the nanomaterial core contains the same element as the radionuclide dopant) which allows imaging of the in vivo fate of some nanomaterials without modifications to the NP structure. For instance, diverse 


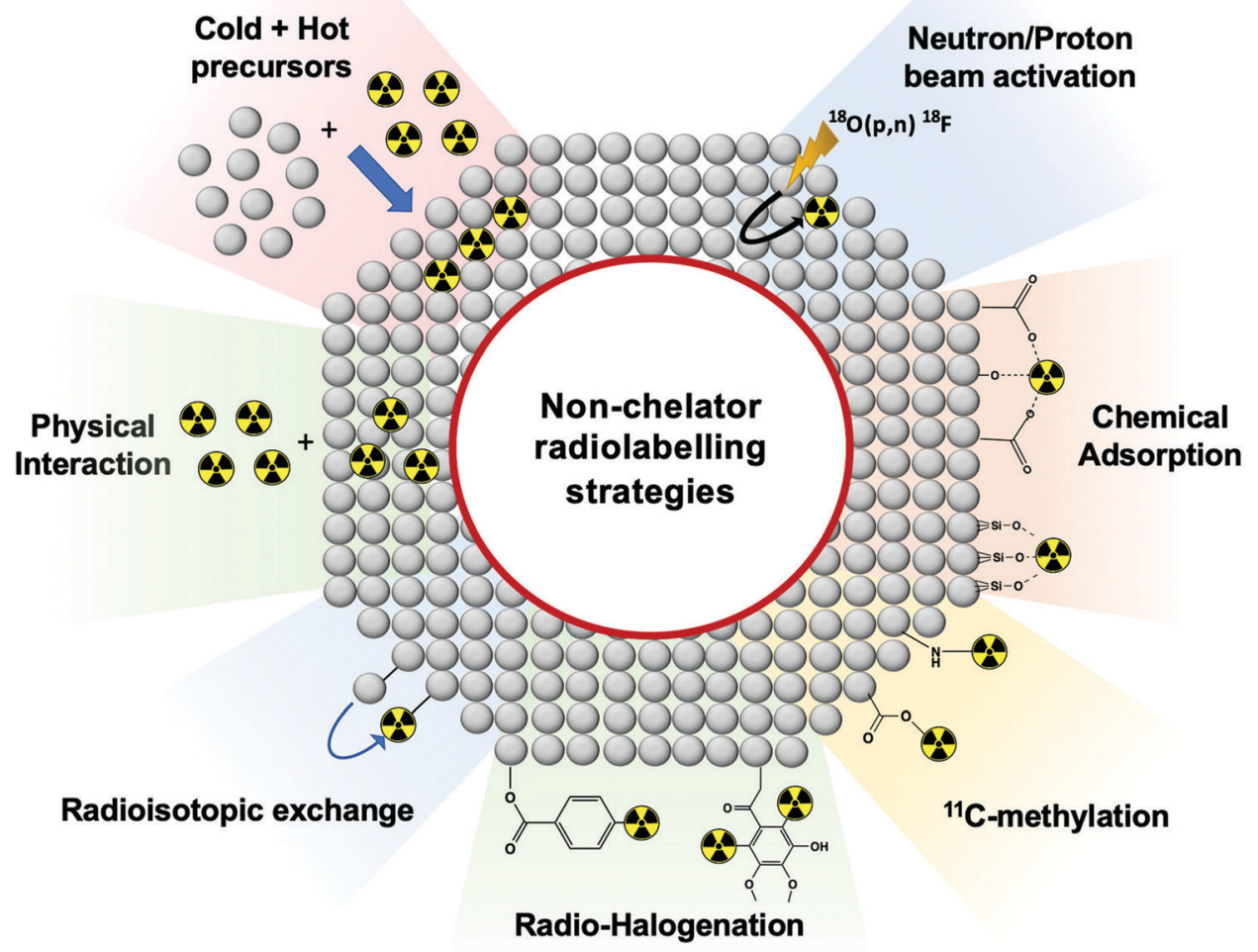

Fig. 12 Schematic representation of non-chelator based radiolabelling methods.

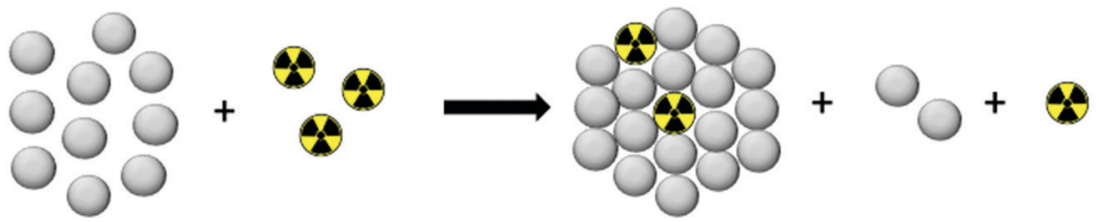

Fig. 13 Representation of hot + cold precursors radiolabelling strategy.

gold NPs have been doped with ${ }^{195} \mathrm{Au},{ }^{198} \mathrm{Au}$ or ${ }^{199} \mathrm{Au}$ or iron oxide NPs with ${ }^{59} \mathrm{Fe}$ for similar purposes. ${ }^{105-111}$ For example, Zhao et al. doped Au NPs with ${ }^{199} \mathrm{Au}$ to study the biodistribution in tumour-bearing mice model after conjugation with D-Ala1peptide T-amide (DAPTA) (Fig. 14A). SPECT/CT imaging experiments revealed the elimination of the $\left[{ }^{199} \mathrm{Au}\right] \mathrm{AuNPs}$ by liver and spleen with specific accumulation in the tumour due to the DAPTA vectorisation (Fig. 14B). ${ }^{106}$

There are some considerations in order to achieve high RCYs with this strategy. A high solubility between both, cold and hot, precursors is required. Considering most of radionuclides are delivered in aqueous solutions, this strategy is then limited to reactions conducted in water. It is also important to control the ionic strength of the reaction media to allow the nucleation and growth of the nanomaterial. The physicochemical properties of the radionuclide also play a key role. The ionic radius of the radionuclide and its corresponding non-radioactive ion should be similar. In addition, the radionuclide should have the same ionic charge, in order to coordinate with the intermediate complex formed by the cold precursors before nucleation. Considering this, mainly metallic cations can be integrated into NPs using this strategy with few suitable radionuclide-NP pair choices (Table 6). For instance, IONPs were doped with ${ }^{68} \mathrm{Ga}$ for tumour imaging driven by the functionalisation with an RGD peptide ( ${ }^{68}$ Ga-C-IONP-RGD, Fig. 14C). PET/CT imaging showed high accumulation in the tumour $1 \mathrm{~h}$ after the injection of the ${ }^{68} \mathrm{Ga}-\mathrm{C}$-IONP-RGD with no signals of free ${ }^{68} \mathrm{Ga}^{3+}$ confirming the high stability of the radiolabelling (Fig. 14D). ${ }^{115}$ Other successful combination, reported by Yang et al., is the use of ${ }^{153} \mathrm{Sm}$ as hot precursor for the formation of $\mathrm{NaLuF}_{4}$ UCNPS (Fig. 14E). The biodistribution of ${ }^{153} \mathrm{Sm}-\mathrm{UCNPs}$ was easily addressed by in vivo SPECT/CT imaging revealing a rapid clearance to the liver and spleen $1 \mathrm{~h}$ after the i.v. injection and main accumulation into the spleen after $24 \mathrm{~h}$ (Fig. 14F). 
A

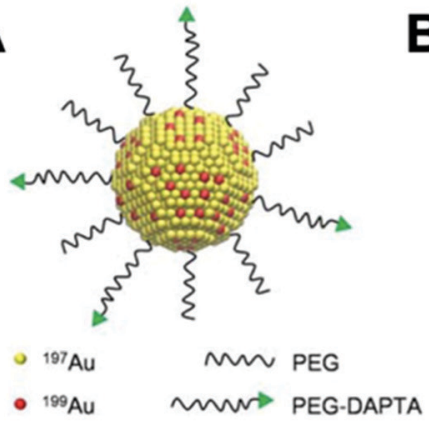

B

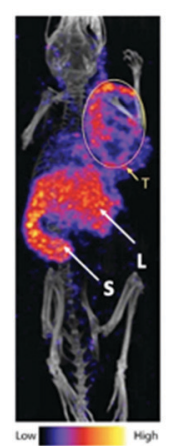

C

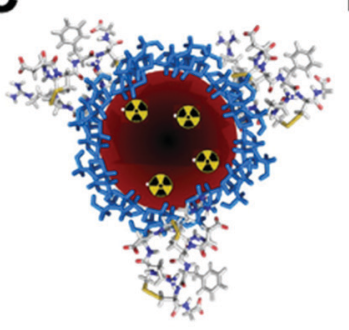

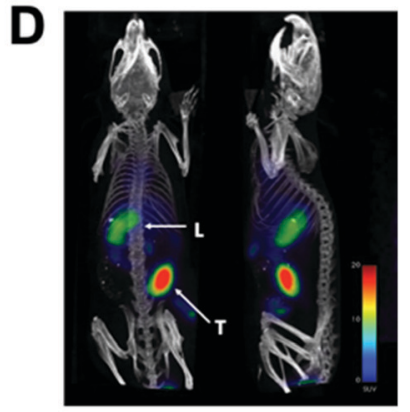

${ }^{68}$ Ga-C-IONP-RGD

$\mathbf{E}$

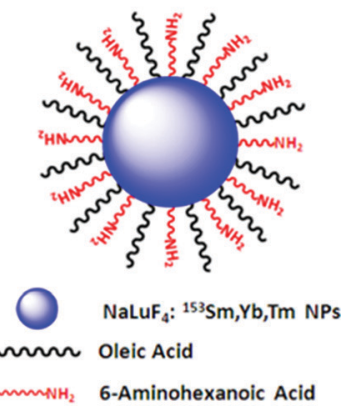

$\mathbf{F}$

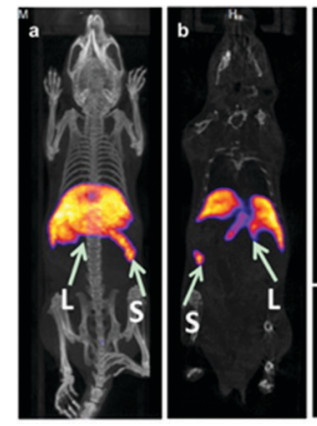

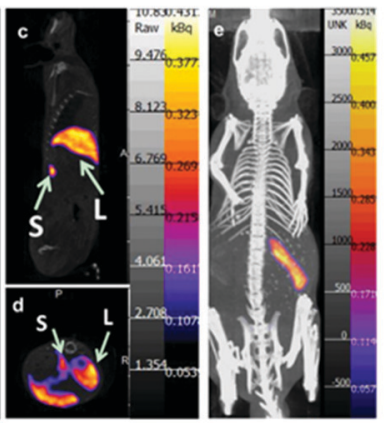

Fig. 14 (A) Radioactive Au nanoparticles doped with ${ }^{199} \mathrm{Au}$ atoms conjugated with D-Ala1-peptide T-amide (DAPTA), (B) NanoSPECT/CT image of a 4T1 tumour-bearing mouse $24 \mathrm{~h}$ post injection of the $5 \mathrm{~nm}{ }^{199} \mathrm{Au}$-AuNP-DAPTA probe (the tumour is labelled by an ellipse in yellow colour. T: tumour, L: liver, S: spleen), (C) ${ }^{68} \mathrm{Ga}$ core-doped iron oxide nanoparticles functionalised with RGD peptide ( ${ }^{68} \mathrm{Ga}-\mathrm{C}-$-IONP-RGD), (D) PET/CT imaging of tumourbearing mice 1 hour after injection of ${ }^{68} \mathrm{Ga}-\mathrm{C}-\mathrm{IONP}-\mathrm{RGD}$, showing strong activity in the tumour (T: tumour, L: liver), (E) the schematic diagram of the NaLuF4: ${ }^{153} \mathrm{Sm}$,Yb,Tm nanoparticles, (F) in vivo SPECT images after intravenous injection of ${ }^{153} \mathrm{Sm}-U \mathrm{CNPs}$. (a) Whole-body three-dimensional projection, (b) coronal, (c) sagittal and (d) transversal images acquired at $1 \mathrm{~h}$ and (e) whole-body three-dimensional projection images acquired at $24 \mathrm{~h}$ are shown respectively. The arrows inset point to the liver $(\mathrm{L})$ and spleen $(\mathrm{S})$. Adapted and reproduced with permission from ref. 112-114.

Table 6 Reported examples of radionuclide-nanoparticle pairs using hot + cold precursors strategy

\begin{tabular}{lll}
\hline Nanoparticle type & Radionuclide & Ref. \\
\hline Iron oxide NPs & ${ }^{225} \mathrm{Ac},{ }^{64} \mathrm{Cu},{ }^{59} \mathrm{Fe},{ }^{68} \mathrm{Ga},{ }^{111} \mathrm{In}$ & $109-111$ and $115-119$ \\
Gold NPs & ${ }^{195} \mathrm{Au},{ }^{198} \mathrm{Au},{ }^{199} \mathrm{Au}$ & $105-108$ \\
Up-converting NPs & ${ }^{153} \mathrm{Sm},{ }^{90} \mathrm{Y}$ & 112 and 120 \\
Quantum dots & ${ }^{109} \mathrm{Cd},{ }^{64} \mathrm{Cu},{ }^{125 m} \mathrm{Te}$ & $121-123$ \\
Cerium oxide NPs & ${ }^{141} \mathrm{Ce},{ }^{65} \mathrm{Zn}$ & 124 \\
Silver NPs & ${ }^{131} \mathrm{I}$ & 125
\end{tabular}

Finally, it is worth highlighting that if the radionuclide and the coating molecule are oppositely charged, the labelling may be conducted by chemical adsorption (see Section 4.4.5) rather than by the radioactive co-precipitation - with possible implications for the radiochemical stability. Being a convenient strategy for pre-clinical purposes, it presents a main limitation for clinical applications since the radionuclide is integrated from the beginning, demanding fast and effective purification protocols to reduce the radioactive exposition to the operator. The potential lack of reproducibility between independent batches could also be a limitation of this strategy which requires extremely reproducible synthetic protocols.

4.4.2 Neutron/proton beam activation. This strategy relies on the bombardment of the nanomaterial with a neutron or a proton. Then, one atom of the nanomaterial undergoes a

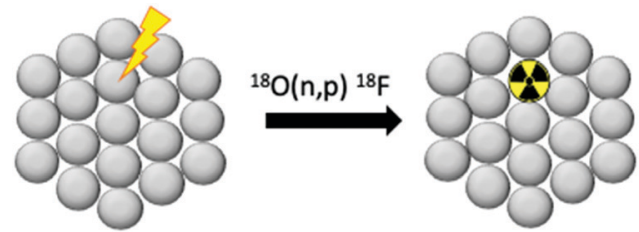

Fig. 15 Schematic representation of neutron/proton beam activation radiolabelling strategy.

nuclear reaction (vide supra) providing the radionuclide in situ (Fig. 15). ${ }^{126}$ This method has been applied for the radiolabelling of ${ }^{18} \mathrm{O}$-enriched $\mathrm{Al}_{2} \mathrm{O}_{3}$ (alumina) NPs by a bombardment with $16 \mathrm{MeV}$ protons transmuting ${ }^{18} \mathrm{O}$ to ${ }^{18} \mathrm{~F}$ via the ${ }^{18} \mathrm{O}(\mathrm{p}, \mathrm{n}){ }^{18} \mathrm{~F}$ nuclear reaction. ${ }^{127}$ Following a similar approach, $\mathrm{Al}_{2} \mathrm{O}_{3}$ NPs were also successfully radiolabelled with ${ }^{13} \mathrm{~N}$ through a ${ }^{16} \mathrm{O}(\mathrm{p}, \alpha){ }^{13} \mathrm{~N}$ proton activation reaction. ${ }^{128}$ In addition to proton activation radiolabelling reactions, neutron activation has been also carried out for the radiolabelling of holmiumbased garnet magnetic NPs (HoIG) via the ${ }^{165} \mathrm{Ho}(\mathrm{n}, \gamma){ }^{166} \mathrm{Ho}$ nuclear reaction and more recently, boron nitride nanotubes (BNNTs) with ${ }^{153} \mathrm{Sm}$ and ${ }^{159} \mathrm{Gd}$ through ${ }^{152} \mathrm{Sm}(\mathrm{n}, \gamma){ }^{153} \mathrm{Sm}$ and ${ }^{158} \mathrm{Gd}(\mathrm{n}, \gamma){ }^{159} \mathrm{Gd}$ nuclear reactions respectively. ${ }^{129,130}$

A high control over the radiolabelling location represents the main advantage of this method, as only specific atoms can 
undergo the nuclear reaction, with a consequently high RCS. However, this method has a key drawback in the requirement of a proton/neutron beam source, which involves the use of complex instruments that are not widely available. Additionally, the high energies used in these nuclear reactions may affect the integrity of sensitive biological species that may be attached to the nanomaterial, limiting the applications to purely inorganic nanomaterials.

4.4.3 Radio-halogenation and ${ }^{11} \mathrm{C}$-methylation. Strategies to incorporate radio-halogen nuclides and ${ }^{11} \mathrm{C}$ into nanomaterials are based on the application of common small-molecule radiochemistry reactions. However, whilst there are few examples of ${ }^{11} \mathrm{C}$ radiolabelled nanomaterials (vide infra), ${ }^{131,132}$ radiohalogenation with long-lived iodine nuclides has been extensively used. For this purpose, different iodination mediators such as iodobeads, iodogen, chloramine-T or the Bolton-Hunter reagent are usually applied (Fig. 16). The first three mediators have been widely used for the radioiodination of tyrosine residues and some derivatives in a vast number of biomolecules. ${ }^{133}$ These are oxidising agents that react with iodine anions yielding electrophilic synthons to further conduct electrophilic substitution in the orthopositions to the phenolic groups on tyrosine residues (Fig. 16A).

These methods are quick, with the radioiodination occurring in seconds to a few minutes and usually in high radiolabelling yields. Chloramine-T is used in solution generating a strong oxidising environment that triggers the radioiodination in just $30 \mathrm{~s}$. Then, subsequent quenching with a reducing agent (usually sodium metabisulfite) is required. Although the chloramine-T method is fast, cheap and reproducible, active biomolecules can be affected by the oxidant and/or the reducing agent. To overcome this limitation, the chloramine-T is immobilised in a polystyrene bead (Iodobead) where the reactivity is controlled under mild conditions without the need of reducing agents. ${ }^{134}$ Iodogen also facilitates radioiodination reactions under mild conditions. In this case, iodogen is dissolved in organic solvents and evaporated, to fix the molecule on the walls of the reaction vessel, preventing dissolution in water and direct contact with the biomolecule/NP. All these iodine radiolabelling mediators are generally limited to the presence of tyrosine or histidine moieties in the surface of the nanomaterials. The Bolton-Hunter reagent, a radioiodination mediator based on a pre-radiolabelled $N$-hydroxysuccinimide group, is frequently used for the radiolabelling of nanomaterials with free amino groups on the surface - extending the flexibility of the nanomaterial radioiodination protocols (Fig. 16B). With advantages and drawbacks, these radiolabelling mediators have been applied to the radiolabelling with ${ }^{124} \mathrm{I},{ }^{125} \mathrm{I}$ or ${ }^{131} \mathrm{I}$ of a vast number of nanomaterials (Table 7). Generally, these protocols rendered high radiochemical yields; although, in some examples,

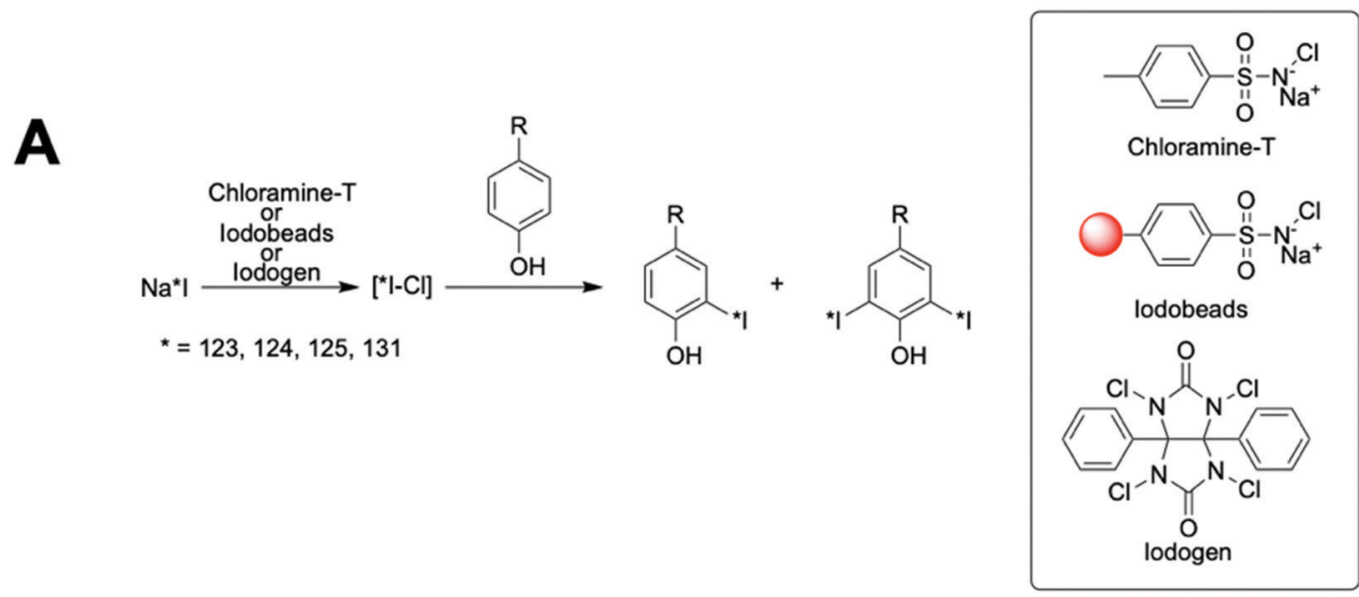

B
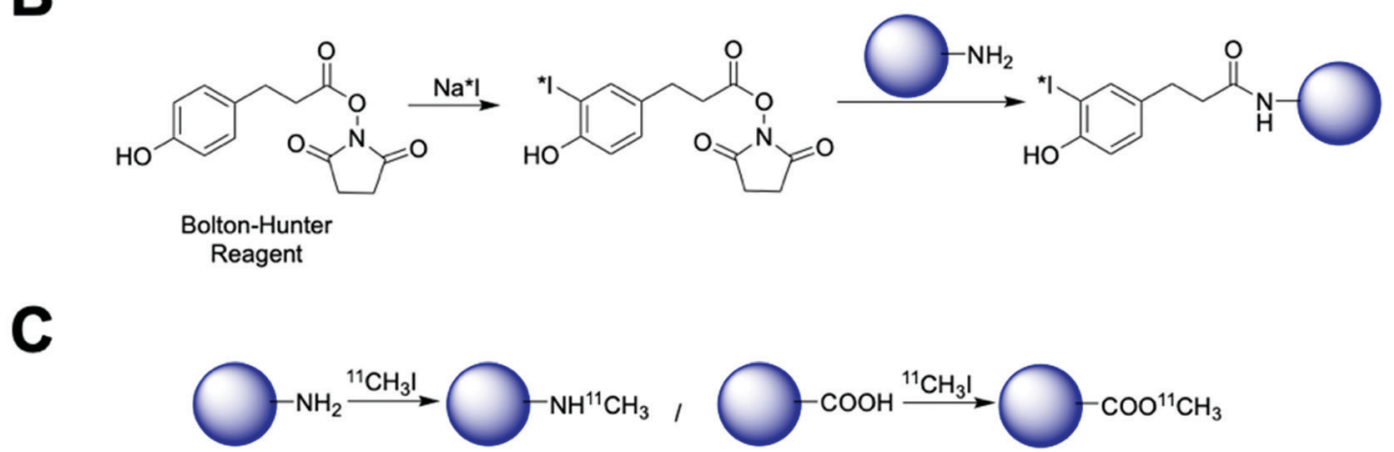

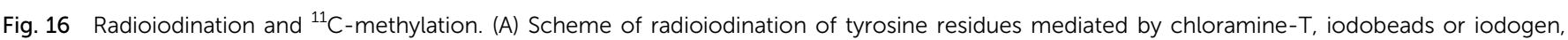

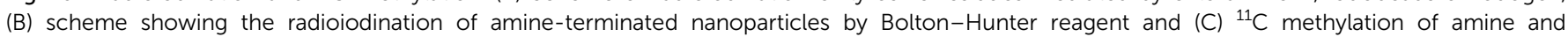
carboxylate-functionalised nanoparticles. 
Table 7 Radioiodinated nanomaterials by chloramine-T, iodobeads, iodogen and Bolton-Hunter reagent

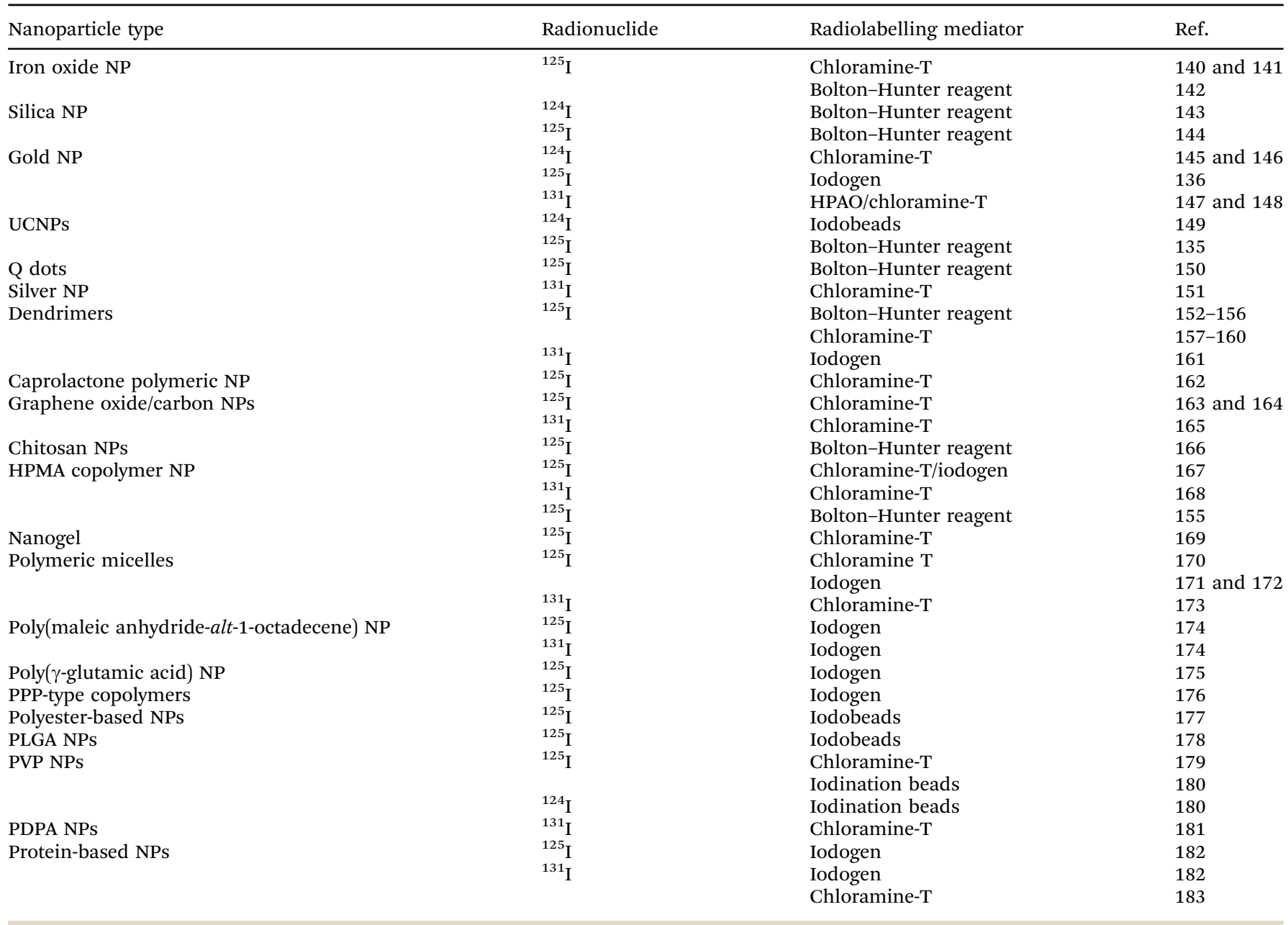

poor radiochemical stability were reported giving an undesirable accumulation in the thyroid glands due to the iodine detachment from the nanomaterial. ${ }^{135-137}$ This situation has been previously attributed to an enzymatic-driven cleavage of the $\mathrm{C}-\mathrm{I}$ bond in some molecules. ${ }^{138,139}$

Besides iodine radionuclides, chloramine-T has been also used as mediator for efficient radiolabelling of dendrimers and polymeric NPs with ${ }^{76} \mathrm{Br}$ providing RCYs greater than $95 \% .{ }^{184,185}$ Other radio-halogenation reactions such as traditional nucleophilic or electrophilic substitutions have also been applied to nanomaterials for iodine radiolabelling as well as for radio-fluorination. ${ }^{186-189}$ Interestingly, as with chelator based methods, click-chemistry or biorthogonal reactions (Fig. 8D) have also recently been explored for the radio-halogenation of nanomaterials. These reactions are frequently fast, specific to certain prosthetic groups and regioselective allowing rapid and controllable radio-halogenation with high yields and stabilities. ${ }^{190}$ With this purpose, chemoselective oxime formation, alkyne-nitrone, copper catalysed azide-alkyne and azide-DBCO cycloadditions have been used for ${ }^{18} \mathrm{~F},{ }^{123} \mathrm{I}$ and ${ }^{125} \mathrm{I}$ radiolabelling of both organic and inorganic nanomaterials. ${ }^{191-197}$ As a main drawback, biorthogonal reactions require the control over the synthesis, characterisation and reactivity of two independent species, complicating their potential clinical translation.

${ }^{11} \mathrm{C}$ methylation reactions can be also applied for the radiolabelling of nanomaterials (Fig. 16C). Sharma et al. reported the radiolabelling of iron oxide NPs using $\left[{ }^{11} \mathrm{C}\right] \mathrm{CH}_{3} \mathrm{I}$ as a precursor to conduct N- and O-methylation on the coating of the NPs with poor RCY, but high RCS. ${ }^{132}$ Although this study represented a good proof-of-concept, the very short half-life of the radionuclide (20.4 $\mathrm{min}$ ) does not seem to be suitable for biodistribution studies on nanomaterials that commonly show prolonged biological half-lives.

4.4.4 Heterogeneous/homogeneous radioisotopic exchange. Heterogeneous and homogeneous radioisotopic exchange are based on the replacement (or exchange) of stable elements present on nanomaterials with radionuclides (Fig. 17). The distinction between these two methods is whether the exchange occurs between different elements (heterogeneous exchange) or between different isotopes of the same element (homogeneous exchange).

A key advantage of this radiolabelling approach is its simplicity; however, few combinations of NP-radionuclide are truly effective with only a few examples in the literature of nanomaterials being radiolabelled by these methods. 

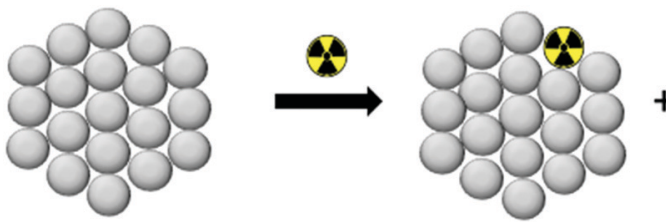

Fig. 17 Schematic representation of the radioisotopic exchange mechanism.

Homogeneous radioisotopic exchange between ${ }^{19} \mathrm{~F}$ and ${ }^{18} \mathrm{~F}$ has been reported as an attractive strategy for the radiolabelling of up-converting NPs (UCNPs). Two types of UCNPs with $\mathrm{NaYF}_{4}$ and $\mathrm{NaGdF}_{4}$ cores doped with $\mathrm{Yb}^{3+}$ and $\mathrm{Er}^{3+}$ have been investigated. $\mathrm{NaYF}_{4}$ (Fig. 18A) particles showed higher RCYs ( 92\%) than $\mathrm{NaGdF}_{4}$ with moderate radiochemical yields up to $43 \%$ when radiolabelling is conducted at room temperature for 1-10 min. Both formulations reported high RCPs (>95\%) with fast clearance from blood to liver and spleen for $\mathrm{NaYF}_{4}: \mathrm{Y}, \mathrm{Er}$ (Fig. 18B). Although the high bone accumulation (up to $12 \%$ ID per g, Fig. 18C) found during in vivo studies strongly suggest radiochemical instability $\left(\left[{ }^{18} \mathrm{~F}\right] \mathrm{F}\right.$-fluoride is known to accumulate in bone, Table 5) ${ }^{198-201}$ Heterogenous exchange has been used on the radiolabelling of iron oxide NPs (IONPs) with ${ }^{68} \mathrm{Ga}$, quantum dots (QDs) with ${ }^{68} \mathrm{Ga}$ and ${ }^{64} \mathrm{Cu}$ and UCNPs with ${ }^{153} \mathrm{Sm} .{ }^{202-205}$ The method provided radiolabelled NPs with high RCY and purity. The mild and fast radiolabelling conditions required for ${ }^{68} \mathrm{Ga}$-QDs $\left(37{ }^{\circ} \mathrm{C}\right.$ for $\left.15 \mathrm{~min}\right)$ or ${ }^{64} \mathrm{Cu}$-QDs $\left(60{ }^{\circ} \mathrm{C}\right.$ for $1 \mathrm{~h}$, Fig. 18D) suggest a facile heterogeneous exchange on QDs and therefore, an appropriate radiolabelling strategy.
In addition, in vivo PET biodistribution of ${ }^{64} \mathrm{Cu}-\mathrm{QDs}$ in tumourbearing mice revealed passive accumulation of the particles in the tumour by EPR effect with liver and spleen excretion (Fig. 18E).$^{204}$ As this biodistribution profile could be attributed to free ${ }^{64} \mathrm{Cu}^{2+}$, the authors further conducted ICP measurements on excised tissues after the injection of non-radioactive QDs. The results indicated a linear correlation between the ex vivo gamma counter quantification and the ICP measurements, confirming the ${ }^{64} \mathrm{Cu}$-QDs biodistribution of the PET imaging. On the other hand, the harsh conditions for ${ }^{68} \mathrm{Ga}-\mathrm{IONPs}$ and ${ }^{153} \mathrm{Sm}$-UCNPs $\left(T=100-300{ }^{\circ} \mathrm{C}\right.$ for $\left.1-4 \mathrm{~h}\right)$ suggest that milder radiolabelling strategies may be more appropriate, particularly if heating results in changes of the physicochemical properties of these NPs.

4.4.5 Chemical adsorption of radionuclides. In this method, the chemistry of the nanomaterial surface is leveraged to directly attach radionuclides. The majority of examples are based on the formation of coordination bonds between chemical groups on the nanomaterial surface such as $\mathrm{Fe}_{3} \mathrm{O}_{4}$, $-\mathrm{PO}_{3} \mathrm{H},-\mathrm{SH}$ or $-\mathrm{OH}$ and the radionuclide (Fig. 19), although other mechanisms are also possible.

This strategy, sometimes known as chemisorption, has been historically studied for other applications; mainly in catalysis and analytical chemistry to shed light on the mechanisms of interaction between metals and materials. ${ }^{206,207}$ Nevertheless, the first application for the radiolabelling of a nanomaterial appeared in 2013, where Cheng et al. described the chemical adsorption of various ${ }^{*}$ As $(*=71,72,74,76)$ radionuclides on the surface of a magnetite (IONP) NPs (Fig. 20A). ${ }^{208}$ In this case,
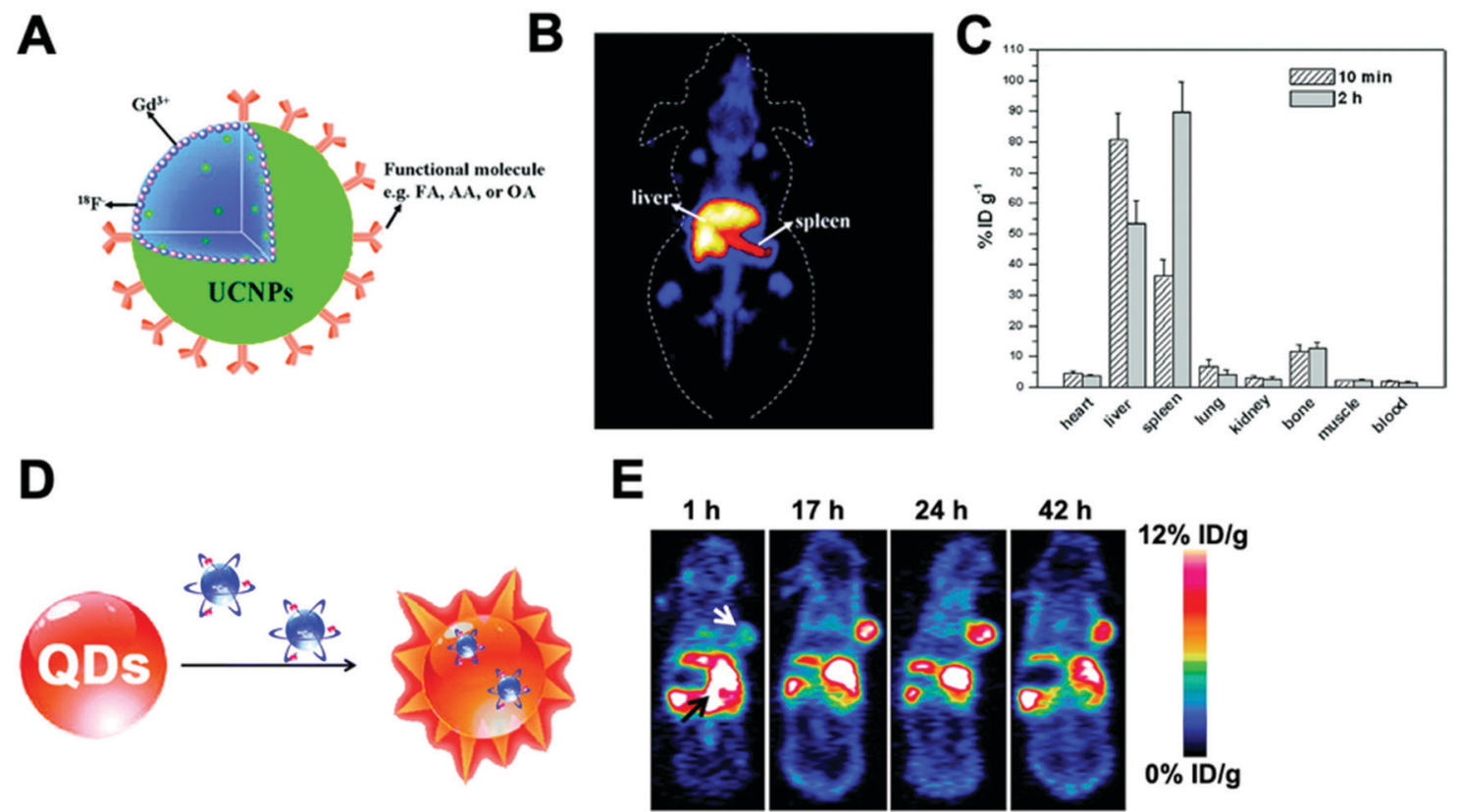

Fig. 18 (A) Schematic of fluorine-18-labeled magnetic-upconversion functional nanocrystals. FA: folic acid; OA: oleic acid; AA: aminocaproic acid, (B) Kunming mice PET imaging 10 min postinjection of ${ }^{18} \mathrm{~F}-\mathrm{AA}-\mathrm{Gd}-\mathrm{UCNPs}\left(200 \mu \mathrm{g} \mathrm{mL}{ }^{-1}\right)$, (C) biodistribution of ${ }^{18} \mathrm{~F}-\mathrm{AA}-\mathrm{Gd}-\mathrm{UCNPs}$ at $10 \mathrm{~min}$ and $2 \mathrm{~h}$ postinjection; the data shown are based on five mice per group, (D) design of self-illuminating ${ }^{64} \mathrm{Cu}$-doped QDs, (E) representative whole-body coronal PET images of U87MG tumour-bearing mice at $1,17,24$, and $42 \mathrm{~h}$ after intravenous injection of $250 \mu \mathrm{Ci}$ of ${ }^{64} \mathrm{Cu}$-doped QD580 ( $n=3$ ). White arrow, tumour area; black arrow, liver area. Adapted and reproduced with permission from ref. 198 and 204. 

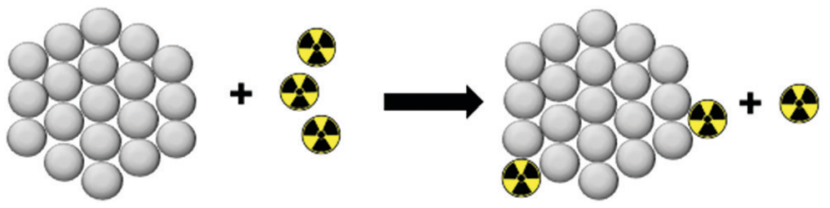

Fig. 19 Schematic representation of the chemical adsorption strategy.

the radiolabelling mechanism was attributed to the formation of stable As- $\mathrm{Fe}_{3} \mathrm{O}_{4}$ complexes where $\mathrm{As}^{\mathrm{III}} \mathrm{O}_{3}$ trigonal pyramids or $\mathrm{As}^{\mathrm{V}} \mathrm{O}_{4}$ tetrahedra may form on vacant tetrahedral spaces within the $\mathrm{Fe}_{3} \mathrm{O}_{4}$ octahedrally terminated (111) surface. The biodistribution of the ${ }^{*}$ As-IONPs was studied by PET imaging in Balb/C mice after i.v. injection of free ${ }^{*}$ As and ${ }^{*}$ As-IONPs. The images showed a renal elimination for the free ${ }^{*}$ As with high uptake in the bladder at $0.5 \mathrm{~h}$ and $3 \mathrm{~h}$ post-injection
(Fig. 20B). Elimination through liver and spleen was observed for the ${ }^{*}$ As-IONPs with significant signal in the bladder corresponding, most likely, to the in vivo desorption of *As from the NPs (Fig. 20C). After this work, several IONPs have been reported using the chemical adsorption strategy with a variety of other radionuclides. For example, feraheme/ferumoxytol NPs were successfully radiolabelled with different metallic radionuclides such as ${ }^{89} \mathrm{Zr},{ }^{64} \mathrm{Cu}$ and ${ }^{111} \mathrm{In}$ with radiochemical yields between $66-93 \%$ and radiochemical purities greater than $98 \%{ }^{209}$ The greater RCY $(93 \pm 3 \%)$ was obtained using either $\left[{ }^{89} \mathrm{Zr}\right] \mathrm{Zr}$-oxalate or $\left[{ }^{89} \mathrm{Zr}\right] \mathrm{ZrCl}_{4}$ at $\mathrm{pH}=8$ and $120{ }^{\circ} \mathrm{C}$ (Fig. 20D). With a RCS $>90 \%$ in human plasma, the biodistribution studies by PET/CT in wild-type B6C3F1/J mice revealed a circulation time in blood between 6-8 hours with final accumulation in liver, spleen and lymph nodes (high uptake in mesenteric lymph nodes) (Fig. 20E).

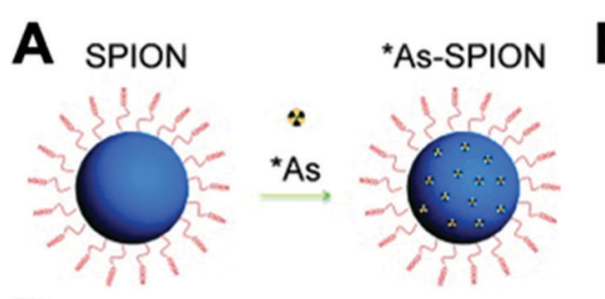

D

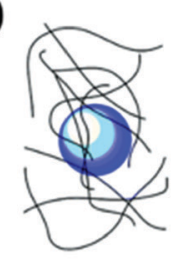

Feraheme

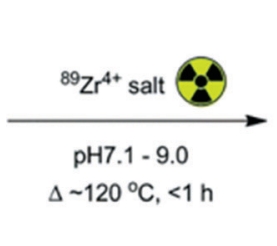

$$
\text { (1) }
$$
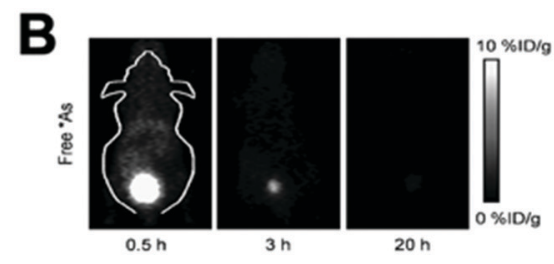

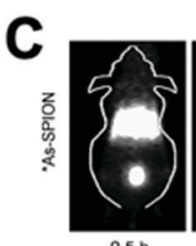

$0.5 \mathrm{~h}$

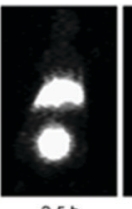

$2.5 \mathrm{~h}$
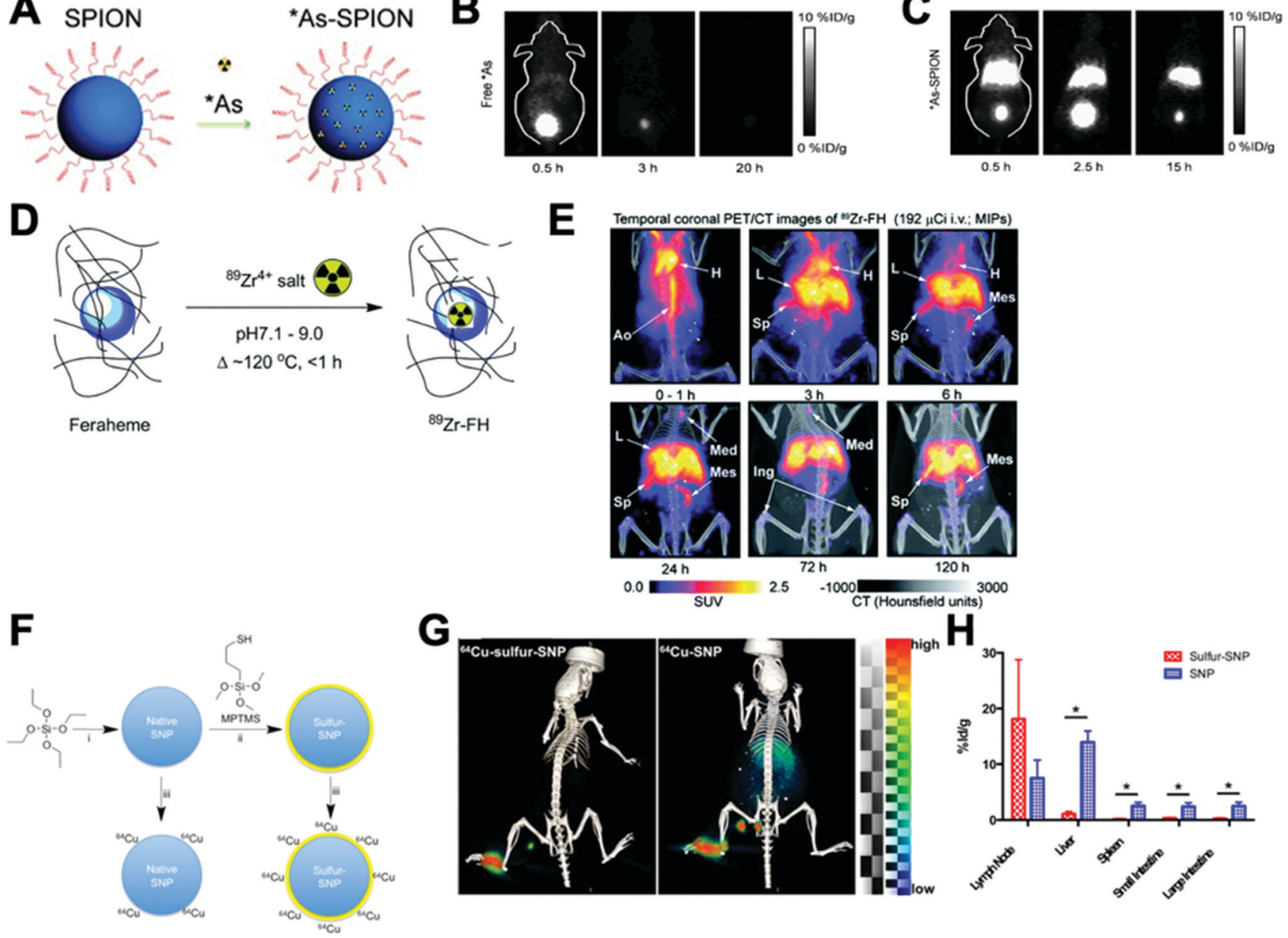

${ }^{89} \mathrm{Zr}-\mathrm{FH}$
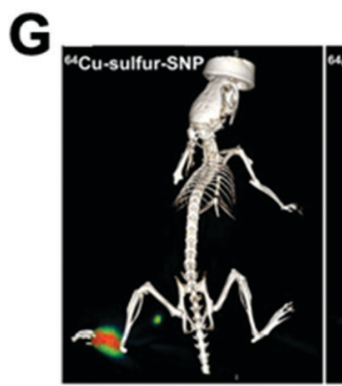

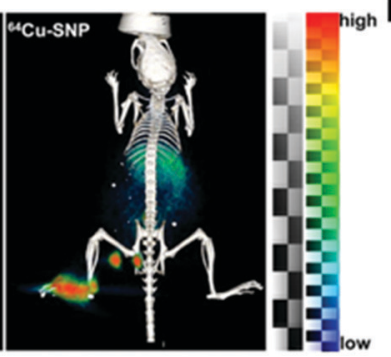

H

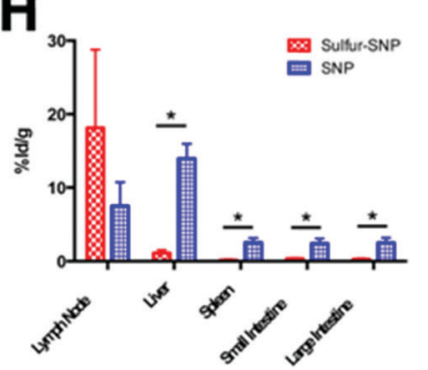

Fig. 20 (A) Chelator-free synthesis of *As-SPIONs, (B) serial in vivo PET images of free *As at different time points after intravenous injection into mice, (C) serial in vivo PET images of *As-SPION at different time points after intravenous injection into mice, (D) reaction of $\mathrm{FH}$ with ${ }^{89} \mathrm{Zr}{ }^{4+}$ ion salts (oxalate or chloride) to give radiolabeled ${ }^{89} \mathrm{Zr}-\mathrm{FH}$, (E) temporal PET/CT maximum intensity projection (MIP) images recorded between 0-120 h post-i.v. injection of ${ }^{89} \mathrm{Zr}-\mathrm{FH}$ in B6C3F1/J wild-type mice. Ao = aorta; $\mathrm{H}=$ heart; $\mathrm{L}=$ liver; $\mathrm{Sp}=$ spleen; Mes = mesenteric lymph node; Ing = inguinal lymph, (F) reaction schematic. Although native SNP (blue) stably bind hard oxophilic radiometals such as ${ }^{89} \mathrm{Zr}$ and ${ }^{68} \mathrm{Ga}$, thiol-functionalization (yellow) of SNP allows stable retention of soft, sulfur-avid copper-64. (G) PET/CT and biodistribution of ${ }^{64} \mathrm{Cu}$-sulfur-SNP and ${ }^{64} \mathrm{Cu}-\mathrm{SNP}$ injected into the footpad allow lymph node imaging with little systemic uptake at $14 \mathrm{~h}$ post-injection, $(\mathrm{H})$ quantitative ex vivo biodistribution values. Adapted and reproduced with permission from ref. 208-210. 
Additionally, silica NPs have showed particularly high affinities of oxophilic cations - such as ${ }^{68} \mathrm{Ga},{ }^{111} \mathrm{In},{ }^{177} \mathrm{Lu},{ }^{90} \mathrm{Y}$ and ${ }^{89} \mathrm{Zr}$ - towards the silanol groups on the NP surface (see Section 4.6.3). This allows simple, fast and robust radiolabelling of silica-based nanomaterials. ${ }^{211}$ However, this work described a poor RCS for the radiolabelling with ${ }^{64} \mathrm{Cu}$. To overcome this limitation, the authors reported the functionalisation of the silica NPs (SNP) to introduce thiol groups on the surface (sulfur-SNP) (Fig. 20F). ${ }^{212}$ This brief modification increases the RCS from $34.9 \pm 5.8 \%$ for SNP to $90.9 \pm 5.8 \%$ for the sulfur-SNP. These results were confirmed by in vivo PET/CT studies in athymic mice injected in the footpad (Fig. 20G). Whilst SNP showed accumulation in liver, spleen and intestines due to the free ${ }^{64} \mathrm{Cu}^{2+}$, sulfur-SNP were only observed in the footpad and draining lymph nodes as confirmed in the quantitative ex vivo biodistribution (Fig. $20 \mathrm{H}$ ).

A key drawback of the chemical adsorption strategy is the high temperatures required for the radiochemical reactions, that can be limiting for heat-sensitive NP formulations. Additionally, the strength of the chemical interaction between the radionuclide and the nanomaterial surface must be carefully considered to avoid radiochemical stability issues, such as those reported in the radiolabelling of $\mathrm{Fe}_{3} \mathrm{O}_{4} @ \mathrm{Al}(\mathrm{OH})_{3} \mathrm{NPs}$ with ${ }^{18} \mathrm{~F} .{ }^{213-215}$ In these studies, that relied on the formation of the theoretically strong $\mathrm{Al}-{ }^{18} \mathrm{~F}$ bonds, it was found that significant release of ${ }^{18} \mathrm{~F}$-fluoride occurred in vivo, as evident from the increasing high signal from bone reported by Cui et al. ${ }^{215}$

4.4.6 Physical interaction between materials and radionuclides. This method involves any mechanism where the radionuclide is physically attached to the nanomaterial, for example based on weak electrostatic interactions (physisorption) or driven by the presence of cavities, defects or grooves in the nanomaterial (Fig. 21).

Although plausible, this methodology has not been widely explored for the radiolabelling of nanomaterials with only few examples reported in the literature. A key example of this strategy reported the encapsulation of ${ }^{64} \mathrm{Cu}$ into the cavity of single-wall carbon nanotubes (SWCNTs). ${ }^{216}$ The radiolabelled SWCNTs presented quantitative RCP and high RCS in PBS after $24 \mathrm{~h}$ of incubation. However, the RCS decreased to $63 \%$ in $50 \%$ mouse serum confirming the poor stability of the radiolabelling. This is a good example on the application of the well-known loading capabilities of nanotubes to increase the specific molar activities of radionuclides in nanomaterials. Although the exploitation of physical properties of nanomaterials as the radiolabelling driven force is an interesting approach, it is currently not extensively used due to the hypothetical low radiochemical stability issues - as well as the lack of appropriate materials amenable to fully exploit these radiolabelling mechanisms.

\subsection{Radiolabelling of organic nanomaterials}

In the previous sections we have outlined the main methodologies of incorporating radionuclides into nanomaterials. We will now review the radiolabelling of specific types of nanomaterials, linking them with the different radiolabelling methods discussed above, and the potential benefits/ drawbacks of each approach. This section will focus on organicbased nanomaterials and will be followed by inorganic nanomaterials in Section 4.6.

4.5.1 Liposomes. Liposomes are spherical particles consisting of a phospholipid bilayer surrounding an aqueous core (Fig. 22A) and have been widely explored as in vivo drug delivery systems - also known as liposomal nanomedicines. In particular, PEGylated long circulating liposomes (LCLs or stealth liposomes) have arguably had the most significant impact in clinical medicine to date, particularly in the field of anticancer drug delivery - with several products clinically available. In the context of the NP radiolabelling field, liposomes have the largest proportion of examples in the literature with a huge diversity of radiolabelling methods available (Table 8). ${ }^{217}$ The different techniques employed to radiolabel liposomes will be described, with key examples of each discussed.

The direct attachment of radionuclides to the surface of liposomes - without the use of chelators - was first described by Richardson et al. who showed that liposomes can be directly labelled with ${ }^{99 \mathrm{~m}} \mathrm{Tc}$ after reduction of pertechnetate using stannous chloride $\left(\mathrm{SnCl}_{2}\right)$ as a reducing agent. ${ }^{221-225}$ To the best of our knowledge, the exact binding site of ${ }^{99 \mathrm{~m}} \mathrm{Tc}$ is not known; however, one likely possibility is chelation by the phosphonate groups on the liposome phospholipid surface. Labelling efficiencies (RCY) of $>97 \%$ could be achieved after $15 \mathrm{~min}$ at room temp. However, there have been reports of in vivo instability of the radiolabel using this method. ${ }^{329}$ This direct labelling approach was also used by Abou et al. with ${ }^{89} \mathrm{Zr}$. However, this interaction was shown to be weak, resulting in low serum and in vivo stability. ${ }^{226}$

Non-chelator labelling of liposomes has also been achieved with radiofluorine-based agents. Several groups used 3-[ $\left[{ }^{18}\right.$ F]fluoro-1,2-dipalmitoylglycerol $\left(\left[{ }^{18}\right.\right.$ F]FDP, Fig. 22B $),{ }^{218,227-229}$ which was added during liposomal preparation. Radiolabelled liposomes could be prepared in $c a .1 \mathrm{~h}$ with a RCY of $70 \%$.
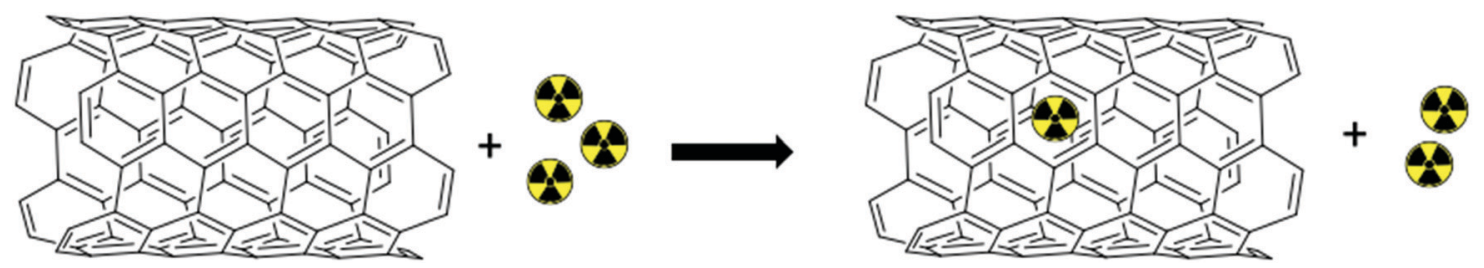

Fig. 21 Schematic representation of the radiolabelling strategy involving physical interaction between materials and radionuclides. 
A

Fig. 22 (A) Schematic representation of the different methods to radiolabel liposomes. Radionuclides can be bound to the surface using chelators or chelate-free or trapped intra-liposomally. (B) Chemical structures of $\left[{ }^{18} \mathrm{~F}\right] \mathrm{FDP}$ and $\left[{ }^{18} \mathrm{~F}\right] \mathrm{SteP} 2$. (left) PET image of $\left[{ }^{18} \mathrm{~F}\right] \mathrm{FDP}$ liposome in a rat model during a 90 min scan (right). Adapted with permission from Marik et al. ${ }^{218}$ (C) Schematic for the liposomal-labelling method with ${ }^{89} \mathrm{Zr}$, with different PEG chain lengths between the DFO chelator and liposome surface, used by Seo et al. (D) PET images at indicated time of ${ }^{89} \mathrm{Zr}$ liposomes in mammary tumour bearing mice with no PEG chain (top), a 1k PEG chain (middle), and a 2k PEG chain (bottom) between the DFO chelator and liposomal surface. Clear differences in tumour and liver uptake can be observed. Adapted with permission from Seo et al. ${ }^{219}$ (E) Gamma camera images of ${ }^{99 m} \mathrm{Tc}$-labeled HYNIC liposomes (top row) and ${ }^{99 \mathrm{~m}} \mathrm{Tc}$-labelled HMPAO liposomes (bottom row) in rats with S. aureus abscess in calf muscle. Adapted from Laverman et al. ${ }^{220}$

In vivo stability was shown with no observable bone uptake (a consequence of defluorination). ${ }^{218}$ Alternatively, Urakami et al. synthesised an amphiphilic probe, 1- $\left[{ }^{18} \mathrm{~F}\right]$ fluoro-3,6-dioxatetracosane $\left(\left[{ }^{18} \mathrm{~F}\right] \mathrm{SteP} 2\right.$, Fig. 22B). ${ }^{231-234}$ The long alkyl chain on the probe allowed intercalation with the lipid bilayer on the liposome surface allowing a LE and stability in serum (after $30 \mathrm{~min}$ ) of $>80 \%$. $^{232}$

Chelator-based radiolabelling of liposomes is primarily performed by the attachment of a chelator onto the liposome surface, either to the phospholipid or PEG chains present on LCLs (Fig. 22A). Liposomes pre-formulated with DTPA conjugated to the phospholipid on the liposome have been widely used with several different radioisotopes; particularly with ${ }^{99 \mathrm{~m}} \mathrm{Tc}$ - however, low serum and in vivo stability was observed using this method. ${ }^{238-240}$ Several reports have also shown that DTPA functionalised liposomes allow $>95 \%$ RCY with ${ }^{111}$ In under mild conditions $\left(25-37{ }^{\circ} \mathrm{C}\right.$, up to $\left.1 \mathrm{~h}\right) .^{244,249-255}$ Interestingly, Helbok et al. reported a direct comparison of the radiolabelling of DTPA-functionalised PEGylated liposomes with several different radionuclides. ${ }^{244}$ The liposomes were labelled with ${ }^{99 \mathrm{~m}_{\mathrm{Tc}}}$ (using both $\left[{ }^{99 \mathrm{~m}} \mathrm{Tc}\right]\left[\mathrm{TcO}_{4}\right]^{-}$and $\left[{ }^{99 \mathrm{~m}} \mathrm{Tc}\right]\left[\mathrm{Tc}(\mathrm{CO})_{3}\right]^{+}$and $\left.{ }^{111} \mathrm{In}\right)$, with the latter showing the most favourable labelling 
Table 8 Table summarising the methods, radioisotopes and techniques employed to radiolabel liposomes

\begin{tabular}{|c|c|c|c|}
\hline Radiolabelling method & Radioisotope & Radiolabelling mediator & Ref. \\
\hline Surface non-chelator & $\begin{array}{l}{ }^{99} \mathrm{~m}_{\mathrm{Tc}} \\
{ }^{89} \mathrm{Zr} \\
{ }^{18} \mathrm{~F}\end{array}$ & $\begin{array}{l}\text { Direct labelling via } \mathrm{SnCl}_{2} \text { reduction } \\
\text { Chelate free } \\
{\left[{ }^{18} \mathrm{~F}\right] \text { FDP }} \\
\left.{ }^{18} \mathrm{~F}\right] \text {-Fluorocholesteryl ether } \\
\left.{ }^{18} \mathrm{~F}\right] \text { SteP2 } \\
\text { CuAAC click reaction }\end{array}$ & $\begin{array}{l}221-225 \\
226 \\
218 \text { and } 227-229 \\
230 \\
231-234 \\
235 \text { and } 236\end{array}$ \\
\hline Surface chelator-based & $\begin{array}{l}{ }^{52} \mathrm{Mn} \\
{ }^{177} \mathrm{Lu} \\
{ }^{90} \mathrm{Y} \\
{ }^{166} \mathrm{Ho} \\
{ }^{89} \mathrm{Zr}\end{array}$ & $\begin{array}{l}\text { DTPA-sterylamine } \\
\text { DTPA } \\
\text { DTPA via }{ }^{99 m} \text { Tc-tricarbonyl } \\
\text { HYNIC + tricine co-ligand } \\
\text { 2-Iminothiolane via }{ }^{99 m_{1}} \text { Tc-tricarbonyl } \\
\text { DTPA-sterylamine } \\
\text { DTPA } \\
\text { DTPA } \\
\text { NODAGA } \\
\text { BAT } \\
\text { TETA } \\
\text { CB-TE2A } \\
\text { DOTA } \\
\text { DO3A } \\
\text { DO3A } \\
\text { DTPA } \\
\text { DTPA } \\
\text { DTPA } \\
\text { DFO }\end{array}$ & $\begin{array}{l}237 \\
238-244 \\
244 \\
220 \text { and } 245-247 \\
248 \\
237 \\
244 \text { and } 249-255 \\
244 \\
256 \\
257-261 \\
262 \text { and } 263 \\
262 \\
264 \\
265-267 \\
267 \\
244 \\
268 \\
269 \\
219 \text { and } 270-274\end{array}$ \\
\hline Complex trapping & $\begin{array}{l}{ }^{99 m} \mathrm{Tc} \\
{ }^{111} \mathrm{In} \\
{ }^{159} \mathrm{Gd} \\
{ }^{225} \mathrm{Ac}\end{array}$ & $\begin{array}{l}\text { DTPA complex during formulation } \\
\text { DTPA complex during formulation } \\
\text { DTPA complex during formulation } \\
\text { DOTA complex during formulation }\end{array}$ & $\begin{array}{l}275-278 \\
279 \\
280 \\
281\end{array}$ \\
\hline Ionophore-based (chelator binding) & $\begin{array}{l}{ }^{90} \mathrm{Y} \\
{ }^{67} \mathrm{Ga} \\
{ }^{177} \mathrm{Lu} \\
{ }^{64} \mathrm{Cu} \\
{ }^{52} \mathrm{Mn}\end{array}$ & $\begin{array}{l}\text { A23187 (NTA) } \\
\text { Oxine (NTA) } \\
\text { Acetylacetone (NTA) } \\
\text { Tropolone (NTA) } \\
\text { Oxine (DFO) } \\
\text { Oxine (DTPA) } \\
\text { A23187 (DTPA) } \\
\text { Oxine (DFO) } \\
\text { Tropolone (DFO) } \\
\text { Oxine (DTPA) } \\
\text { 2-HQ (DOTA) } \\
\text { Oxine (DOTA) } \\
\text { 8HQ-2Cl (DOTA) } \\
\text { 8HQ-2I (DOTA) } \\
\text { Oxine (DOTA) } \\
\text { A23187 (DOTA) } \\
\text { Oxine (DFO) } \\
\text { 2HQ (DFO) }\end{array}$ & $\begin{array}{l}282 \text { and } 283 \\
284 \text { and } 285 \\
286 \\
287 \\
288 \text { and } 289 \\
254,279 \text { and } 290-293 \\
294 \\
295 \text { and } 296 \\
295 \text { and } 296 \\
297 \\
298-300 \\
267 \\
301 \text { and } 302 \\
303\end{array}$ \\
\hline Ionophore based (drug binding) & $\begin{array}{l}{ }^{89} \mathrm{Zr} \\
{ }^{52} \mathrm{Mn} \\
{ }^{64} \mathrm{Cu} \\
{ }^{111} \mathrm{In}\end{array}$ & $\begin{array}{l}\text { Oxine } \\
\text { Oxine } \\
2 \mathrm{HQ} \\
\text { Oxine }\end{array}$ & $\begin{array}{l}304 \text { and } 305 \\
304 \text { and } 306 \\
304 \\
307\end{array}$ \\
\hline Unassisted loading (chelator binding) & ${ }^{64} \mathrm{Cu}$ & DOTA & $308-311$ \\
\hline Remote loading & $\begin{array}{l}{ }^{186} \mathrm{Re} \\
{ }^{188} \mathrm{Re} \\
{ }^{64} \mathrm{Cu} \\
{ }^{124} \mathrm{I} \\
{ }^{125} \mathrm{I}\end{array}$ & $\begin{array}{l}\text { HMPAO } \\
\text { DISIDA } \\
\text { BMEDA } \\
\text { BMEDA } \\
\text { BMEDA } \\
\text { 4-DEAP-ATSC } \\
\text { Amino diatrizoic acid } \\
\text { Amino diatrizoic acid }\end{array}$ & $\begin{array}{l}220,312 \text { and } 313 \\
314 \\
315-317 \\
318 \text { and } 319 \\
320-323 \\
324-327 \\
328\end{array}$ \\
\hline
\end{tabular}

( $>95 \%$ LE). Labelling with $\left[{ }^{99 \mathrm{~m}} \mathrm{Tc}\right]\left[\mathrm{TcO}_{4}\right]^{-}$was consistently lower (75\%), and $>80 \%$ RCY was achievable using $\left[{ }^{99 \mathrm{~m}_{\mathrm{Tc}}}\right]\left[\mathrm{Tc}(\mathrm{CO})_{3}\right]^{+}$ but only with 50 -fold more liposomes. The authors also demonstrated radiolabelling with ${ }^{68} \mathrm{Ga}$ and the therapeutic isotope 
${ }^{177} \mathrm{Lu}$ using the same formulation; achieving $>95 \%$ and $>80 \%$ RCY respectively - however higher concentrations of liposomes were necessary for the latter. ${ }^{244}$

A key consideration when radiolabelling liposomes via the surface is the biodistribution of these radiolabelled phospholipids in vivo, which may occur after tissue uptake/destruction of the liposomes. This was explored by Seo et al. who synthesised liposomes functionalised with the ${ }^{64} \mathrm{Cu}$-specific chelator TETA (Fig. 6). ${ }^{257-261}$ This allowed $>80 \%$ LE after $1 \mathrm{~h}$ at room temp, with $>90 \%$ stability in mouse serum for $48 \mathrm{~h}$. Interestingly, the ex vivo biodistribution at $48 \mathrm{~h}$ of the liposomes compared to the ${ }^{64} \mathrm{Cu}-\mathrm{PEG}-\mathrm{lipid}$, showed liver uptake of the latter was roughly 3-fold higher than the liposomes. ${ }^{257}$ This uptake of lipids, that may arise as a result of in vivo liposome decomposition, should be carefully considered when tracking liposomes, as it may lead to misinterpretation of the amount of liposomes present in the liver.

Furthermore, several reports have shown that the biodistribution of radiolabelled liposomes can easily be altered solely based on the position of the radiocomplex, which could be viewed as a drawback to surface labelling of liposomes. Seo and collaborators looked at labelling using ${ }^{64} \mathrm{Cu}$ complexes of 1,4,8,11-tetraazacyclotetradecane-1,4,8,11-tetraacetic acid (TETA) and 4,11-bis(carboxymethyl)-1,4,8,11-tetraazabicyclo(6.6.2)hexadecane (CB-TE2A, Fig. 6). ${ }^{262}$ Intriguingly, the authors showed that attaching the complex to either PEG or nonPEGylated lipids altered the biodistribution, with 5\% higher hepato-splenic uptake occurring after $48 \mathrm{~h}^{262}$ This work was later expanded by Seo et al. who performed surface labelling with ${ }^{89} \mathrm{Zr}$ using desferrioxamine (DFO) as a chelator, which allows radiolabelling at neutral $\mathrm{pH}$ with only mild heating. ${ }^{219,270-274}$ The authors compared the effect of increasing PEG-length between the liposomal surface and the ${ }^{89} \mathrm{Zr}$-DFO complex. $^{219}$ Three formulations were prepared with DFO attached directly to the lipid or with a $1 \mathrm{k}$ or $2 \mathrm{k}$ PEG spacer (Fig. 22C). No significant differences in terms of \% RCY, stability or blood half-life were observed. However, image-based analysis showed significantly higher tumour, liver and spleen uptake when using a 2k PEG spacer, over $7 \mathrm{~d}$ compared to the other two formulations (Fig. 22D). This highlights how small modifications in chelator position on the surface of radiolabelled liposomes can affect their biodistribution and pharmacokinetics.

Due to these potential drawbacks of chelator-based surface labelling, radiolabelling of liposomes is sometimes performed within the liposomal core. This approach can, in theory, increase the stability of the radiolabel as it is no longer present on the surface where it can interact with chelating compounds (e.g. serum proteins). However, the radiolabelling procedure can often become more complex; often involving the prior synthesis of a radiotracer to incorporate radionuclides inside the liposomes (see Section 4.3). Some of the earliest studies achieved this by simply encapsulating a radiometal complex with DTPA inside the liposomal core during formation of the liposomes (see Section 4.2.2). This was first done with ${ }^{99 \mathrm{~m}} \mathrm{Tc},{ }^{275-278}$ and later with ${ }^{111} \mathrm{In}^{279}$ and ${ }^{159} \mathrm{Gd}-\mathrm{DTPA},{ }^{280}$ as well as encapsulating the DOTA complex of ${ }^{225} \mathrm{Ac} .{ }^{281}$ One drawback of this method is the longer, more complicated radiosynthesis needed (especially relevant when using short-lived isotopes).

The most widely used 'intra-liposomal' radiolabelling method is the use of ionophores to transport radiometals across the lipid bilayer to encapsulated chelators (Fig. 10A and Section 4.3.1). The first example of this was reported by Gamble and collaborators who used the calcium ionophore A23187 (Fig. 11A) to transport ${ }^{111}$ In inside the liposomal core where it was chelated by encapsulated nitrilotriacetic acid (NTA, Fig. 6) allowing $>90 \%$ RCY..$^{282,283}$ Since then, several different ionophore and encapsulated chelator combinations have been reported (Table 8). A key study by Harrington and collaborators reported using the ionophore 8-hydroxyquinoline (oxine; Fig. 11A) to radiolabel liposomes containing DTPA with ${ }^{111} \mathrm{In}$, which allowed $>90 \%$ LE after $15 \mathrm{~min}$ incubation and high serum stability for up to 10 days. $^{290-292}$ An important study by Van der Geest et al. compared this ionophore-based radiolabelling with chelator-based surface labelling with ${ }^{111}$ In using DTPA-DSPE liposomes - along with the labelling of empty liposomes (without DTPA). ${ }^{254}$ Labelling efficiencies and serum stability (after $48 \mathrm{~h}$ ) of $>95 \%$ were reported using both radiolabelling methods, whereas the empty liposomes showed lower LE $(62 \%)$ and serum stability (68\%). Interestingly, when comparing the in vivo distribution of the formulations in mice, the surface-labelled liposomes showed significantly higher liver uptake over $72 \mathrm{~h}$ - compared to the oxine-DTPA liposomes. ${ }^{254}$ This may indicate that release of $\left[{ }^{111} \mathrm{In}\right] \mathrm{In}-\mathrm{DTPA}-\mathrm{DSPE}$ from the liposomes is occurring, suggesting lower in vivo stability, as $\left[{ }^{111} \mathrm{In}\right] \mathrm{In}$-DTPA is rapidly cleared, ${ }^{291}$ whereas $\left[{ }^{111} \mathrm{In}\right] \mathrm{In}-\mathrm{DTPA}-$ DPSE (released from liposomes during degradation) will likely accumulate in the liver (vida supra).

A key consideration when using ionophore-based methods is the intra-liposomal $\mathrm{pH}$; which can affect the rate of radiometal release, and subsequent transchelation. Petersen et al. used the ionophore 2-hydroxyquinoline (2HQ, Fig. 11A) to radiolabel DOTA-encapsulated liposomes with ${ }^{64} \mathrm{Cu}$, which had different intra-liposomal pHs. ${ }^{298-300}$ Liposome loading was $>95 \%$ and $70 \%$ for $\mathrm{pH} 4$ and 5.9 respectively, suggesting the complexation by DOTA was affected. ${ }^{298}$ This concept was explored further by Jensen et al. who used several oxine derivatives to load ${ }^{52} \mathrm{Mn}$ into DOTA encapsulated liposomes. ${ }^{267}$ Labelling efficiencies above 90\% could be achieved with an intraliposomal of pH 4 when using oxine and 5,7-dichloro-8hydroxyquinoline (8HQ-2Cl, Fig. 11A), but increasing the $\mathrm{pH}$ to 7.8 led to a large reduction in labelling using oxine ( $c$. 30-70\% LE) whereas this was not observed for $8 \mathrm{HQ}-2 \mathrm{Cl}$. Therefore, the internal $\mathrm{pH}$ will not only affect the chelation by the internalised ligand, but also the dissociation of the ionophore complex used.

Our group showed that radiolabelling of liposomes is possible without the need for incorporated chelators and therefore without having to chemically modify the formulation. ${ }^{304-307}$ This is based on the metal-chelating properties of certain drug molecules (Fig. 23A and B), that are present in high concentrations inside the liposome, and able to bind the radionuclide after ionophoremediated transport across the lipid bilayer (Fig. 23A). For example, manganese complexes of doxorubicin via hydroxyl 
A
B

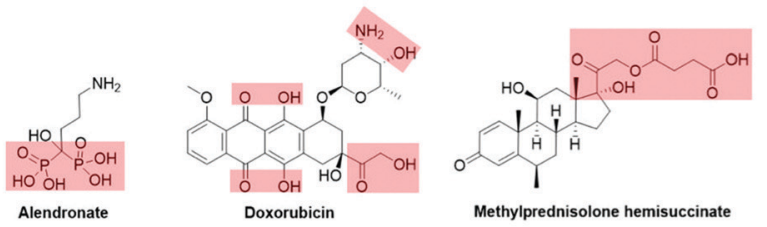

lonophore Drug with chelating

properties

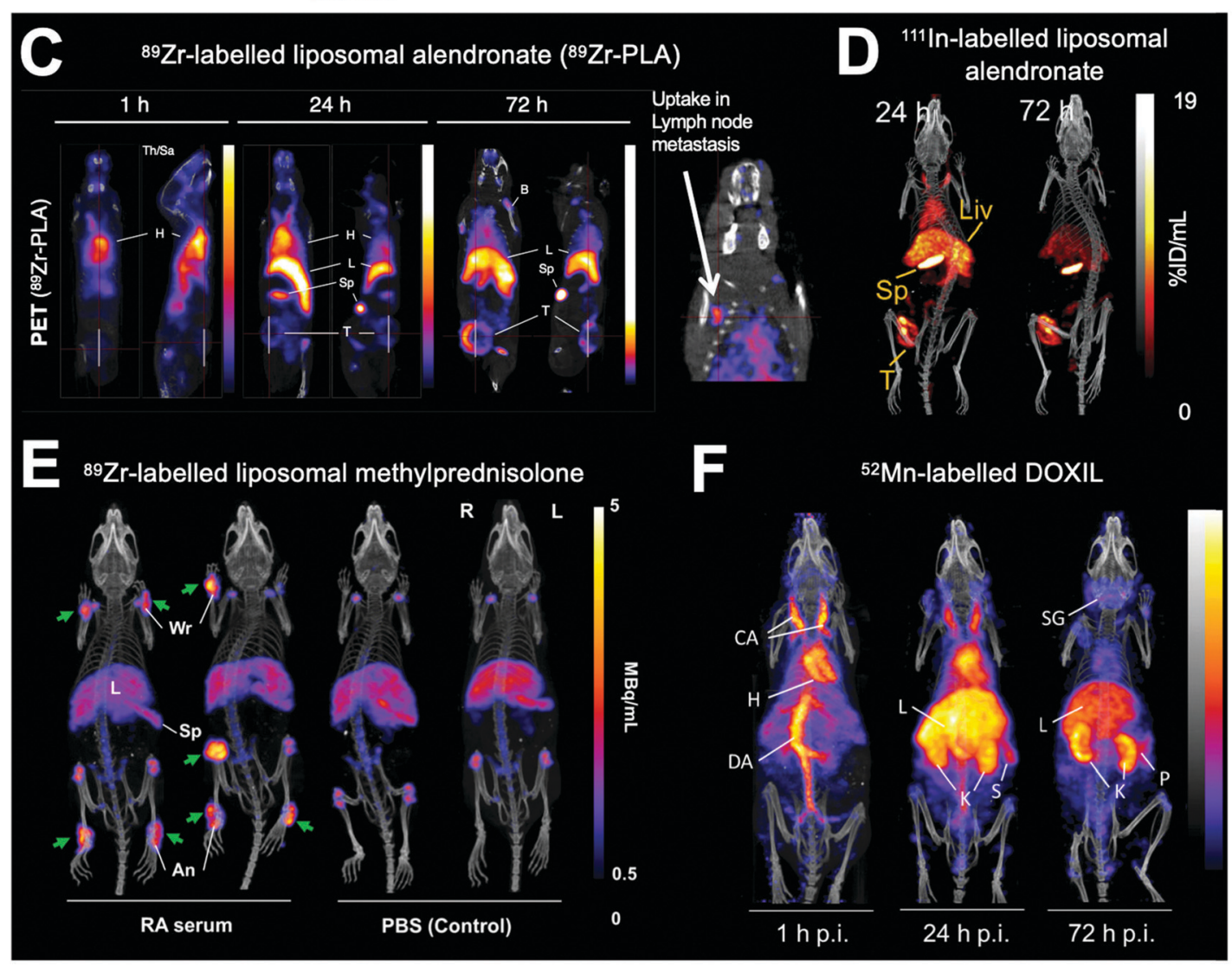

Fig. 23 (A) Schematic showing the ionophore-based method for radiolabelling liposomes using the chelating properties of drugs. (B) Chemical structures of drugs incorporated inside liposomes capable of chelating radiometals. (C) PET/CT images of PEGylated liposomal alendronate (PLA) labelled with ${ }^{89} \mathrm{Zr}$ in a mouse model of metastatic breast cancer, showing long circulation and gradual uptake in primary tumour (T) and lymph node metastasis. Adapted from Edmonds et al. ${ }^{304}$ (D) SPECT-CT images of ${ }^{111}$ In-labelled PEGylated liposomal alendronate in a breast cancer model. Adapted from Man et al. ${ }^{307}$ (E) PET/CT images of PEGylated liposomal methylpredinisolone hemisucinate labelled with ${ }^{89} \mathrm{Zr}$ in a model of arthritis (left) and control animals (right). High uptake in arthritic joints denoted by green arrows. Adapted with permission from Gawne et al. ${ }^{305}$ (F) PET/CT images in healthy mice of DOXIL radiolabelled with ${ }^{52} \mathrm{Mn}$. Images at $72 \mathrm{~h}$ show release and redistribution of ${ }^{52} \mathrm{Mn}$ from liposomes after tissue uptake. Adapted with permission from Gawne et $a^{306}$

and carbonyl groups on the doxorubicin backbone have been previously reported, ${ }^{330,331}$ and IR spectroscopy showed that $\mathrm{Zr}^{4+}$ interacted with the carboxylate present on methylprednisolone hemisuccinate. ${ }^{305}$ These interactions allowed us to radiolabel a variety of liposomal nanomedicines with ${ }^{111} \mathrm{In},{ }^{64} \mathrm{Cu},{ }^{89} \mathrm{Zr}$ and ${ }^{52} \mathrm{Mn}$ and image them longitudinally (Fig. 23C-F). ${ }^{304-307}$ This method overcomes the need to incorporate liposomes with a chelator which may limit its use to validate pre-formulated, commercially available liposomal nanomedicines. However, the extent of radiolabelling using this method will always be limited by the strength of interaction between the radiometal and the drug inside the liposomal formulation. ${ }^{304,305}$ Furthermore, the lack of a stable chelator means that release of the 'free radiometal' can occur after destruction of the liposomes. In particular, radioactive isotopes of endogenous metals, such as ${ }^{52} \mathrm{Mn}$ and ${ }^{64} \mathrm{Cu}$, may be more susceptible to trafficking out of the tissues and into the bloodstream, resulting in secondary uptake in other organs (Fig. 23F). Specifically, in the case of ${ }^{64} \mathrm{Cu}$ and ${ }^{52} \mathrm{Mn}$ it may be difficult to distinguish between 'free radiometal' uptake from that of liposomal uptake in the liver and even in tumours. ${ }^{332,333}$ This has been shown to be less of an issue when labelling with ${ }^{89} \mathrm{Zr}$ (a non-endogenous metal), 
which almost exclusively shows uptake in the bone (Fig. 23C and E). ${ }^{334}$

Interestingly, Henriksen et al. showed that use of an ionophore to transport radiometals across the lipid bilayer of liposomes is not always necessary. They found that by incubating unchelated ${ }^{64} \mathrm{Cu}^{2+}$ with liposomes containing a DOTA chelator allowed $>90 \%$ RCY after $30-60 \mathrm{~min}$ at $55{ }^{\circ} \mathrm{C} .{ }^{308-311}$ This 'unassisted loading' of ${ }^{64} \mathrm{Cu}$ was proposed to occur due to the formation of a steep copper gradient, across the lipid membrane, by the chelation of non-radioactive copper inside the liposomal core by the DOTA chelator. This gradient then causes diffusion of ${ }^{64} \mathrm{Cu}^{2+}$ into the liposome where it is trapped by chelation by the DOTA ligand. The increased simplicity of this technique is clearly beneficial, and additionally removes the need for ionophores, which are known to have a variety of biological activities. ${ }^{335}$ However, it may not be applicable to other radionuclides and more studies are required to fully understand the exact mechanism that allows charged hydrophilic ions such as $\mathrm{Cu}^{2+}$ to cross lipid bilayers.

The radiolabelling of liposomes can also be achieved by the remote loading of radiopharmaceuticals into the liposomal core (Section 4.3.2). Generally speaking, a neutrally charged, lipophilic, radiopharmaceutical crosses the lipid bilayer of liposomes into the aqueous core where it becomes protonated and trapped as a more hydrophilic form (Fig. 10B and Section 4.3.2). An early example of this was reported by Rudolph and collaborators who used ${ }^{99 \mathrm{~m}} \mathrm{Tc}$-labelled hexamethylpropyleneamine oxime (HMPAO, Fig. 11B) to radiolabel liposomes encapsulating with glutathione (GSH) which was necessary to allow high labelling of the liposomes $\left(>90 \%\right.$ LE). ${ }^{220,312,313}$ The authors postulated the complex would undergo reduction by interaction with glutathione, allowing trapping of the agent. Cao et al. also reported the GSH-dependent trapping in liposomes of the ${ }^{99 \mathrm{~m}} \mathrm{Tc}$ complex of diisopropyl iminodiacetic acid (DISIDA, Fig. 11B). ${ }^{314}$ Laverman et al. later compared remote loading of $\left[{ }^{99 \mathrm{~m}} \mathrm{Tc}\right] \mathrm{Tc}-\mathrm{HMPAO}$ with surface radiolabelling using HYNIC conjugated to the lipid bilayer of liposomes. ${ }^{20}$ No difference in serum stability after $48 \mathrm{~h}$, using the two methods was reported. However, in vivo tracking of the labelled liposomes. showed that kidney uptake was 3 -fold higher after $24 \mathrm{~h}$ for HMPAO-labelled liposomes, suggesting release of $\left[{ }^{99 \mathrm{~m}} \mathrm{Tc}\right] \mathrm{Tc}-\mathrm{HMPAO}$ from the liposomes (Fig. 22E). A similar method was described by Bao and collaborators using the chelator $\quad N, N$-bis(2-mercaptoethyl)- $N^{\prime}, N^{\prime}$-diethyl-ethylenediamine (BMEDA, Fig. 11B) for the remote-loading of liposomes with ${ }^{99 \mathrm{~m}} \mathrm{Tc},{ }^{315-317}{ }^{186} \mathrm{Re},{ }^{318,319}$ and ${ }^{188} \mathrm{Re}^{320-323}$ Labelling efficiencies with the ${ }^{99 \mathrm{~m}} \mathrm{Tc}$ complex were $c a .37 \% \mathrm{LE}$; with the presence of glutathione within the liposomal core allowing increased stability $(>80 \%)$ in serum over $72 \mathrm{~h}$ compared with empty liposomes $\left(<35 \%\right.$ stability).$^{315}$ However, in both of these examples, the need to encapsulate glutathione within liposomes to facilitate radiolabelling is a potential drawback, compared with other methods avoiding the need for modifications.

An excellent method by Lee $e$ al . reported a ${ }^{64} \mathrm{Cu}$ complex of diacetyl $\quad 4,4^{\prime}$-bis(3-(N,N-diethylamino)propyl)thiosemicarbazone (4-DEAP-ATSC, Fig. 11B) for the remote loading of liposomal nanomedicines without modification. ${ }^{324-327}\left[{ }^{64} \mathrm{Cu}\right][\mathrm{Cu}(4$-DEAPATSC)] allowed $>90 \%$ LE of several liposomal formulations after $10 \mathrm{~min}$ at $65{ }^{\circ} \mathrm{C}$. The radiolabelled liposomes showed high serum stability $>99 \%$ after $48 \mathrm{~h}$. The authors compared the ex vivo biodistribution of the radiolabelled liposomes with the $\left[{ }^{64} \mathrm{Cu}\right]\left[\mathrm{Cu}(4\right.$-DEAP-ATSC) $]$ complex and free ${ }^{64} \mathrm{Cu}^{2+}$ and showed that the liposomes and complex had similar uptake in the liver as well as that $\left[{ }^{64} \mathrm{Cu}\right]\left[\mathrm{Cu}(4\right.$-DEAP-ATSC) $]$ and 'free ${ }^{64} \mathrm{Cu}$ ' had similar pharmacokinetics. Copper-bisthiosemicarbazone complexes are not stable in vivo, ${ }^{336}$ and thus any $\left[{ }^{64} \mathrm{Cu}\right][\mathrm{Cu}(4$-DEAPATSC)] released from the liposomes will likely decompose and release free ${ }^{64} \mathrm{Cu}$ leading to accumulation in the liver ${ }^{64} \mathrm{Cu}$ in its free form. Furthermore, small amounts ( $c a .3 \%$ ID per g, $24 \mathrm{~h}$ p.i.) of tumour uptake of $\left[{ }^{64} \mathrm{Cu}\right][\mathrm{Cu}(4$-DEAP-ATSC) $]$ was observed, ${ }^{324}$ which matches previous observations that ${ }^{64} \mathrm{Cu}$ and its bisthiosemicarbazone complexes are known to accumulate in tumours. ${ }^{336,337}$ Hence, the release of the $\left[{ }^{64} \mathrm{Cu}\right][\mathrm{Cu}(4-$ DEAP-ATSC)] complex - and indeed other remote loading complexes - from liposomes is a key consideration when using this method as it may distort tumour and liver uptake values of the radiolabelled liposomes. Finally, Engudar et al. reported a novel radioiodinated compound, amino diatrizoic acid (ADA, Fig. 11B), for the remote loading into liposomes. ${ }^{328}\left[{ }^{125} \mathrm{I}\right]$-ADA and $\left[{ }^{124} \mathrm{I}\right]-\mathrm{ADA}$ could be incorporated into liposomes with $>70 \%$ LE after $2 \mathrm{~h}$. Good stability of the radiolabel in vivo was demonstrated by just $1 \%$ ID per $\mathrm{g}$ of the radioactivity in thyroid present after $72 \mathrm{~h}$.

4.5.2 Exosomes/extracellular vesicles. Exosomes or small extracellular vesicles (sEVs) are phospholipid-based NPs secreted by cells. These nanovesicles $(30-150 \mathrm{~nm})$ are formed intracellularly by endosomal multivesicle bodies and are subsequently released from cells by exocytosis. Hence, unlike synthetic vesicles, their surface contains several membrane proteins (Fig. 24A). Additionally, sEVs contain several cytosolic compounds - such as nucleic acids, proteins and lipids - which are transported between cells. ${ }^{338}$ More recently, EVs have been proposed as drug delivery systems, ${ }^{339}$ and hence interest in studying the in vivo distribution of these nanomedicines has subsequently increased. Despite this, there are still relatively few examples of radiolabelled exosomes/sEVs (Table 9).

Similarly to cells, a key consideration for radiolabelling cellderived EVs, is that proteins on their surface can be utilised for functionalisation (Fig. 24A). Hence several groups have reported chelator-based labelling of the surface of exosomes. Shi et al. reported that the bifunctional chelator $p$-SCN-BzNOTA could be conjugated to the surface of EVs via free amines present on the surface membrane. This allowed $>95 \%$ RCY and high serum stability ( $>80 \%$ at $24 \mathrm{~h}) \cdot{ }^{344}$ Similarly, Banerjee et al. conjugated a DO3A-maleimide to the surface of EVs via free thiols present. However, RCYs were relatively low (ca. 16-25\%), and in vivo PET imaging showed consistently high uptake in the bladder which peaked at $25 \%$ ID per $g$ at $3 \mathrm{~h}$; indicating that release of the bioconjugate from the EVs may have occurred (Fig. 24C). ${ }^{341}$ The presence of proteins on the EV surface also allows the direct radioiodination via electrophilic aromatic substitution on tyrosine residues (Fig. 24A). ${ }^{342,347}$ However, in each reported example uptake of radioactivity in 

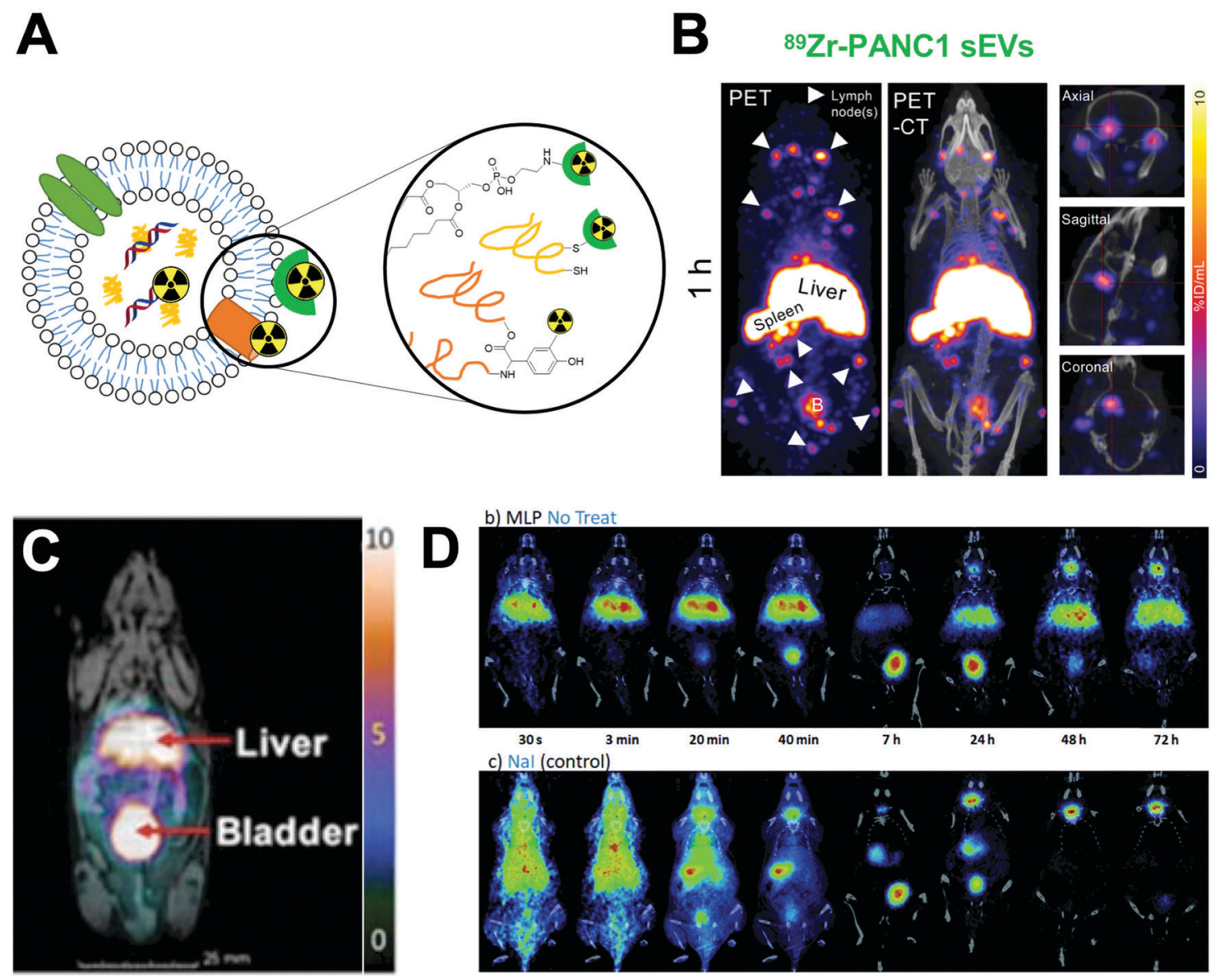

Fig. 24 (A) Schematic representation of the various methods used to radiolabel extracellular vesicles. Chelators can be attached to the surface via conjugation to phospholipids or protein residues, or radionuclides can be incorporated inside via ionophores. (B) Representative MIP PET/CT images of radiolabelled ${ }^{89} \mathrm{Zr}$-labelled PANC1 exosomes in healthy mice; showing signal in liver, spleen, several lymph nodes (arrowheads), and brain; adapted from Khan et al. ${ }^{340}$ (C) PET/MRI of ${ }^{64} \mathrm{Cu}$-labelled sEVs in healthy mice. Adapted from Banerjee et al. ${ }^{341}$ (D) PET-CT coronal images obtained at different time points of ${ }^{124}$ l-labelled EVs in healthy mice (top) and free $\left.{ }^{124}{ }^{12}\right]$ Nal (bottom). Release of iodine can be seen at later timepoint, resulting in thyroid signal. Adapted from Royo et al. ${ }^{342}$

Table 9 Table summarising the methods, radioisotopes and techniques employed to radiolabel extracellular vesicles

\begin{tabular}{|c|c|c|c|}
\hline Radiolabelling method & Radionuclide & Radiolabelling mediator & Ref. \\
\hline \multirow[t]{3}{*}{ Chelator-based } & ${ }^{111}$ In & DTPA & 343 \\
\hline & ${ }^{64} \mathrm{Cu}$ & DOTA & 344 \\
\hline & & DO3A & 341 \\
\hline \multirow[t]{5}{*}{ Non-chelator } & ${ }^{125} \mathrm{I}$ & (3- ${ }^{125}$ I-Iodobenzoyl)norbiotinamide & 345 and 346 \\
\hline & ${ }^{124} \mathrm{I}$ & Iodination tube & 342 \\
\hline & ${ }^{131} \mathrm{I}$ & Iodination tube & 347 \\
\hline & ${ }^{99 \mathrm{~m}_{\mathrm{Tc}}}$ & Via ${ }^{99 \mathrm{~m}} \mathrm{Tc}$ tricarbonyl & 348 \\
\hline & & Direct labelling with $\mathrm{SnCl}_{2}$ reduction & $349-351$ \\
\hline \multirow[t]{3}{*}{ Ionophore-based } & ${ }^{111}$ In & Tropolone & 343 \\
\hline & & Oxine & 352 \\
\hline & ${ }^{89} \mathrm{Zr}$ & Oxine & 340 \\
\hline Remote loading & ${ }^{99 m_{\mathrm{Tc}}}$ & НMPAO & 353 \\
\hline
\end{tabular}

the thyroid was observed, suggesting instability of the radiolabel (Fig. 24D). Hence, direct labelling of EVs with radioiodine may not be as appropriate compared with other methods. As with liposomes, several groups have reported the chelate-free direct labelling of different extracellular vesicles (EVs)/exosomes with reduced ${ }^{99 \mathrm{~m}} \mathrm{Tc} .{ }^{349-351} \mathrm{RCYs}>95 \%$ were consistently reported with high serum stability ( $>90 \%$ ) reported up to $48 \mathrm{~h}$. In vivo SPECT/CT imaging showed that this labelling method was stable 
with minor uptake in the thyroid compared with pertechnetate. ${ }^{349}$ Taking into account the important role that surface proteins have in the biological behaviour of EVs, a potential disadvantage of targeting these proteins for radiolabelling EV surface proteins is the possibility of affecting their structure and function.

Due to their lipid bilayer, EVs are also capable of being radiolabelled using radio-ionophores, with the radiometal binding to proteins in the exosome core (Section 4.3.1 and Fig. 24A). This was first reported by Smyth et al. who used the $\left[{ }^{111}\right.$ In] In-oxine methodology to label PC3 and MCF-7 cell derived exosomes with labelling efficiencies between $67-81 \%{ }^{352}$ In a similar study, Faruqu et al. radiolabelled exosomes using the ${ }^{111}$ In-tropolone radio-ionophore complex, and compared this to labelling using a DTPA chelator conjugated to the surface. ${ }^{343}$ The radio-ionophore method was shown to be inferior to the surface labelling both with regards to radiolabelling and serum stability. ${ }^{111}$ In-tropolone labelled exosomes with just $4 \%$ LE and demonstrated only $14 \%$ serum stability after $24 \mathrm{~h}$. This may be the result of the relatively high stability of ${ }^{111}$ In-tropolone that may have not dissociated inside the EVs. Finally, Hwang et al. reported the labelling of exosomes using remote loading with $\left[{ }^{99 \mathrm{~m}} \mathrm{Tc}\right] \mathrm{Tc}$ HMPAO which was facilitated by the presence of GSH inside the EVs. ${ }^{353}$ The radiolabelled EVs, had high ( $c a .>90 \%$ stability) in serum up to $5 \mathrm{~h}$. However, in vivo SPECT/CT and ex vivo biodistribution of the labelled EVs showed uptake in the salivary glands ( $c a .15 \%$ ID per $\mathrm{g}$ after $3 \mathrm{~h}$ ), suggesting release of ${ }^{99 \mathrm{~m}} \mathrm{Tc}$ from the EVs occurred. ${ }^{353}$

4.5.3 Protein-based nanoparticles. Protein-based nanomedicines offer several beneficial properties including their biodegradability, highly tunable platform and their amphiphilic nature - allowing favourable interactions with drugs. ${ }^{354}$
Furthermore, the use of proteins can instil the nanomedicines with more favourable properties for drug delivery, such as increased target delivery. ${ }^{355}$ A key example of protein-based nanomedicines is NP albumin-bound paclitaxel - known as Abraxane $^{\circledR}$ - which was approved by the FDA for the treatment of several types of solid tumours. The conjugation of paclitaxel to albumin increases the blood half-life of the drug, and overcomes the issues of drug solubility without the need for organic solvent based formulations - which had been associated with several severe and sometimes fatal side effects. ${ }^{9}$ As with other nanomedicines discussed, understanding the biodistribution and pharmacokinetics of these drugs can be highly beneficial for their clinical translation. Hence, several groups have radiolabelled protein-based NPs for in vivo imaging. Table 10 summarises the different radiolabelling methodologies used with protein-based NPs.

A large portion of the radiolabelling of protein-based NPs has been carried out with serum albumin (SA) NPs. Jain et al. reported the direct radiolabelling of SA NPs using ${ }^{99 \mathrm{~m}} \mathrm{Tc}$ via $\mathrm{SnCl}_{2}$ reduction which allowed 98\% LE and 90\% stability in PBS up to 24 h. $^{356}$ A couple of groups reported the ${ }^{99 m} \mathrm{Tc}$ labelling of the SA NPs conjugated with porphyrin photosensitising agents. ${ }^{360,361}$ Both studies demonstrated high labelling efficiencies $>90 \%$, however, no evidence was provided showing ${ }^{99 \mathrm{~m}} \mathrm{Tc}$ was bound to the porphyrin, as opposed to directly to the albumin. The direct radioiodination (Fig. 25A) of SA particles has also been reported by Yi et al. using ${ }^{125} \mathrm{I}$ and ${ }^{131}$ I for SPECT/ CT and therapy respectively. ${ }^{182}$ However, no radiolabelling yields were reported, and SPECT/CT in mice showed large amounts of thyroid uptake at $3 \mathrm{~d}$ p.i. suggesting deiodination from the NPs (Fig. 25B). ${ }^{182}$ A chelator-based method for SA NP

Table 10 Table summarising the methods, radioisotopes and techniques employed to radiolabel protein-based nanoparticles, viral nanoparticles and bacteriophages

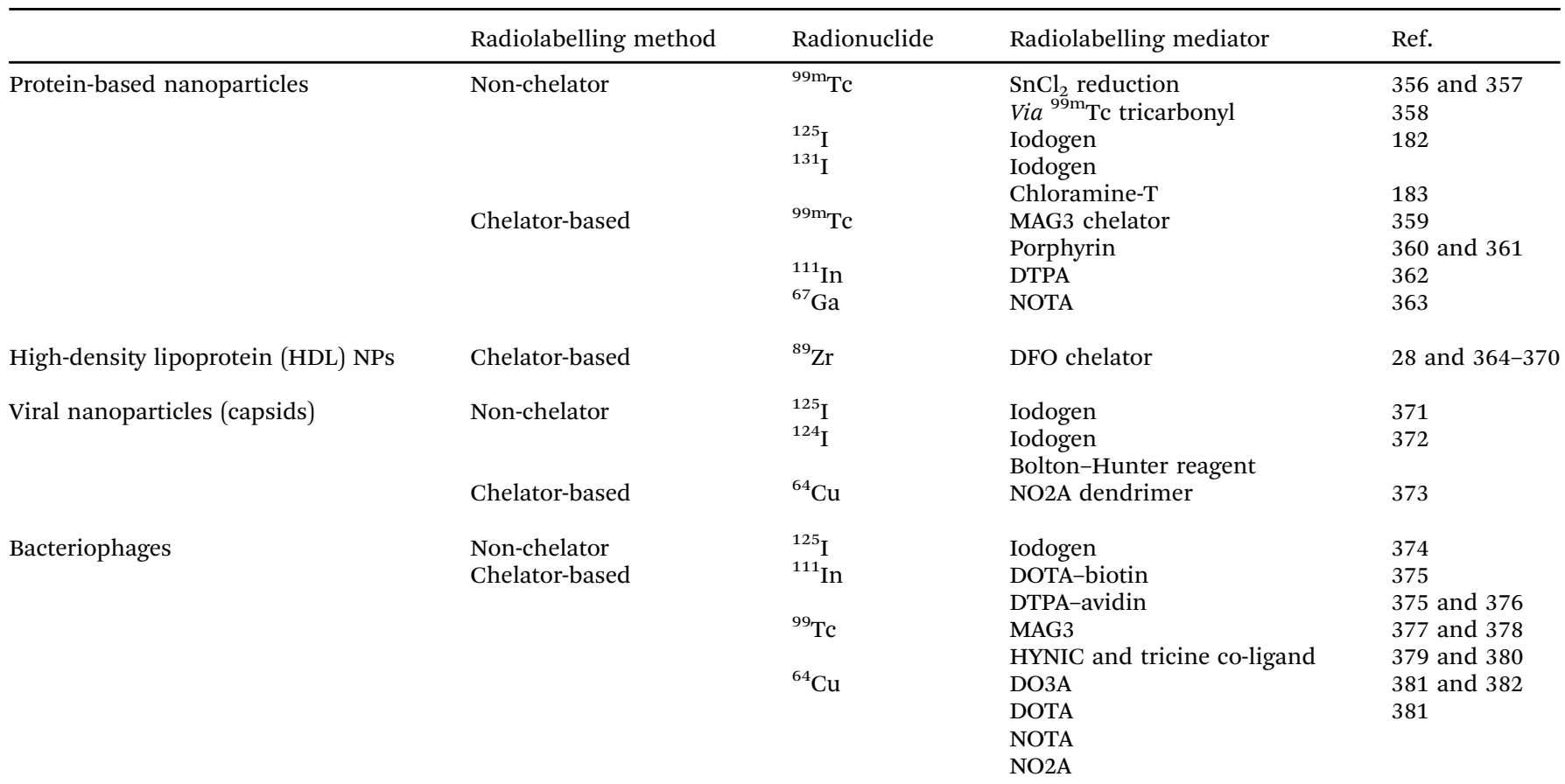



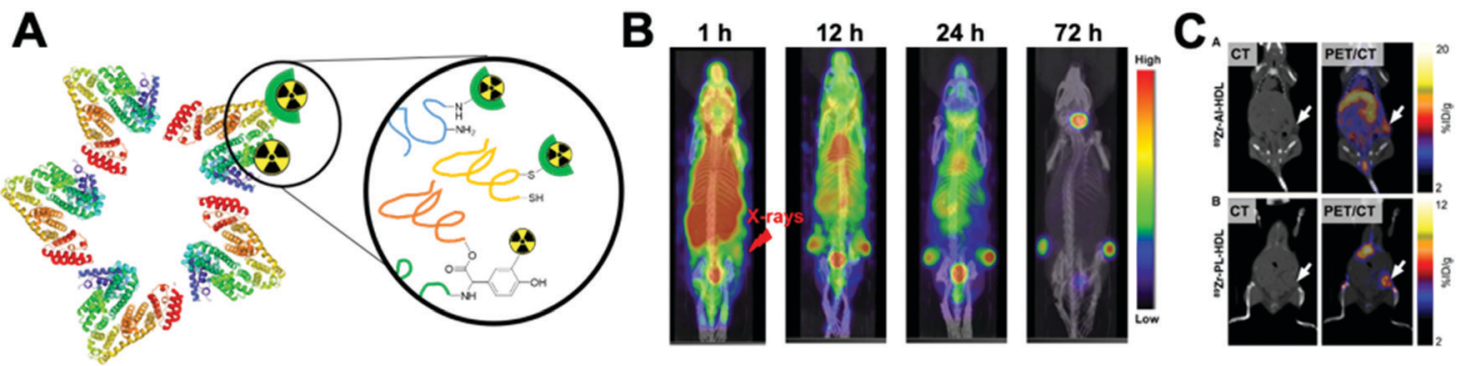

Fig. 25 (A) Schematic representation of the methods used to radiolabel protein-based nanoparticles. Chelators can be conjugated to the nanoparticles via amine or thiol residues on the proteins, or tyrosine residues can be radiohalogenated. (B) SPECT/CT images of ${ }^{125}$ I-HSA in a mouse tumour model. Adapted from Yi et al. ${ }^{182}$ (C) CT (left) and PET/CT fusion (right) images of ${ }^{89} \mathrm{Zr}$-apoAl-DFO-HDL (top) and ${ }^{89} \mathrm{Zr}$-PL-DFO-HDL (bottom) in mice bearing orthotopic 4T1 tumour. Adapted from Pérez-Medina et al. ${ }^{364}$

radiolabelling was reported by Woods et al. who conjugated albumin with $p$-BCS-Bz-DTPA which was then labelled with ${ }^{111}$ In. This was then used to synthesise the albumin NPs with an efficiency of $67 \%$, showing $>97 \%$ stability in serum for $48 \mathrm{~h}^{362}$ Despite this excellent in vitro RCS, the long radiolabelling procedure involving the synthesis of labelled albumin followed by formulation of the particles (overnight) is a potential drawback.

Several other protein-based NPs have been radiolabelled. Yang et al. reported the radiolabelling of self-assembled protein NPs based on polypeptides designed to contain His-tags for labelling with ${ }^{99 \mathrm{~m}} \mathrm{Tc}$ via the tricarbonyl core. ${ }^{358}$ Ferritin-based nanocages were radiolabelled by Liang et al using ${ }^{99 \mathrm{~m}} \mathrm{Tc}$ using a $^{\mathrm{MAG}_{3}}$ conjugated chelator via an NHS ester. ${ }^{359}$ Additionally, Gil et al reported the radiolabelling of casein NPs with ${ }^{67} \mathrm{Ga}$ via the conjugation of $p$-SCN-Bn-NOTA. ${ }^{363}$ A highly robust method for radiolabelling high-density lipoprotein (HDL) based NPs was described by Mulder and collaborators who radiolabelled HDL NPs with ${ }^{89} \mathrm{Zr}$ using the chelator DFO. ${ }^{28,364-370}$ Interestingly, a number of their studies showed that the placement of chelator had significant effects on in vitro stability and in vivo biodistribution of the particles. ${ }^{28,364,365}$ A key study compared the properties of radiolabelled HDL-NPs which were conjugated with $p$-SCN-DFO either to the phospholipid (PL-DFO NPs) layer or the HDL (apoA-I-DFO). ${ }^{364}$ The PL-DFO NPs showed lower RCYs than the apoA-I-DFO NPs (79\% and 94\% respectively). Large differences were observed in vivo (Fig. 25C); with PL-DFO NPs having a nearly 3 -fold lower blood half-life compared with the apoA-I-DFO NPs, and had larger amounts of bone uptake (17\% \& 4\% respectively) - indicating loss of the ${ }^{89} \mathrm{Zr}$. Additionally, apoA-I-DFO NPs showed that $28 \%$ of bone uptake of being associated with bone marrow, whereas this was only $c a .4 \%$ for the phospholipid labelled particles. ${ }^{364}$

4.5.3.1 Viral nanoparticles. Viral nanoparticles (VNPs) refers to several types of nanomaterials; such as plant viruses, bacteriophages and animal viruses. The application of VNPs, as well as virus-like NPs (which do not contain viral genomes), for drug delivery is of growing interest; due to their biocompatibility, ease of functionalisation and increased cellular uptake. ${ }^{383,384}$ Additionally, viral vectors are also being explored for gene delivery and therapy. ${ }^{385}$ VNPs can easily be modified to incorporate radiolabels, allowing their in vivo tracking using radionuclide imaging techniques. The radiolabelling methods used with VNPs are summarised in Table 10.

$\mathrm{Wu}$ et al. described the radioiodination of the viral NP tobacco mosaic virus, with ${ }^{125} \mathrm{I} .{ }^{371}$ This was performed using the iodogen method, with radiolabelling suggested to occur on tyrosine residues present on the VNPs (Fig. 26A). ${ }^{371}$ Similarly, Kothari et al. compared two different methods for the radioiodination of adeno-associated virus (AAV) capsids with ${ }^{124} \mathrm{I}^{372}$ Radiolabelling efficiencies were generally low, but higher with the iodogen method (10-18\%) compared with $1.0-4.5 \%$ when using the Bolton-Hunter reagent to label protein amine residues. Chelator-based methods have also been used to radiolabel VNPs. In particular, Seo et al. developed a method to radiolabel AAV capsids using multimeric NO2A bioconjugate platforms. $^{373}$ The multichelator systems contained eight NO2A rings attached to with either a transcyclooctene (TCO) or maleimide to allow conjugation through either a tetrazinemodified amine group or cysteine residue on the AAV surface (Fig. 26B and C). Both multimers allowed > 99\% RCYs and high molar activity compared to single chelator systems, but labelling efficiencies of the AAVs were low (2-7.5\%) with both bioconjugate systems. $^{373}$

Several reports have also investigated the radiolabelling of bacteriophages (Table 10). ${ }^{381}$ An interesting study by Holman et al. reported the radiolabelling of Pseudonamas bacteriophages with ${ }^{99 \mathrm{~m}}$ Tc using the HYNIC chelator. ${ }^{380}$ HYNIC was conjugated using an NHS ester derivative, but it was found that all but the briefest reaction $(\leq 3 \mathrm{~min}$ ) resulted in loss of infectivity of the phage. However, optimised conditions - which retained infectivity of the phage - radiolabelled the phage with 95\% RCY using the co-ligand tricine. ${ }^{380}$ This highlights the need to ensure that the radiolabelling procedure of viral capsids does not affect their biological function, and how optimisation of the protocol can help mitigate this.

4.5.4 Polymeric micelles. Polymeric micelles are made up of amphiphilic block co-polymers units containing a hydrophilic polymer (e.g. PEG) and hydrophobic drug loading block. The properties of the co-polymers allow them to assemble into NPs; with a hydrophilic shell surrounding the more hydrophobic core, which can encapsulate a variety of drugs during 
A

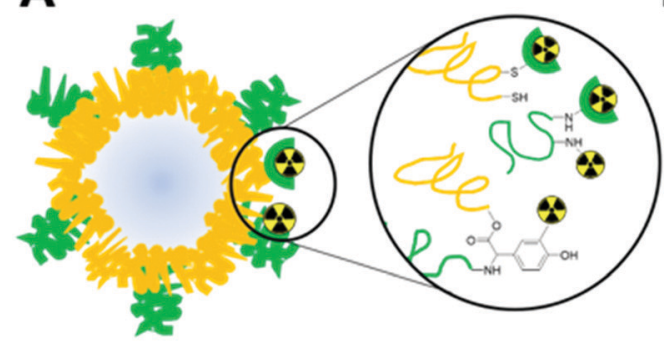

B

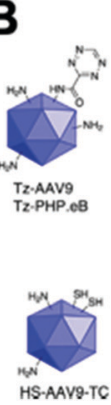

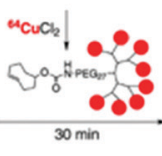

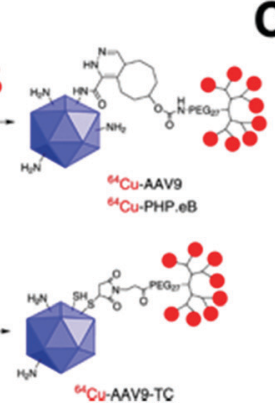

C

"aACu-Aavg "acu-AAV9-TC "BCu-PHP.eB

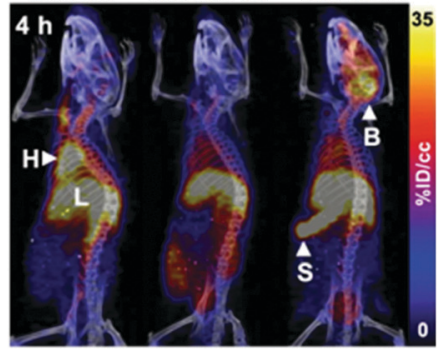

Fig. 26 (A) Schematic representation of the methods used to radiolabel viral nanoparticles. Chelators can be conjugated to the nanoparticles via amine or thiol residues on the proteins, or radiohalogens can be attached via free amine or tyrosine residues. (B) Surface modification with multichelators (MC) on lysine residues in capsids (top) or the site-specific radiolabeling on cysteine residues in capsids via the multichelator-maleimide conjugate (bottom). (C) Representative PET/CT images of ${ }^{64} \mathrm{Cu}$-labelled viral capsids in healthy C57BL/6 mice. Adapted from Seo et al. ${ }^{373}$
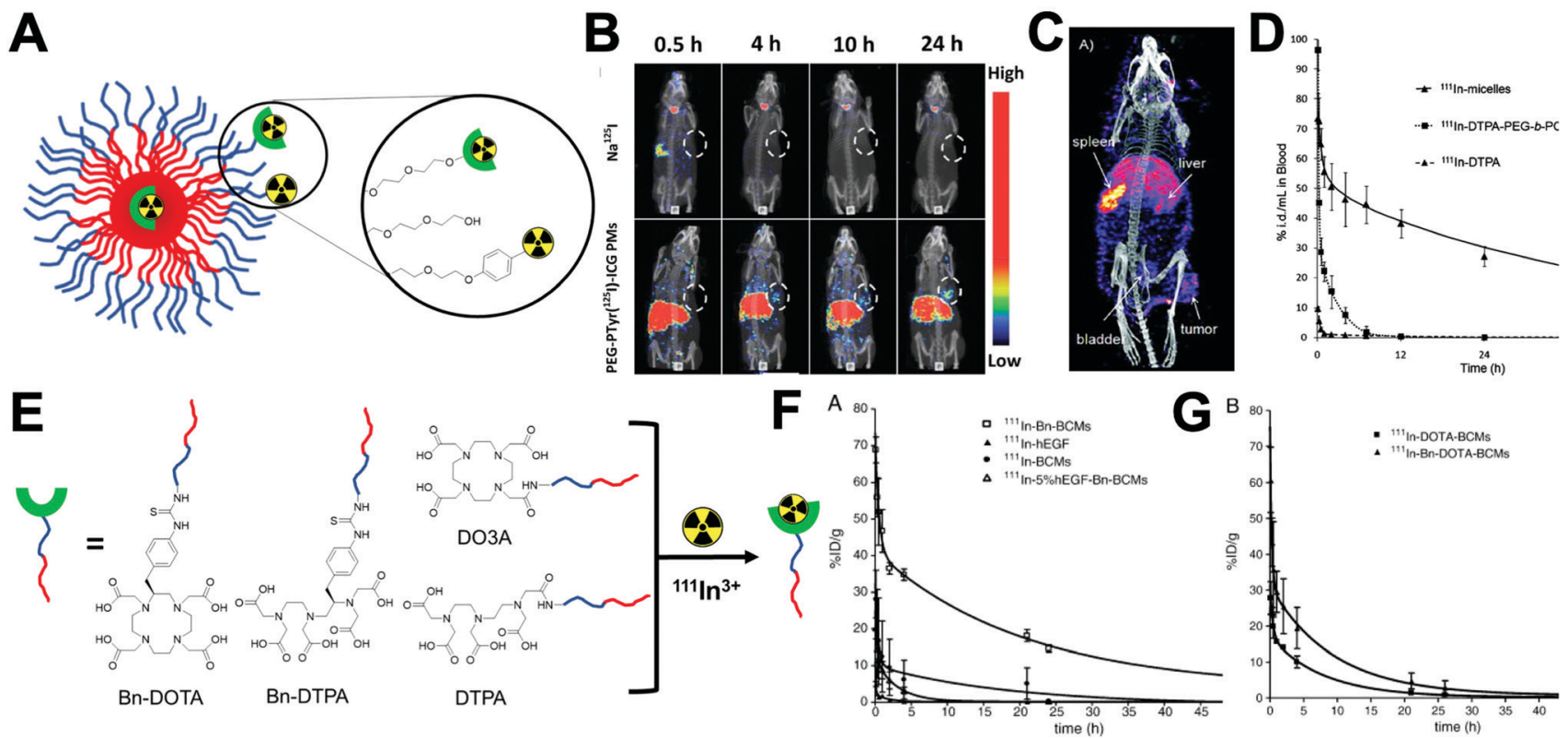

Fig. 27 (A) Schematic representation of the methods of radiolabelling polymeric micelles. Chelators or radiohalogens can be attached to the surface or radionuclides can be trapped inside the micelle core. (B) Representative SPECT-CT images of ${ }^{125}$ I-radiolabelled polymeric micelles (bottom) along with free $\left[{ }^{125}\right] \mathrm{Nal}$ at various timepoints in a tumour mouse model. Adapted from Yang et al. ${ }^{170}$ (C) MIP and sagittal image of tissue accumulation of ${ }^{111}$ In-micelles 48 h p.i. in a breast cancer tumour xenograft. Adapted from Hoang et al. ${ }^{388}$ (D) Comparison of the pharmacokinetics of ${ }^{111}$ In-labelled micelles with the free PEG-b-PCL polymer and the ${ }^{111}$ In-DTPA complex. Adapted from Hoang et al. ${ }^{388}$ (E) Graphical representation of the methodology deployed by Fonge et al. comparing the effect of different BFCs on the pharmacokinetics of radiolabelled micelles. (F) Blood pharmacokinetics of $60 \mathrm{~nm}$ ${ }^{111}$ In-labelled micelles in mice bearing human breast cancer xenografts labelled with ${ }^{111}$ In via $p$-SCN-Bn-DTPA $\left({ }^{111}\right.$ In-Bn-BCMs) or DTPA bis-anhydride $\left({ }^{111} \mathrm{In}-\mathrm{BCMs}\right)$. Blood clearance curve of micelles containing $5 \mathrm{~mol} \% \mathrm{hEGF}$ targeted BCMs is also shown but not discussed. (G) Blood pharmacokinetics in the same model as above with micelles labelled with ${ }^{111}$ In via $p-S C N-B n-D O T A\left({ }^{111}\right.$ In-Bn-DOTA-BCMs) or NHS-DOTA $\left({ }^{111}\right.$ In-DOTA-BCMs). Adapted from Fonge et al. ${ }^{389}$

formulation (Fig. 27A). Block co-polymers are highly tuneable and can be modified with a variety of molecules which will then be present on the hydrophilic shell, allowing control of the distribution and function of the NPs in vivo. Polymeric micelles have been widely explored as nanomedicines, ${ }^{386,387}$ with several formulations in clinical trials. ${ }^{9}$ Several different methods have been applied to the radiolabelling of polymeric micelles, which are summarised in Table 11.

As with other NP types, several studies have reported the radiolabelling of polymeric micelles with ${ }^{99 \mathrm{~m}} \mathrm{Tc}$ using the direct labelling method. ${ }^{390-398}$ Once again, this was shown to be a robust technique; with reported RCYs generally being $>95 \%$ under optimised conditions and serum stability $c a .98 \%$ after $24 \mathrm{~h} .{ }^{395-397}$ The radioiodination of polymeric micelles has also been reported. Kao et al. reported the radiolabelling of co-polymer based micelles containing a benzyl group allowing radiolabelling with ${ }^{131} \mathrm{I}$ using the chloramine-T method (Fig. 27A) ${ }^{173}$ RCYs were $55 \%$, but instability was observed in serum (53\% after $48 \mathrm{~h}$ ). Additionally, in vivo stability could not be assessed as the authors blocked thyroid/stomach uptake by 
Table 11 Table summarising the methods, radioisotopes and techniques employed to radiolabel polymeric micelles

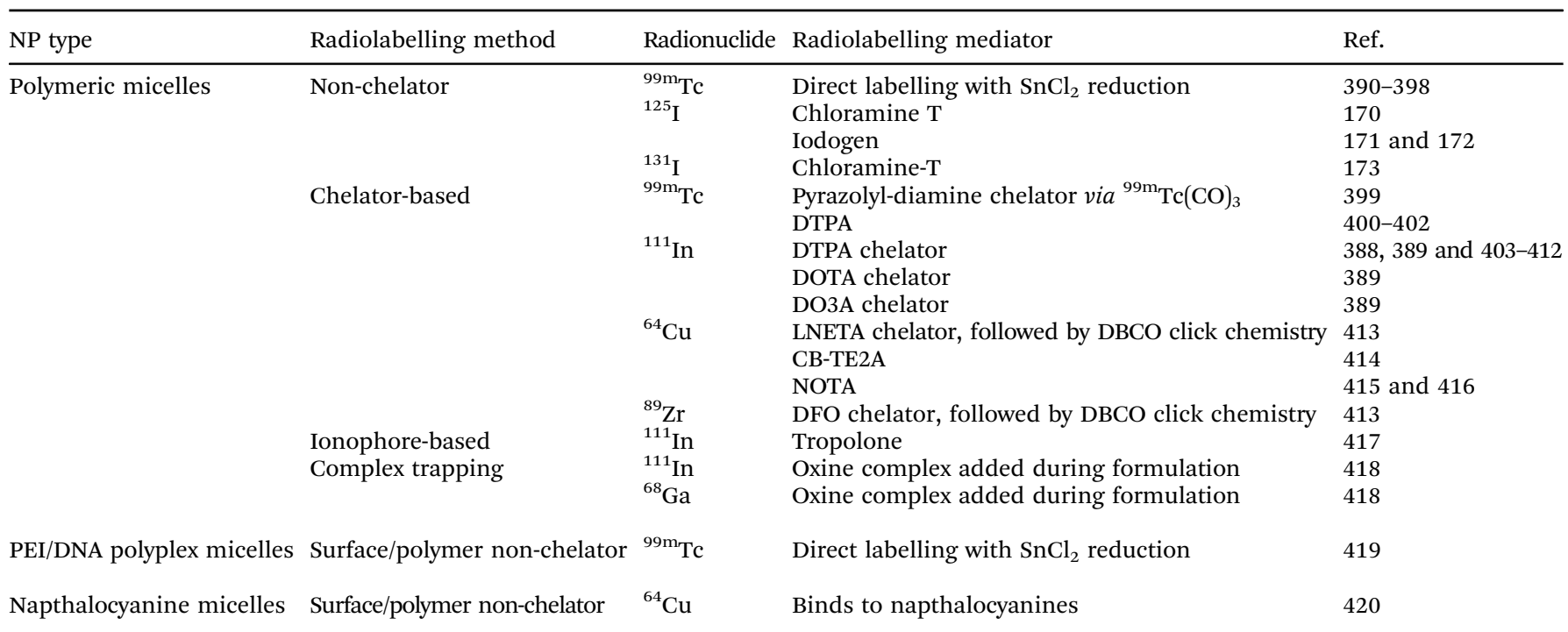

the injection of non-radioactive iodide/perchlorate. ${ }^{173}$ A similar method was described by Yang et al. who synthesised micelles labelled with ${ }^{125} \mathrm{I}$, facilitated by binding to tyrosine residues via chloramine-T. ${ }^{170}$ This allowed RCYs of $77 \%$ and $98 \%$ stability in mouse plasma up to $48 \mathrm{~h}$. Furthermore, in vivo stability also seemed high with little thyroid uptake observed by SPECT/CT imaging (Fig. 27B). ${ }^{170}$ One potential drawback of these reports was the need to radiolabel the polymer first, and then perform the synthesis of the micelles. This process can take long periods of time $\left(>12 \mathrm{~h}\right.$ in the study by Yang $^{170}$ ) which could limit its adoption in clinical setups. Radiolabelling of already formulated micelles with radioiodine has been described. Zhan and collaborators reported using the iodogen method with ${ }^{125}$ I to label PEG-PLA micelles conjugated with targeting peptides. $^{171,172}$

Alternatively, several groups have used chelator-based methods for radiolabelling polymeric micelles (Fig. 27A and Table 11). Hoang et al. highlighted a key consideration for radiolabelling micelles: the biodistribution of the 'free' copolymer. $^{388}$ The authors compared the in vivo distribution of the ${ }^{111}$ In-labelled micelles with the single co-polymer. Whilst the pharmacokinetics were clearly distinct with hugely different blood half-lives $(29 \mathrm{~h}$ and $2 \mathrm{~h}$ for the micelles and polymer, respectively; Fig. 27D), the ex vivo biodistribution showed near identical uptake in the liver ( $c a .12 \%$ ID per $\mathrm{g}$ ) after $48 \mathrm{~h}$ p.i. ${ }^{388}$ Hence, release of the polymer after micelle degradation may contribute to liver uptake observed (Fig. 27C). In another interesting study, Fonge et al. compared the effect of different BFCs on the pharmacokinetics of radiolabelled micelles using ${ }^{111}$ In. ${ }^{389}$ The co-polymers were conjugated with $p$-SCN-Bn-DTPA (DTPA-Bn), DTPA anhydride (DTPA), $p$-SCN-Bn-DTPA (DOTA$\mathrm{Bn}$ ) or with DOTA (DO3A, Fig. 27E), labelled with ${ }^{111}$ In and then used to formulate the polymeric micelles. Several differences were seen in vivo. Firstly, each formulation had different blood half-lives with DTPA-Bn having the highest $(25 \mathrm{~h})$ - the $t_{1 / 2}$ for DTPA, DOTA-Bn and DO3A were $12 \mathrm{~h}, 9 \mathrm{~h}$ and $15 \mathrm{~h}$ respectively
(Fig. $27 \mathrm{~F}$ and $\mathrm{G}$ ). Ex vivo biodistribution at $48 \mathrm{~h}$ in tumourbearing mice also showed distinct differences in uptake. The DTPA-Bn had the highest uptake in all organs of interest with $32 \%$ ID per $g$ in the liver, $15 \%$ ID per $g$ in the spleen and $4 \%$ ID per $g$ in the tumour. Compared with $18 \%$ ID per $g, 1 \%$ ID per $g$ and $<1 \%$ ID per $g$ for the DTPA labelled micelles in the liver, spleen and tumour respectively. Despite their differences in pharmacokinetics, the two DOTA based formulations had similar uptake at $48 \mathrm{~h}$ with $c a$. 10\% ID per g, 2\% ID per $\mathrm{g}$ and $2 \%$ ID per $g$ in the liver, spleen and tumour respectively. ${ }^{389}$ These results highlight that chelator-based radiolabelling approaches can potentially have large effects on the in vivo behaviour of the radiolabelled particles.

Laan et al. reported the radiolabelling of micelles using the radio-ionophore $\left.\left[{ }^{111} \mathrm{In}\right][\operatorname{In} \text { (tropolone) })_{3}\right]$ via either the trapping of the lipophilic complex during the formation of the NPs, or by labelling preformed micelles. ${ }^{417}$ The hypothesis was that the lipophilic complex would become trapped within the micellar core. Both methods showed relatively low labelling efficiency with $32 \%$ LE during micelle formation and $22 \%$ LE of preformed micelles. Incubation in serum showed $c a$. 20\% loss of activity after $2 \mathrm{~d}^{417}$ Similarly, de la Fuente et al. reported the radiolabelling of micelles via the addition of either $\left[{ }^{68} \mathrm{Ga}\right] \mathrm{Ga}$ oxine and $\left[{ }^{111} \mathrm{In}\right]$ In-oxine complexes during formulation. ${ }^{418}$ However, release of the oxine complexes was shown to occur rapidly in vivo; such that the ex vivo biodistribution of the labelled micelles and the administered oxine complexes as a control were nearly identical.

4.5.5 Dendrimers. Dendrimers are nano-sized macromolecules consisting of a core (single atom or molecule) to which repeating units known as branches are attached (Fig. 28A). The branches will have at least one branch junction, which with repetition results in a series of layers - known as "generations" - usually denoted by a number (i.e. G1, G2, G3,...). Due to this unique structure, dendrimers have well-defined sizes and are highly uniform. Furthermore, their structure is highly tuneable 
and often biocompatible, making them attractive platforms for drug delivery. ${ }^{421}$ Additionally, their architecture allows - or can easily be modified to allow - radiolabels for the assessment of the in vivo behaviour (see Table 12).

The overwhelming majority of examples of dendrimer radiolabelling has been performed with poly(amidoamine) (PAMAM) based dendrimers. A common technique employed with this type of dendrimer is the conjugation of a chelator to amine residues present on the polymer (Fig. 28A). For example, several reports used DTPA-based conjugates to radiolabel dendrimers with ${ }^{111} \mathrm{In}^{432-435}$ Sano et al. reported $>99 \%$ RCYs using $p$-SCNBn-DTPA conjugated to PAMAM-based dendrimers. ${ }^{435}$ Furthermore, PAMAM dendrimers have been radiolabelled with the radiotherapeutic isotope ${ }^{177} \mathrm{Lu} .{ }^{427-431}$ using the amine reactive BFCs DOTA-NHS, ${ }^{428}$ and $p$-SCN-Bn-DOTA. ${ }^{429,430}$

The radiolabelling of PAMAM dendrimers with ${ }^{64} \mathrm{Cu}$ has also been performed using the DOTA-NHS BFC system. ${ }^{42,426}$ This allowed RCYs of $c a .85 \%$ and $>93 \%$ serum stability up to $20 \mathrm{~h}^{426}$ Interestingly, Lesniak et al. compared the labelling and biodistribution of ${ }^{64} \mathrm{Cu}$-labelled and ${ }^{111} \mathrm{In}$-labelled dendrimers with this chelate system. ${ }^{422}$ Whilst RCYs with both radiometals were similar $(80 \%)$, discrepancies in the biodistributions of the labelled dendrimers were found between the two. Liver uptake was 6-8 fold higher with ${ }^{64} \mathrm{Cu}$ over $48 \mathrm{~h}$ compared with the ${ }^{111}$ In-labelled NPs, and spleen uptake at $1 \mathrm{~h}$ was $107 \%$ ID per $\mathrm{g}$ - decreasing to $68 \%$ ID per $\mathrm{g}$ at $48 \mathrm{~h}$ - for ${ }^{64} \mathrm{Cu}$-labelled dendrimers - whereas spleen uptake increased overtime for the ${ }^{111}$ In-labelled NPs $(6.4 \%$ ID per $\mathrm{g}$ at $1 \mathrm{~h}$ to $34 \%$ ID per $\mathrm{g}$ at $48 \mathrm{~h}$ p.i.). Furthermore, the ${ }^{111}$ In-labelled dendrimer biodistribution matched more closely with that seen with dendrimers labelled with an optical probe. ${ }^{422}$ These results taken together suggest loss of ${ }^{64} \mathrm{Cu}$ from the dendrimer. Indeed, it is well established the superiority of DOTA as a chelator for ${ }^{111} \mathrm{In}$, compared with ${ }^{64} \mathrm{Cu} .{ }^{94}$ Alternatively, NOTA has often been shown to be a more suitable chelator for ${ }^{64} \mathrm{Cu},{ }^{94,470}$ This study highlights the stark differences in biodistribution that can occur from using different radiometals in the same system, and that chelators should be chosen appropriately to match with each radiometal.

A key study by Valliant and collaborators reported the radiolabelling of various dendrimers with ${ }^{99 \mathrm{~m}} \mathrm{Tc}$ using DPA to

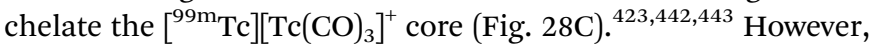
in this case the chelator was conjugated via an alkyl amine to the dendrimer core which had been functionalised with an NHS ester. RCYs of $c a$. $90 \%$ in just 5 min using a microwave synthesis unit were reported - albeit at high temperatures of 80-130 ${ }^{\circ} \mathrm{C}$. Interestingly, the authors noted a reduction in RCY when radiolabelling larger dendrimers with G6 and G7 dendrimers having RCYs of $70 \%$ and $53 \%$, respectively compared with $c a .90 \%$ observed with G5. ${ }^{423}$ This highlights a potential drawback with radiolabelling dendrimers via their core, as opposed to functional groups on the outer layers, wherein increasing dendrimer size potentially renders the chelator less accessible for radiolabelling (Fig. 28C).

The amine residues on PAMAM dendrimers also allow the radioiodination using Bolton-Hunter reagent, ${ }^{152-156}$ as well as another amine-reactive reagent, $N$-succinimidyl 3-iodobenzoate (Fig. 28A). ${ }^{464,465}$ The chloramine-T method was used by several groups to radiolabel dendrimers with ${ }^{125} \mathrm{I},{ }^{157-160}$ and with the PET isotope ${ }^{76} \mathrm{Br},{ }^{184}$ however the dendrimers usually had to be modified with either tyrosine or tyramine residues to allow radiolabelling.
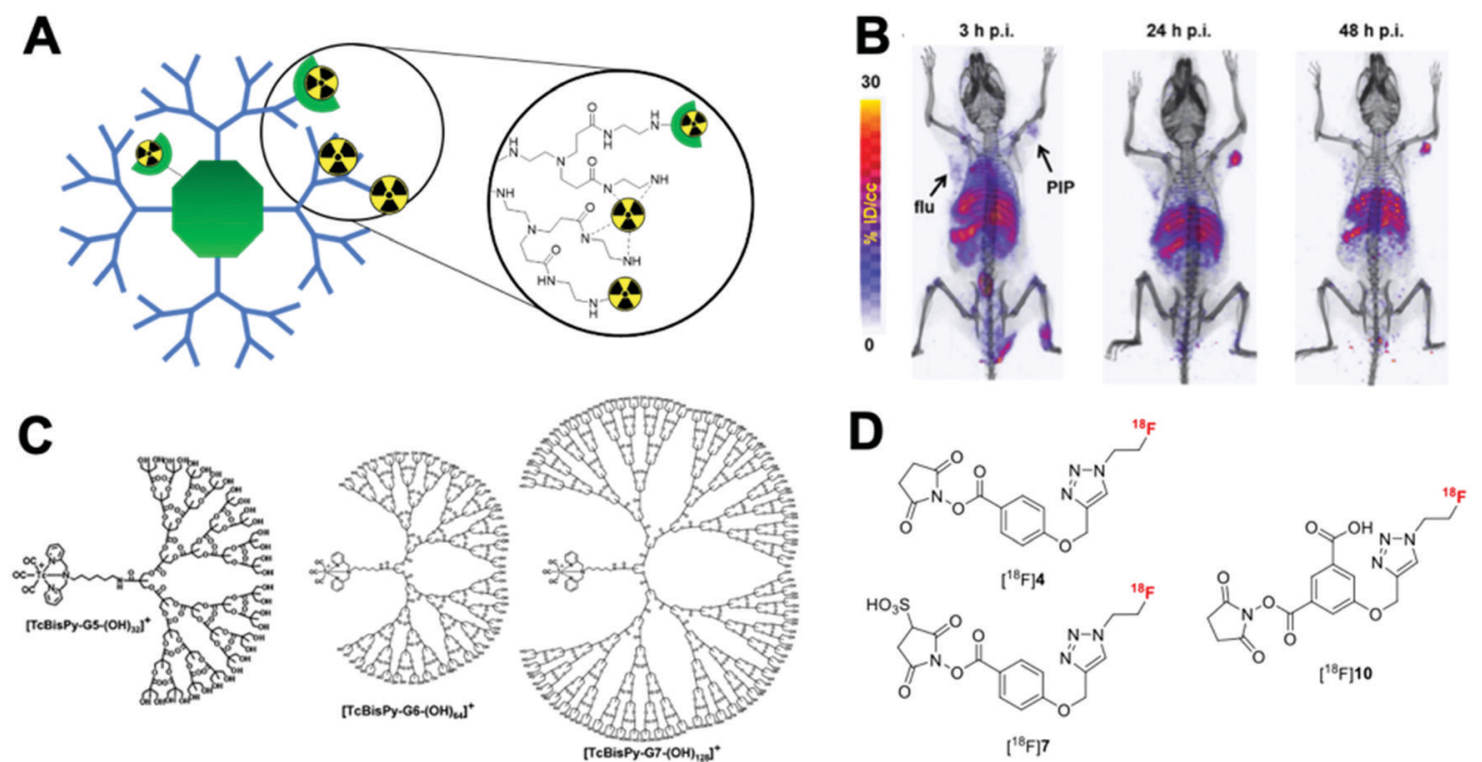

Fig. 28 (A) Schematic representation of methods used to radiolabel dendrimers. Chelators can be attached to the dendrimer core or to the outer layer of PAMAM dendrimers via the free amines. Alternatively, radiometals can be bound directly to free amines present. Finally, radiohalogens can also be attached to the outer layer. (B) Representative PET/CT of radiolabelled PSMA-targeted dendrimers in male NOD-SCID mice bearing PSMA+ PC3 PIP and PSMA - PC3 flu tumour xenografts with ${ }^{64} \mathrm{Cu}$, adapted from Lesniak et al. ${ }^{422}$ (C) Scheme showing the different size dendrimers radiolabelled by Valliant and collaborators. ${ }^{423}$ (D) Chemical structures of the ${ }^{18} \mathrm{~F}$-labelled NHS agents reported by Zhou et al. to radiolabel amine-functionalised PAMAM dendrimers. ${ }^{424}$ 
Table 12 Table summarising the methods, radioisotopes and techniques employed to radiolabel dendrimers

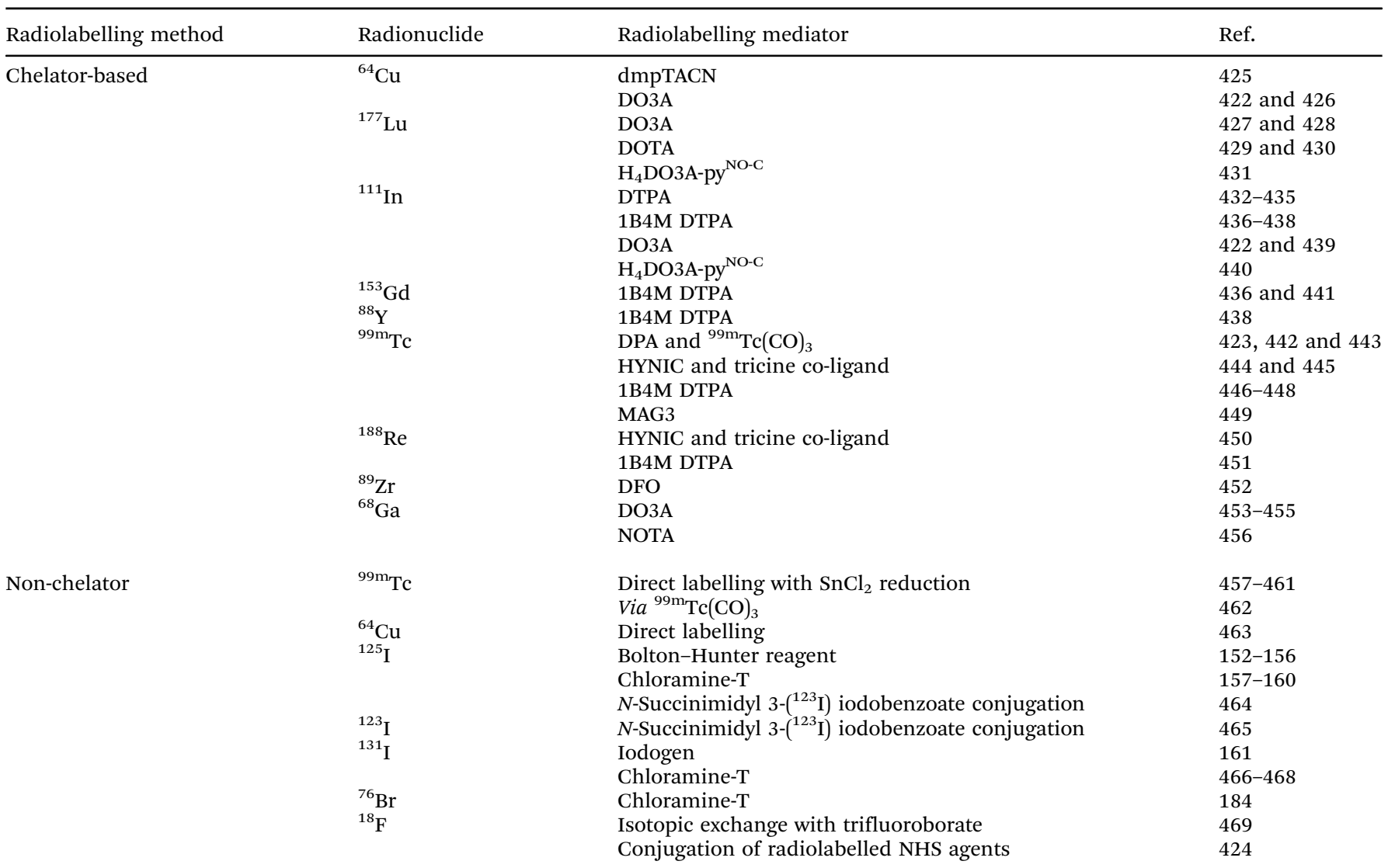

Alternatively, Zhou et al. reported several ${ }^{18} \mathrm{~F}$-labelled NHS agents (Fig. 28D) to radiolabel amine-functionalised PAMAM dendrimers. ${ }^{424}$ RCYs under optimised conditions were $28 \%$, 95\% and $95 \%$ for $\left[{ }^{18} \mathrm{~F}\right] 4,\left[{ }^{18} \mathrm{~F}\right] 7$ and $\left[{ }^{18} \mathrm{~F}\right] 10$ respectively after $5 \mathrm{~min}$, with the RCY with $\left[{ }^{18} \mathrm{~F}\right] 4$ increasing to $71 \%$ after $15 \mathrm{~min}$. The higher labelling efficiency and faster reaction kinetics of $\left[{ }^{18} \mathrm{~F}\right] 7$ and $\left[{ }^{18} \mathrm{~F}\right] 10$ was hypothesised to occur due to electrostatic interactions of the NHS esters with the PAMAM dendrimers. $\left[{ }^{18} \mathrm{~F}\right] 7$ and $\left[{ }^{18} \mathrm{~F}\right] 10$ would become pre-localised to the dendrimers due to the interaction of the sulfonate and carboxylate groups respectively, with the positively charged amines on the dendrimers. ${ }^{424}$

Finally, the direct labelling of PAMAM dendrimers with radiometals has also been reported by several groups. In each case binding of the radiometals was proposed to occur via the amine groups on the dendrimer (Fig. 28A). Once again, the binding of $\left[{ }^{99 m} \mathrm{Tc}\right] \mathrm{TcO}_{4}$ after $\mathrm{SnCl}_{2}$ reduction has been described by several groups, ${ }^{457-461}$ allowing high RCYs and serum stability. Interestingly, Tassano et al. found the

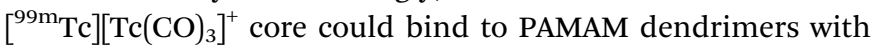
ca. $90 \%$ RCY. ${ }^{462}$ However, the complex was shown to be unstable in a competition assay with histidine; with over $50 \%$ of the activity being transchelated after just $1 \mathrm{~h}^{462}$ The direct labelling of PAMAM dendrimers was also reported with ${ }^{64} \mathrm{Cu}$ by $\mathrm{Xu}$ et $a .^{463}$ Optimised conditions showed that ca. 95\% RCYs could be achieved after just $15 \mathrm{~min}$ at room temp. and $\mathrm{pH} 7$. Labelling at acidic conditions was reduced (30\% at $\mathrm{pH} 3$ ) suggesting that chelation was occurring via the amines on the PAMAM dendrimer, as these amine groups would become more protonated at lower $\mathrm{pH}$. This interaction was shown to be relatively stable with $\mathrm{ca}$. $80 \%$ stability in mouse plasma was after 24 h. $^{463}$

4.5.6 Polymer nanomaterials. A diverse number of other polymer-based nanomaterials have also been radiolabelled using a variety of different methods (Fig. 29 and Table 13). Due to the large number of different types of nanomaterials all with different properties - it is difficult to draw conclusions that apply to most NP platforms. Instead, in this section, general trends in the field will be discussed, along with particular studies that we believe are of special interest.

As with the other NP types discussed previously, several different polymer-based nanomaterials have been radiolabelled using the non-chelator direct labelling method with ${ }^{99 m_{T}}$ Tc. PLGA-based NPs (PLGA-NP), ${ }^{474-486}$ latex NPs, ${ }^{535}$ PLA NPs $^{531,532}$ polydopamine (PDA) NPs, ${ }^{181}$ and poly(anhydride) $\mathrm{NPs}^{536}$ have all been radiolabelled in this way with high (>90\%) RCYs generally reported. The radiolabelling of chitosan-based NPs with this method has also been reported by several groups, resulting in $85-98 \%$ RCYs. ${ }^{497-502}$ Binding of ${ }^{99 \mathrm{~m}} \mathrm{Tc}$ is likely to occur via the free hydroxyl and amines present on the chitosan polymer, however this has not been characterised. The direct labelling of chitosan NPs with ${ }^{64} \mathrm{Cu}$ and ${ }^{89} \mathrm{Zr}$ has also been reported by Fairclough et al. ${ }^{503,504}$ RCYs were $c a$. $72 \%$ for both 

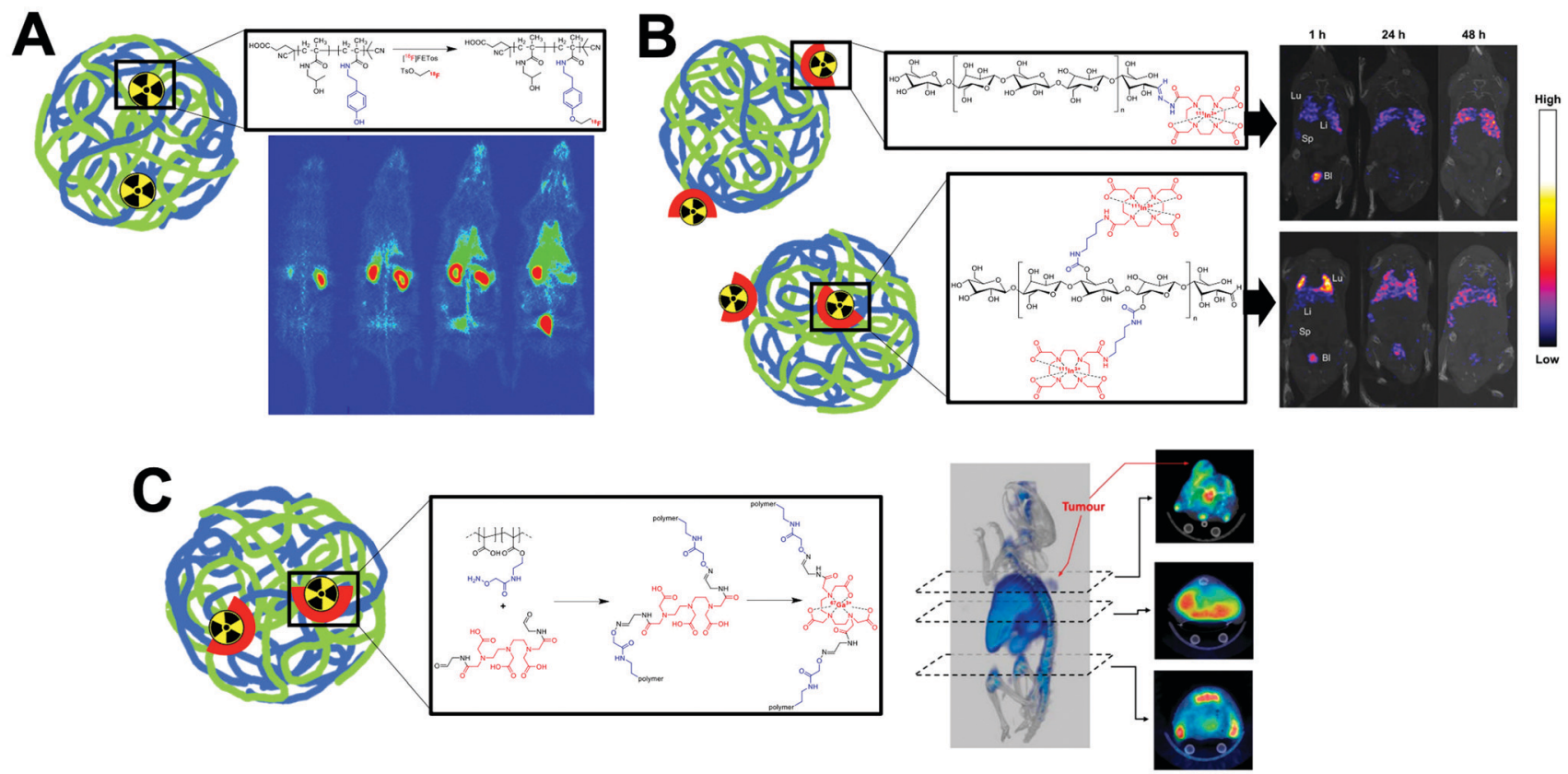

Fig. 29 Figure showing the various ways of incorporating radionuclides into different polymer-based nanomaterials. (A) Radiohalogens can be attached to the polymer backbone. Schematic showing the radiolabelling of $\mathrm{N}$-(2-hydroxypropyl)-methacrylamide (HPMA) with 2 - $\left[{ }^{18} \mathrm{~F}\right] f l u o r o e t h y l-1$-tosylate $\left(\left[^{18} \mathrm{~F}\right] \mathrm{FET}\right.$ (O) $)$ and PET images in healthy rats of the radiolabelled polymer reported by Allmeroth and collaborators. Adapted from Herth et al. ${ }^{471}$ (B) Chelators can be attached to the polymer backbone. Schematic showing the two different bioconjugate strategies used for the radiolabelling of cellulose-based nanoparticles with ${ }^{111}$ In reported by Imlimthan et al - and SPECT/CT in tumour-bearing mice. Adapted from ref. 33. (C) Chelators can be incorporated directly into the polymer structure. Schematic showing the functionalisation of single-chain poly-(methacrylic acid) with a DTPA derivative compound for radiolabelling with ${ }^{67} \mathrm{Ga}$ along with SPECT/CT images in tumour bearing mice. Adapted from Benito et al. ${ }^{472}$

radiometals; with larger MW polymers (>190 $\mathrm{kDa})$ resulting in higher LE for ${ }^{89} \mathrm{Zr}$ over ${ }^{64} \mathrm{Cu}\left(90 \%\right.$ and $72 \%$, respectively). ${ }^{503}$ Fan et al. showed that melanin NPs could be directly labelled with ${ }^{64} \mathrm{Cu}$, which was hypothesised to occur via free hydroxyl and carbonyl groups present on melanin. ${ }^{543,544}$ RCYs of $80 \%$ were achieved under mild condition (after $1 \mathrm{~h}$ at $40{ }^{\circ} \mathrm{C}$ ) with ca. $90 \%$ stability in FBS after $24 \mathrm{~h}$.

Several groups have used radiohalogenation-based reactions for the labelling of polymeric nanomaterials. However, these often involve the modification of the polymer structure first. For examples, Allmeroth and collaborators reported the labelling of $\quad N$-(2-hydroxypropyl)-methacrylamide (HPMA) polymer structures; which were first modified with tyramine, which allowed subsequent reaction with $2-\left[{ }^{18} \mathrm{~F}\right]$ fluoroethyl-1-tosylate $\left(\left[{ }^{18} \mathrm{~F}\right]\right.$ FETos) (Fig. 29A). ${ }^{168,471,493-495}$ This allowed ca. 90\% RCY under optimised conditions. ${ }^{471}$ Wagener et al. compared this method with the radioiodination with ${ }^{131} \mathrm{I}$ using chloramine-T, which allowed $c a .50 \%$ RCY after 4 min. ${ }^{168}$ Interestingly, biodistribution studies of the two radiohalogenated derivatives showed stark differences in organ uptake after $2 \mathrm{~h}$ p.i. Liver uptake of the ${ }^{131} \mathrm{I}$-labelled polymer was 5 -fold that of the ${ }^{18} \mathrm{~F}$ derivative, and was 12 -fold higher in the spleen. Conversely, higher kidney uptake was observed for the ${ }^{18} \mathrm{~F}$-labelled polymer than the ${ }^{131} \mathrm{I}$ derivative ( $c a .5 .5 \%$ and $0.5 \%$ ID per g respectively). Additionally, thyroid uptake ( $c a .22 \%$ ID per g) was observed for the ${ }^{131} \mathrm{I}$ at $24 \mathrm{~h}$ p.i. strongly suggesting significant deiodination in vivo. ${ }^{168}$

The structures of certain polymers can however enable radioiodination reactions without the need for further modification.
Rahmani et al. reported the radioiodination of PLGA-polymethylmethacrylate (PMMA) co-polymer NPs with ${ }^{125}$ I using iodobeads. ${ }^{178}$ Radiolabelling was facilitated by the phenol-containing PMMA, which allowed 95\% RCP after synthesis and purification. The radiolabel was shown to be stable in vivo with $<2 \%$ ID per $\mathrm{g}$ in the thyroid over $24 \mathrm{~h}$ p.i. ${ }^{178}$ Similarly, the phenol residues present on polyvinyl phenol (PVP)-based NPs allow the radioiodination. ${ }^{179,180}$ Simone et al. used iodination beads to radiolabel PVP NPs with both ${ }^{125}$ I and ${ }^{124} \mathrm{I}$, allowing $c a .90 \%$ RCY and $>80 \%$ serum stability over $3 \mathrm{~d}$. However, release of free iodide was observed in vivo with $c a$. 3\% ID observed in the thyroid after 24 h. ${ }^{180}$ Similarly, Tang et al. radiolabelled PEGylated polyvinyl phenol NPs with ${ }^{125}$ I using the chloramine-T method which resulted in $>90 \%$ RCYs. ${ }^{179}$ Stability of the radiolabel was high with $>95 \%$ stability in human serum over $48 \mathrm{~h}$, and $<0.2 \%$ ID uptake in the thyroid of mice observed up to 4 d. ${ }^{179}$ Zhong et al. took advantage of the benzene rings present on polydopamine (PDA) NPs to radiolabel the particles with ${ }^{131} \mathrm{I}$ using chloramine-T - resulting in $70 \% \mathrm{RCY}{ }^{181}$

As well as their reaction with radiohalogens, polymer structures on NPs can also easily facilitate the bioconjugation of chelators for labelling with radiometals. For example, Gracia et al radiolabelled single-chain dextran based NPs with ${ }^{67} \mathrm{Ga}$ by coupling the $\mathrm{BFC} \mathrm{NH}_{2}$-NODAGA to carboxylate residues on the NPs which allowed RCYs of $c a .50 \%$ after. ${ }^{509}$ An interesting study by Imlimthan et al. radiolabelled cellulose-based NPs with ${ }^{111}$ In using two different bioconjugates. ${ }^{33}$ The cellulose polymers were functionalised with a DO3A chelator either via a 
Table 13 Table summarising the methods, radioisotopes and techniques employed to radiolabel polymer-based nanomaterials

\begin{tabular}{|c|c|c|c|c|}
\hline NP type & $\begin{array}{l}\text { Radiolabelling } \\
\text { method }\end{array}$ & Radionuclide & Radiolabelling mediator & Ref. \\
\hline \multirow[t]{8}{*}{ PLGA NPs } & Complex trapping & ${ }^{111}$ In & $\begin{array}{l}\text { Oxine complex added during } \\
\text { formulation }\end{array}$ & 473 \\
\hline & \multirow[t]{3}{*}{ Non-chelator } & ${ }^{99 m_{T}} \mathrm{Tc}$ & Direct labelling with $\mathrm{SnCl}_{2}$ reduction & $474-486$ \\
\hline & & ${ }^{125} \mathrm{I}$ & Iodobeads & 178 \\
\hline & & ${ }^{18} \mathrm{~F}$ & Biotin conjugate after formulation & 487 \\
\hline & \multirow[t]{3}{*}{ Chelator-based } & ${ }^{99 \mathrm{~m}} \mathrm{Tc}$ & DTPA & 488 \\
\hline & & ${ }^{111} \mathrm{In}$ & DTPA chelator & 489 \\
\hline & & ${ }^{177} \mathrm{Lu}$ & DO3A chelator & 490 and 491 \\
\hline & Remote loading & ${ }^{177} \mathrm{Lu}$ & DOTATATE complex & 492 \\
\hline \multirow[t]{4}{*}{ HPMA copolymer NP } & \multirow{4}{*}{ Non-chelator } & ${ }^{18} \mathrm{~F}$ & Tosylate & 168,471 and $493-495$ \\
\hline & & ${ }^{131} \mathrm{I}$ & Chloramine-T & 168 \\
\hline & & ${ }^{125} \mathrm{I}$ & Chloramine-T & 167 \\
\hline & & ${ }^{72 / 74}$ As & Thiol binding & 496 \\
\hline \multirow[t]{4}{*}{ Chitosan NPs } & \multirow[t]{3}{*}{ Non-chelator } & ${ }^{99 \mathrm{~m}_{\mathrm{Tc}}}$ & Direct labelling with $\mathrm{SnCl}_{2}$ reduction & $497-502$ \\
\hline & & ${ }^{64} \mathrm{Cu}$ & Direct labelling & 503 \\
\hline & & ${ }^{89} \mathrm{Zr}$ & Direct labelling & 503 and 504 \\
\hline & Chelator-based & ${ }^{64} \mathrm{Cu}$ & DO3A via DBCO click chem & 505 and 506 \\
\hline Chitosan/polyglutamic acid NPs & Chelator-based & ${ }^{68} \mathrm{Ga}$ & NOTA & 507 \\
\hline $\begin{array}{l}\text { Quaternary ammonium palmitoyl glycol } \\
\text { chitosan (GCPQ) NPs }\end{array}$ & Non-chelator & ${ }^{125} \mathrm{I}$ & Bolton-Hunter reagent & 166 \\
\hline Polyglucose NPs & Non-chelator & ${ }^{18} \mathrm{~F}$ & CuAAC click reaction & 508 \\
\hline Dextran-based single chain polymer NPs & Chelator-based & ${ }^{67} \mathrm{Ga}$ & NODAGA & 509 \\
\hline Dextran NP & Chelator-based & ${ }^{89} \mathrm{Zr}$ & DFO & 510 \\
\hline Cellulose NPs & Chelator-based & ${ }^{111}$ In & DO3A & 33 \\
\hline \multirow[t]{3}{*}{ Shell cross-linked Knedel-like NPs } & \multirow[t]{3}{*}{ Chelator-based } & \multirow[t]{3}{*}{${ }^{64} \mathrm{Cu}$} & TETA chelator & 511 \\
\hline & & & DOTA chelator & $512-515$ \\
\hline & & & DO3A & 512 \\
\hline Hyaluronan NPs & Chelator-based & ${ }^{89} \mathrm{Zr}$ & DFO chelator & 516 \\
\hline PEG chain & Chelator-based & ${ }^{89} \mathrm{Zr}$ & DFO chelator & 517 \\
\hline \multirow[t]{2}{*}{ Nanogel } & Non-chelator & ${ }^{99 \mathrm{~m}_{\mathrm{Tc}}}$ & $\mathrm{SnCl}_{2}$ reduction & 518 \\
\hline & Chelator-based & ${ }^{68} \mathrm{Ga}$ & NOTA & 519 and 520 \\
\hline \multirow[t]{3}{*}{ Polyoxazoline polymer } & Non-chelator & ${ }^{18} \mathrm{~F}$ & Isotopic exchange using SiFA & 521 \\
\hline & Chelator-based & ${ }^{111} \mathrm{In}$ & DOTA chelator & 522 and 523 \\
\hline & & ${ }^{90} \mathrm{Y}$ & DOTA chelator & 523 \\
\hline \multirow[t]{2}{*}{ Caprolactone polymers } & Non-chelator & ${ }^{125} \mathrm{I}$ & Chloramine-T & 162 \\
\hline & Non-chelator & ${ }^{99 \mathrm{~m}_{\mathrm{Tc}}}$ & Direct labelling with $\mathrm{SnCl}_{2}$ reduction & 524 \\
\hline Poly( $\beta$-amino ester) NP & Ionophore-based & ${ }^{111} \mathrm{In}$ & Oxine complex added during formulation & 525 \\
\hline PEG-MA/MMA comb NP & Chelator-based & ${ }^{64} \mathrm{Cu}$ & DO3A chelator & 526 and 527 \\
\hline Poly(maleic anhydride- & Non-chelator & ${ }^{125} \mathrm{I}$ & Iodogen & 174 \\
\hline alt-1-octadecene) NP & & ${ }^{131} \mathrm{I}$ & Iodogen & 174 \\
\hline \multirow[t]{2}{*}{ Poly $(\gamma$-glutamic acid $)$ NPs } & Non-chelator & ${ }^{125} \mathrm{I}$ & Iodogen & 175 \\
\hline & Chelator-based & ${ }^{111} \mathrm{In}$ & DTPA & 528 \\
\hline \multirow[t]{2}{*}{ Polyester-based NPs } & Non-chelator & ${ }^{18} \mathrm{~F}$ & 4- $\left.{ }^{18} \mathrm{~F}\right]$ fluorobenzyl-2-bromoacetamide & 529 \\
\hline & & ${ }^{125} \mathrm{I}$ & Iodobeads & 177 \\
\hline Polythiophene NPs & Chelator-based & ${ }^{99 \mathrm{~m}} \mathrm{Tc}$ & HYNIC with co-ligands (TPPTS and tricine) & 530 \\
\hline PLA NPS & Non-chelator & ${ }^{99 \mathrm{~m}_{\mathrm{Tc}}}$ & Direct labelling with $\mathrm{SnCl}_{2}$ reduction & 531 and 532 \\
\hline & Encapsulated complex & ${ }^{99 \mathrm{~m}_{\mathrm{Tc}}}$ & $\begin{array}{l}\text { HMPAO complex added during } \\
\text { formulation }\end{array}$ & 533 and 534 \\
\hline Latex NPs & Non-chelator & ${ }^{99 \mathrm{~m}_{\mathrm{Tc}}}$ & Direct labelling with $\mathrm{SnCl}_{2}$ reduction & 535 \\
\hline Polyanhydride NPs & Non-chelator & ${ }^{99 \mathrm{~m}_{\mathrm{Tc}}}$ & Direct labelling with $\mathrm{SnCl}_{2}$ reduction & 536 \\
\hline Polyacrylamide NPs & Non-chelator & ${ }^{125} \mathrm{I}$ & Chloroglycoluril & 185 \\
\hline & & ${ }^{76} \mathrm{Br}$ & Chloramine-T & 185 \\
\hline Gelatin NPs & Non-chelator & ${ }^{125} \mathrm{I}$ & Iodogen tube & 537 \\
\hline & Chelator-based & ${ }^{111} \mathrm{In}$ & DTPA chelator & $538-540$ \\
\hline Polyvinyl phenol NPs & Non-chelator & ${ }^{125} \mathrm{I}$ & Iodination beads & 180 \\
\hline & & & Chloramine- $\mathrm{T}$ & 179 \\
\hline & & ${ }^{124} \mathrm{I}$ & Iodination beads & 180 \\
\hline Polydopamine NPs & Non-chelator & ${ }^{99 \mathrm{~m}_{\mathrm{Tc}}}$ & Direct labelling with $\mathrm{SnCl}_{2}$ reduction & 181 \\
\hline & & ${ }^{131} \mathrm{I}$ & Chloramine-T & 181 \\
\hline p(BAEA-co-OEGA- $c o$-VDM) & Chelator-based & ${ }^{177} \mathrm{Lu}$ & DO3A chelator with DBCO click chem & 541 \\
\hline polymeric nanostar & & ${ }^{89} \mathrm{Zr}$ & DFO & 541 \\
\hline $\begin{array}{l}\text { Poly(methacrylic acid) (PMAAc)-based } \\
\text { Single Chain Polymer NPs }\end{array}$ & Chelator-based & ${ }^{67} \mathrm{Ga}$ & DTPA & 472 \\
\hline Poly(n-butylcyano acrylate) (PBCA) NP & Non-chelator & ${ }^{99 m_{T}} \mathrm{Tc}$ & Direct labelling with $\mathrm{SnCl}_{2}$ reduction & 542 \\
\hline Melanin NPs & Non-chelator & ${ }^{64} \mathrm{Cu}$ & Direct labelling to melanin & 543 and 544 \\
\hline Silk fibroin NPs & Chelator-based & ${ }^{111} \mathrm{In}$ & DTPA & 545 \\
\hline
\end{tabular}


terminal aldehyde group (with a DO3A-hydrazine BFC) or to one of the hydroxyl groups on the cellulose backbone (via an DO3Aamine BFC, Fig. 29B). RCYs for the aldehyde-conjugated NPs after reaction with ${ }^{111}$ In was much lower (7-18\%) compared with the hydroxyl-conjugated NPs (54-65\%) likely due to lower number of chelators present. Stability in human plasma was the same for both formulations with $>90 \%$ stability over $72 \mathrm{~h}$. Ex vivo biodistribution of the two formulations in mice showed similar uptake profiles with uptake in the liver and spleen. However, vastly different amounts of uptake over all time points were observed in the lung; with $c a$. $125 \%$ ID per $g$ observed for the hydroxyl-conjugated NPs compared with ca. $12 \%$ ID per $\mathrm{g}$ for the aldehyde-conjugated NPs at $6 \mathrm{~h}$ p.i (Fig. 29B). ${ }^{33}$ This was attributed to the higher zeta-potential of the hydroxyl-conjugated NPs, and highlights once again how modification of NPs aimed at facilitating radiolabelling can lead to large differences in their properties and biodistribution in vivo.

A unique approach for chelator-based radiolabelling was reported by Benito et al. who radiolabelled single-chain poly(methacrylic acid) NPs with ${ }^{67} \mathrm{Ga} .{ }^{472}$ Interestingly, this was performed by incorporating DTPA into the polymer chain by forming an aldehyde-functionalised DTPA derivative, which was reacted with an amine groups present on a modified polymer (Fig. 29C). This modification allowed $c a$. 65\% labelling efficiencies, with $>90 \%$ stability in saline solution over $48 \mathrm{~h}$. One potential drawback of this method, however, is the potential reduction in the Ga complex stability, with only three carboxylates being available for reaction - due to the aldehyde functionalisation of the other two. Finally, Pereira et al. described the radiolabelling of PLA-based nanocapsules by the trapping of $\left[{ }^{99 \mathrm{~m}} \mathrm{Tc}\right] \mathrm{Tc}-\mathrm{HMPAO}$ during formulation of the NPs. ${ }^{533,534}$ The complex could be encapsulated with $50 \% \mathrm{LE}$, however $>30 \%$ release of the complex was observed, suggesting the trapping of $\left[{ }^{99 \mathrm{~m}} \mathrm{Tc}\right] \mathrm{Tc}-\mathrm{HMPAO}$ a sub-optimal radiolabelling method.

\subsection{Radiolabelling of inorganic nanomaterials}

4.6.1 Graphene/carbon-based nanoparticles. Graphene refers to a single layer of graphite containing stacked layers of carbon atoms in a lattice with interesting mechanical and optical properties. Nanosheets of graphene, graphene oxide (GO) and reduced graphene oxide (rGO) (Fig. 30A) have been extensively explored for use as drug carriers. ${ }^{546}$ Similarly, carbon nanotubes (CNTs) - also derived from graphite - have also been investigated for the drug delivery of small molecules. ${ }^{547}$ As well as their use for drug delivery platforms, graphene-based nanomaterials and carbon nanotubes are also of high interest for biomedical imaging. ${ }^{548,549}$ Whilst this is primarily due to their interesting optical properties, the radiolabelling of these nano platforms has also been explored. Table 14 summarises the techniques used to radiolabel graphene/carbon-based NPs for in vivo imaging with radionuclide imaging.

Several groups have reported the radiolabelling of nanographene oxide sheets with ${ }^{125}$ I using the chloramine-T method with RCYs of $c a$. 50-60\%, with high in vitro serum and in vivo stability. ${ }^{163,164,552}$ Radioiodination was suggested to occur at the edges of the graphene sheets where defects exist. The nonchelator labelling of different types of graphene-based NPs with various radiometals has also been reported (Table 14). Zhan and co-workers radiolabelled multi-walled carbon nanotubes (MWCNTs) directly with ${ }^{99 \mathrm{~m}_{\mathrm{Tc}}}$ after $\mathrm{SnCl}_{2}$ reduction resulting in ca. $90 \% \mathrm{RCY}^{553,554}$ Direct labelling of graphene oxide nanomaterials with ${ }^{99 \mathrm{~m}} \mathrm{Tc}$ has also been reported by several groups. ${ }^{555,556,559}$ Zhang et al. also used this direct labelling method for carbon NPs with ${ }^{188}$ Re. ${ }^{560-562}$ A slightly modified method was developed by Cao et al. who produced the

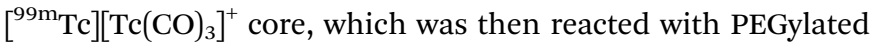
nanographene oxide. ${ }^{559}$ Radiolabelling peaked at $80 \%$ RCY after $5 \mathrm{~min}$, but rapidly declined at later timepoints. The authors reported this as being due to reduction of carbonyl and hydroxyl groups on the GO layer by $\mathrm{NaBH}_{4}$ used to produce the $\left[{ }^{99 \mathrm{~m}} \mathrm{Tc}\right]\left[\mathrm{Tc}(\mathrm{CO})_{3}\right]^{+}$core. This was confirmed by the low labelling $(8 \%)$ of $\mathrm{GO}$ reduced with $\mathrm{NaBH}_{4} \cdot{ }^{559}$ However, this may also be due to the lack of appropriate binding sites on $\mathrm{GO} / \mathrm{rGO}$ for $\left[{ }^{99 \mathrm{~m}} \mathrm{Tc}\right]\left[\mathrm{Tc}(\mathrm{CO})_{3}\right]^{+}$which prefers multidentate ligands, often containing aromatic amines. ${ }^{586}$ This work suggests this method may be inappropriate for use with GO NPs.

As well as with Tc/Re, chelate-free labelling with other radiometals is also possible (Fig. 30A). Shi et al. reported a method to directly label GO and reduced GO with ${ }^{64} \mathrm{Cu}^{2+} \cdot 550$ The binding of the copper ion was proposed to occur via an interaction with the $\pi$ bond electrons on the graphene surface (Fig. 30A and B). This was supported by the increased RCY seen with reduced GO (60\% RCY compared with ca. $20 \%$ for GO) which has higher abundance of $\pi$ electrons. RGO also demonstrated higher serum stability with $c a$. $80 \%$ remaining on the GO after incubation in mouse serum for $24 \mathrm{~h}$ compared with ca. $50 \%$ for GO. ${ }^{550}$ Sarpaki et al. also reported that ${ }^{68} \mathrm{Ga}^{3+}$ could also be attached to GO in a chelate-free method. This was again proposed to be based on interactions with the $\pi$ bond electrons and also binding to oxygen donors on the GO surface. This interaction was shown to be highly favourable with RCYs and stability in human serum (up to $2 \mathrm{~h}$ ) both $>95 \%$. ${ }^{551}$ The authors also reported a novel bis(semithiocarbazonate) ${ }^{68} \mathrm{Ga}^{3+}$ complex (Fig. 30C) capable of radiolabelling GO. Radiolabelling with this complex allowed high RCY $>95 \%$ and high serum stability $>95 \%$ up to $2 \mathrm{~h}^{551}$ Characterisation using energydispersive X-ray spectroscopy (EDX) mapping the non-radioactive gallium complex suggested that the complex was incorporated non-covalently within the GO layers (Fig. 30C).

Several groups have used chelator-based methods for labelling graphene-based nanomaterials (Table 14); with a large number of reports using the chelator DTPA with ${ }^{111}$ In. ${ }^{566-572}$ Al-Jamal and collaborators reported RCYs varying between 8.0-85\% with conjugation of the chelator occurring through one of the carboxylate groups on EDTA, ${ }^{566,567,569,571,572}$ whereas Zhang reported higher yields (up to 95\%) when using an extended DTPA compound with an additional carboxylate for conjugation when using similar reaction conditions. ${ }^{570}$ Interestingly, Cornelissen et al. found that $\pi$-bond interactions between GO and the benzene ring (Fig. 30A) on the p-SCN-Bn-DTPA 

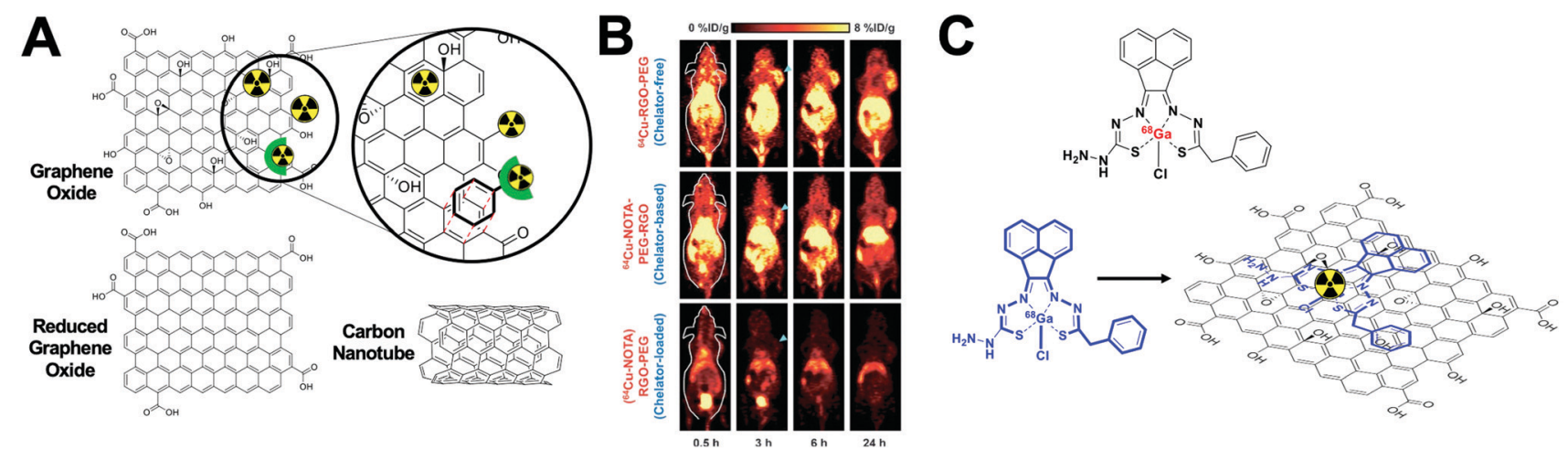

Fig. 30 (A) Structures of graphene oxide (GO), reduced graphene oxide ( $\mathrm{rGO}$ ) and carbon nanotubes (CNTs) along with a schematic of the various ways to radiolabel these carbon-based structures. Radiometals and radiohalogens can bind directly to the graphene structures and chelators can be attached to the nanomaterials either via conjugation or by $\pi-\pi$ interactions between BFCs and the graphene structures. (B) Representative PET images of graphene-oxide nanosheets radiolabelled with ${ }^{64} \mathrm{Cu}$ in $4 \mathrm{~T} 1$ tumor-bearing mice. Adapted from Shi et al. ${ }^{550}$ (C) Structure of a novel bissemithiocarbazonate ${ }^{68} \mathrm{Ga}^{3+}$ complex for radiolabelling graphene oxide NPs. (top) and a schematic showing the proposed incorporation of the ${ }^{68} \mathrm{Ga}{ }^{3+}$ complex into the GO sheets (bottom). Reported by Sarpaki et al. ${ }^{551}$

Table 14 Table summarising the methods, radioisotopes and techniques employed to radiolabel graphene/carbon-based nanoparticles

\begin{tabular}{|c|c|c|c|}
\hline Radiolabelling method & Radionuclide & Radiolabelling mediator & Ref. \\
\hline & ${ }^{131} \mathrm{I}$ & Chloramine-T & 165 \\
\hline & $99 \mathrm{~m}_{\mathrm{Tc}}$ & Direct labelling with $\mathrm{SnCl}_{2}$ reduction & 553-558 \\
\hline & ${ }^{188} \mathrm{Re}$ & Direct labelling with $\mathrm{SnCl}_{2}$ reduction & $560-562$ \\
\hline & ${ }^{64} \mathrm{Cu}$ & Direct labelling & 550 \\
\hline & ${ }^{68} \mathrm{Ga}$ & Direct labelling & 551 \\
\hline & & DTPA chelator & $566-572$ \\
\hline & ${ }^{99 \mathrm{~m}_{\mathrm{Tc}}}$ & DOTA chelator & 573 \\
\hline & ${ }^{64} \mathrm{Cu}$ & HPPH (porphyin PDT agent) chelator & 574 \\
\hline & & DO3A chelator & $575-577$ \\
\hline & & NOTA chelator & 550,578 and 579 \\
\hline & & DOTAM chelator & 580 \\
\hline & ${ }^{66} \mathrm{Ga}$ & NOTA chelator & 581 \\
\hline & ${ }^{89} \mathrm{Zr}$ & DFO chelator & 583 \\
\hline & ${ }^{225} \mathrm{Ac}$ & DOTA chelator & 583 \\
\hline & ${ }^{57} \mathrm{Co}$ & $\mathrm{MeAMN}_{3} \mathrm{~S}_{3}$ sar & 584 \\
\hline & ${ }^{177} \mathrm{Lu}$ & DOTA chelator & 585 \\
\hline
\end{tabular}

bifunctional chelator, could be strong enough to allow of the GO labelling with ${ }^{111}$ In. ${ }^{568}$ RCYs were high (>99\%), with 95\% serum stability up to $24 \mathrm{~h}$. However, bladder uptake seen in early $(1 \mathrm{~h}$ p.i.) SPECT/CT images suggests some of the ${ }^{111}$ In-DTPA is released from the GO and excreted. ${ }^{568}$ Similarly, Shi et al. reported that the p-SCN-Bn-NOTA bifunctional chelator also be non-specifically loaded onto RGO; which was hypothesised to be either from hydrophobic interactions or $\pi$-bond interactions between the RGO and the benzene ring on the. ${ }^{550}$ RGO loaded and conjugated with NOTA both showed high RCYs of $c a .90 \%$ with ${ }^{64} \mathrm{Cu}$. However, the NOTA-loaded RGO showed lower serum stability with only ca. $50 \%$ remaining after incubation in mouse serum for $24 \mathrm{~h}$ compared with $>80 \%$ for the NOTA-conjugated RGO. Similar to the study by Cornelissen et al. in vivo bladder uptake for the NOTA-loaded RGO was observed suggesting the release and excretion of ${ }^{64} \mathrm{Cu}$-NOTA occurred (Fig. 30B). ${ }^{550}$

4.6.2 Iron oxide nanoparticles (IONPs). Iron oxide nanoparticles (IONPs) are well-established $T_{2}$ (negative) contrast agents for MRI and hyperthermia therapy. In the last few years, several radiolabelled formulations have been also developed expanding their application to multimodal imaging and therapy. ${ }^{625,626}$ A wide variety of radiolabelling mediators and methods have been described that allow the tagging IONPs with many different radionuclides (Table 15). Therefore, due to this high diversity, it is difficult to define one method as the 'gold standard' for effective and robust radiolabelling. However, several 
Table 15 Table summarising the methods, radioisotopes and techniques employed to radiolabel iron-oxide nanoparticles

\begin{tabular}{|c|c|c|c|}
\hline Radiolabelling method & Radionuclide & Radiolabelling mediator & Ref. \\
\hline Chelator-based & $\begin{array}{l}{ }^{89} \mathrm{Zr} \\
{ }^{18} \mathrm{~F} \\
{ }^{99} \mathrm{~m}_{\mathrm{Tc}}\end{array}$ & $\begin{array}{l}\text { DOTA } \\
\text { NOTA } \\
\text { Bis(dithiocarbamate)bisphosphonate } \\
\text { Thiosemicarbazone } \\
\text { DOTA/NOTA } \\
\text { DFO } \\
{ }_{18} \text { F-AlF/NOTA } \\
\text { Polyacrylic acid } \\
\text { Bisphosphonate derivatives } \\
\text { DMSA } \\
\text { Lipoic acid based ligands } \\
\text { DTPA } \\
\text { PCTA } \\
\text { DOTA } \\
\text { Polyacrylic acid } \\
\text { Imidodiphosphate (IDP) or Inositol hexaphosphate (IHP) } \\
\text { PEG600 diacid } \\
\mathrm{N}_{2} \mathrm{~S}_{4} \\
\text { (Z)-2-Methoxyimino-2-(2-aminothiazol-4-yl)-acetic acid } \\
\text { SnCl }_{2} \text { reduction } \\
\text { Polyacrylic acid }\end{array}$ & $\begin{array}{l}587 \text { and } 588 \\
589-591 \\
592 \\
593 \\
594-596 \\
597 \\
598 \\
599 \\
600-603 \\
604 \text { and } 605 \\
606 \\
607-609 \\
116 \\
610 \\
599 \\
611 \\
612 \\
613 \\
614 \\
615 \\
599\end{array}$ \\
\hline Non-chelator & $\begin{array}{l}{ }^{64} \mathrm{Cu} \\
{ }^{68} \mathrm{Ga} /{ }^{67} \mathrm{Ga} \\
{ }^{89} \mathrm{Zr} \\
{ }^{11} \mathrm{C} \\
{ }^{18} \mathrm{~F} \\
{ }^{69} \mathrm{Ge} \\
{ }^{59} \mathrm{Fe} \\
{ }^{*} \mathrm{As} \\
{ }^{99 \mathrm{~m}} \mathrm{Tc} \\
{ }^{111} \mathrm{In} \\
{ }^{125} \mathrm{I} \\
{ }^{90} \mathrm{Y} \\
{ }^{177} \mathrm{Lu} \\
{ }^{223} \mathrm{Ra} \\
{ }^{225} \mathrm{Ac}\end{array}$ & $\begin{array}{l}\text { Chemical adsorption } \\
\text { Hot }+ \text { cold precursors } \\
\text { Chemical adsorption } \\
\text { Hot }+ \text { cold precursors } \\
\text { Chemical adsorption } \\
{\left[{ }^{11} \mathrm{C}\right] \text { methyl iodide }} \\
{ }^{18} \mathrm{FDG} / \text { chemoselective oxime formation } \\
\text { Chemical adsorption/Al }(\mathrm{OH})_{3} \\
\text { Chemical adsorption } \\
\text { Hot + cold precursors } \\
\text { Core-doped/post-synthetic method } \\
\text { Chemical adsorption } \\
\text { SnCl }{ }_{2} \text { reduction } \\
\text { Chemical adsorption } \\
\text { Cold + hot precursors } \\
\text { Chloramine-T } \\
\text { Bolton-Hunter reagent } \\
\text { Chemical adsorption } \\
\text { Physisorption } \\
\text { Chemical adsorption } \\
\text { Chemical adsorption } \\
\text { Hot + cold precursors }\end{array}$ & $\begin{array}{l}616 \\
117 \\
166,592 \text { and } 617 \\
115 \\
209,603,618 \text { and } 619 \\
132 \\
197 \\
213-215 \\
620 \\
109-111 \\
621 \\
208 \\
622 \text { and } 623 \\
618 \\
119 \\
140 \text { and } 141 \\
142 \\
616 \\
612 \\
616 \\
624 \\
118\end{array}$ \\
\hline
\end{tabular}

examples providing high RCY, RCP and RCS for both chelatorbased and non-chelator methods are summarised below.

Initially, the main strategies for the radiolabelling of IONPs were based on chelating agents such as DTPA, DOTA and NOTA attached to the NP surface. ${ }^{625,627}$ In 2008, Jarret et al. introduced DOTA as a chelating agent for IONPs. ${ }^{587}$ Although with moderate RCY (up to $21 \%$ ), this work revealed the benefit of incorporating ${ }^{64} \mathrm{Cu}$ into DOTA before the conjugation with the NPs as well as the better performance of $p$-SCN-Bz-DOTA over $p-\mathrm{NH}_{2}$-Bz-DOTA during the conjugation - likely due to the decrease in the steric hindrance. Lee et al. reported a similar strategy not only for the ${ }^{64} \mathrm{Cu}$ radiolabelling with DOTA but also for the conjugation with an RGD peptide through a PEGmaleimide linker (Fig. 31A). This formulation demonstrated high affinity towards integrins due to the RGD peptide allowing angiogenesis-targeted tumour PET/MRI detection (Fig. 31B). ${ }^{588}$ Among other chelating agents, bisphosphonate-based bifunctional chelators offer a versatile strategy for the radiolabelling of SPIOs with PET or SPECT radionuclides via direct binding to the $\mathrm{Fe}_{3} \mathrm{O}_{4}$ surface. Bisphosphonates have shown excellent properties as anchors to functionalise iron oxide and other nanomaterials based on metal oxides and calcium phosphates. For instance, bis(dithiocarbamatebisphosphonate) (dtcbp) was introduced as a chelating agent for ${ }^{64} \mathrm{Cu}$ with instantaneous and quantitative complexation at room temperature. In combination with IONPs for $15 \mathrm{~min}$ at $100{ }^{\circ} \mathrm{C}$, the attachment of $\left[{ }^{64} \mathrm{Cu}\right]\left[\mathrm{Cu}(\mathrm{dtcbp})_{2}\right]$ provided ${ }^{64} \mathrm{Cu}$-labeled IONPs with 95\% RCY, 100\% RCP and quantitative RCS after $48 \mathrm{~h}$ of incubation with human serum at $37{ }^{\circ} \mathrm{C}$, most likely a result of the protective action of the dextran coating. ${ }^{628}$ IONPs were also successfully PEGylated with high surface density and radiolabelled with ${ }^{99} \mathrm{~m}$ Tc using bisphosphonate-functionalised PEG conjugates and dipicolylamine (DPA)-alendronate. ${ }^{600,601}$ The biodistribution of the PEG-bisphosphonate functionalised IONPs radiolabelled with ${ }^{99 \mathrm{~m}}$ Tc (Fig. 31C) was studied by SPECT/CT showing long circulation times, as expected 
A
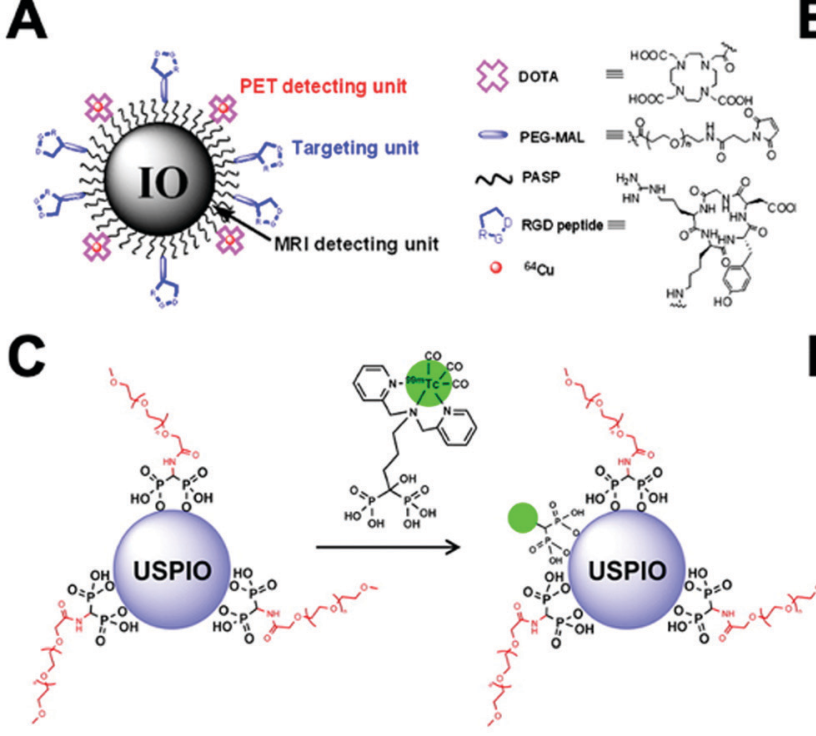

E

MRI detectable

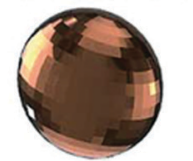

$\mathrm{Fe}_{3} \mathrm{O}_{4}$ or $\gamma-\mathrm{Fe}_{2} \mathrm{O}_{3}$ nanoparticle core

B

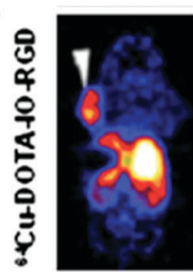

$1 \mathrm{~h}$

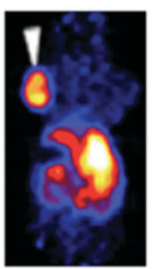

4h

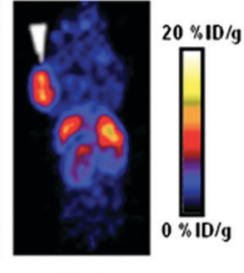

$21 \mathrm{~h}$

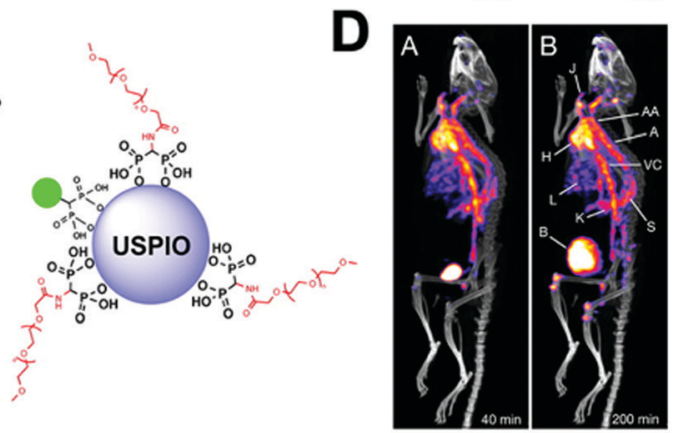

F
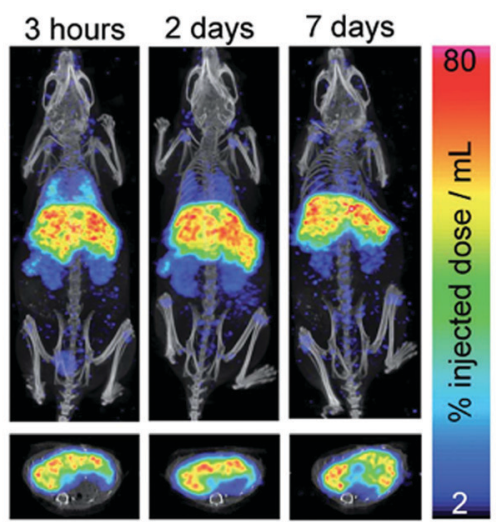

Fig. 31 (A) Illustration of the ${ }^{64} \mathrm{Cu}$-DOTA-IO-RGD PET/MRI probe, (B) decay-corrected wholebody coronal PET images of nude mouse bearing human U87MG tumor at 1,4 , and $21 \mathrm{~h}$ after injection of $3.7 \mathrm{MBq}$ of ${ }^{64} \mathrm{Cu}$-DOTA-IO-RGD, adapted with permission from ref. 588. (C) Radiolabeling of PEG(5)-BPUSPIOs with the radiolabelled bisphosphonate ${ }^{99 m}$ Tc-DPA-ale, (D) in vivo SPECT-CT studies with PEG(5)-BP-USPIO: (A and B) maximum intensity projection SPECT-CT images after i.v. injection of radiolabeled $\left({ }^{99 \mathrm{~m}} \mathrm{TC}\right) \mathrm{PEG}(5)-\mathrm{BP}-\mathrm{USPIO}$ at the first (A, $\left.40 \mathrm{~min}\right)$ and last (B, $\left.200 \mathrm{~min}\right)$ time points (labels: $\mathrm{H}=$ heart, $\mathrm{J}=$ jugular vein, $\mathrm{AA}=$ aortic arch, $\mathrm{A}=$ aorta, $\mathrm{VC}=$ vena cava, $\mathrm{L}=$ liver, $\mathrm{K}=$ kidney, $\mathrm{S}=$ spleen, $\mathrm{B}=$ bladder), adapted with permission from ref. 601, (E) synthesis of radiolabelled IONPs using radiometal chloride salts $\left(\mathrm{MCl}_{n}\right)$ to form an oxidised radiometal coating, $(\mathrm{F})$ maximum intensity projection ${ }^{111}$ In SPECT/CT at $3 \mathrm{~h}, 2$ and $7 \mathrm{~d}$ post-injection confirms presence of labelled iron oxides in the liver, lung, kidneys, and spleen of C57BL/ 6 mice. Corresponding axial slices (bottom) show co-localisation of the radiolabelled IONPs and the liver, adapted with permission from ref. 618.

from the high density of PEG, and high signal in the vasculature of Balb/C mice even at $200 \mathrm{~min}$ post i.v. injection (Fig. 31D).

Within the non-chelator based radiolabelling strategies described in Section 4.4, chemical adsorption and hot + cold precursors are the most widely reported methods to radiolabel of IONPs. In the chemical adsorption category, reported radiolabelling protocols showed the high affinity of the magnetite $\left(\mathrm{Fe}_{3} \mathrm{O}_{4}\right)$ and maghemite $\left(\gamma-\mathrm{Fe}_{2} \mathrm{O}_{3}\right)$ surface towards different metallic radionuclides as described in detail in Section 4.4.5. Recently, Patrick et al. introduced the concept of radiomineralisation (SRM) to explain this affinity. ${ }^{618}$ In this work, ${ }^{111}$ In and ${ }^{89} \mathrm{Zr}$ magnetite/maghemite NPs were synthesised by heat-induced chemical adsorption demonstrating the deposition of radionuclide metal oxides onto the surface of the IONPs (Fig. 31E). The reactions conducted at $90{ }^{\circ} \mathrm{C}$ and $\mathrm{pH}=9$ for
90 min provided RCYs between $79-85 \%$ for ${ }^{111}$ In-IONPs and 94\% for ${ }^{89} \mathrm{Zr}$-IONPs. SPECT/CT biodistribution studies of ${ }^{111} \mathrm{In}$-IONPS in C57BL/6 mice showed main accumulation in liver and spleen with significant accumulation in the lungs in the first 3 hours post-injection (Fig. 31F).

The hot + cold precursors methodology has also been used for the integration of PET, SPECT and therapeutic radionuclides into the core of NPs. Dextran-coated IONPs doped with ${ }^{64} \mathrm{Cu}$ or ${ }^{68} \mathrm{Ga}$ were developed by fast microwave-driven protocols. ${ }^{115,117}$ Using $\mathrm{FeCl}_{3} \cdot 6 \mathrm{H}_{2} \mathrm{O}$, dextran and $\left[{ }^{68} \mathrm{Ga}^{-} \mathrm{GaCl}_{3}\right.$ as starting reagents, a $10 \mathrm{~min}$ microwave protocol in water provided ${ }^{68} \mathrm{Ga}$ doped IONPs with RCY greater than 90\%, 100\% RCP and quantitative RCS under different physiological media. The microwave synthesis was also successful when using citric acid as a coating (instead of dextran), demonstrating the versatility of this IONP radiolabelling method. ${ }^{629,630}$ 
4.6.3 Silica nanoparticles. Silica nanoparticles $\left(\mathrm{SiO}_{2} \mathrm{NPs}\right)$ are defined by an intrinsically high particle stability that, in combination with a low toxicity profile, makes them a suitable nanoplatform with multiple biomedical applications. ${ }^{631}$ Additionally, the possibility of developing mesoporous nanomaterials with precise control over the pore size and shape with bio-responsive gating properties has led to the wide use of these NPs for controlled drug delivery applications. ${ }^{632,633}$ Hence, the radiolabelling of $\mathrm{SiO}_{2}$ NPs to study their biodistribution and pharmacokinetics is highly valuable.

Within the different radiolabelling methods for $\mathrm{SiO}_{2} \mathrm{NPs}$ (Table 16), the chemical adsorption of radiometals appears to be the best choice due to its facile, fast and stable radiolabelling. Shaffer et al. evaluated the incorporation of ${ }^{68} \mathrm{Ga}$, ${ }^{64} \mathrm{Cu},{ }^{89} \mathrm{Zr},{ }^{90} \mathrm{Y},{ }^{111} \mathrm{In}$ and ${ }^{177} \mathrm{Lu}$ into amorphous silica NPs at different temperatures, $\mathrm{pH}$ and reaction times. ${ }^{211}$ The results showed RCYs $>99 \%$ with all radionuclides when radiolabelling is conducted at $70{ }^{\circ} \mathrm{C}$ and $\mathrm{pH}=7.3$ between $15 \mathrm{~min}$ and $1 \mathrm{~h}$ of incubation (Fig. 32A). No significant changes over the RCYs were observed at different $\mathrm{pH}$ whilst the RCY greatly increased with the temperature. Additionally, a competitive chelation protocol with EDTA was carried out showing stable radiolabelling only in samples heated at $70{ }^{\circ} \mathrm{C}$. This suggests that high temperatures are needed to reach the required binding activation energy - rather than increase the radionuclide diffusion through the $\mathrm{SiO}_{2}$ NP. The RCS was also evaluated in $50 \%$ fetal bovine serum showing a clear relationship between the oxophilicity of the radionuclide and the RCS (Fig. 32A). This clearly highlights the affinity of the radionuclides towards the oxygenrich matrix of the $\mathrm{SiO}_{2}$ NP. For 'softer' radiometals, such as ${ }^{64} \mathrm{Cu}(\mathrm{II})$, the stability was very poor with $50 \%$ of radionuclide leaching after just $4 \mathrm{~h}$ (Fig. 32A). In vivo PET imaging demonstrated high RCS of ${ }^{68} \mathrm{Ga}$ and ${ }^{89} \mathrm{Zr} \mathrm{SiO}_{2}$ NPs by showing high uptake in the elimination organs (liver and spleen), and different profiles compared to their respective free radionuclides (Fig. 32B and C). Interestingly, Cheng et al. compared this methodology for the ${ }^{89} \mathrm{Zr}$ radiolabelling of ultrasmall cRGDY-conjugated fluorescent silica NPs ( $C^{\prime}$ dots) with the radiolabelling through a chelator-based protocol using DFO as chelating agent (Fig. 32D). ${ }^{34}$ Both NPs, with an average size of 6-7 nm were successfully radiolabelled with high RCY. To evaluate differences in RCS, both formulations were incubated in human serum at $37{ }^{\circ} \mathrm{C}$ obtaining high RCS of $>99 \%$ in both cases. RCS and circulations half-lives were also studied by injecting both NPs in nude mice, finding a higher degradation of the non-chelator NPs $(>25 \%)$ than chelator-based NPs $(<2 \%) 48 \mathrm{~h}$ post-injection with similar circulation times of around $15 \mathrm{~h}$. Biodistribution of both NPs was evaluated by dynamic PET during $60 \mathrm{~min}$ after the injection. Both formulations showed a similar trend with intense signal assigned to the circulation and most interestingly, the renal clearance due to the small size of the particles (Fig. 32E). Further ex vivo biodistribution studies revealed higher bone uptake in the non-chelator formulation attributed to the ${ }^{89} \mathrm{Zr}$ detachment in agreement with the lower stability previously observed.

As well as the above mentioned radionuclides, this strategy has also been evaluated to attach different radioarsenic isotopes, ${ }^{*}$ As $\left.{ }^{*}=72,76,74,71\right)$ and ${ }^{45} \mathrm{Ti}$ demonstrating the high versatility of this radiolabelling method. ${ }^{650,651}$ Burke et al. used this strategy for the radiolabelling of iron oxide nanorods coated with silica. In this work, a series of nanorods conjugated with siloxane terminated DO3A chelator and a siloxane polyethylene glycol (PEG) derivative were radiolabelled with ${ }^{68} \mathrm{Ga}$ at $90{ }^{\circ} \mathrm{C}$ for $15 \mathrm{~min}$. The results showed quantitative RCY and $95 \%$ of RCS in human serum at $37{ }^{\circ} \mathrm{C}$ for $3 \mathrm{~h}$ in the sample without DO3A chelator showing the high affinity of the silica layer towards ${ }^{68} \mathrm{Ga} .{ }^{646}$

4.6.4 Gold nanoparticles. Gold NPs are arguably one of the most popular materials in nanotechnology. The excellent

Table 16 Table summarising the methods, radioisotopes and techniques employed to radiolabel silica nanoparticles

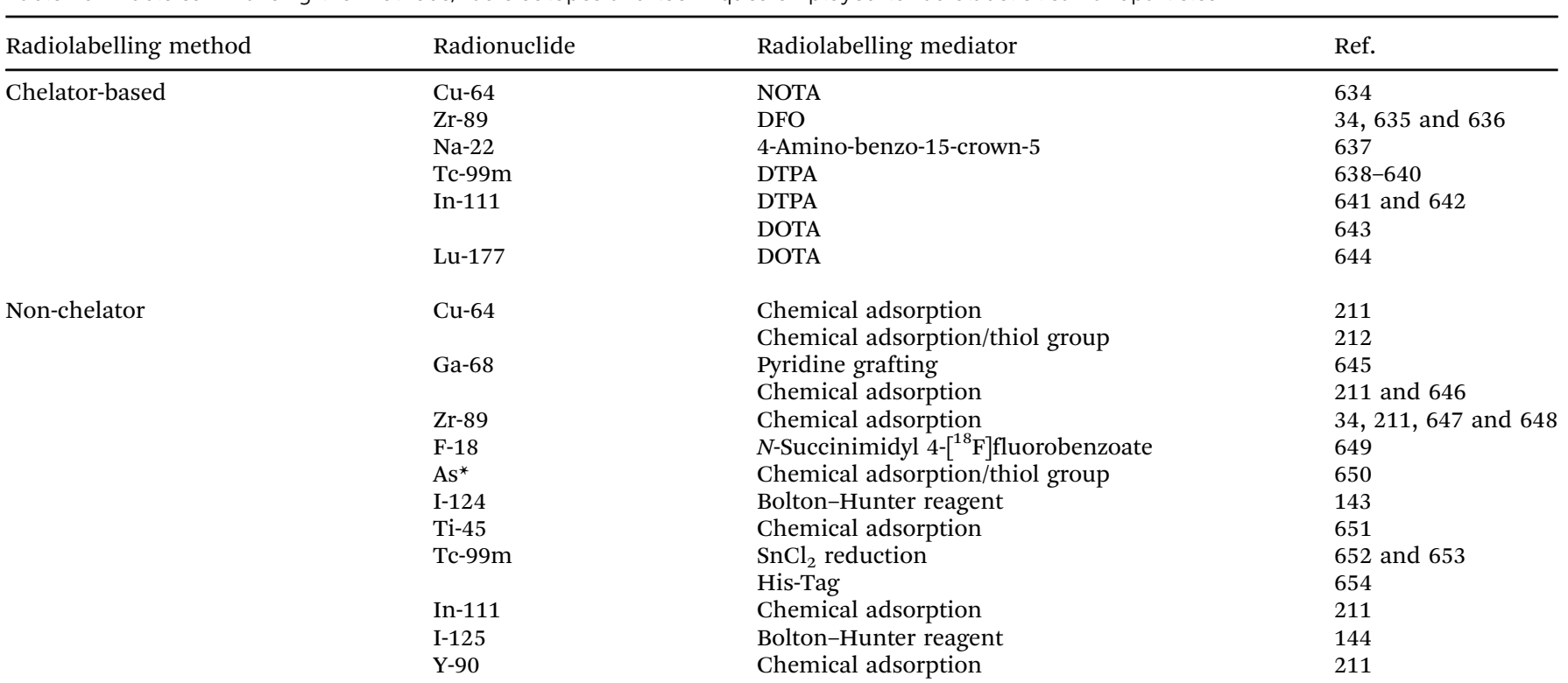


A

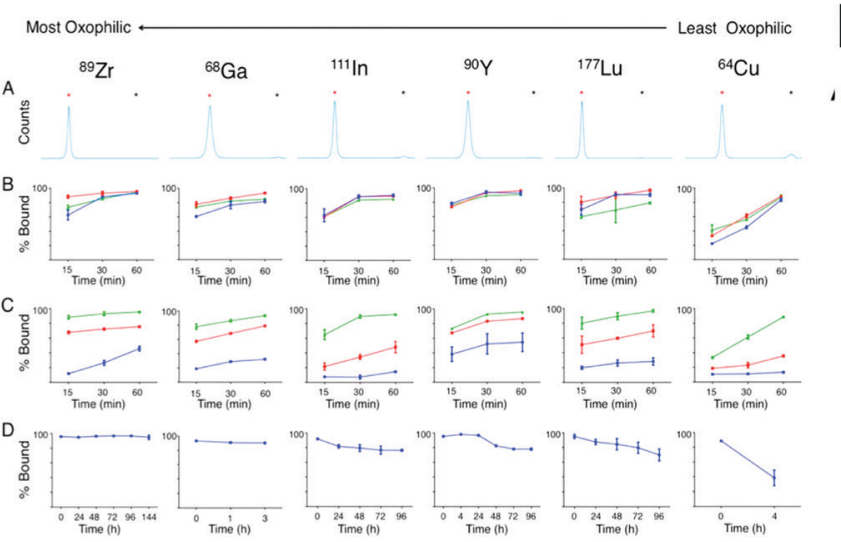

B

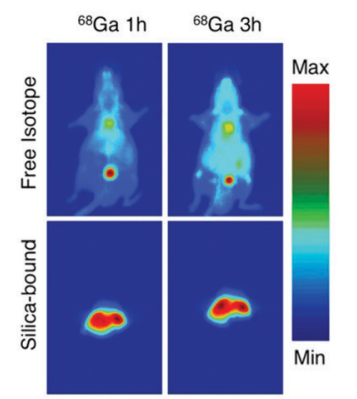

C

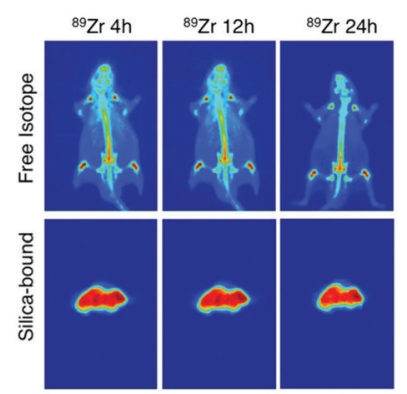

D

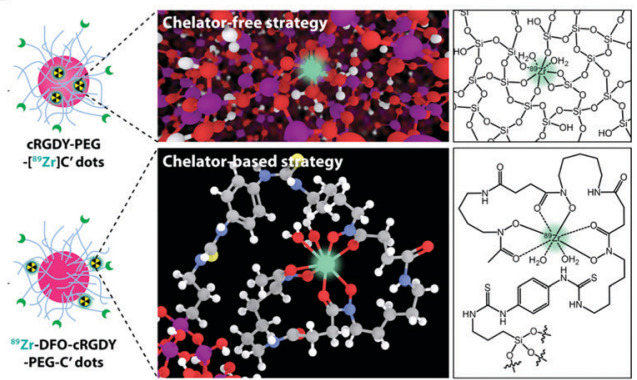

E

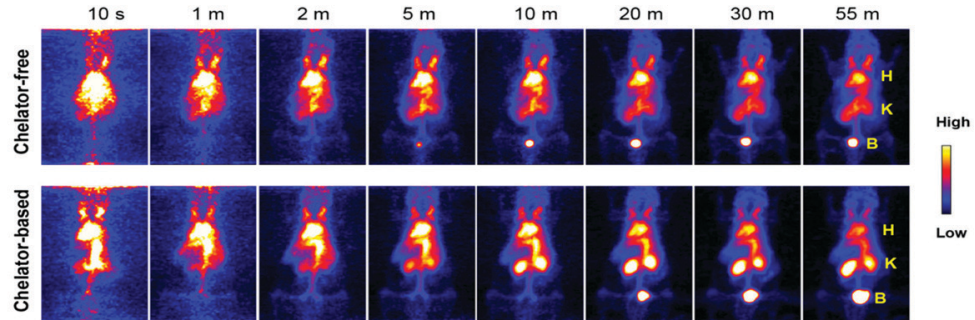

Fig. 32 (A) Radiolabelling and serum stability of silica nanoparticles: (a) instant thin-layer chromatographs of radiolabelled silica nanoparticles. The red asterisk denotes the origin, where the nanoparticles remain, and the black asterisk denotes the solvent front, where the free activity would be located. Controls of buffer-only solutions (no particles) were performed with each condition with $>95 \%$ signal at the free activity peak. (b) Percent radioisotope bound to silica nanoparticles as a function of time and $\mathrm{pH}$. The blue, red, and green lines indicate radiolabelling at $\mathrm{pH}=5.5,7.3$, and 8.8 , respectively. (c) Percent radioisotope bound to silica nanoparticles as a function of time and temperature. The blue, red, and green lines indicate radiolabelling at 4,37 , and $70{ }^{\circ} \mathrm{C}$, respectively. (d) Serum stability of silica nanoparticles radiolabelled at $\mathrm{pH}=7.3$ and $70{ }^{\circ} \mathrm{C}$, then incubated in $50 \%$ FBS at $37{ }^{\circ} \mathrm{C}$. (B) In vivo coronal PET maximum intensity projections (MIPs) of free (top) and silica-bound (bottom) ${ }^{68} \mathrm{Ga}$ at 1 and $3 \mathrm{~h}$ post injection in athymic nude mice. (C) In vivo coronal PET maximum intensity projections (MIPs) of free (top) and silica-bound (bottom) ${ }^{89} \mathrm{Zr}$ at 1 and $3 \mathrm{~h}$ post injection in athymic nude mice, (D) schematic representation of chelator-free CRGDY-PEG- $\left[{ }^{89} \mathrm{Zr}\right] \mathrm{C}^{\prime}$ dots and chelator-based ${ }^{89} \mathrm{Zr}$-DFO-cRGDY-PEG-C' dots, (E) comparison of dynamic PET imaging results in mice for chelator-free ${ }^{89} \mathrm{Zr}$-labeled CRGDY-PEG-C' dots and chelator-based ${ }^{89} \mathrm{Zr}$-labeled $\mathrm{CRGDY}$-PEG-C' dots. $\mathrm{H}$ : heart; K: kidney; B: bladder. Adapted and reproduced with permission from ref. 34 and 211.

physicochemical, optical, and photoacoustic contrast properties, coupled with their high biocompatibility, have made gold NPs a prime candidate for several applications in nanomedicine. ${ }^{655}$ The preparation of particles with different shapes and morphologies, such as nanocages, nanoshells, nanorods or nanospheres in a straightforward and controllable way, have led to a wide variety of radiolabelled gold NPs for PET, SPECT and radiotherapy applications. ${ }^{656-658}$

Similar to IONPs, it is difficult to highlight the most appropriate radiolabelling method for gold NPs due to the diversity of reported examples (Table 17) and each formulation should be considered individually. However, radiolabelling with ${ }^{64} \mathrm{Cu}$ by both, chelator-based and chelator free methods and with ${ }^{99 \mathrm{~m}} \mathrm{Tc}$ by chelator-based protocols make up a large portion of the literature. Moreover, due to the availability of different $\mathrm{Au}$ radionuclides, the radiolabelling by hot + cold precursors to incorporate ${ }^{195} \mathrm{Au},{ }^{198} \mathrm{Au}$, or ${ }^{199} \mathrm{Au}$ radionuclides into the crystal lattice of the particle is also a suitable strategy - as described in Section 4.4.1. Several radiolabelling strategies for gold NPs are based on the highly stable bonds that gold forms with sulfur. Campbell et al. reported the covalent attachment of thiolated gold NPs with DOTA-maleimide with further radiolabelling at different $\mathrm{pH}$ and temperatures (Fig. 33A). A high RCY of 96\% was observed when the $\mathrm{pH}$ is increased from 5.5 to 8.8 without significant variations between room temperature and $60{ }^{\circ} \mathrm{C}$ reactions. RCS was also high at these conditions with a leaching of $4 \%$ of ${ }^{64} \mathrm{Cu}$ after 24 hours of incubation in EDTA. ${ }^{659}$ PET/CT studies showed a rapid accumulation in liver and spleen when particles are administered intravenously. However, after oral administration, the images revealed a different pattern with initial accumulation in stomach and further uptake in small intestine, cecum, and large intestine with no presence of NPs after $24 \mathrm{~h}$ (Fig. 33B). Within the non-chelator strategies, a highly interesting report by Sun $e t$ al. described the reduction of ${ }^{64} \mathrm{Cu}^{2+}$ over the surface of different PEGylated gold nanomaterials (Fig. 33C). ${ }^{660}$ In this radiolabelling reaction, ${ }^{64} \mathrm{Cu}^{2+}$ is first reduced in hydrazine and exposed to the gold PEGylated nanomaterial in the presence of poly(acrylic acid) at room temperature. These conditions provided RCYs of near $100 \%$ after $1 \mathrm{~h}$ for gold NPs of different sizes (10, 20 and $80 \mathrm{~nm})$ and most interestingly, with different shapes - such as spheres, rods and hexapods. In the absence of hydrazine, a decrease of 
Table 17 Table summarising the methods, radioisotopes and techniques employed to radiolabel gold nanoparticles

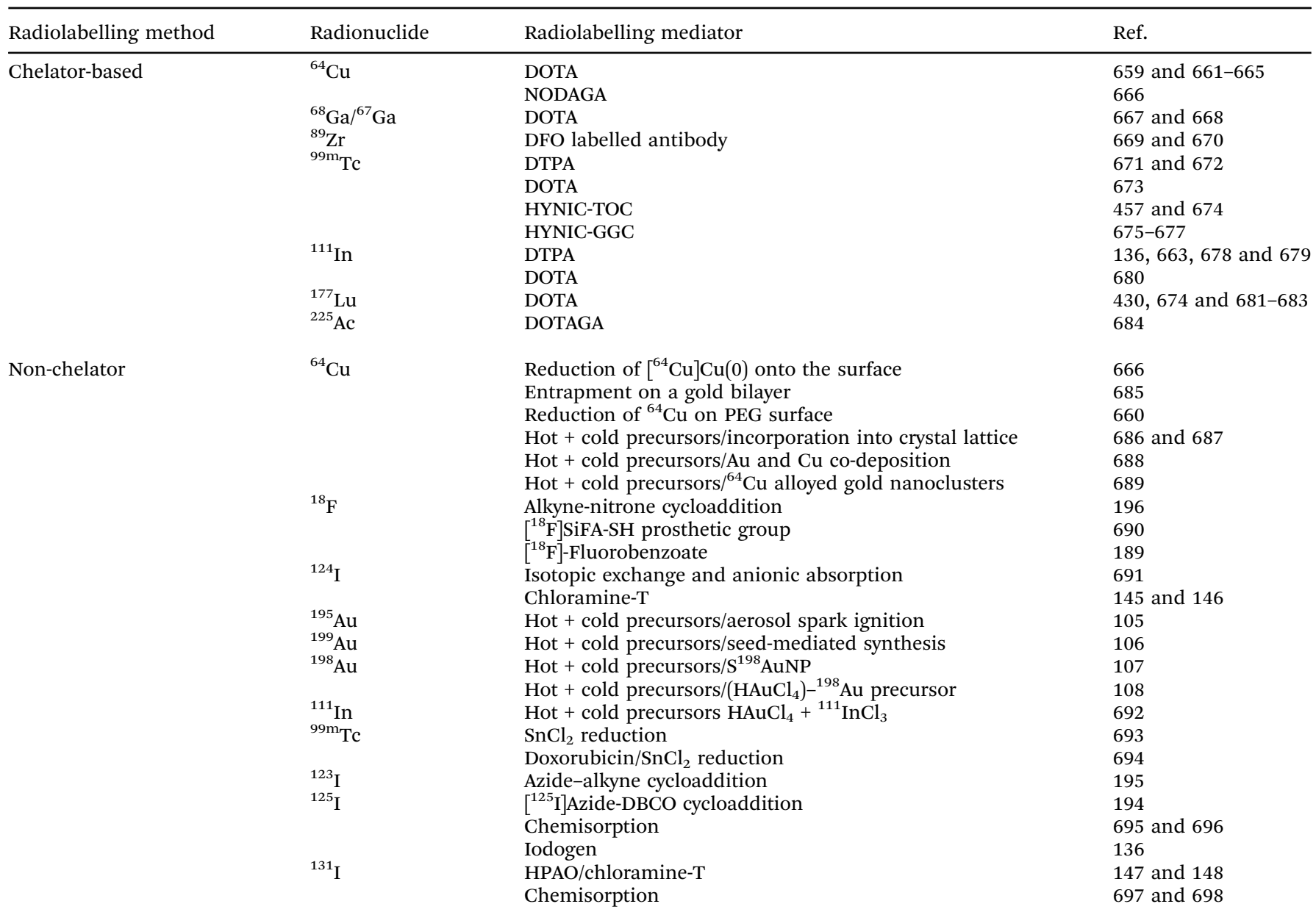

the RCY to $30 \%$ was observed, highlighting the key role of the reducing agent. The RCS was also evaluated; with a $3 \%$ of ${ }^{64} \mathrm{Cu}$ release after the incubation of the NPs for $24 \mathrm{~h}$ in PBS, further confirmed in vivo after an imaging biodistribution study showing different liver uptake profiles (Fig. 33D). Consequently, this technique clearly represents a highly versatile non-chelator method for the radiolabelling of AuNPs with ${ }^{64} \mathrm{Cu}$.

The hot + cold precursors method has also been extensively used not only for the radiolabelling with ${ }^{64} \mathrm{Cu}$, but also with ${ }^{111}$ In and different $\mathrm{Au}$ isotopes $\left({ }^{195} \mathrm{Au},{ }^{198} \mathrm{Au}\right.$ and $\left.{ }^{199} \mathrm{Au}\right) . \mathrm{Ng}$ et al. synthesised Au NPs doped with ${ }^{111}$ In with further surface functionalisation with angiogenesis-targeting RGD peptides (Fig. 33E). The protocol rendered RCPs of 95\% with high RCS after incubation in human plasma. In addition, SPECT/CT imaging allowed the identification of tumours in a manner relevant to integrin overexpression (Fig. 33F). Pang et al. reported a straightforward, one-step protocol where ${ }^{199} \mathrm{Au}^{3+}$ is introduced in a low molar concentration during the growth step of the gold NPs (see Section 4.4.1). ${ }^{106}$ This synthesis - described as a seed-mediated synthesis due to the radionuclide doping during the growth step from a native Au seed - provided RCYs of $96 \%$. This was shown to be reproducible irrespective of differences in specific activities, depending on the initial
${ }^{199} \mathrm{Au}^{3+}$ concentration, and allowed quantitative RCP. This strategy seems as the most straightforward to incorporate $\mathrm{Au}$ radionuclides into the crystal lattice of gold NPs. Other reported strategies have utilised spark ignition to obtain gold NPs aerosols by a complex reaction set up, or used $\mathrm{H}^{198} \mathrm{AuCl}_{4}$ to prepare gold nanocages. However, these methods are highly complex and time-consuming. ${ }^{105,108}$

4.6.5 Quantum dots. Quantum dots (QDs) offer excellent semiconductor and optical properties with a broad variety of applications in biomedical imaging and sensing. ${ }^{699}$ Their optical properties allow the selection of NPs with a broad range of adsorption and emission wavelengths - with low light-bleaching profiles. $^{700}$ Different mediators have been applied for the radiolabelling of QDs with the majority of examples reporting nonchelator radiolabelling strategies (Table 18). Very recently, Tang et al. reported the radiolabelling of zinc sulfide $(\mathrm{ZnS})$ dots with ${ }^{64} \mathrm{Cu}$ or ${ }^{68} \mathrm{Ga}$ by a heterogeneous radioisotopic exchange protocol. ${ }^{701}$ The radiolabelling of $\mathrm{ZnS}$ dots with $\left[{ }^{64} \mathrm{Cu}\right] \mathrm{CuCl}_{2}$ or $\left[{ }^{68} \mathrm{Ga} \mathrm{GaCl}_{3}\right.$ at $37{ }^{\circ} \mathrm{C}$ for $15 \mathrm{~min}$, allowing $>95 \% \mathrm{RCY}$ for ${ }^{68} \mathrm{Ga}$ and $\sim 90 \%$ RCY for ${ }^{64} \mathrm{Cu}$. Furthermore, the radiolabelled QDs showed a high RCS up to $24 \mathrm{~h}$ in mouse blood.

The hot + cold precursors method has been applied for the radiolabelling of $\mathrm{QD}_{\mathrm{S}}$ with ${ }^{64} \mathrm{Cu},{ }^{109} \mathrm{Cd}$ or ${ }^{125 \mathrm{~m}} \mathrm{Te}$, which were 
A
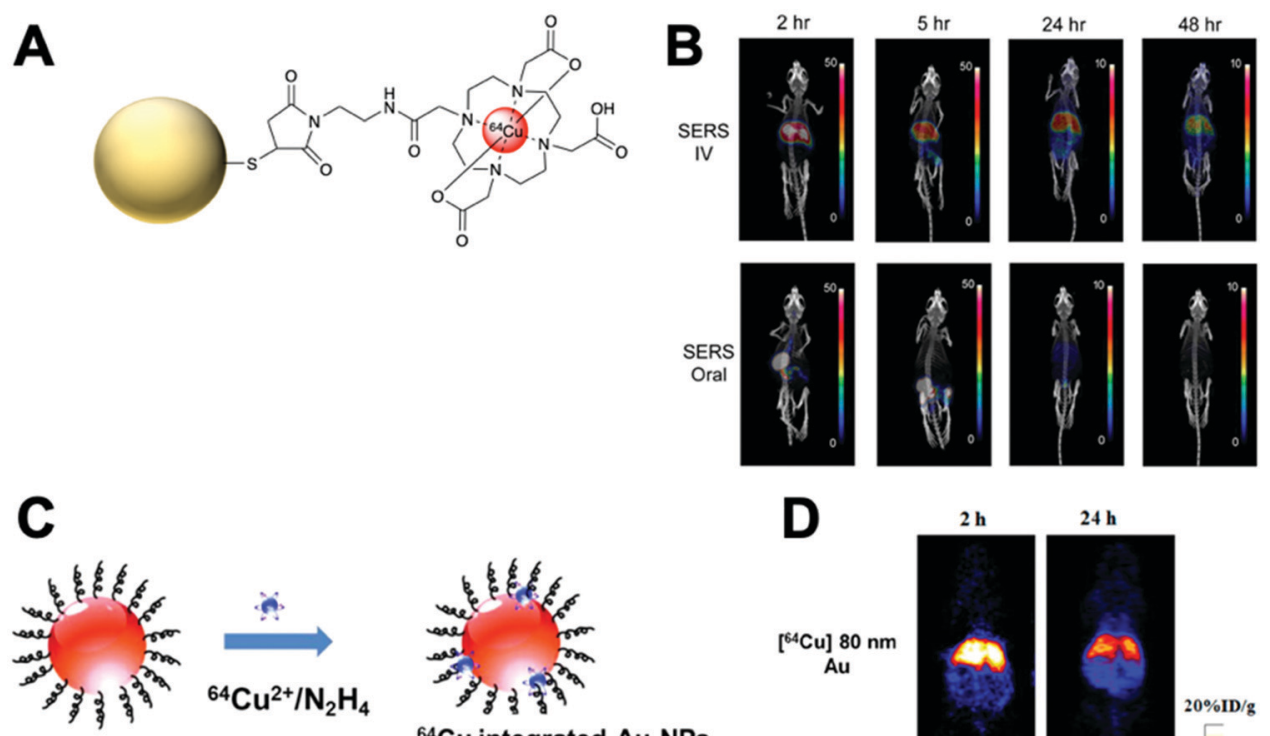

${ }^{64} \mathrm{Cu}$ integrated Au NPs
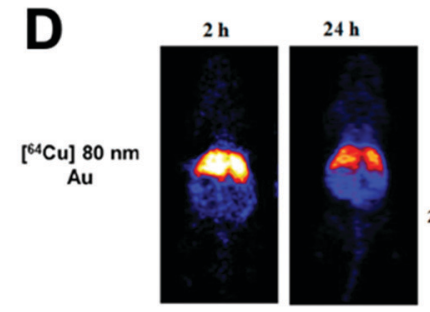

$20 \% \mathrm{ID} / \mathrm{g}$

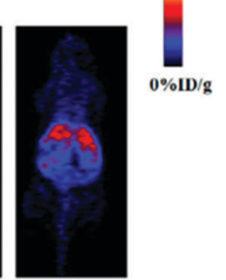

$\mathbf{F}$

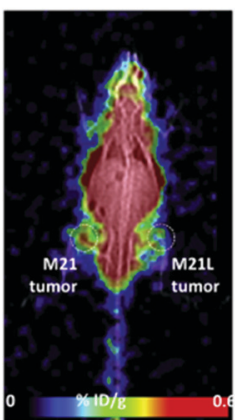

Fig. 33 (A) Schematic of Au-sulfur-maleimide-DOTA- ${ }^{64} \mathrm{Cu}$ NPs, (B) static microPET images at 2, 5, 24 and $48 \mathrm{~h}$ time points post IV or oral administration of radiolabelled SERS nanoparticles. Scale bar indicates \% injected dose per gram (\% ID per g) of tissue, (C) scheme of synthesis of chelator-free ${ }^{64} \mathrm{Cu}$-integrated Au NMs, (D) representative whole-body coronal PET images of mice at 2 and $24 \mathrm{~h}$ after intravenous injection of $130 \mu \mathrm{Ci}$ of $\left[{ }^{64} \mathrm{Cu}\right] 80 \mathrm{~nm}$ Au (upper) as well as free ${ }^{64} \mathrm{Cu}$ (lower), (E) design of the indium-111 labelled gold nanoparticles. Gold nanoparticles were synthesized with the particle core stably labelled with the $\gamma$-emitter indium-111 and the surface modified with linear and cyclic RGD ligands, (F) demonstrated higher uptake of RGD-modified indium-111 labeled gold nanoparticles in the M21 tumor (left) compared to the M21-L tumor (right). Adapted and reproduced with permission from ref. 659, 660 and 692 .

successfully incorporated into the core of CuInS/ZnS, CdSe/ CdZnS or CdTeSe/CdZnSe and CdTe QDs respectively. ${ }^{121-123}$ A key study by Guo et al. reported a one-pot synthesis of $\left[{ }^{64} \mathrm{Cu}\right] \mathrm{CIS} / \mathrm{ZnS}$ QDs from ${ }^{64} \mathrm{CuCl}_{2} / \mathrm{CuCl}_{2}, \mathrm{InCl}_{3}$ and $\mathrm{Na}_{2} \mathrm{~S}$ along with a $\mathrm{ZnCl}_{2}$ shell formation and then in situ capped with PEG and glutathione (GSH) (Fig. 34A). ${ }^{122}$ These QDs showed fluorescence in the near infrared (NIR) with a quantum yield (QY) of $25 \%$. From the radiochemical point of view, this synthesis provided a RCY of $c a .100 \%$ and quantitative RCS in mouse serum. In vivo biodistribution studies in tumour bearing mice showed higher accumulation of the PEGylated GSH- $\left[{ }^{64} \mathrm{Cu}\right] \mathrm{CIS} / \mathrm{ZnS}$ in the tumour than GSH- $\left[{ }^{64} \mathrm{Cu}\right] \mathrm{CIS} / \mathrm{ZnS}$ and free ${ }^{64} \mathrm{CuCl}_{2}$ due to the longer blood circulation time of the former that allows accumulation due to the EPR phenomenon. Despite the significant tumour uptake level differences, It is important to highlight the high tumour uptake of free ${ }^{64} \mathrm{CuCl}_{2}$, that as we discussed above (Section 3.5 and Table 5) shows significant tumour uptake and similar biodistribution compared to both $\left[{ }^{64} \mathrm{Cu}\right] \mathrm{CIS} / \mathrm{ZnS}$ formulations, and may complicate image analysis. Interestingly, non-PEGylated GSH- $\left[{ }^{64} \mathrm{Cu}\right] \mathrm{CIS} / \mathrm{ZnS}$ showed significant bladder uptake due to the renal clearance of NPs that are smaller that $5.5 \mathrm{~nm}$ (Fig. 34B). These results highlight this method as suitable 
Table 18 Table summarising the methods, radioisotopes and techniques employed to radiolabel quantum dots

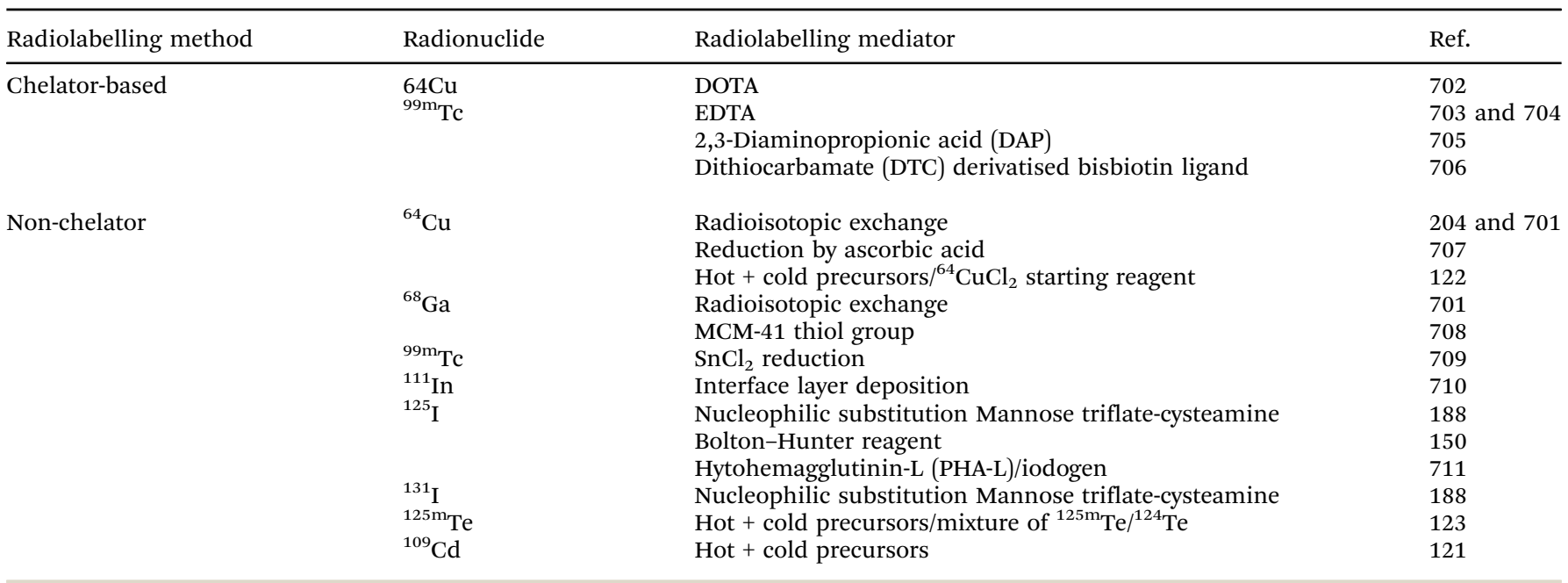

A

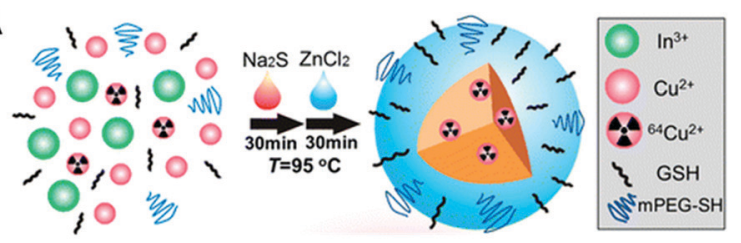

B

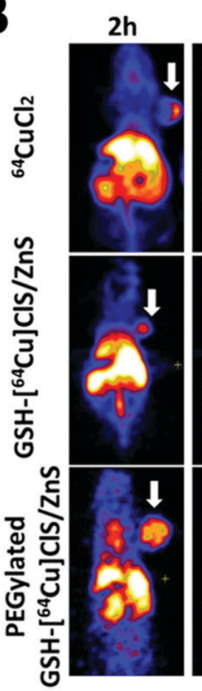

$6 \mathrm{~h}$
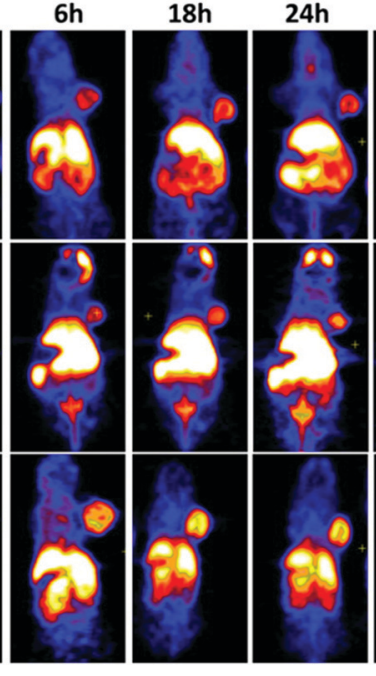

$48 \mathrm{~h}$

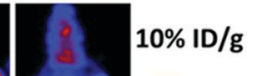

Fig. 34 (A) Illustration of the synthesis of intrinsically radioactive $\left[{ }^{64} \mathrm{Cu}\right] \mathrm{CIS} / \mathrm{ZnS}$ QDs, (B) representative whole-body coronal PET images of U87MG tumour-bearing mice at 2, 6, 18, 24, and $48 \mathrm{~h}$ after intravenous injection of $100 \mu \mathrm{L}(50 \mu \mathrm{g}, 300 \mu \mathrm{Ci})$ of ${ }^{64} \mathrm{CuCl}_{2}, \mathrm{GSH}-\left[{ }^{64} \mathrm{Cu}\right] \mathrm{CIS} / \mathrm{ZnS}$ and PEGylated GSH- $\left[{ }^{64} \mathrm{Cu}\right] \mathrm{CIS} / \mathrm{ZnS}$ RQDs. Arrow indicates location of the tumour. Adapted and reproduced with permission from ref. 122.

for the synthesis and radiolabelling of QDs in one step. Additionally, the protocol allowed the radiolabelling of QDs with ${ }^{111}$ In with the same favourable results, suggesting this protocol is a versatile and convenient strategy for QDs radiolabelling.

4.6.6 Up-converting nanoparticles. Up-converting nanoparticles (UCNP) have unique optical features with different applications for fluorescent microscopy, deep-tissue bioimaging, or as nanomedicines. ${ }^{712}$ These particles receive their name from their capacity to up-convert two or more photons of low energy in a single photon of high energy which results in their NIR excitation leading to $\mathrm{UV} /$ vis emission. ${ }^{713}$

Radiolabelling examples of these nanomaterials are quite recent with the first reports in 2011 (Table 19). As discussed in Section 4.4.4, the isotopic exchange between natural ${ }^{19} \mathrm{~F}$ and ${ }^{18} \mathrm{~F}$ is the main protocol for effective and robust radiolabelling of UCNPs. Other methods have also demonstrated successful incorporation of radionuclides. Rieffel et al. reported a chelator-based protocol using a porphyrin-phospholipid as NP coating. ${ }^{714}$ The coating provided a high affinity for ${ }^{64} \mathrm{Cu}$ by incubation at $37{ }^{\circ} \mathrm{C}$ and $\mathrm{pH}=5.5$ with RCYs greater than $80 \%$. Interestingly, the combination of only two components, the porphyrin-phospholipid and the UCNP core, rendered excellent capabilities for six different imaging modalities (FL/PA/ $\mathrm{PET} / \mathrm{CT} / \mathrm{CL} / \mathrm{UC}$ ). Alternatively, Yang et al. described a one-pot hydrothermal synthesis of $\mathrm{NaLuF}_{4}:{ }^{153} \mathrm{Sm}, \mathrm{Yb}, \mathrm{Tm}$ NPs. ${ }^{112}$ The use of $\left[{ }^{153} \mathrm{Sm}\right] \mathrm{SmCl}_{3}$ in the starting reagents provided a $100 \%$ RCY with $>99 \%$ RCS after incubation in FBS for $24 \mathrm{~h}$. This high stability was expected since the ${ }^{153} \mathrm{Sm}$ is incorporated in the crystal lattice of the particles.

Table 19 Table summarising the methods, radioisotopes and techniques employed to radiolabel up-converting nanoparticles

\begin{tabular}{llll}
\hline $\begin{array}{l}\text { Radiolabelling } \\
\text { method }\end{array}$ & Radionuclide & Radiolabelling mediator & Ref. \\
\hline Chelator-based & ${ }^{64} \mathrm{Cu}$ & NOTA & 715 \\
& & Porphyrin & 714 \\
& & Bisphosphonate & 716 \\
& ${ }^{68} \mathrm{Ga}$ & DOTA & 717 \\
& ${ }^{99 \mathrm{~m}} \mathrm{Tc}$ & Bisphosphonate & 716 \\
Non-chelator & ${ }^{18} \mathrm{~F}$ & Radioisotopic exchange & $198-201$ \\
& ${ }^{124} \mathrm{I}$ & Iodo-beads & 149 \\
& ${ }^{125} \mathrm{I}$ & Bolton-Hunter reagent & 135 \\
& ${ }^{153} \mathrm{Sm}$ & Hot + cold precursors & 112 \\
& ${ }^{90} \mathrm{Y}$ & Radioisotopic exchange & 205 \\
& ${ }^{12} \mathrm{Hot}+$ cold precursors & 120
\end{tabular}




\section{Applications of radiolabelled nanomaterials in imaging and therapy}

The radiolabelling of nanomaterials can be performed for a variety of different applications with their use encompassing pre-clinical validation all the way to imaging in a clinical setting. In this section, the main applications for using radiolabelled NPs will be outlined, with important and/or interesting examples briefly discussed.

\subsection{Assessment of new formulations}

One of the most widely used applications of radiolabelled nanomaterials is to assess the in vivo biodistribution of novel formulations. The sensitivity and quantitative nature of nuclear imaging easily allows elucidation of whole-body pharmacokinetics, biodistribution and target accumulation of different nanomaterials. A good example of this was reported by Tang et al. who created a library of lipoprotein-based NPs as candidates for atherosclerosis treatment. The leading candidates were radiolabelled with ${ }^{89} \mathrm{Zr}$, and their in vivo behaviour evaluated with PET imaging. ${ }^{366}$ This allowed comparison of their blood pharmacokinetics and uptake in organs of interest. As well as testing novel formulations, another key application is imaging the distribution of targeted NPs versus their nontargeted counterparts. For example, Yang et al. radiolabelled cRGD-functionalised SPIONs with ${ }^{64} \mathrm{Cu}$; demonstrating increased tumour accumulation in mice for the targeted NPs - compared with non-targeted SPIONs - which could be blocked via the administration of cRGD (Fig. 35A). ${ }^{718}$ On the other hand, Christensen et al. used PET imaging of folate-targeted liposomes labelled with ${ }^{64} \mathrm{Cu}$ to show there was lower uptake in high folate-receptor expressing tumours, compared with nontargeted liposomes. ${ }^{719}$ This suggested that the functionalised used for targeting may result in reduction of EPR-mediated uptake of the liposomes.

\subsection{Personalised nanomedicine}

A key application of radiolabelled nanomaterials is for the assessment of target accumulation in the patients undergoing treatment with nanomedicines. The EPR mechanism that often drives the accumulation of nanomedicines in target tissues is highly heterogenous in humans. ${ }^{723-725}$ However, by imaging nanomedicines non-invasively within patients, they can be grouped into potential responders and non-responders allowing treatment stratification - a concept known as personalised nanomedicine. ${ }^{726}$ A key clinical study was reported by Lee et al. who radiolabelled HER2-targeted liposomal doxorubicin (MM-302) with ${ }^{64} \mathrm{Cu}$ and performed PET imaging in patients with metastatic breast cancer. ${ }^{326}$ PET imaging showed heterogenous uptake of the liposomes in primary tumours and metastases; both from patient-to-patient and within lesions within the same patient. Despite this, a correlation was observed between tumour uptake of MM-302 and the patient's disease progression-free survival (Fig. 35B). As opposed to radiolabelling and imaging specific nanomedicines to assess their target accumulation, a potentially more robust method is to inject a 'companion diagnostic' which behaves similarly to or demonstrates EPR-mediated uptake. This concept has been previously demonstrated using the iron-oxide nanoparticle, Ferumoxytol. ${ }^{727}$ Both Perez-Medina et $a .^{271}$ and Lee et $a .^{327}$ developed radiolabelled nanoliposome platforms that could be injected both priorto or with the injection of nanomedicines - allowing prediction of therapeutic response in preclinical cancer models.

\subsection{Diagnostics}

Another application of radiolabelled nanomaterials is their use for diagnostics. The EPR-mediated uptake of NPs into tumours or sites of inflammation can clearly be taken advantage for diagnostic purposes. Mahakian et al. compared the diagnostic potential of long-circulating liposomes radiolabelled with ${ }^{64} \mathrm{Cu}$ with $\left[{ }^{18}\right.$ F]FDG in a head and neck cancer mouse model. The tumour accumulation and signal to background ratios of the labelled liposomes were superior to $\left[{ }^{18} \mathrm{~F}\right]$ FDG when imaging after $24 \mathrm{~h} .{ }^{260}$ However, in a clinical setting, the requirement for delayed imaging may be limiting and the longer half-life of the radioisotope may also result in a higher absorbed radiation dose. Additionally, radiolabelled NPs can be used as multimodal systems; to take advantage of the sensitivity of nuclear imaging in conjunction with more anatomical-focused modalities - such as MRI and CT. ${ }^{139}$ However, the use of NPs as multimodal diagnostics should arguably provide benefits over lower MW tracers, and when using single modalities. An example of this approach was reported by Savolainen et al. who radiolabelled Sienna+/Magtrace ${ }^{\circledR}$ (a macrophage-targeted clinically-approved SPIO) with ${ }^{68} \mathrm{Ga}$, as a tool for PET-MRI guided sentinel lymph node (SLN) biopsies in metastatic cancer. $^{720}$ In this approach, PET provides sensitive wholebody information of the location of the SLNs, while MRI provides information on their different macrophage levels and hence metastatic status (Fig. 35C).

\subsection{Cell tracking}

Cell tracking describes the use of medical imaging techniques to non-invasively image the biodistribution and trafficking of cells in a living organism. This information can be beneficial for: disease diagnosis, the imaging of biological mechanisms, and evaluating the efficacy of treatments. ${ }^{728}$ More recently, cell tracking methods have been used for the development and evaluation of cellular therapies - such as CAR T-cell immunotherapies. To allow cell tracking, cells often have to labelled with a contrast agent. For example, for cell tracking with nuclear imaging, radionuclides have to be incorporated into cells; often by their attachment to cellular membranes, or internalisation via the use of radio-ionophores (as in Section 4.3.1). Alternatively, nanomaterials can enter cells through a variety of different mechanisms including such as phagocytosis, endocytosis or micropinocytosis. ${ }^{729}$ Hence these mechanisms can be taken advantage of to enable the radiolabelling of cells, to facilitate in vivo cell tracking. A variety of different cell types have been radiolabelled with chitosan-based NPs, ${ }^{503,504}$ gold NPs, ${ }^{730,731}$ SPIONs, ${ }^{213,610,732}$ and mesoporous silica NPs. ${ }^{721,733}$ The efficiency of this cell labelling process is exemplified by 
A New formulations/targeting

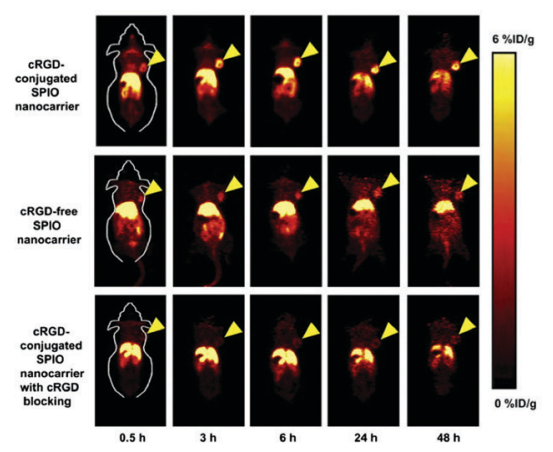

D

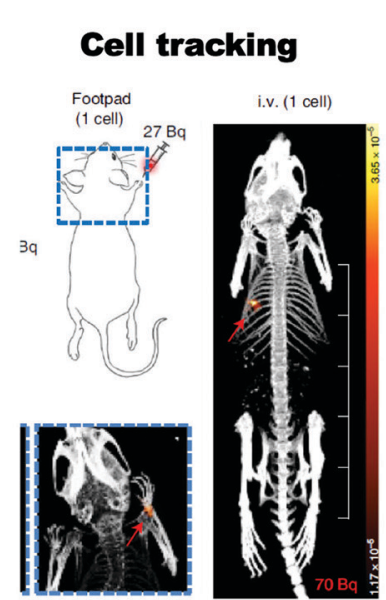

B
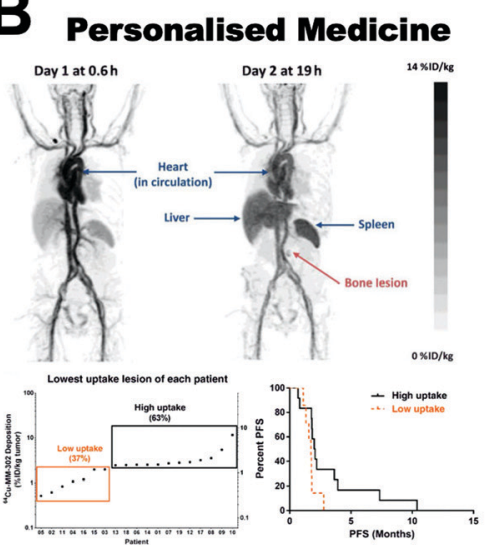

C

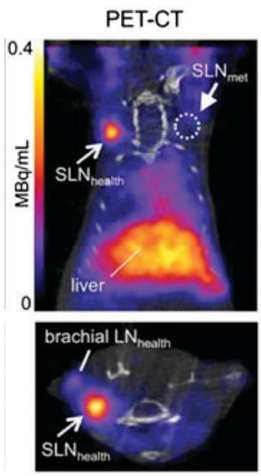

Diagnosis

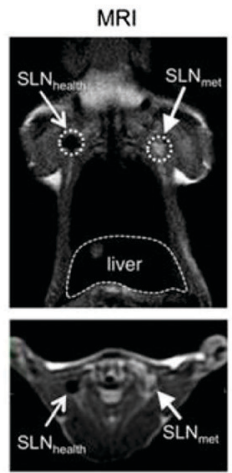

(a)
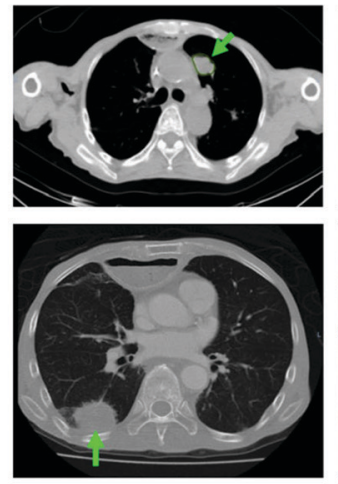

\section{Radiotherapy}

(b)
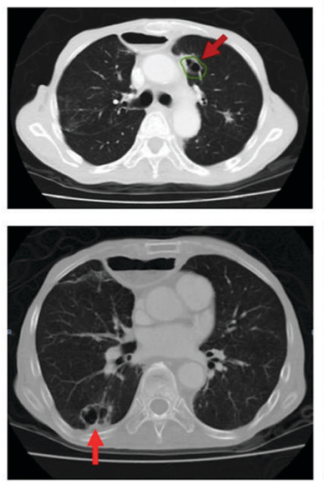

(c)

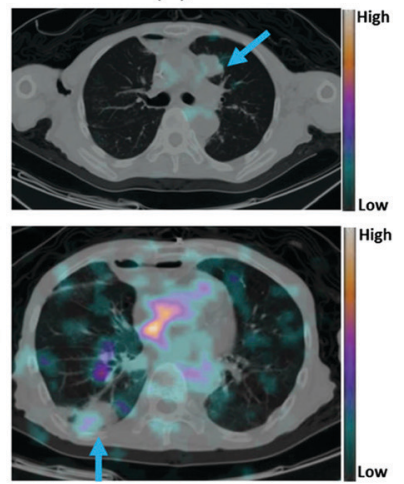

Fig. 35 Summary of the five main applications of radiolabelled nanoparticles focused on in this review. (A) The radiolabelling of nanoparticles can allow the testing of new formulations and the assessment of NP targeting. PET images of U87MG tumor-bearing mice at various time points post-injection of ${ }^{64} \mathrm{Cu}$-labeled SPIO nanocarriers (CRGD-conjugated, CRGD-free, and CRGD-conjugated with a blocking dose of cRGD). Figure adapted from Yang et al. ${ }^{718}$ (B) Radiolabelled nanoparticles can aid the clinical translation of nanoparticles and assess target engagement in patients: personalised nanomedicines. Maximum intensity projection PET images of 2 patients with HER2-positive breast cancer injected with ${ }^{64} \mathrm{Cu}$-labelled HER2-targeted liposomes (top). Patient lesion deposition of the lowest uptake lesion within each patient from days 2 or 3 are shown and aligned in ascending order (bottom left). Patient PFS of the high versus low uptake patients is shown in a Kaplan-Meier curve (bottom right). Figures adapted from Lee et al. ${ }^{326}$ (C) Radiolabelled nanoparticles can be used for the diagnosis of disease, in this case by exploiting multimodal PET-MR imaging and ${ }^{68} \mathrm{Ga}$-labelled SPIOs to locate and identify metastatic lymph nodes. Figure adapted from Savolainen et al. ${ }^{720}$ (D) Radiolabelled nanoparticles can be used as cell labelling agents, allowing the in vivo tracking of cells. A single MDA-MB-231 cells is imaged with PET in the paw (left) and lung (right) of a mouse. Figure from Jung et al. ${ }^{721}$ (E) Nanoparticles can be used for radiotherapy. CT images for patients with esophageal cancer and lung metastases tumors: 1 month before (left column)and 4 months after (middle column) administration of ${ }^{188} \mathrm{Re}$-liposome injection, the metastatic lesions (green arrows) either decrease in size or show signs of cavitation (red arrows). SPECT/CT images (right column) show a high uptake and efficient targeting of ${ }^{188} \mathrm{Re}$-liposome in the corresponding tumor lesion (blue arrow). Figure adapted from Wang et al. ${ }^{722}$

Jung et al. who used ${ }^{68} \mathrm{Ga}$-labelled MSNPs to radiolabel breast cancer cells with enough activity ( $c a .30 \mathrm{~Bq}$ per cell) to allow the in vivo tracking of a single cell using PET (Fig. 35D). ${ }^{721}$

\subsection{Radionuclide therapy}

Finally, nanomaterials can be labelled with therapeutic radionuclides (see Section 2.3) allowing them to be used for radionuclide therapy. As with their use as diagnostics, the accumulation of nanomaterials at tumour sites via the EPR effect or due to targeting can allow delivery of the radionuclide. However, the long-circulating properties of some nanomaterials may be considered a drawback in the context of radionuclide therapy due to the possible increase in radiation dose to the patient and non-target organs - such as the spleen. Wang et al. recently reported a phase 0 study of PEGylated liposomes radiolabelled with ${ }^{188} \mathrm{Re}$ (beta emitter) in patients with metastatic cancer. $^{722}$ A partial therapeutic effect was observed in some metastatic lesions, which also showed uptake of the radiolabelled liposomes (Fig. 35E). Despite this, dosimetry measurements showed the highest absorbed dose was in the spleen and liver. Whilst this work demonstrates clear progress in the use of nanomaterials for radiotherapy, the application of NPs for this purpose must show clear benefits - in terms of efficacy and vital organ dosimetry - over standard targeted radionuclide therapy methods (i.e. radiolabelled antibodies or small molecules). 
Besides radiotherapy applications, a novel therapeutic strategy based on Cerenkov luminescence (CL) is becoming a successful choice to induce a photodynamic therapy (PDT) response. This strategy, known as Cerenkov radiation-induced therapy (CRIT) leverage from the UV-blue light generated by the radionuclide decay that interacts with a photosensitive nanomaterial triggering the emission of long-wavelength photons that produce cytotoxic reactive oxygen species (ROS). ${ }^{734,735}$ Most of reported examples of CRIT applications are based on the combination of $\mathrm{TiO}_{2}$ nanoparticles as photosensitiser material with ${ }^{68} \mathrm{Ga},{ }^{64} \mathrm{Cu},{ }^{18} \mathrm{~F}$ or ${ }^{89} \mathrm{Zr}$ radionuclides to generate the CL. ${ }^{736-739}$ Apart of $\mathrm{TiO}_{2}$ nanoparticles, other materials such as iron oxide NPs and porphyrins have shown a remarkable PDT response in combination with ${ }^{89} \mathrm{Zr},{ }^{740,741}$ opening a very encouraging field within personalised nanomedicine due to the unique properties of the radiolabelled nanomaterials.

\section{Conclusions and perspectives}

In this review we have described and discussed the different radiochemical methods explored to date to radiolabel different nanomaterials of interest for medical applications, with applications in imaging and therapy. We believe there are several conclusions that we can draw from this work and should be taken into account when considering the best methodology for a specific NP-radiolabelling project. First, for each nanomaterial and formulation, each radiochemical approach available will have inherent advantages and disadvantages. Ultimately, the selection of methodology should be driven by its capabilities to provide the required information, but this is only achievable if we are aware of its pitfalls (known and potential). To facilitate this, we strongly believe it is important to incorporate as many control groups as required into any study involving the in vivo evaluation of radiolabelled NPs (e.g. radionuclide-only, radiochelate-only, radiolabelled component-only, etc.). Where relevant, it is also important to include ex vivo information to complement and support the in vivo results (e.g. histology methods). A particularly relevant aspect to take into account for in vivo studies is the fact that many radionuclides and radiochelates accumulate in, or are cleared by, the same organs as nanomaterials (e.g. liver, bone marrow, lungs, tumours), complicating image analysis in the absence of appropriate controls. Often, the selection of radiolabelling methodology will be limited by radionuclide/equipment availability, as this type of work requires special health and safety considerations and expensive facilities. However, we believe that the wide variety of methodologies and substantial knowledge already available for different radionuclides and nanomaterials, as described in this review, should allow ample choice for effective and informative radionuclide-based NP studies.

The same properties of nanomaterials that make them attractive for biomedical applications, result in some important disadvantages when compared to other imaging/therapy platforms. Importantly, their relatively large size and surface area makes them easy targets for phagocytic cells, limiting their ability to reach their intended target. This can be in part addressed by the use of stealth coatings, but ultimately their excretion is likely to involve the mononuclear phagocyte system (MPS), which is slow compared to the renal excretion often found for smaller molecules. In addition, this results in low tissue penetration, limiting their potential as imaging agents and drug carriers when the target is not easily accessible from the vasculature. However, some of these disadvantages can be exploited for specific purposes, for example by using NPs as myeloid cell-targeting agents, with wide-ranging applications from inflammation imaging to novel therapeutic approaches such as those based on trained-immunity. ${ }^{742-744}$

In terms of future applications, it is likely that radiolabelled NPs will be increasingly used as tools to inform the clinical translation of nanoparticles as therapeutics, and potentially as theranostic agents. As mentioned above, radiolabelling allows an accurate and sensitive method to study their biodistribution and pharmacokinetics in both animals and humans. Hence, we strongly believe that integrating these techniques early in the development of therapeutic nanomedicines will significantly enhance their clinical translation potential, by allowing the selection of the best candidates and de-risking the process. If a clear association between the biodistribution of a therapeutic NP and their biodistribution can be made, radiolabelled NPs have significant potential as predictors of efficacy. The capacity of NPs to carry several radionuclides per particle also makes them attractive as radionuclide therapy agents, but the slow excretion and retention of NPs remains a significant barrier to overcome that limits their potential. Finally, new developments in PET technology - specifically total-body $\mathrm{PET}^{44,45}$ - provide exciting opportunities for NP-based biomedical applications. These are mainly driven by the increased sensitivity (allowing NP imaging with short-lived radionuclides and lower radiation doses). We believe this will facilitate more PET imaging-based clinical evaluation of novel nanomedicines in the future, increasing the impact of this technology in the effective clinical translation of novel nanomedicines.

\section{Conflicts of interest}

Research in the RTMR group has received funding by AstraZeneca plc (PhD studentship), GlaxoSmithKline plc (PhD studentship), Theragnostics Ltd (PhD studentship) and Lipomedix Pharmaceutical Ltd (in kind contributions).

\section{Acknowledgements}

The authors are funded by EPSRC programme grants EP/ S032789/1 (Probing Multiscale Complex Multiphase Flows with Positrons for Engineering and Biomedical Applications) and EP/R045046/1 (Next Generation Molecular Imaging and Therapy with Radionuclides). We also acknowledge support from the Wellcome/EPSRC Centre for Medical Engineering [WT/203148/Z/16/Z] and the KCL and UCL Comprehensive Cancer Imaging Centre funded by CRUK and EPSRC in association with the MRC and DoH (England). Radioanalytical equipment was 
funded by a Wellcome Trust Multiuser Equipment Grant: A multiuser radioanalytical facility for molecular imaging and radionuclide therapy research $[212885 / \mathrm{Z} / 18 / \mathrm{Z}]$. The authors finally acknowledge support the National Institute for Health Research (NIHR) Biomedical Research Centre based at Guy's and St Thomas' NHS Foundation Trust and KCL [grant number IS-BRC-121520006]. The views expressed are those of the authors and not necessarily those of the NHS, the NIHR or the Department of Health.

\section{References}

1 B. Pelaz, C. Alexiou, R. A. Alvarez-Puebla, F. Alves, A. M. Andrews, S. Ashraf, L. P. Balogh, L. Ballerini, A. Bestetti, C. Brendel, S. Bosi, M. Carril, W. C. W. Chan, C. Chen, X. Chen, X. Chen, Z. Cheng, D. Cui, J. Du, C. Dullin, A. Escudero, N. Feliu, M. Gao, M. George, Y. Gogotsi, A. Grünweller, Z. Gu, N. J. Halas, N. Hampp, R. K. Hartmann, M. C. Hersam, P. Hunziker, J. Jian, X. Jiang, P. Jungebluth, P. Kadhiresan, K. Kataoka, A. Khademhosseini, J. Kopeček, N. A. Kotov, H. F. Krug, D. S. Lee, C.-M. Lehr, K. W. Leong, X.-J. Liang, M. Ling Lim, L. M. Liz-Marzán, X. Ma, P. Macchiarini, H. Meng, H. Möhwald, P. Mulvaney, A. E. Nel, S. Nie, P. Nordlander, T. Okano, J. Oliveira, T. H. Park, R. M. Penner, M. Prato, V. Puntes, V. M. Rotello, A. Samarakoon, R. E. Schaak, Y. Shen, S. Sjöqvist, A. G. Skirtach, M. G. Soliman, M. M. Stevens, H.-W. Sung, B. Z. Tang, R. Tietze, B. N. Udugama, J. S. VanEpps, T. Weil, P. S. Weiss, I. Willner, Y. Wu, L. Yang, Z. Yue, Q. Zhang, Q. Zhang, X.-E. Zhang, Y. Zhao, X. Zhou and W. J. Parak, ACS Nano, 2017, 11, 2313-2381.

2 A. C. Anselmo and S. Mitragotri, Bioeng. Transl. Med., 2016, 1, 10-29.

3 S. T. Duggan and G. M. Keating, Drugs, 2011, 71, 2531-2558.

4 A. C. Anselmo and S. Mitragotri, Bioeng. Transl. Med., 2019, 4, e10143.

5 F. C. Passero, D. Grapsa, K. N. Syrigos and M. W. Saif, Expert Rev. Anticancer Ther., 2016, 16, 697-703.

6 R. A. Schwendener, Ther. Adv. Vaccines, 2014, 2, 159-182.

7 H.-I. Chang and M.-K. Yeh, Int. J. Nanomed., 2012, 7, 49-60.

8 D. Girelli, S. Ugolini, F. Busti, G. Marchi and A. Castagna, Int. J. Hematol., 2018, 107, 16-30.

9 Y. Min, J. M. Caster, M. J. Eblan and A. Z. Wang, Chem. Rev., 2015, 115, 11147-11190.

10 S. Kunjachan, J. Ehling, G. Storm, F. Kiessling and T. Lammers, Chem. Rev., 2015, 115, 10907-10937.

11 R. van der Meel, E. Sulheim, Y. Shi, F. Kiessling, W. J. M. Mulder and T. Lammers, Nat. Nanotechnol., 2019, 14, 1007-1017.

12 R. Bar-Shalom, A. Y. Valdivia and M. D. Blaufox, Semin. Nucl. Med., 2000, 30, 150-185.

13 E. C. Pratt, T. M. Shaffer and J. Grimm, Wiley Interdiscip. Rev.: Nanomed. Nanobiotechnol., 2016, 8, 872-890.
14 E.-K. Lim, T. Kim, S. Paik, S. Haam, Y.-M. Huh and K. Lee, Chem. Rev., 2015, 115, 327-394.

15 S. Mura and P. Couvreur, Adv. Drug Delivery Rev., 2012, 64, 1394-1416.

16 H. Chen, W. Zhang, G. Zhu, J. Xie and X. Chen, Nat. Rev. Mater., 2017, 2, 17024.

17 S. D. Jo, S. H. Ku, Y.-Y. Won, S. H. Kim and I. C. Kwon, Theranostics, 2016, 6, 1362-1377.

18 M. Hamoudeh, M. A. Kamleh, R. Diab and H. Fessi, Adv. Drug Delivery Rev., 2008, 60, 1329-1346.

19 M. L. James and S. S. Gambhir, Physiol. Rev., 2012, 92, 897-965.

20 C. Pérez-Medina, A. J. P. Teunissen, E. Kluza, W. J. M. Mulder and R. van der Meel, Adv. Drug Delivery Rev., 2020, 154-155, 123-141.

21 K. Heinzmann, L. M. Carter, J. S. Lewis and E. O. Aboagye, Nat. Biomed. Eng., 2017, 1, 697-713.

22 J. Weber, P. C. Beard and S. E. Bohndiek, Nat. Methods, 2016, 13, 639-650.

23 X. Han, K. Xu, O. Taratula and K. Farsad, Nanoscale, 2019, 11, 799-819.

24 A. K. Gupta and M. Gupta, Biomaterials, 2005, 26, 3995-4021.

25 I. Fernández-Barahona, M. Muñoz-Hernando, J. RuizCabello, F. Herranz and J. Pellico, Inorganics, 2020, 8.

26 J. Y. Park, M. J. Baek, E. S. Choi, S. Woo, J. H. Kim, T. J. Kim, J. C. Jung, K. S. Chae, Y. Chang and G. H. Lee, ACS Nano, 2009, 3, 3663-3669.

27 J. Pellico, J. Ruiz-Cabello, I. Fernández-Barahona, L. Gutiérrez, A. V. Lechuga-Vieco, J. A. Enríquez, M. P. Morales and F. Herranz, Langmuir, 2017, 33, 10239-10247.

28 M. L. Senders, A. E. Meerwaldt, M. M. T. van Leent, B. L. Sanchez-Gaytan, J. C. van de Voort, Y. C. Toner, A. Maier, E. D. Klein, N. A. T. Sullivan, A. M. Sofias, H. Groenen, C. Faries, R. S. Oosterwijk, E. M. van Leeuwen, F. Fay, E. Chepurko, T. Reiner, R. Duivenvoorden, L. Zangi, R. M. Dijkhuizen, S. Hak, F. K. Swirski, M. Nahrendorf, C. Pérez-Medina, A. J. P. Teunissen, Z. A. Fayad, C. Calcagno, G. J. Strijkers and W. J. M. Mulder, Nat. Nanotechnol., 2020, 1-8.

29 P. Chhour, P. C. Naha, S. M. O'Neill, H. I. Litt, M. P. Reilly, V. A. Ferrari and D. P. Cormode, Biomaterials, 2016, 87, 93-103.

30 S. A. Peyman, J. R. McLaughlan, R. H. Abou-Saleh, G. Marston, B. R. G. Johnson, S. Freear, P. L. Coletta, A. F. Markham and S. D. Evans, Lab Chip, 2016, 16, 679-687.

31 J. Gao, K. Chen, R. Luong, D. M. Bouley, H. Mao, T. Qiao, S. S. Gambhir and Z. Cheng, Nano Lett., 2012, 12, 281-286.

32 A. de la Zerda, Z. Liu, S. Bodapati, R. Teed, S. Vaithilingam, B. T. Khuri-Yakub, X. Chen, H. Dai and S. S. Gambhir, Nano Lett., 2010, 10, 2168-2172.

33 S. Imlimthan, S. Otaru, O. Keinänen, A. Correia, K. Lintinen, H. A. Santos, A. J. Airaksinen, M. A. Kostiainen and M. Sarparanta, Biomacromolecules, 2019, 20, 674-683.

34 F. Chen, K. Ma, L. Zhang, B. Madajewski, P. Zanzonico, S. Sequeira, M. Gonen, U. Wiesner and M. S. Bradbury, Chem. Mater., 2017, 29, 8269-8281. 
35 J. Kim, P. Chhour, J. Hsu, H. I. Litt, V. A. Ferrari, R. Popovtzer and D. P. Cormode, Bioconjugate Chem., 2017, 28, 1581-1597.

36 M. Shilo, T. Reuveni, M. Motiei and R. Popovtzer, Nanomedicine, 2012, 7, 257-269.

37 D. Xi, S. Dong, X. Meng, Q. Lu, L. Meng and J. Ye, RSC Adv., 2012, 2, 12515-12524.

38 H.-D. Liang and M. J. K. Blomley, Br. J. Radiol., 2003, 76, S140-S150.

39 K. Christensen-Jeffries, O. Couture, P. A. Dayton, Y. C. Eldar, K. Hynynen, F. Kiessling, M. O’Reilly, G. F. Pinton, G. Schmitz, M. X. Tang, M. Tanter and R. J. G. van Sloun, Ultrasound Med. Biol., 2020, 46, 865-891.

40 V. Ntziachristos and D. Razansky, Chem. Rev., 2010, 110, 2783-2794.

41 L. Zhou, H. Zhou and C. Wu, Wiley Interdiscip. Rev.: Nanomed. Nanobiotechnol., 2018, 10, e1510.

42 M. M. Khalil, J. L. Tremoleda, T. B. Bayomy and W. Gsell, Int. J. Mol. Imaging, 2011, 2011, 1-15.

43 T. Fukuchi, T. Okauchi, M. Shigeta, S. Yamamoto, Y. Watanabe and S. Enomoto, Med. Phys., 2017, 44, 2257-2266.

44 S. R. Cherry, T. Jones, J. S. Karp, J. Qi, W. W. Moses and R. D. Badawi, J. Nucl. Med., 2018, 59, 3-12.

45 R. D. Badawi, H. Shi, P. Hu, S. Chen, T. Xu, P. M. Price, Y. Ding, B. A. Spencer, L. Nardo, W. Liu, J. Bao, T. Jones, H. Li and S. R. Cherry, J. Nucl. Med., 2019, 60, 299-303.

46 M. Silindir-Gunay, M. Karpuz and A. Y. Ozer, Cancer Biother. Radiopharm., 2020, 35(6), 446-458, DOI: 10.1089/ cbr.2019.3213.

47 J. Jeon, Int. J. Mol. Sci., 2019, 20, 2323.

48 S. Poty, L. C. Francesconi, M. R. McDevitt, M. J. Morris and J. S. Lewis, J. Nucl. Med., 2018, 59, 878-884.

49 S. Aghevlian, A. J. Boyle and R. M. Reilly, Adv. Drug Delivery Rev., 2017, 109, 102-118.

50 K. E. Baidoo, K. Yong and M. W. Brechbiel, Clin. Cancer Res., 2013, 19, 530-537.

51 A. Ku, V. J. Facca, Z. Cai and R. M. Reilly, EJNMMI Radiopharm. Chem., 2019, 4, 27.

52 K. P. Willowson, Eur. J. Phys., 2019, 40, 043001.

53 K. Peach, P. Wilson and B. Jones, Br. J. Radiol., 2011, 84(1), $\mathrm{S} 4-\mathrm{S} 10$.

54 A. Dash and R. Chakravarty, Am. J. Nucl. Med. Mol. Imaging, 2019, 9, 30-66.

55 J. Magill and J. Galy, Radioactivity Radionuclides Radiation, Springer-Verlag, Berlin/Heidelberg, 2005.

56 K. F. Eckerman and A. Endo, MIRD: radionuclide data and decay schemes, Society of Nuclear Med, 2008.

57 A. Boschi, L. Uccelli and P. Martini, Appl. Sci., 2019, 9, 2526.

58 S. L. Pimlott and A. Sutherland, Chem. Soc. Rev., 2011, 40, 149-162.

59 G. Vaidyanathan and M. R. Zalutsky, Radiopharmaceutical Chemistry, Springer International Publishing, Cham, 2019, pp. 391-408.

60 J. Ródenas and E. Jabaloyas, J. Radioanal. Nucl. Chem., 2019, 322, 1691-1695.
61 Fluorine and Health, ed. A. Tressaud and G. Haufe, Elsevier, 2008.

62 M. M. Alauddin, Am. J. Nucl. Med. Mol. Imaging, 2012, 2, 55-76.

63 I. Verel, G. W. M. Visser, R. Boellaard, M. Stigter-van Walsum, G. B. Snow and G. A. M. S. van Dongen, J. Nucl. Med., 2003, 44, 1271-1281.

64 J. Zhang-Yin, C. Provost, G. Cancel-Tassin, T. Rusu, M. Penent, C. Radulescu, E. Comperat, O. Cussenot, F. Montravers, R. Renard-Penna, J.-N. Talbot and A. Prignon, Nucl. Med. Biol., 2020, 84-85, 88-95.

65 C.-H. Yeong, M. Cheng and K.-H. Ng, J. Zhejiang Univ., Sci., $B, 2014,15,845-863$.

66 C. Parker, S. Nilsson, D. Heinrich, S. I. Helle, J. M. O'Sullivan, S. D. Fosså, A. Chodacki, P. Wiechno, J. Logue, M. Seke, A. Widmark, D. C. Johannessen, P. Hoskin, D. Bottomley, N. D. James, A. Solberg, I. Syndikus, J. Kliment, S. Wedel, S. Boehmer, M. Dall'Oglio, L. Franzén, R. Coleman, N. J. Vogelzang, C. G. O'Bryan-Tear, K. Staudacher, J. Garcia-Vargas, M. Shan, Ø. S. Bruland and O. Sartor, N. Engl. J. Med., 2013, 369, 213-223.

67 C. Kratochwil, F. Bruchertseifer, F. L. Giesel, M. Weis, F. A. Verburg, F. Mottaghy, K. Kopka, C. Apostolidis, U. Haberkorn and A. Morgenstern, J. Nucl. Med., 2016, 57, 1941-1944.

68 T. Langbein, W. A. Weber and M. Eiber, J. Nucl. Med., 2019, 60, 13S-19S.

69 C. Kratochwil, U. Haberkorn and F. L. Giesel, Semin. Nucl. Med., 2020, 50, 133-140.

70 T. E. Witzig, L. I. Gordon, F. Cabanillas, M. S. Czuczman, C. Emmanouilides, R. Joyce, B. L. Pohlman, N. L. Bartlett, G. A. Wiseman, N. Padre, A. J. Grillo-López, P. Multani and C. A. White, J. Clin. Oncol., 2002, 20, 2453-2463.

71 H. Herzog, F. Rösch, G. Stöcklin, C. Lueders, S. M. Qaim and L. E. Feinendegen, J. Nucl. Med., 1993, 34, 2222-2226.

72 S. M. Qaim, B. Scholten and B. Neumaier, J. Radioanal. Nucl. Chem., 2018, 318, 1493-1509.

73 F. Man, P. J. Gawne and R. T. M. de Rosales, Adv. Drug Delivery Rev., 2019, 143, 134-160.

74 M. Quastel, A. Richter and J. Levy, Br. J. Cancer, 1990, 62, 885-890.

75 P. R. Franken, J. Guglielmi, C. Vanhove, M. Koulibaly, M. Defrise, J. Darcourt and T. Pourcher, Thyroid, 2010, 20, 519-526.

76 B. N. Bottenus, P. Kan, T. Jenkins, B. Ballard, T. L. Rold, C. Barnes, C. Cutler, T. J. Hoffman, M. A. Green and S. S. Jurisson, Nucl. Med. Biol., 2010, 37, 41-49.

77 J. Czernin, N. Satyamurthy and C. Schiepers, J. Nucl. Med., 2010, 51, 1826-1829.

78 R. G. Sephton, G. S. Hodgson, S. De Abrew and A. W. Harris, J. Nucl. Med., 1978, 19, 930-935.

79 H. M. Chilton, R. L. Witcofski, N. E. Watson and C. M. Heise, J. Nucl. Med., 1981, 22, 1064-1068.

80 J. Spetz, N. Rudqvist and E. Forssell-Aronsson, Cancer Biother. Radiopharm., 2013, 28, 657-664.

81 R. Chakravarty, S. Chakraborty and A. Dash, Mol. Pharmaceutics, 2016, 13, 3601-3612. 
82 A. Piccardo, F. Paparo, M. Puntoni, S. Righi, G. Bottoni, L. Bacigalupo, S. Zanardi, A. DeCensi, G. Ferrarazzo, M. Gambaro, F. G. Ruggieri, F. Campodonico, L. Tomasello, L. Timossi, S. Sola, E. Lopci and M. Cabria, J. Nucl. Med., 2018, 59, 444-451.

83 D. S. Abou, T. Ku and P. M. Smith-Jones, Nucl. Med. Biol., 2011, 38, 675-681.

84 S. A. Graves, R. Hernandez, J. Fonslet, C. G. England, H. F. Valdovinos, P. A. Ellison, T. E. Barnhart, D. R. Elema, C. P. Theuer, W. Cai, R. J. Nickles and G. W. Severin, Bioconjugate Chem., 2015, 26, 2118-2124.

85 A. Vakili, A. R. Jalilian, K. Yavari, S. Shirvani-Arani, A. Khanchi, A. Bahrami-Samani, B. Salimi and A. KhorramiMoghadam, J. Radioanal. Nucl. Chem., 2013, 296, 1287-1294.

86 W. A. Breeman, K. van der Wansem, B. F. Bernard, A. van Gameren, J. L. Erion, T. J. Visser, E. P. Krenning and M. de Jong, Eur. J. Nucl. Med. Mol. Imaging, 2003, 30, 312-315.

87 E. Dadachova, B. Bouzahzah, L. Zuckier and R. Pestell, Nucl. Med. Biol., 2002, 29, 13-18.

88 S. Hanadate, K. Washiyama, M. Yoshimoto, H. Matsumoto, A. Tsuji, T. Higashi and Y. Yoshii, J. Nucl. Med., 2017, 58, 1030.

89 P. Sukthankar, L. A. Avila, S. K. Whitaker, T. Iwamoto, A. Morgenstern, C. Apostolidis, K. Liu, R. P. Hanzlik, E. Dadachova and J. M. Tomich, Biochim. Biophys. Acta, Biomembr., 2014, 1838, 2296-2305.

90 H. H. Coenen, A. D. Gee, M. Adam, G. Antoni, C. S. Cutler, Y. Fujibayashi, J. M. Jeong, R. H. Mach, T. L. Mindt, V. W. Pike and A. D. Windhorst, Ann. Nucl. Med., 2018, 32, 236-238.

91 C. C. Wagner and O. Langer, Adv. Drug Delivery Rev., 2011, 63, 539-546.

92 J. J. M. de Goeij and M. L. Bonardi, J. Radioanal. Nucl. Chem., 2005, 263, 13-18.

93 S. E. Lapi and M. J. Welch, Nucl. Med. Biol., 2013, 40, 314-320.

94 E. W. Price and C. Orvig, Chem. Soc. Rev., 2014, 43, 260-290.

95 T. I. Kostelnik and C. Orvig, Chem. Rev., 2019, 119, 902-956.

96 T. J. Wadas, E. H. Wong, G. R. Weisman and C. J. Anderson, Chem. Rev., 2010, 110, 2858-2902.

97 G. T. Hermanson, Bioconjugate Techniques, Elsevier Inc., 3rd edn, 2013.

98 G. T. Hermanson, Bioconjugate Techniques, Elsevier, 2008, pp. 169-212.

99 J.-P. Meyer, P. Adumeau, J. S. Lewis and B. M. Zeglis, Bioconjugate Chem., 2016, 27, 2791-2807.

100 C. F. Ramogida and C. Orvig, Chem. Commun., 2013, 49, 4720.

101 B. M. Zeglis and J. S. Lewis, Dalton Trans., 2011, 40, 6168.

102 A. Steinbrueck, A. C. Sedgwick, J. T. Brewster, K.-C. Yan, Y. Shang, D. M. Knoll, G. I. Vargas-Zúñiga, X.-P. He, H. Tian and J. L. Sessler, Chem. Soc. Rev., 2020, 49, 3726-3747, DOI: 10.1039/C9CS00373H.

103 S. Goel, F. Chen, E. B. Ehlerding and W. Cai, Small, 2014, 10, 3825-3830.
104 J. Lamb and J. P. Holland, J. Nucl. Med., 2018, 59, 382-389. 105 W. G. Kreyling, W. Möller, U. Holzwarth, S. Hirn, A. Wenk, C. Schleh, M. Schäffler, N. Haberl, N. Gibson and J. C. Schittny, ACS Nano, 2018, 12, 7771-7790.

106 Y. Zhao, B. Pang, H. Luehmann, L. Detering, X. Yang, D. Sultan, S. Harpstrite, V. Sharma, C. S. Cutler, Y. Xia and Y. Liu, Adv. Healthcare Mater., 2016, 5, 928-935.

107 N. Chanda, V. Kattumuri, R. Shukla, A. Zambre, K. Katti, A. Upendran, R. R. Kulkarni, P. Kan, G. M. Fent, S. W. Casteel, C. J. Smith, E. Boote, J. D. Robertson, C. Cutler, J. R. Lever, K. V. Katti and R. Kannan, Proc. Natl. Acad. Sci. U. S. A., 2010, 107, 8760-8765.

108 Y. Wang, Y. Liu, H. Luehmann, X. Xia, D. Wan, C. Cutler and Y. Xia, Nano Lett., 2013, 13, 581-585.

109 R. Weissleder, D. Stark, B. Engelstad, B. Bacon, C. Compton, D. White, P. Jacobs and J. Lewis, Am. J. Roentgenol., 1989, 152, 167-173.

110 C. Chouly, D. Pouliquen, I. Lucet, J. J. Jeune and P. Jallet, J. Microencapsulation, 1996, 13, 245-255.

111 D. Pouliquen, R. Perdrisot, A. Ermias, S. Akoka, P. Jallet and J. Le Jeune, Magn. Reson. Imaging, 1989, 7, 619-627.

112 Y. Yang, Y. Sun, T. Cao, J. Peng, Y. Liu, Y. Wu, W. Feng, Y. Zhang and F. Li, Biomaterials, 2013, 34, 774-783.

113 Y. Zhao, B. Pang, H. Luehmann, L. Detering, X. Yang, D. Sultan, S. Harpstrite, V. Sharma, C. S. Cutler, Y. Xia and Y. Liu, Adv. Healthcare Mater., 2016, 5, 928-935.

114 J. Pellico, J. Ruiz-Cabello, M. Saiz-Alía, G. del Rosario, S. Caja, M. Montoya, L. Fernández de Manuel, M. P. Morales, L. Gutiérrez, B. Galiana, J. A. Enríquez and F. Herranz, Contrast Media Mol. Imaging, 2016, 11, 203-210.

115 J. Pellico, J. Ruiz-Cabello, M. Saiz-Alía, G. del Rosario, S. Caja, M. Montoya, L. Fernández de Manuel, M. P. Morales, L. Gutiérrez, B. Galiana, J. A. Enríquez and F. Herranz, Contrast Media Mol. Imaging, 2016, 11, 203-210.

116 H. Zolata, H. Afarideh and F. A. Davani, Cancer Biother. Radiopharm., 2016, 31, 324-329.

117 R. M. Wong, D. A. Gilbert, K. Liu and A. Y. Louie, ACS Nano, 2012, 6, 3461-3467.

118 E. Cędrowska, M. Pruszyński, W. Gawęda, M. Żuk, P. Krysiński, F. Bruchertseifer, A. Morgenstern, M.-A. Karageorgou, P. Bouziotis and A. Bilewicz, Molecules, 2020, 25, 1025.

119 J. Zeng, B. Jia, R. Qiao, C. Wang, L. Jing, F. Wang and M. Gao, Chem. Commun., 2014, 50, 2170.

120 S. Najmr, T. Lu, A. W. Keller, M. Zhang, J. D. Lee, M. Makvandi, D. A. Pryma, C. R. Kagan and C. B. Murray, Nano Futures, 2018, 2, 025002.

121 M. Sun, D. Hoffman, G. Sundaresan, L. Yang, N. Lamichhane and J. Zweit, Am. J. Nucl. Med. Mol. Imaging, 2012, 2, 122-135.

122 W. Guo, X. Sun, O. Jacobson, X. Yan, K. Min, A. Srivatsan, G. Niu, D. O. Kiesewetter, J. Chang and X. Chen, ACS Nano, 2015, 9, 488-495.

123 S. J. Kennel, J. D. Woodward, A. J. Rondinone, J. Wall, Y. Huang and S. Mirzadeh, Nucl. Med. Biol., 2008, 35, 501-514. 
124 L. Yang, G. Sundaresan, M. Sun, P. Jose, D. Hoffman, P. R. McDonagh, N. Lamichhane, C. S. Cutler, J. M. Perez and J. Zweit, J. Mater. Chem. B, 2013, 1, 1421.

125 T. M. Sakr, O. M. Khowessah, M. A. Motaleb, A. Abd ElBary, M. T. El-Kolaly and M. M. Swidan, Eur. J. Pharm. Sci., 2018, 122, 239-245.

126 X. Sun, W. Cai and X. Chen, Acc. Chem. Res., 2015, 48, 286-294.

127 C. Pérez-Campaña, V. Gómez-Vallejo, A. Martin, E. San Sebastián, S. E. Moya, T. Reese, R. F. Ziolo and J. Llop, Analyst, 2012, 137, 4902.

128 C. Pérez-Campaña, V. Gómez-Vallejo, M. Puigivila, A. Martín, T. Calvo-Fernández, S. E. Moya, R. F. Ziolo, T. Reese and J. Llop, ACS Nano, 2013, 7, 3498-3505.

129 I. Munaweera, Y. Shi, B. Koneru, R. Saez, A. Aliev, A. J. Di Pasqua and K. J. Balkus, Mol. Pharmaceutics, 2015, 12, 3588-3596.

130 W. M. da Silva, R. H. de Andrade Alves e Silva, M. F. Cipreste, G. F. Andrade, P. L. Gastelois, W. A. de Almeida Macedo and E. M. B. de Sousa, Appl. Radiat. Isot., 2020, 157, 109032.

131 T. Tsoncheva and E. Sarkadi-Priboczki, Microporous Mesoporous Mater., 2012, 148, 1-7.

132 R. Sharma, Y. Xu, S. W. Kim, M. J. Schueller, D. Alexoff, S. D. Smith, W. Wang and D. Schlyer, Nanoscale, 2013, $5,7476$.

133 M. Eisenhut and W. Mier, Handbook of Nuclear Chemistry, Springer US, Boston, MA, 2011, pp. 2121-2141.

134 H. H. Coenen, J. Mertens and B. Mazière, Radioionidation Reactions for Radio Pharmaceuticals, Springer Netherlands, Dordrecht, 2006, pp. 29-72.

135 U. Kostiv, V. Lobaz, J. Kučka, P. Švec, O. Sedláček, M. Hrubý, O. Janoušková, P. Francová, V. Kolářová, L. Šefc and D. Horák, Nanoscale, 2017, 9, 16680-16688.

136 K. C. L. Black, W. J. Akers, G. Sudlow, B. Xu, R. Laforest and S. Achilefu, Nanoscale, 2015, 7, 440-444.

137 K. C. L. Black, A. Ibricevic, S. P. Gunsten, J. A. Flores, T. P. Gustafson, J. E. Raymond, S. Samarajeewa, R. Shrestha, S. E. Felder, T. Cai, Y. Shen, A.-K. Löbs, N. Zhegalova, D. H. Sultan, M. Berezin, K. L. Wooley, Y. Liu and S. L. Brody, Biomaterials, 2016, 98, 53-63.

138 C. Pérez-Medina, N. Patel, M. Robson, M. F. Lythgoe and E. Årstad, Bioorg. Med. Chem. Lett., 2013, 23, 5170-5173.

139 J. Ge, Q. Zhang, J. Zeng, Z. Gu and M. Gao, Biomaterials, 2020, 228, 119553.

140 H. Sun, B. Zhang, X. Jiang, H. Liu, S. Deng, Z. Li and H. Shi, Nanomedicine, 2019, 14, 5-17.

141 S. Deng, W. Zhang, B. Zhang, R. Hong, Q. Chen, J. Dong, Y. Chen, Z. Chen and Y. Wu, J. Nanopart. Res., 2015, 17, 19.

142 A. L. B. de Barros, A.-M. Chacko, J. L. Mikitsh, A. Al Zaki, A. Salavati, B. Saboury, A. Tsourkas and A. Alavi, Mol. Imaging Biol., 2014, 16, 330-339, DOI: 10.1007/s11307013-0709-9.

143 R. Kumar, I. Roy, T. Y. Ohulchanskky, L. A. Vathy, E. J. Bergey, M. Sajjad and P. N. Prasad, ACS Nano, 2010, 4, 699-708.
144 G. Xie, J. Sun, G. Zhong, L. Shi and D. Zhang, Arch. Toxicol., 2010, 84, 183-190.

145 S. B. Lee, S.-W. Lee, S. Y. Jeong, G. Yoon, S. J. Cho, S. K. Kim, I.-K. Lee, B.-C. Ahn, J. Lee and Y. H. Jeon, ACS Appl. Mater. Interfaces, 2017, 9, 8480-8489.

146 S. B. Lee, D. Kumar, Y. Li, I.-K. Lee, S. J. Cho, S. K. Kim, S.-W. Lee, S. Y. Jeong, J. Lee and Y. H. Jeon, J. Nanobiotechnol., 2018, 16, 41.

147 L. Zhao, Y. Li, J. Zhu, N. Sun, N. Song, Y. Xing, H. Huang and J. Zhao, J. Nanobiotechnol., 2019, 17, 30.

148 N. Sun, L. Zhao, J. Zhu, Y. Li, N. Song, Y. Xing, W. Qiao, H. Huang and J. Zhao, Int. J. Nanomed., 2019, 14, 4367-4381.

149 J. Lee, T. S. Lee, J. Ryu, S. Hong, M. Kang, K. Im, J. H. Kang, S. M. Lim, S. Park and R. Song, J. Nucl. Med., 2013, 54, 96-103.

150 J. Jun Park, T. Sup Lee, J. Hyun Kang, R. Song and G. Jeong Cheon, Appl. Radiat. Isot., 2011, 69, 56-62.

151 H. Fayez, M. A. El-Motaleb and A. A. Selim, J. Labelled Compd. Radiopharm., 2020, 63, 25-32.

152 N. Malik, R. Wiwattanapatapee, R. Klopsch, K. Lorenz, H. Frey, J. W. Weener, E. W. Meijer, W. Paulus and R. Duncan, J. Controlled Release, 2000, 65, 133-148.

153 W. Yang, R. F. Barth, D. M. Adams, M. J. Ciesielski, R. A. Fenstermaker, S. Shukla, W. Tjarks and M. A. Caligiuri, Cancer Res., 2002, 62, 6552-6558.

154 G. Thiagarajan, S. Sadekar, K. Greish, A. Ray and H. Ghandehari, Mol. Pharmaceutics, 2013, 10, 988-998.

155 S. Sadekar, A. Ray, M. Janàt-Amsbury, C. M. Peterson and H. Ghandehari, Biomacromolecules, 2011, 12, 88-96.

156 R.-Q. Huang, Y.-H. Qu, W.-L. Ke, J.-H. Zhu, Y.-Y. Pei and C. Jiang, FASEB J., 2007, 21, 1117-1125.

157 K. de Winne, S. Vanderkerken, K. Hoste, P. Dubruel, E. Schacht, M. Jelinkova and B. Rihova, J. Bioact. Compat. Polym., 2004, 19, 367-382.

158 S. J. Guillaudeu, M. E. Fox, Y. M. Haidar, E. E. Dy, F. C. Szoka and J. M. J. Fréchet, Bioconjugate Chem., 2008, 19, 461-469.

159 J. Liu, J. Liu, L. Chu, L. Tong, H. Gao, C. Yang, D. Wang, L. Shi, D. Kong and Z. Li, J. Nanosci. Nanotechnol., 2014, 14, 3305-3312.

160 E. R. Gillies, E. Dy, J. M. J. Fréchet and F. C. Szoka, Mol. Pharmaceutics, 2005, 2, 129-138.

161 R. He, H. Wang, Y. Su, C. Chen, L. Xie, L. Chen, J. Yu, Y. Toledo, G. S. Abayaweera, G. Zhu and S. H. Bossmann, RSC Adv., 2017, 7, 16181-16188.

162 N. Nasongkia, B. Chen, N. Macaraeg, M. E. Fox, J. M. J. Fréchet and F. C. Szoka, J. Am. Chem. Soc., 2009, 131, 3842-3843.

163 K. Yang, L. Feng, H. Hong, W. Cai and Z. Liu, Nat. Protoc., 2013, 8, 2392-2403.

164 T. Cao, P. You, X. Zhou, J. Luo, X. Xu, Z. Zhou, S. Yang, Y. Zhang, H. Yang and M. Wang, J. Mater. Chem. B, 2016, 4, 6446-6453.

165 H. Zhao, Y. Chao, J. Liu, J. Huang, J. Pan, W. Guo, J. Wu, M. Sheng, K. Yang, J. Wang and Z. Liu, Theranostics, 2016, 6, 1833-1843. 
166 A. Lalatsa, N. L. Garrett, T. Ferrarelli, J. Moger, A. G. Schätzlein and I. F. Uchegbu, Mol. Pharmaceutics, 2012, 9, 1764-1774.

167 O. Sedláček, J. Kučka and M. Hrubý, Appl. Radiat. Isot., 2015, 95, 129-134.

168 K. Wagener, D. Moderegger, M. Allmeroth, A. Reibel, S. Kramer, B. Biesalski, N. Bausbacher, R. Zentel, O. Thews and F. Rösch, Nucl. Med. Biol., 2018, 58, 59-66.

169 B. Sadarani, A. Majumdar, S. Paradkar, A. Mathur, S. Sachdev, B. Mohanty and P. Chaudhari, Biomed. Pharmacother., 2019, 114, 108770.

170 L. Yang, C. Zhang, J. Liu, F. Huang, Y. Zhang, X. Liang and J. Liu, Adv. Healthcare Mater., 2020, 9, 1901616.

171 C. Zhan, B. Gu, C. Xie, J. Li, Y. Liu and W. Lu, J. Controlled Release, 2010, 143, 136-142.

172 J. Shen, C. Zhan, C. Xie, Q. Meng, B. Gu, C. Li, Y. Zhang and W. Lu, J. Drug Targeting, 2011, 19, 197-203.

173 H.-W. Kao, C.-J. Chan, Y.-C. Chang, Y.-H. Hsu, M. Lu, J. Shian-Jy Wang, Y.-Y. Lin, S.-J. Wang and H.-E. Wang, Appl. Radiat. Isot., 2013, 80, 88-94.

174 Y. Tao, P. Ren, X. Yi, H. Zhou, S. Xiong, F. Ge, L. Chen and K. Yang, Part. Part. Syst. Charact., 2019, 36, 1900018.

175 R. Toita, Y. Kanai, H. Watabe, K. Nakao, S. Yamamoto, J. Hatazawa and M. Akashi, Bioorg. Med. Chem., 2013, 21, 5310-5315.

176 D. G. Colak, I. Cianga, D. O. Demirkol, O. Kozgus, E. I. Medine, S. Sakarya, P. Unak, S. Timur and Y. Yagci, J. Mater. Chem., 2012, 22, 9293-9300.

177 P. P. Di Mauro, Int. J. Pharm., 2018, 553, 169-185.

178 S. Rahmani, C. H. Villa, A. F. Dishman, M. E. Grabowski, D. C. Pan, H. Durmaz, A. C. Misra, L. Colón-Meléndez, M. J. Solomon, V. R. Muzykantov and J. Lahann, J. Drug Targeting, 2015, 23, 750-758.

179 C. Tang, J. Edelstein, J. L. Mikitsh, E. Xiao, A. H. Hemphill, R. Pagels, A. M. Chacko and R. Prud'homme, J. Mater. Chem. B, 2016, 4, 2428-2434.

180 E. A. Simone, B. J. Zern, A. M. Chacko, J. L. Mikitsh, E. R. Blankemeyer, S. Muro, R. V. Stan and V. R. Muzykantov, Biomaterials, 2012, 33, 5406-5413.

181 X. Zhong, K. Yang, Z. Dong, X. Yi, Y. Wang, C. Ge, Y. Zhao and Z. Liu, Adv. Funct. Mater., 2015, 25, 7327-7336.

182 X. Yi, H. Zhou, Z. Zhang, S. Xiong and K. Yang, Biomaterials, 2020, 233, 119764.

183 A. Ji, Y. Zhang, G. Lv, J. Lin, N. Qi, F. Ji and M. Du, J. Labelled Compd. Radiopharm., 2018, 61, 362-369.

184 A. Almutairi, R. Rossin, M. Shokeen, A. Hagooly, A. Ananth, B. Capoccia, S. Guillaudeu, D. Abendschein, C. J. Anderson, M. J. Welch and J. M. J. Fréchet, Proc. Natl. Acad. Sci. U. S. A., 2009, 106, 685-690.

185 Y. Liu, A. Ibricevic, J. A. Cohen, J. L. Cohen, S. P. Gunsten, J. M. J. Fréchet, M. J. Walter, M. J. Welch and S. L. Brody, Mol. Pharmaceutics, 2009, 6, 1891-1902.

186 P. P. Di Mauro, V. Gómez-Vallejo, Z. Baz Maldonado, J. Llop Roig and S. Borrós, Bioconjugate Chem., 2015, 26, 582-592.

187 E. M. El-Marakby, R. M. Hathout, I. Taha, S. Mansour and N. D. Mortada, Int. J. Pharm., 2017, 525, 123-138.
188 O. Akca, P. Unak, E. I. Medine, S. Sakarya, A. Yurt Kilcar, C. Ichedef, R. Bekis and S. Timur, Radiochim. Acta, 2014, 102(9), 849-859, DOI: 10.1515/ract-2013-2152.

189 S. Guerrero, J. R. Herance, S. Rojas, J. F. Mena, J. D. Gispert, G. A. Acosta, F. Albericio and M. J. Kogan, Bioconjugate Chem., 2012, 23, 399-408.

190 J.-P. Meyer, P. Adumeau, J. S. Lewis and B. M. Zeglis, Bioconjugate Chem., 2016, 27, 2791-2807.

191 K. Wagener, M. Worm, S. Pektor, M. Schinnerer, R. Thiermann, M. Miederer, H. Frey and F. Rösch, Biomacromolecules, 2018, 19, 2506-2516.

192 A. T. Reibel, S. S. Müller, S. Pektor, N. Bausbacher, M. Miederer, H. Frey and F. Rösch, Biomacromolecules, 2015, 16, 842-851.

193 E. J. Keliher, Y.-X. Ye, G. R. Wojtkiewicz, A. D. Aguirre, B. Tricot, M. L. Senders, H. Groenen, F. Fay, C. PerezMedina, C. Calcagno, G. Carlucci, T. Reiner, Y. Sun, G. Courties, Y. Iwamoto, H.-Y. Kim, C. Wang, J. W. Chen, F. K. Swirski, H.-Y. Wey, J. Hooker, Z. A. Fayad, W. J. M. Mulder, R. Weissleder and M. Nahrendorf, Nat. Commun., 2017, 8, 14064.

194 J. Jeon, J. A. Kang, H. E. Shim, Y. R. Nam, S. Yoon, H. R. Kim, D. E. Lee and S. H. Park, Bioorg. Med. Chem., 2015, 23, 3303-3308.

195 C.-Y. Wu, J.-J. Lin, W.-Y. Chang, C.-Y. Hsieh, C.-C. Wu, H.S. Chen, H.-J. Hsu, A.-S. Yang, M.-H. Hsu and W.-Y. Kuo, Colloids Surf., B, 2019, 183, 110387.

196 S. Ghiassian, L. Yu, P. Gobbo, A. Nazemi, T. Romagnoli, W. Luo, L. G. Luyt and M. S. Workentin, ACS Omega, 2019, 4, 19106-19115.

197 M. De Simone, D. Panetta, E. Bramanti, C. Giordano, M. C. Salvatici, L. Gherardini, A. Menciassi, S. Burchielli, C. Cinti and P. A. Salvadori, Contrast Media Mol. Imaging, 2016, 11, 561-571.

198 Q. Liu, Y. Sun, C. Li, J. Zhou, C. Li, T. Yang, X. Zhang, T. Yi, D. Wu and F. Li, ACS Nano, 2011, 5, 3146-3157.

199 Y. Sun, M. Yu, S. Liang, Y. Zhang, C. Li, T. Mou, W. Yang, X. Zhang, B. Li, C. Huang and F. Li, Biomaterials, 2011, 32, 2999-3007.

200 J. Zhou, M. Yu, Y. Sun, X. Zhang, X. Zhu, Z. Wu, D. Wu and F. Li, Biomaterials, 2011, 32, 1148-1156.

201 S. Alonso-deCastro, E. Ruggiero, A. Lekuona Fernández, U. Cossío, Z. Baz, D. Otaegui, V. Gómez-Vallejo, D. Padro, J. Llop and L. Salassa, Inorganics, 2019, 7, 60.

202 L. L. Israel, F. Karimi, S. Bianchessi, E. Scanziani, L. Passoni, M. Matteoli, B. Langström and J.-P. Lellouche, Mater. Res. Express, 2015, 2, 095009.

203 T. Tang, Y. Wei, Q. Yang, Y. Yang, M. J. Sailor and H.-B. Pang, Nanoscale, 2019, 11, 22248-22254.

204 X. Sun, X. Huang, J. Guo, W. Zhu, Y. Ding, G. Niu, A. Wang, D. O. Kiesewetter, Z. L. Wang, S. Sun and X. Chen, J. Am. Chem. Soc., 2014, 136, 1706-1709.

205 Y. Sun, X. Zhu, J. Peng and F. Li, ACS Nano, 2013, 7, 11290-11300.

206 B. Roldan Cuenya and F. Behafarid, Surf. Sci. Rep., 2015, 70, 135-187. 
207 M. Králik, Chem. Pap., 2014, 68, 1625-1638, DOI: 10.2478/ s11696-014-0624-9.

208 F. Chen, P. A. Ellison, C. M. Lewis, H. Hong, Y. Zhang, S. Shi, R. Hernandez, M. E. Meyerand, T. E. Barnhart and W. Cai, Angew. Chem., Int. Ed., 2013, 52, 13319-13323.

209 E. Boros, A. M. Bowen, L. Josephson, N. Vasdev and J. P. Holland, Chem. Sci., 2015, 6, 225-236.

210 T. M. Shaffer, S. Harmsen, E. Khwaja, M. F. Kircher, C. M. Drain and J. Grimm, Nano Lett., 2016, 16, 5601-5604.

211 T. M. Shaffer, M. A. Wall, S. Harmsen, V. A. Longo, C. M. Drain, M. F. Kircher and J. Grimm, Nano Lett., $2015,15,864-868$.

212 T. M. Shaffer, S. Harmsen, E. Khwaja, M. F. Kircher, C. M. Drain and J. Grimm, Nano Lett., 2016, 16, 5601-5604.

213 S. Belderbos, M. A. González-Gómez, F. Cleeren, J. Wouters, Y. Piñeiro, C. M. Deroose, A. Coosemans, W. Gsell, G. Bormans, J. Rivas and U. Himmelreich, EJNMMI Res., 2020, 10, 73.

214 M. A. González-Gómez, S. Belderbos, S. Yañez-Vilar, Y. Piñeiro, F. Cleeren, G. Bormans, C. M. Deroose, W. Gsell, U. Himmelreich and J. Rivas, Nanomaterials, 2019, 9, 1626.

215 X. Cui, S. Belo, D. Krüger, Y. Yan, R. T. M. de Rosales, M. Jauregui-Osoro, H. Ye, S. Su, D. Mathe, N. Kovács, I. Horváth, M. Semjeni, K. Sunassee, K. Szigeti, M. A. Green and P. J. Blower, Biomaterials, 2014, 35, 5840-5846.

216 B. T. Cisneros, J. J. Law, M. L. Matson, A. Azhdarinia, E. M. Sevick-Muraca and L. J. Wilson, Nanomedicine, 2014, 9, 2499-2509.

217 F. Man, P. J. Gawne and R. T. M. de Rosales, Adv. Drug Delivery Rev., 2019, 143, 134-160.

218 J. Marik, M. S. Tartis, H. Zhang, J. Y. Fung, A. Kheirolomoom, J. L. Sutcliffe and K. W. Ferrara, Nucl. Med. Biol., 2007, 34, 165-171.

219 J. W. Seo, L. M. Mahakian, S. Tam, S. Qin, E. S. Ingham, C. F. Meares and K. W. Ferrara, Nucl. Med. Biol., 2015, 42, 155-163.

220 P. Laverman, E. T. M. Dams, W. J. G. Oyen, G. Storm, E. B. Koenders, R. Prevost, J. W. M. van der Meer, F. H. M. Corstens and O. C. Boerman, J. Nucl. Med., 1999, 40, 192-197.

221 V. J. Richardson, K. Jeyasingh, R. F. Jewkes, B. E. Ryman and M. H. Tattersall, Biochem. Soc. Trans., 1977, 5, 290-291.

222 V. J. Richardson, K. Jeyasingh, R. F. Jewkes, B. E. Ryman and M. H. Tattersall, J. Nucl. Med., 1978, 19, 1049-1054.

223 V. J. Richardson, B. E. Ryman, R. F. Jewkes, M. H. Tattersall and E. S. Newlands, Int. J. Nucl. Med. Biol., 1978, 5, 118, 121-122, 123.

224 V. J. Richardson, B. E. Ryman, R. F. Jewkes, K. Jeyasingh, M. N. H. Tattersall, E. S. Newlands and S. B. Kaye, Br. J. Cancer, 1979, 40, 35-43.

225 M. P. Osborne, J. H. Payne, V. J. Richardson, V. R. McCready and B. E. Ryman, Br. J. Surg., 1983, 70, 141-144.

226 D. S. Abou, D. L. J. Thorek, N. N. Ramos, M. W. H. Pinkse, H. T. Wolterbeek, S. D. Carlin, B. J. Beattie and J. S. Lewis, Pharm. Res., 2013, 30, 878-888.
227 H. Zhang, J. Kusunose, A. Kheirolomoom, J. W. Seo, J. Qi, K. D. Watson, H. A. Lindfors, E. Ruoslahti, J. L. Sutcliffe and K. W. Ferrara, Biomaterials, 2008, 29, 1976-1988.

228 H. Zhang, N. Li, P. Sirish, L. Mahakian, E. Ingham, F.-R. Curry, S. Yamada, N. Chiamvimonvat and K. W. Ferrara, J. Controlled Release, 2012, 163, 10-17.

229 F. Emmetiere, C. Irwin, N. T. Viola-Villegas, V. Longo, S. M. Cheal, P. Zanzonico, N. Pillarsetty, W. A. Weber, J. S. Lewis and T. Reiner, Bioconjugate Chem., 2013, 24, 1784-1789.

230 A. T. I. Jensen, T. Binderup, T. L. Andresen, A. Kjær and P. H. Rasmussen, J. Liposome Res., 2012, 22, 295-305.

231 T. Urakami, S. Akai, Y. Katayama, N. Harada, H. Tsukada and N. Oku, J. Med. Chem., 2007, 50, 6454-6457.

232 T. Urakami, A. T. Kawaguchi, S. Akai, K. Hatanaka, H. Koide, K. Shimizu, T. Asai, D. Fukumoto, N. Harada, H. Tsukada and N. Oku, Artif. Organs, 2009, 33, 164-168.

233 N. Oku, M. Yamashita, Y. Katayama, T. Urakami, K. Hatanaka, K. Shimizu, T. Asai, H. Tsukada, S. Akai and H. Kanazawa, Int. J. Pharm., 2011, 403, 170-177.

234 T. Fukuta, T. Ishii, T. Asai, G. Nakamura, Y. Takeuchi, A. Sato, Y. Agato, K. Shimizu, S. Akai, D. Fukumoto, N. Harada, H. Tsukada, A. T. Kawaguchi and N. Oku, Artif. Organs, 2014, 38, 662-666.

235 A. T. Reibel, S. S. Müller, S. Pektor, N. Bausbacher, M. Miederer, H. Frey and F. Rösch, Biomacromolecules, 2015, 16, 842-851.

236 K. Wagener, M. Worm, S. Pektor, M. Schinnerer, R. Thiermann, M. Miederer, H. Frey and F. Rösch, Biomacromolecules, 2018, 19, 2506-2516.

237 D. J. Hnatowich, B. Friedman, B. Clancy and M. Novak, J. Nucl. Med., 1981, 22, 810-814.

238 Q. F. Ahkong and C. Tilcock, Int. J. Radiat. Appl. Instrum., Part B, 1992, 19, 831-840.

239 C. Tilcock, Q. F. Ahkong and D. Fisher, Nucl. Med. Biol., 1994, 21, 89-96.

240 C. Tilcock, Q. F. Ahkong and D. Fisher, Biochim. Biophys. Acta, 1993, 1148, 77-84.

241 H. Hwang, H.-S. H.-J. Jeong, P.-S. Oh, M. Kim, T.-K. Lee, J. Kwon, H.-S. Kim, S. T. Lim, M.-H. Sohn and H.-S. H.-J. Jeong, Nucl. Med. Biol., 2016, 43, 552-558.

242 L. O. F. Monteiro, R. S. Fernandes, C. M. R. Oda, S. C. Lopes, D. M. Townsend, V. N. Cardoso, M. C. Oliveira, E. A. Leite, D. Rubello and A. L. B. de Barros, Biomed. Pharmacother., 2018, 97, 489-495.

243 A. T. M. e Silva, A. L. C. Maia, J. de Oliveira Silva, A. L. B. de Barros, D. C. F. Soares, M. T. Q. de Magalhães, R. José Alves and G. A. Ramaldes, Carbohydr. Res., 2018, 465, 52-57.

244 A. Helbok, C. Decristoforo, G. Dobrozemsky, C. Rangger, E. Diederen, B. Stark, R. Prassl and E. von Guggenberg, J. Liposome Res., 2010, 20, 219-227.

245 E. T. Dams, M. M. Reijnen, W. J. Oyen, O. C. Boerman, P. Laverman, G. Storm, J. W. van der Meer, F. H. Corstens and H. van Goor, Ann. Surg., 1999, 229, 551-557.

246 C. J. J. M. Sikkink, M. M. P. J. Reijnen, P. Laverman, W. J. G. Oyen and H. van Goor, J. Surg. Res., 2009, 154, 246-251. 
247 A. Brouwers, D. De Jong, E. Dams, W. Oyen, O. Boerman, P. Laverman, T. Naber, G. Storm and F. Corstens, J. Drug Targeting, 2000, 8, 225-233.

248 Z. Varga, I. C. Szigyártó, I. Gyurkó, R. Dóczi, I. Horváth, D. Máthé and K. Szigeti, Contrast Media Mol. Imaging, 2017, 2017, 1-8.

249 T. A. Elbayoumi and V. P. Torchilin, Eur. J. Nucl. Med. Mol. Imaging, 2006, 33, 1196-1205.

250 E. Jestin, M. Mougin-Degraef, A. Faivre-Chauvet, P. Remaud-Le Saëc, F. Hindre, J. P. Benoit, J. F. Chatal, J. Barbet and J. F. Gestin, Q. J. Nucl. Med. Mol. Imaging, 2007, 51, 51-60.

251 M. Mougin-Degraef, C. Bourdeau, E. Jestin, C. Saï-Maurel, M. Bourgeois, P. R.-L. Saëc, P. Thédrez, J.-F. Gestin, J. Barbet and A. Faivre-Chauvet, Int. J. Pharm., 2007, 344, 110-117.

252 Y. Duan, L. Wei, J. Petryk and T. Ruddy, Int. J. Nanomed., 2016, 11, 5697-5708.

253 A. K. Iyer, Y. Su, J. Feng, X. Lan, X. Zhu, Y. Liu, D. Gao, Y. Seo, H. F. VanBrocklin, V. Courtney Broaddus, B. Liu and J. He, Biomaterials, 2011, 32, 2605-2613.

254 T. van der Geest, P. Laverman, D. Gerrits, G. M. Franssen, J. M. Metselaar, G. Storm and O. C. Boerman, J. Controlled Release, 2015, 220, 239-244.

255 E. D. Hood, C. F. Greineder, T. Shuvaeva, L. Walsh, C. H. Villa and V. R. Muzykantov, Bioconjugate Chem., 2018, 29, 3626-3637.

256 J. Malinge, B. Géraudie, P. Savel, V. Nataf, A. Prignon, C. Provost, Y. Zhang, P. Ou, K. Kerrou, J.-N. Talbot, J.-M. Siaugue, M. Sollogoub and C. Ménager, Mol. Pharmaceutics, 2017, 14, 406-414.

257 J. W. Seo, H. Zhang, D. L. Kukis, C. F. Meares and K. W. Ferrara, Bioconjugate Chem., 2008, 19, 2577-2584.

258 E. E. Paoli, D. E. Kruse, J. W. Seo, H. Zhang, A. Kheirolomoom, K. D. Watson, P. Chiu, H. Stahlberg and K. W. Ferrara, J. Controlled Release, 2010, 143, 13-22.

259 C. B. Rygh, S. Qin, J. W. Seo, L. M. Mahakian, H. Zhang, R. Adamson, J. Q. Chen, A. D. Borowsky, R. D. Cardiff, R. K. Reed, F.-R. E. Curry and K. W. Ferrara, Clin. Cancer Res., 2011, 17, 550-559.

260 L. M. Mahakian, D. G. Farwell, H. Zhang, J. W. Seo, B. Poirier, S. P. Tinling, A. M. Afify, E. M. Haynam, D. Shaye and K. W. Ferrara, Mol. Imaging Biol., 2014, 16, 284-292.

261 A. W. Wong, E. Ormsby, H. Zhang, J. W. Seo, L. M. Mahakian, C. F. Caskey and K. W. Ferrara, Am. J. Nucl. Med. Mol. Imaging, 2013, 3, 32-43.

262 J. W. Seo, L. M. Mahakian, A. Kheirolomoom, H. Zhang, C. F. Meares, R. Ferdani, C. J. Anderson and K. W. Ferrara, Bioconjugate Chem., 2010, 21, 1206-1215.

263 J. W. Seo, S. Qin, L. M. Mahakian, K. D. Watson, A. Kheirolomoom and K. W. Ferrara, J. Controlled Release, 2011, 151, 28-34.

264 S. Lee, K. Gangangari, T. M. Kalidindi, B. Punzalan, S. M. Larson and N. V. K. Pillarsetty, Nucl. Med. Biol., 2016, 43, 781-787.
265 N. Mitchell, T. L. Kalber, M. S. Cooper, K. Sunassee, S. L. Chalker, K. P. Shaw, K. L. Ordidge, A. Badar, S. M. Janes, P. J. Blower, M. F. Lythgoe, H. C. Hailes and A. B. Tabor, Biomaterials, 2013, 34, 1179-1192.

266 C. M. Kang, H. J. Koo, S. Lee, K. C. Lee, Y. K. Oh and Y. S. Choe, Nucl. Med. Biol., 2013, 40, 1018-1024.

267 A. I. Jensen, G. W. Severin, A. E. Hansen, F. P. Fliedner, R. Eliasen, L. Parhamifar, A. Kjær, T. L. Andresen and J. R. Henriksen, J. Controlled Release, 2018, 269, 100-109.

268 S. McQuarrie, J. Mercer, A. Syme, M. Suresh and G. Miller, J. Pharm. Pharm. Sci., 2005, 7, 29-34.

269 S. W. Zielhuis, J.-H. Seppenwoolde, V. A. P. Mateus, C. J. G. Bakker, G. C. Krijger, G. Storm, B. A. Zonnenberg, A. D. van het Schip, G. A. Koning and J. F. W. Nijsen, Cancer Biother. Radiopharm., 2006, 21, 520-527.

270 C. Perez-Medina, D. Abdel-Atti, Y. Zhang, V. A. Longo, C. P. Irwin, T. Binderup, J. Ruiz-Cabello, Z. A. Fayad, J. S. Lewis, W. J. M. Mulder and T. Reiner, J. Nucl. Med., 2014, 55, 1706-1711.

271 C. Pérez-Medina, D. Abdel-Atti, J. Tang, Y. Zhao, Z. A. Fayad, J. S. Lewis, W. J. M. Mulder and T. Reiner, Nat. Commun., 2016, 7, 11838.

272 G. Srimathveeravalli, D. Abdel-Atti, C. Pérez-Medina, H. Takaki, S. B. Solomon, W. J. M. Mulder and T. Reiner, Mol. Imaging, 2018, 17, DOI: 10.1177/1536012117749726.

273 M. E. Lobatto, T. Binderup, P. M. Robson, L. F. P. Giesen, C. Calcagno, J. Witjes, F. Fay, S. Baxter, C. H. Wessel, M. Eldib, J. Bini, S. D. Carlin, E. S. G. Stroes, G. Storm, A. Kjaer, J. S. Lewis, T. Reiner, Z. A. Fayad, W. J. M. Mulder and C. Pérez-Medina, Bioconjugate Chem., 2020, 31, 360-368.

274 B. Yu, S. Goel, D. Ni, P. A. Ellison, C. M. Siamof, D. Jiang, L. Cheng, L. Kang, F. Yu, Z. Liu, T. E. Barnhart, Q. He, H. Zhang and W. Cai, Adv. Mater., 2018, 30, 1704934.

275 V. J. Caride and B. L. Zaret, Science, 1977, 198, 735-738.

276 T. N. Palmer, V. J. Caride, L. A. Fernandez and J. Twickler, Biosci. Rep., 1981, 1, 337-344.

277 T. N. Palmer and J. P. Warner, Biosci. Rep., 1983, 3, 479-485.

278 N. Oku, Y. Namba, A. Takeda and S. Okada, Nucl. Med. Biol., 1993, 20, 407-412.

279 M. L. Corvo, O. C. Boerman, W. J. G. Oyen, J. C. S. Jorge, M. E. M. Cruz, D. J. A. Crommelin and G. Storm, Pharm. Res., 2000, 17, 600-606.

280 D. C. F. Soares, M. C. de Oliveira, R. G. dos Santos, M. S. Andrade, J. M. C. Vilela, V. N. Cardoso and G. A. Ramaldes, Eur. J. Pharm. Sci., 2011, 42, 462-469.

281 S. Sofou, J. L. Thomas, H. Lin, M. R. McDevitt, D. A. Scheinberg and G. Sgouros, J. Nucl. Med., 2004, 45, 253-260.

282 M. R. Mauk and R. C. Gamble, Anal. Biochem., 1979, 94, 302-307.

283 R. T. Proffitt, L. E. Williams, C. A. Presant, G. W. Tin, J. A. Uliana, R. C. Gamble and J. D. Baldeschwieler, J. Nucl. Med., 1983, 24, 45-51.

284 K. J. Hwang, J. Nucl. Med., 1978, 19, 1162-1170. 
285 P. L. Beaumier and K. J. Hwang, Biochim. Biophys. Acta, 1983, 731, 23-30.

286 P. L. Beaumier and K. J. Hwang, J. Nucl. Med., 1982, 23, 810-815.

287 H. Essien and K. J. Hwang, Biochim. Biophys. Acta, 1988, 944, 329-336.

288 O. C. Boerman, G. Storm, W. J. G. Oyen, L. van Bloois, J. W. M. van der Meer, R. A. M. J. Claessens, D. J. A. Crommelin and F. H. M. Corstens, J. Nucl. Med., 1995, 36, 1639-1644.

289 N. Postma, O. Boerman, W. J. Oyen, J. Zuidema and G. Storm, J. Controlled Release, 1999, 58, 51-60.

290 S. Stewart and K. J. Harrington, Oncology, 1997, 11, 33-37.

291 K. J. Harrington, G. Rowlinson-Busza, K. N. Syrigos, P. S. Uster, R. M. Abra and J. S. W. Stewart, Br. J. Cancer, 2000, 83, 232-238.

292 K. J. Harrington, G. Rowlinson-Busza, K. N. Syrigos, P. S. Uster, R. G. Vile and J. S. W. Stewart, Clin. Cancer Res., 2000, 6, 2528-2537.

293 M. L. Corvo, O. C. Boerman, W. J. Oyen, L. Van Bloois, M. E. M. Cruz, D. J. Crommelin and G. Storm, Biochim. Biophys. Acta, Biomembr., 1999, 1419, 325-334.

294 D. Utkhede, V. Yeh, M. Szucs and C. Tilcock, J. Liposome Res., 1994, 4, 1049-1061.

295 A. Gabizon, J. Hliberty, R. M. Straubinger, D. C. Price and D. Papahadjopoulos, J. Liposome Res., 1988, 1, 123-135.

296 A. Gabizon, D. C. Price, J. Huberty, R. S. Bresalier and D. Papahadjopoulos, Cancer Res., 1990, 50, 6371-6378.

297 H.-E. Wang, H.-M. Yu, Y.-C. Lu, N.-N. Heish, Y.-L. Tseng, K.-L. Huang, K.-T. Chuang, C.-H. Chen, J.-J. Hwang, W.J. Lin, S.-J. Wang, G. Ting, J. Whang-Peng and W.-P. Deng, Nucl. Instrum. Methods Phys. Res., Sect. A, 2006, 569, 533-537.

298 A. L. Petersen, T. Binderup, P. Rasmussen, J. R. Henriksen, D. R. Elema, A. Kjær and T. L. Andresen, Biomaterials, 2011, 32, 2334-2341.

299 A. L. Petersen, T. Binderup, R. I. Jølck, P. Rasmussen, J. R. Henriksen, A. K. Pfeifer, A. Kjær and T. L. Andresen, J. Controlled Release, 2012, 160, 254-263.

300 L. W. Locke, M. W. Mayo, A. D. Yoo, M. B. Williams and S. S. Berr, Biomaterials, 2012, 33, 7785-7793.

301 M.-Y. Chang, J. Seideman and S. Sofou, Bioconjugate Chem., 2008, 19, 1274-1282.

302 A. Bandekar, C. Zhu, R. Jindal, F. Bruchertseifer, A. Morgenstern and S. Sofou, J. Nucl. Med., 2014, 55, 107-114.

303 N. Li, Z. Yu, T. Pham, P. Blower and R. Yan, Int. J. Nanomed., 2017, 12, 3281-3294.

304 S. Edmonds, A. Volpe, H. Shmeeda, A. C. Parente-Pereira, R. Radia, J. Baguña-Torres, I. Szanda, G. W. Severin, L. Livieratos, P. J. Blower, J. Maher, G. O. Fruhwirth, A. Gabizon and R. T. M. de Rosales, ACS Nano, 2016, 10, 10294-10307.

305 P. J. Gawne, F. Clarke, K. Turjeman, A. P. Cope, N. J. Long, Y. Barenholz, S. Y. A. Terry and R. T. M. de Rosales, Theranostics, 2020, 10, 3867-3879.
306 P. Gawne, F. Man, J. Fonslet, R. Radia, J. Bordoloi, M. Cleveland, P. Jimenez-Royo, A. Gabizon, P. J. Blower, N. Long and R. T. M. De Rosales, Dalton Trans., 2018, 47, 9283-9293.

307 F. Man, L. Lim, A. Volpe, A. Gabizon, H. Shmeeda, B. Draper, A. C. Parente-Pereira, J. Maher, P. J. Blower, G. O. Fruhwirth and R. T. M. de Rosales, Mol. Ther., 2019, 27, 219-229.

308 J. R. Henriksen, A. L. Petersen, A. E. Hansen, C. G. Frankær, P. Harris, D. R. Elema, A. T. Kristensen, A. Kjær and T. L. Andresen, ACS Appl. Mater. Interfaces, 2015, 7, 22796-22806.

309 A. E. Hansen, A. L. Petersen, J. R. Henriksen, B. Boerresen, P. Rasmussen, D. R. Elema, P. M. af Rosenschöld, A. T. Kristensen, A. Kjær and T. L. Andresen, ACS Nano, 2015, 9, 6985-6995.

310 B. Børresen, J. R. Henriksen, G. Clergeaud, J. S. Jørgensen, F. Melander, D. R. Elema, J. Szebeni, S. A. Engelholm, A. T. Kristensen, A. Kjær, T. L. Andresen and A. E. Hansen, ACS Nano, 2018, 12, 11386-11398.

311 A. E. Hansen, F. P. Fliedner, J. R. Henriksen, J. T. Jørgensen, A. E. Clemmensen, B. Børresen, D. R. Elema, A. Kjær and T. L. Andresen, Nanomedicine, 2018, 14, 27-34.

312 A. S. Rudolph, R. W. Klipper, B. Goins and W. T. Phillips, Proc. Natl. Acad. Sci. U. S. A., 1991, 88, 10976-10980.

313 W. T. Phillips, A. S. Rudolph, B. Goins, J. H. Timmons, R. Klipper and R. Blumhardt, Int. J. Radiat. Appl. Instrum., Part B, 1992, 19, 539-547.

314 M. Suresh and Y. Cao, J. Pharm. Pharm. Sci., 1998, 1, 31-37.

315 A. Bao, B. Goins, R. Klipper, G. Negrete, M. Mahindaratne and W. T. Phillips, J. Pharm. Sci., 2003, 92, 1893-1904.

316 S. Li, B. Goins, W. T. Phillips and A. Bao, J. Liposome Res., 2011, 21, 17-27.

317 A. Bao, J. Pharmacol. Exp. Ther., 2003, 308, 419-425.

318 A. Bao, B. Goins, R. Klipper, G. Negrete and W. T. Phillips, J. Nucl. Med., 2003, 44, 1992-1999.

319 A. Soundararajan, A. Bao, W. T. Phillips, R. Perez and B. A. Goins, Nucl. Med. Biol., 2009, 36, 515-524.

320 L.-C. Chen, Y.-H. Wu, I.-H. Liu, C.-L. Ho, W.-C. Lee, C.H. Chang, K.-L. Lan, G. Ting, T.-W. Lee and J.-H. Shien, Nucl. Med. Biol., 2012, 39, 35-43.

321 C.-W. Hsu, Y.-J. Chang, C.-H. Chang, L.-C. Chen, K.-L. Lan, G. Ting and T.-W. Lee, Cancer Biother. Radiopharm., 2012, 27, 481-489.

322 L.-T. Lin, C.-H. Chang, H.-L. Yu, R.-S. Liu, H.-E. Wang, S.J. Chiu, F.-D. Chen, T.-W. Lee and Y.-J. Lee, J. Nucl. Med., 2014, 55, 1864-1870.

323 W.-C. Hsu, C.-N. Cheng, T.-W. Lee and J.-J. Hwang, Anticancer Res., 2015, 35, 4777-4788.

324 H. Lee, J. Zheng, D. Gaddy, K. D. Orcutt, S. Leonard, E. Geretti, J. Hesterman, C. Harwell, J. Hoppin, D. A. Jaffray, T. Wickham, B. S. Hendriks and D. Kirpotin, Nanomedicine, 2015, 11, 155-165.

325 S. J. Blocker, K. A. Douglas, L. A. Polin, H. Lee, B. S. Hendriks, E. Lalo, W. Chen and A. F. Shields, Theranostics, 2017, 7, 4229-4239. 
326 H. Lee, A. F. Shields, B. A. Siegel, K. D. Miller, I. Krop, C. X. Ma, P. M. LoRusso, P. N. Munster, K. Campbell, D. F. Gaddy, S. C. Leonard, E. Geretti, S. J. Blocker, D. B. Kirpotin, V. Moyo, T. J. Wickham and B. S. Hendriks, Clin. Cancer Res., 2017, 23, 4190-4202.

327 H. Lee, D. Gaddy, M. Ventura, N. Bernards, R. de Souza, D. Kirpotin, T. Wickham, J. Fitzgerald, J. Zheng and B. S. Hendriks, Theranostics, 2018, 8, 2300-2312.

328 G. Engudar, H. Schaarup-Jensen, F. P. Fliedner, A. E. Hansen, P. Kempen, R. I. Jølck, A. Kjæer, T. L. Andresen, M. H. Clausen, A. I. Jensen and J. R. Henriksen, Theranostics, 2018, 8, 5828-5841.

329 W. G. Love, N. Amos, B. D. Williams and I. W. Kellaway, J. Microencapsulation, 1989, 6, 105-113.

330 B. C. L. Cheung, T. H. T. Sun, J. M. Leenhouts and P. R. Cullis, Biochim. Biophys. Acta, Biomembr., 1998, 1414, 205-216.

331 S. A. Abraham, K. Edwards, G. Karlsson, S. MacIntosh, L. D. Mayer, C. McKenzie and M. B. Bally, Biochim. Biophys. Acta, Biomembr., 2002, 1565, 41-54.

332 R. Chakravarty, S. Chakraborty and A. Dash, Mol. Pharmaceutics, 2016, 13, 3601-3612.

333 S. A. Graves, R. Hernandez, J. Fonslet, C. G. England, H. F. Valdovinos, P. A. Ellison, T. E. Barnhart, D. R. Elema, C. P. Theuer, W. Cai, R. J. Nickles and G. W. Severin, Bioconjugate Chem., 2015, 26, 2118-2124.

334 D. S. Abou, T. Ku and P. M. Smith-Jones, Nucl. Med. Biol., 2011, 38, 675-681.

335 V. Oliveri and G. Vecchio, Eur. J. Med. Chem., 2016, 120, 252-274.

336 R. Hueting, V. Kersemans, B. Cornelissen, M. Tredwell, K. Hussien, M. Christlieb, A. D. Gee, J. Passchier, S. C. Smart, J. R. Dilworth, V. Gouverneur and R. J. Muschel, J. Nucl. Med., 2014, 55, 128-134.

337 J. J. Bartnicka and P. J. Blower, J. Nucl. Med., 2018, 59, 1355-1359.

338 G. Raposo and W. Stoorvogel, J. Cell Biol., 2013, 200, 373-383.

339 O. M. Elsharkasy, J. Z. Nordin, D. W. Hagey, O. G. de Jong, R. M. Schiffelers, S. E. L. Andaloussi and P. Vader, $A d v$. Drug Delivery Rev., 2020, 159, 332-343, DOI: 10.1016/ j.addr.2020.04.004.

340 A. Khan, F. Man, F. Faruqu, J. Kim, F. Al-Salemee, A. Volpe, G. O. Fruhwirth, K. T. Al-Jamal and R. T. M. de Rosales, ChemRxiv, 2020, DOI: 10.26434/chemrxiv.12730463.v1.

341 A. Banerjee, V. Alves, T. Rondão, J. Sereno, Â. Neves, M. Lino, A. Ribeiro, A. J. Abrunhosa and L. S. Ferreira, Nanoscale, 2019, 11, 13243-13248.

342 F. Royo, U. Cossío, A. Ruiz de Angulo, J. Llop and J. M. Falcon-Perez, Nanoscale, 2019, 11, 1531-1537.

343 F. N. Faruqu, J. T. W. Wang, L. Xu, L. McNickle, E. M. Y. Chong, A. Walters, M. Gurney, A. Clayton, L. A. Smyth, R. Hider, J. Sosabowski and K. T. Al-Jamal, Theranostics, 2019, 9, 1666-1682.

344 S. Shi, T. Li, X. Wen, S. Y. Wu, C. Xiong, J. Zhao, V. R. Lincha, D. S. Chow, Y. Liu, A. K. Sood and C. Li,
Bioconjugate Chem., 2019, 30(10), 2675-2683, DOI: 10.1021/ acs.bioconjchem.9b00587.

345 M. Morishita, Y. Takahashi, M. Nishikawa, K. Sano, K. Kato, T. Yamashita, T. Imai, H. Saji and Y. Takakura, J. Pharm. Sci., 2015, 104, 705-713.

346 A. Matsumoto, Y. Takahashi, M. Nishikawa, K. Sano, M. Morishita, C. Charoenviriyakul, H. Saji and Y. Takakura, Cancer Sci., 2017, 108, 1803-1810.

347 M. H. Rashid, T. F. Borin, R. Ara, K. Angara, J. Cai, B. R. Achyut, Y. Liu and A. S. Arbab, Nanomedicine, 2019, 21, 102072.

348 Z. Varga, I. Gyurkó, K. Pálóczi, E. I. Buzás, I. Horváth, N. Hegedüs, D. Máthé and K. Szigeti, Cancer Biother. Radiopharm., 2016, 31, 168-173.

349 P. Gangadaran, C. M. Hong, J. M. Oh, R. L. Rajendran, S. Kalimuthu, S. H. Son, A. Gopal, L. Zhu, S. H. Baek, S. Y. Jeong, S.-W. Lee, J. Lee and B.-C. Ahn, Front. Pharmacol., 2018, 9, 817.

350 S. H. Son, J. M. Oh, P. Gangadaran, H. D. Ji, H. W. Lee, R. L. Rajendran, S. H. Baek, A. Gopal, S. Kalimuthu, S. Y. Jeong, S. W. Lee, J. Lee and B. C. Ahn, Blood Cells, Mol., Dis., 2020, 80, 102375.

351 M. I. González, P. Martín-Duque, M. Desco and B. Salinas, Nanomaterials, 2020, 10, 1062.

352 T. Smyth, M. Kullberg, N. Malik, P. Smith-Jones, M. W. Graner and T. J. Anchordoquy, J. Controlled Release, 2015, 199, 145-155.

353 D. W. Hwang, H. Choi, S. C. Jang, M. Y. Yoo, J. Y. Park, N. E. Choi, H. J. Oh, S. Ha, Y. S. Lee, J. M. Jeong, Y. S. Gho and D. S. Lee, Sci. Rep., 2015, 5, 1-10.

354 W. Lohcharoenkal, L. Wang, Y. C. Chen and Y. Rojanasakul, BioMed Res. Int., 2014, 2014, 180549.

355 M. J. Hawkins, P. Soon-Shiong and N. Desai, Adv. Drug Delivery Rev., 2008, 60, 876-885.

356 S. Jain, R. Mathur, M. Das, N. K. Swarnakar and A. K. Mishra, Nanomedicine, 2011, 6, 1733-1754.

357 A. Bunschoten, T. Buckle, J. Kuil, G. D. Luker, K. E. Luker, O. E. Nieweg and F. W. B. van Leeuwen, Biomaterials, 2012, 33, 867-875.

358 Y. Yang, T. Neef, C. Mittelholzer, E. Garcia Garayoa, P. Bläuenstein, R. Schibli, U. Aebi and P. Burkhard, J. Nanobiotechnol., 2013, 11, 36.

359 M. Liang, H. Tan, J. Zhou, T. Wang, D. Duan, K. Fan, J. He, D. Cheng, H. Shi, H. S. Choi and X. Yan, ACS Nano, 2018, 12, 9300-9308.

360 S. G. Yang, J. E. Chang, B. Shin, S. Park, K. Na and C. K. Shim, J. Mater. Chem., 2010, 20, 9042-9046.

361 A. Ozgur, F. Y. Lambrecht, K. Ocakoglu, C. Gunduz and M. Yucebas, Int. J. Pharm., 2012, 422, 472-478.

362 A. Woods, A. Patel, D. Spina, Y. Riffo-Vasquez, A. BabinMorgan, R. T. M. De Rosales, K. Sunassee, S. Clark, H. Collins, K. Bruce, L. A. Dailey and B. Forbes, J. Controlled Release, 2015, 210, 1-9.

363 A. G. Gil, J. M. Irache, I. Peñuelas, C. J. González Navarro and A. López de Cerain, Food Chem. Toxicol., 2017, 106, 477-486. 
364 C. Perez-Medina, J. Tang, D. Abdel-Atti, B. Hogstad, M. Merad, E. A. Fisher, Z. A. Fayad, J. S. Lewis, W. J. M. Mulder and T. Reiner, J. Nucl. Med., 2015, 56, 1272-1277.

365 C. Pérez-Medina, T. Binderup, M. E. Lobatto, J. Tang, C. Calcagno, L. Giesen, C. H. Wessel, J. Witjes, S. Ishino, S. Baxter, Y. Zhao, S. Ramachandran, M. Eldib, B. L. Sánchez-Gaytán, P. M. Robson, J. Bini, J. F. Granada, K. M. Fish, E. S. G. Stroes, R. Duivenvoorden, S. Tsimikas, J. S. Lewis, T. Reiner, V. Fuster, A. Kjær, E. A. Fisher, Z. A. Fayad and W. J. M. Mulder, JACC: Cardiovasc. Imaging, 2016, 9, 950-961.

366 J. Tang, S. Baxter, A. Menon, A. Alaarg, B. L. SanchezGaytan, F. Fay, Y. Zhao, M. Ouimet, M. S. Braza, V. A. Longo, D. Abdel-Atti, R. Duivenvoorden, C. Calcagno, G. Storm, S. Tsimikas, K. J. Moore, F. K. Swirski, M. Nahrendorf, E. A. Fisher, C. Pérez-Medina, Z. A. Fayad, T. Reiner and W. J. M. Mulder, Proc. Natl. Acad. Sci. U. S. A., 2016, 113, E6731-E6740.

367 K. H. Zheng, F. M. van der Valk, L. P. Smits, M. Sandberg, J. L. Dasseux, R. Baron, R. Barbaras, C. Keyserling, B. F. Coolen, A. J. Nederveen, H. J. Verberne, T. E. Nell, D. J. Vugts, R. Duivenvoorden, Z. A. Fayad, W. J. M. Mulder, G. A. M. S. van Dongen and E. S. G. Stroes, Atherosclerosis, 2016, 251, 381-388.

368 M. Lameijer, T. Binderup, M. M. T. Van Leent, M. L. Senders, F. Fay, J. Malkus, B. L. Sanchez-Gaytan, A. J. P. Teunissen, N. Karakatsanis, P. Robson, X. Zhou, Y. Ye, G. Wojtkiewicz, J. Tang, T. T. P. Seijkens, J. Kroon, E. S. G. Stroes, A. Kjaer, J. Ochando, T. Reiner, C. PérezMedina, C. Calcagno, E. A. Fischer, B. Zhang, R. E. Temel, F. K. Swirski, M. Nahrendorf, Z. A. Fayad, E. Lutgens, W. J. M. Mulder and R. Duivenvoorden, Nat. Biomed. Eng., 2018, 2, 279-292.

369 T. Binderup, R. Duivenvoorden, F. Fay, M. M. T. Van Leent, J. Malkus, S. Baxter, S. Ishino, Y. Zhao, B. Sanchez-Gaytan, A. J. P. Teunissen, Y. C. A. Frederico, J. Tang, G. Carlucci, S. Lyashchenko, C. Calcagno, N. Karakatsanis, G. Soultanidis, M. L. Senders, P. M. Robson, V. Mani, S. Ramachandran, M. E. Lobatto, B. A. Hutten, J. F. Granada, T. Reiner, F. K. Swirski, M. Nahrendorf, A. Kjaer, E. A. Fisher, Z. A. Fayad, C. Pérez-Medina and W. J. M. Mulder, Sci. Transl. Med., 2019, 11(506), eaaw7736, DOI: $10.1126 /$ scitranslmed.aaw7736.

370 C. A. Mason, S. Kossatz, L. M. Carter, G. Pirovano, C. Brand, N. Guru, C. Pérez-Medina, J. S. Lewis, W. J. M. Mulder and T. Reiner, J. Nucl. Med., 2020, 61, 433-436.

371 M. Wu, J. Shi, D. Fan, Q. Zhou, F. Wang, Z. Niu and Y. Huang, Biomacromolecules, 2013, 14, 4032-4037.

372 P. Kothari, B. P. De, B. He, A. Chen, M. J. Chiuchiolo, D. Kim, A. Nikolopoulou, A. Amor-Coarasa, J. P. Dyke, H. U. Voss, S. M. Kaminsky, C. P. Foley, S. Vallabhajosula, B. Hu, S. G. DiMagno, D. Sondhi, R. G. Crystal, J. W. Babich and D. Ballon, Sci. Rep., 2017, 7, 39594.

373 J. W. Seo, E. S. Ingham, L. Mahakian, S. Tumbale, B. Wu, S. Aghevlian, S. Shams, M. Baikoghli, P. Jain, X. Ding,
N. Goeden, T. Dobreva, N. C. Flytzanis, M. Chavez, K. Singhal, R. Leib, M. L. James, D. J. Segal, R. H. Cheng, E. A. Silva, V. Gradinaru and K. W. Ferrara, Nat. Commun., 2020, 11, 2102.

374 C. I. Øie, D. L. Wolfson, T. Yasunori, G. Dumitriu, K. K. Sørensen, P. A. McCourt, B. S. Ahluwalia and B. Smedsrød, Sci. Rep., 2020, 10, 1-9.

375 J. R. Newton-Northup, S. D. Figueroa, T. P. Quinn and S. L. Deutscher, Nucl. Med. Biol., 2009, 36, 789-800.

376 J. R. Newton, Y. Miao, S. L. Deutscher and T. P. Quinn, J. Nucl. Med., 2007, 48, 429-436.

377 M. Rusckowski, S. Gupta, G. Liu, S. Dou and D. J. Hnatowich, J. Nucl. Med., 2004, 45, 1201-1208.

378 M. Rusckowski, S. Gupta, G. Liu, S. Dou and D. J. Hnatowich, Nucl. Med. Biol., 2008, 35, 433-440.

379 M. Elena Cardoso, L. Fernández, E. Tejería, P. Esperón and M. Terán, Curr. Radiopharm., 2016, 9, 137-142.

380 D. Holman, M. P. Lungren, J. Hardy, C. Contag and F. Blankenberg, Bioconjugate Chem., 2017, 28, 2698-2706.

381 I. L. Aanei, A. M. Elsohly, M. E. Farkas, C. Netirojjanakul, M. Regan, S. Taylor Murphy, J. P. O'Neil, Y. Seo and M. B. Francis, Mol. Pharmaceutics, 2016, 13, 3764-3772.

382 M. E. Farkas, I. L. Aanei, C. R. Behrens, G. J. Tong, S. T. Murphy, J. P. O’Neil and M. B. Francis, Mol. Pharmaceutics, 2013, 10, 69-76.

383 Y. H. Chung, H. Cai and N. F. Steinmetz, Adv. Drug Delivery Rev., 2020, 156, 214-235, DOI: 10.1016/j.addr.2020.06.024.

384 M. Karimi, H. Mirshekari, S. M. Moosavi Basri, S. Bahrami, M. Moghoofei and M. R. Hamblin, Adv. Drug Delivery Rev., 2016, 106, 45-62.

385 C. Li and R. J. Samulski, Nat. Rev. Genet., 2020, 21, 255-272.

386 H. Cabral, K. Miyata, K. Osada and K. Kataoka, Chem. Rev., 2018, 118, 6844-6892.

387 M. Cagel, F. C. Tesan, E. Bernabeu, M. J. Salgueiro, M. B. Zubillaga, M. A. Moretton and D. A. Chiappetta, Eur. J. Pharm. Biopharm., 2017, 113, 211-228.

388 B. Hoang, H. Lee, R. M. Reilly and C. Allen, Mol. Pharmaceutics, 2009, 6, 581-592.

389 H. Fonge, H. Huang, D. Scollard, R. M. Reilly and C. Allen, J. Controlled Release, 2012, 157, 366-374.

390 R. Jain, S. Nabar, P. Dandekar, P. Hassan, V. Aswal, Y. Talmon, T. Shet, L. Borde, K. Ray and V. Patravale, Nanomedicine, 2010, 5, 575-587.

391 G. A. Abdelbary and M. I. Tadros, Int. J. Pharm., 2013, 452, 300-310.

392 S. A. Nour, N. S. Abdelmalak, M. J. Naguib, H. M. Rashed and A. B. Ibrahim, Drug Delivery, 2016, 23, 3681-3695.

393 F. Tesan, C. Cerqueira-Coutinho, J. Salgueiro, M. de Souza Albernaz, S. R. Pinto, S. R. Rezende Dos Reis, E. S. Bernardes, D. Chiapetta, M. Zubillaga and R. SantosOliveira, J. Drug Delivery Sci. Technol., 2016, 36, 95-98.

394 H. M. Rashed, R. N. Shamma and E. B. Basalious, Drug Delivery, 2017, 24, 181-187.

395 S. Dumoga, Y. Rai, A. N. Bhatt, A. K. Tiwari, S. Singh, A. K. Mishra and D. Kakkar, ACS Appl. Mater. Interfaces, 2017, 9, 22195-22211. 
396 F. C. Tesan, M. G. Portillo, M. A. Moretton, E. Bernabeu, D. A. Chiappetta, M. J. Salgueiro and M. B. Zubillaga, Nucl. Med. Biol., 2017, 44, 62-68.

397 E. Grotz, N. L. Tateosian, J. Salgueiro, E. Bernabeu, L. Gonzalez, M. L. Manca, N. Amiano, D. Valenti, M. Manconi, V. García, M. A. Moretton and D. A. Chiappetta, J. Drug Delivery Sci. Technol., 2019, 53, 101170.

398 F. C. Tesan, M. B. Nicoud, M. Nuñez, V. A. Medina, D. A. Chiappetta and M. J. Salgueiro, Contrast Media Mol. Imaging, 2019, 2019, 4087895.

399 E. Ribeiro, I. Alho, F. Marques, L. Gano, I. Correia, J. D. G. Correia, S. Casimiro, L. Costa, I. Santos and C. Fernandes, Int. J. Pharm., 2016, 515, 692-701.

400 C. M. R. Oda, R. S. Fernandes, S. C. de Araújo Lopes, M. C. de Oliveira, V. N. Cardoso, D. M. Santos, A. M. de Castro Pimenta, A. Malachias, R. Paniago, D. M. Townsend, P. M. Colletti, D. Rubello, R. J. Alves, A. L. B. de Barros and E. A. Leite, Biomed. Pharmacother., 2017, 89, 268-275.

401 C. M. R. Oda, A. L. B. de Barros, R. S. Fernandes, S. E. M. Miranda, M. X. Teixeira, V. N. Cardoso, M. C. Oliveira and E. A. Leite, J. Drug Delivery Sci. Technol., 2018, 46, 182-187.

402 M. Silindir-Gunay and A. Y. Ozer, J. Drug Delivery Sci. Technol., 2020, 56, 101571.

403 V. S. Trubetskoy, M. D. Frank-Kamenetsky, K. R. Whiteman, G. L. Wolf and V. P. Torchilin, Acad. Radiol., 1996, 3, 232-238.

404 Z. Yang, S. Zheng, W. J. Harrison, J. Harder, X. Wen, J. G. Gelovani, A. Qiao and C. Li, Biomacromolecules, 2007, 8, 3422-3428.

405 A. Accardo, D. Tesauro, L. Aloj, L. Tarallo, C. Arra, G. Mangiapia, M. Vaccaro, C. Pedone, L. Paduano and G. Morelli, ChemMedChem, 2008, 3, 594-602.

406 R. R. Patil, J. Yu, S. R. Banerjee, Y. Ren, D. Leong, X. Jiang, M. Pomper, B. Tsui, D. L. Kraitchman and H. Q. Mao, Mol. Ther., 2011, 19, 1626-1635.

407 R. Zhang, C. Xiong, M. Huang, M. Zhou, Q. Huang, X. Wen, D. Liang and C. Li, Biomaterials, 2011, 32, 5872-5879.

408 B. Hoang, R. M. Reilly and C. Allen, Biomacromolecules, 2012, 13, 455-465.

409 B. Hoang, S. N. Ekdawi, R. M. Reilly and C. Allen, Mol. Pharmaceutics, 2013, 10, 4229-4241.

410 Y. Hong, H. Zhu, J. Hu, X. Lin, F. Wang, C. Li and Z. Yang, Bioorg. Med. Chem. Lett., 2014, 24, 2781-2785.

411 A. Arranja, O. Ivashchenko, A. G. Denkova, K. Morawska, S. Van Vlierberghe, P. Dubruel, G. Waton, F. J. Beekman, F. Schosseler and E. Mendes, Mol. Pharmaceutics, 2016, 13, 1158-1165.

412 A. Arranja, A. G. Denkova, K. Morawska, G. Waton, S. Van Vlierberghe, P. Dubruel, F. Schosseler and E. Mendes, J. Controlled Release, 2016, 224, 126-135.

413 J. Sun, L. Sun, J. Li, J. Xu, Z. Wan, Z. Ouyang, L. Liang, S. Li and D. Zeng, Acta Biomater., 2018, 75, 312-322.

414 A. I. Jensen, T. Binderup, P. K. Ek, C. E. Grandjean, P. H. Rasmussen, A. Kjaer and T. L. Andresen, J. Labelled Compd. Radiopharm., 2017, 60, 366-374.
415 J. Guo, H. Hong, G. Chen, S. Shi, Q. Zheng, Y. Zhang, C. P. Theuer, T. E. Barnhart, W. Cai and S. Gong, Biomaterials, 2013, 34, 8323-8332.

416 Y. Xiao, H. Hong, A. Javadi, J. W. Engle, W. Xu, Y. Yang, Y. Zhang, T. E. Barnhart, W. Cai and S. Gong, Biomaterials, 2012, 33, 3071-3082.

417 A. C. Laan, C. Santini, L. Jennings, M. de Jong, M. R. Bernsen and A. G. Denkova, EJNMMI Res., 2016, 6, 12.

418 A. de la Fuente, S. Kramer, N. Mohr, S. Pektor, B. Klasen, N. Bausbacher, M. Miederer, R. Zentel and F. Rösch, Am. J. Nucl. Med. Mol. Imaging, 2019, 9, 67-83.

419 A. Prasannan, T. A. Debele, H. C. Tsai, C. C. Chao, C. P. Lin and G. H. Hsiue, RSC Adv., 2015, 5, 107455-107465.

420 Y. Zhang, M. Jeon, L. J. Rich, H. Hong, J. Geng, Y. Zhang, S. Shi, T. E. Barnhart, P. Alexandridis, J. D. Huizinga, M. Seshadri, W. Cai, C. Kim and J. F. Lovell, Nat. Nanotechnol., 2014, 9, 631-638.

421 P. Kesharwani, K. Jain and N. K. Jain, Prog. Polym. Sci., 2014, 39, 268-307.

422 W. G. Lesniak, S. Boinapally, S. R. Banerjee, B. Behnam Azad, C. A. Foss, C. Shen, A. Lisok, B. Wharram, S. Nimmagadda and M. G. Pomper, Mol. Pharmaceutics, 2019, 16, 2590-2604.

423 M. C. Parrott, S. R. Benhabbour, C. Saab, J. A. Lemon, S. Parker, J. F. Valliant and A. Adronov, J. Am. Chem. Soc., 2009, 131, 2906-2916.

424 D. Zhou, S. H. Kim, V. M. Carroll, C. S. Dence and J. A. Katzenellenbn, Org. Biomol. Chem., 2014, 12, 8696-8701.

425 K. Pant, D. Gröger, R. Bergmann, J. Pietzsch, J. Steinbach, B. Graham, L. Spiccia, F. Berthon, B. Czarny, L. Devel, V. Dive, H. Stephan and R. Haag, Bioconjugate Chem., 2015, 26, 906-918.

426 W. Ma, F. Fu, J. Zhu, R. Huang, Y. Zhu, Z. Liu, J. Wang, P. S. Conti, X. Shi and K. Chen, Nanoscale, 2018, 10, 6113-6124.

427 T. S. Humani, S. Sutari, T. Triningsih, M. Ramli, R. Ritawidya and R. D. Haryuni, ITB J. Math. Fundam. Sci., 2017, 49, 258-268.

428 L. Kovacs, M. Tassano, M. Cabrera, C. B. Zamboni, M. Fernández, R. M. Anjos and P. Cabral, Cancer Biother. Radiopharm., 2015, 30, 405-410.

429 B. Gibbens-Bandala, E. Morales-Avila, G. Ferro-Flores, C. Santos-Cuevas, M. Luna-Gutiérrez, G. Ramírez-Nava and B. Ocampo-García, Polymers, 2019, 11, 1572.

430 H. Mendoza-Nava, G. Ferro-Flores, F. de, M. Ramírez, B. Ocampo-García, C. Santos-Cuevas, L. Aranda-Lara, E. Azorín-Vega, E. Morales-Avila and K. Isaac-Olivé, J. Nanomater., 2016, 2016, 1-11.

431 A. Laznickova, V. Biricova, M. Laznicek and P. Hermann, Appl. Radiat. Isot., 2014, 84, 70-77.

432 C. Kojima, C. Regino, Y. Umeda, H. Kobayashi and K. Kono, Int. J. Pharm., 2010, 383, 293-296.

433 Y. Niki, M. Ogawa, R. Makiura, Y. Magata and C. Kojima, Nanomedicine, 2015, 11, 2119-2127.

434 H. Katsumi, M. Nishikawa, R. Hirosaki, T. Okuda, S. Kawakami, F. Yamashita, M. Hashida, T. Sakane and A. Yamamoto, Mol. Pharmaceutics, 2016, 13, 2867-2873. 
435 K. Sano, Y. Iwamiya, T. Kurosaki, M. Ogawa, Y. Magata, H. Sasaki, T. Ohshima, M. Maeda and T. Mukai, J. Controlled Release, 2014, 194, 310-315.

436 H. Kobayashi, N. Sato, T. Saga, Y. Nakamoto, T. Ishimori, S. Toyama, K. Togashi, J. Konishi and M. W. Brechbiel, Eur. J. Nucl. Med., 2000, 27, 1334-1339.

437 M. Mamede, T. Saga, H. Kobayashi, T. Ishimori, T. Higashi, N. Sato, M. W. Brechbiel and J. Konishi, Clin. Cancer Res., 2003, 9, 3756-3762.

438 H. Kobayashi, C. Wu, M. K. Kim, C. H. Paik, J. A. Carrasquillo and M. W. Brechbiel, Bioconjugate Chem., 1999, 10, 103-111.

439 A. Parat, D. Kryza, F. Degoul, J. Taleb, C. Viallard, M. Janier, A. Garofalo, P. Bonazza, L. Heinrich-Balard, R. Cohen, E. Miot-Noirault, J. M. Chezal, C. Billotey and D. Felder-Flesch, J. Mater. Chem. B, 2015, 3, 2560-2571.

440 V. Biricová, A. Lázničková, M. Lázníček, M. Polášek and P. Hermann, J. Pharm. Biomed. Anal., 2011, 56, 505-512.

441 H. Kobayashi, S. Kawamoto, T. Saga, N. Sato, T. Ishimori, J. Konishi, K. Ono, K. Togashi and M. W. Brechbiel, Bioconjugate Chem., 2001, 12, 587-593.

442 S. A. McNelles, S. D. Knight, N. Janzen, J. F. Valliant and A. Adronov, Biomacromolecules, 2015, 16, 3033-3041.

443 L. P. Sadowski, P. E. Edem, J. F. Valliant and A. Adronov, Macromol. Biosci., 2016, 16, 1475-1484.

444 M. Song, Z. Guo, M. Gao, C. Shi, D. Xu, L. You, X. Wu, X. Su, R. Zhuang, W. Pan, T. Liu and X. Zhang, Chem. Biol. Drug Des., 2017, 89, 755-761.

445 Z. Guo, L. You, C. Shi, M. Song, M. Gao, D. Xu, C. Peng, R. Zhuang, T. Liu, X. Su, J. Du and X. Zhang, Mol. Pharmaceutics, 2017, 14, 3780-3788.

446 Y. Zhang, Y. Sun, X. Xu, H. Zhu, L. Huang, X. Zhang, Y. Qi and Y. M. Shen, Bioorg. Med. Chem. Lett., 2010, 20, 927-931.

447 X. Xu, Y. Zhang, X. Wang, X. Guo, X. Zhang, Y. Qi and Y. M. Shen, Bioorg. Med. Chem., 2011, 19, 1643-1648.

448 Y. Zhang, Y. Sun, X. Xu, X. Zhang, H. Zhu, L. Huang, Y. Qi and Y. M. Shen, J. Med. Chem., 2010, 53, 3262-3272.

449 X. Chen, S. Dou, G. Liu, X. Liu, Y. Wang, L. Chen, M. Rusckowski and D. J. Hnatowich, Bioconjugate Chem., 2008, 19, 1518-1525.

450 M. Tassano, N. Oddone, M. Fernández, W. Porcal, M. F. García, W. Martínez-López, J. C. Benech and P. Cabral, Int. J. Radiat. Biol., 2018, 94, 664-670.

451 W. Cui, Y. Zhang, X. Xu and Y.-M. Shen, Med. Chem., 2012, 8, 727-731.

452 W. G. Lesniak, C. Chu, A. Jablonska, B. Behnam Azad, O. Zwaenepoel, M. Zawadzki, A. Lisok, M. G. Pomper, P. Walczak, J. Gettemans and M. Janowski, Eur. J. Nucl. Med. Mol. Imaging, 2019, 46, 1940-1951.

453 C. Wängler, S. Maschauer, O. Prante, M. Schäfer, R. Schirrmacher, P. Bartenstein, M. Eisenhut and B. Wängler, ChemBioChem, 2010, 11, 2168-2181.

454 A. Ghai, B. Singh, P. Panwar Hazari, M. K. Schultz, A. Parmar, P. Kumar, S. Sharma, D. Dhawan and A. Kumar Mishra, Appl. Radiat. Isot., 2015, 105, 40-46.
455 G. Fischer, B. Wängler and C. Wängler, Molecules, 2014, 19, 6952-6974.

456 S. Lindner, C. Michler, B. Wängler, P. Bartenstein, G. Fischer, R. Schirrmacher and C. Wängler, Bioconjugate Chem., 2014, 25, 489-500.

457 E. Orocio-Rodríguez, G. Ferro-Flores, C. L. Santos-Cuevas, F. de, M. Ramírez, B. E. Ocampo-García, E. Azorín-Vega and F. M. Sánchez-García, J. Nanosci. Nanotechnol., 2015, 15, 4159-4169.

458 S. M. Ghoreishi, A. Khalaj, A. Bitarafan-Rajabi, A. D. Azar, M. S. Ardestani and A. Assadi, Drug Res., 2017, 67, 149-155.

459 S. M. Ghoreishi, A. Khalaj, O. Sabzevari, L. Badrzadeh, P. Mohammadzadeh, S. S. Mousavi Motlagh, A. BitarafanRajabi and M. Shafiee Ardestani, Int. J. Nanomed., 2018, 13, 4671-4683.

460 M. S. Ardestani, A. Bitarafan-Rajabi, P. Mohammadzadeh, S. Mortazavi-Derazkola, O. Sabzevari, A. D. Azar, S. Kazemi, S. R. Hosseini and S. M. Ghoreishi, Bioorg. Chem., 2020, 96, 103572.

461 A. G. Khosroshahi, M. Amanlou, O. Sabzevari, F. J. Daha, M. R. Aghasadeghi, M. Ghorbani, M. S. Ardestani, M. S. Alavidjeh, S. M. Sadat, M. H. Pouriayevali, L. Mousavi and S. E. S. Ebrahimi, Curr. Med. Chem., 2012, 20, 123-133.

462 M. R. Tassano, P. F. Audicio, J. P. Gambini, M. Fernandez, J. P. Damian, M. Moreno, J. A. Chabalgoity, O. Alonso, J. C. Benech and P. Cabral, Bioorg. Med. Chem. Lett., 2011, 21, 5598-5601.

463 X. Xu, Y. Li, T. Cao, J. Cheng and Y. Zhang, J. Nanopart. Res., 2018, 20, 204.

464 D. S. Wilbur, P. M. Pathare, D. K. Hamlin, K. R. Buhler and R. L. Vessella, Bioconjugate Chem., 1998, 9, 813-825.

465 Y. Kurokawa, H. Sone, T.-T. Win-Shwe, Y. Zeng, H. Kimura, Y. Koyama, Y. Yagi, Y. Matsui, M. Yamazaki and S. Hirano, Int. J. Nanomed., 2017, 12, 3967-3975.

466 Y. Cheng, J. Zhu, L. Zhao, Z. Xiong, Y. Tang, C. Liu, L. Guo, W. Qiao, X. Shi and J. Zhao, Nanomedicine, 2016, 11, 1253-1266.

467 N. Song, L. Zhao, X. Xu, M. Zhu, C. Liu, N. Sun, J. Yang, X. Shi and J. Zhao, ACS Appl. Mater. Interfaces, 2020, 12, 12395-12406.

468 L. Zhao, J. Zhu, Y. Cheng, Z. Xiong, Y. Tang, L. Guo, X. Shi and J. Zhao, ACS Appl. Mater. Interfaces, 2015, 7, 19798-19808.

469 L. Trembleau, M. Simpson, R. W. Cheyne, I. Escofet, M. V. C. A. L. Appleyard, K. Murray, S. Sharp, A. M. Thompson and T. A. D. Smith, New J. Chem., 2011, 35, 2496-2502.

470 M. S. Cooper, M. T. Ma, K. Sunassee, K. P. Shaw, J. D. Williams, R. L. Paul, P. S. Donnelly and P. J. Blower, Bioconjugate Chem., 2012, 23, 1029-1039.

471 M. M. Herth, M. Barz, D. Moderegger, M. Allmeroth, M. Jahn, O. Thews, R. Zentel and F. Rösch, Biomacromolecules, 2009, 10, 1697-1703.

472 A. B. Benito, M. K. Aiertza, M. Marradi, L. Gil-Iceta, T. Shekhter Zahavi, B. Szczupak, M. Jiménez-González, T. Reese, E. Scanziani, L. Passoni, M. Matteoli, M. De 
Maglie, A. Orenstein, M. Oron-Herman, G. Kostenich, L. Buzhansky, E. Gazit, H.-J. Grande, V. Gómez-Vallejo, J. Llop and I. Loinaz, Biomacromolecules, 2016, 17, 3213-3221. 473 S. E. Dunn, A. G. A. Coombes, M. C. Garnett, S. S. Davis, M. C. Davies and L. Illum, J. Controlled Release, 1997, 44, 65-76.

474 M. Snehalatha, K. Venugopal, R. N. Saha, A. K. Babbar and R. K. Sharma, Drug Delivery, 2008, 15, 277-287.

475 K. K. Halder, B. Mandal, M. C. Debnath, H. Bera, L. K. Ghosh and B. K. Gupta, J. Drug Targeting, 2008, 16, 311-320. 476 N. Mondal, K. K. Halder, M. M. Kamila, M. C. Debnath, T. K. Pal, S. K. Ghosal, B. R. Sarkar and S. Ganguly, Int. J. Pharm., 2010, 397, 194-200.

477 H. Gupta, M. Aqil, R. K. Khar, A. Ali, A. Bhatnagar and G. Mittal, Nanomedicine, 2010, 6, 324-333.

478 K. Ramanlal Chaudhari, A. Kumar, V. K. Megraj Khandelwal, M. Ukawala, A. S. Manjappa, A. K. Mishra, J. Monkkonen and R. S. Ramachandra Murthy, J. Controlled Release, 2012, 158, 470-478.

479 D. Sharma, D. Maheshwari, G. Philip, R. Rana, S. Bhatia, M. Singh, R. Gabrani, S. K. Sharma, J. Ali, R. K. Sharma and S. Dang, BioMed Res. Int., 2014, 2014, 156010.

480 P. J. Das, P. Paul, B. Mukherjee, B. Mazumder, L. Mondal, R. Baishya, M. C. Debnath and K. S. Dey, Mol. Pharmaceutics, 2015, 12, 2651-2664.

481 R. Baishya, D. K. Nayak, D. Kumar, S. Sinha, A. Gupta, S. Ganguly and M. C. Debnath, Pharm. Res., 2016, 33, 2691-2703.

482 Z. He, X. Zhang, J. Huang, Y. Wu, X. Huang, J. Chen, J. Xia, H. Jiang, J. Ma and J. Wu, Oncotarget, 2016, 7, 76635-76646.

483 R. H. Gaonkar, S. Ganguly, S. Dewanjee, S. Sinha, A. Gupta, S. Ganguly, D. Chattopadhyay and M. Chatterjee Debnath, Sci. Rep., 2017, 7, 1-14.

484 I. Khan, A. Gothwal, A. Kaul, R. Mathur, A. K. Mishra and U. Gupta, Pharm. Res., 2018, 35, 1-11.

485 K. Nigam, A. Kaur, A. Tyagi, K. Manda, R. Gabrani and S. Dang, Rejuvenation Res., 2019, 22, 235-245.

486 L. Mondal, B. Mukherjee, K. Das, S. Bhattacharya, D. Dutta, S. Chakraborty, M. M. Pal, R. H. Gaonkar and M. C. Debnath, Int. J. Nanomed., 2019, 14, 8073-8094.

487 R. W. Sirianni, M. Q. Zheng, T. R. Patel, T. Shafbauer, J. Zhou, W. M. Saltzman, R. E. Carson and Y. Huang, Bioconjugate Chem., 2014, 25, 2157-2165.

488 S. Subramanian, U. Pandey, D. Gugulothu, V. Patravale and G. Samuel, Cancer Biother. Radiopharm., 2013, 28, 598-606.

489 M. R. Gill, J. U. Menon, P. J. Jarman, J. Owen, I. SkaripaKoukelli, S. Able, J. A. Thomas, R. Carlisle and K. A. Vallis, Nanoscale, 2018, 10, 10596-10608.

490 R. M. Trujillo-Nolasco, E. Morales-Avila, B. E. OcampoGarcía, G. Ferro-Flores, B. V. Gibbens-Bandala, A. EscuderoCastellanos and K. Isaac-Olive, Mater. Sci. Eng., C, 2019, 103, 109766.

491 B. Gibbens-Bandala, E. Morales-Avila, G. Ferro-Flores, C. Santos-Cuevas, L. Meléndez-Alafort, M. TrujilloNolasco and B. Ocampo-García, Mater. Sci. Eng., C, 2019, 105, 110043.
492 G. Arora, J. Shukla, S. Ghosh, S. K. Maulik, A. Malhotra and G. Bandopadhyaya, PLoS One, 2012, 7, e34019.

493 M. Allmeroth, D. Moderegger, B. Biesalski, K. Koynov, F. Rösch, O. Thews and R. Zentel, Biomacromolecules, 2011, 12, 2841-2849.

494 M. Allmeroth, D. Moderegger, D. Gündel, K. Koynov, H. G. Buchholz, K. Mohr, F. Rösch, R. Zentel and O. Thews, Biomacromolecules, 2013, 14, 3091-3101.

495 M. Allmeroth, D. Moderegger, D. Gündel, H. G. Buchholz, N. Mohr, K. Koynov, F. Rösch, O. Thews and R. Zentel, J. Controlled Release, 2013, 172, 77-85.

496 M. M. Herth, M. Barz, M. Jahn, R. Zentel and F. Rösch, Bioorg. Med. Chem. Lett., 2010, 20, 5454-5458.

497 M. C. Chen, H. S. Wong, K. J. Lin, H. L. Chen, S. P. Wey, K. Sonaje, Y. H. Lin, C. Y. Chu and H. W. Sung, Biomaterials, 2009, 30, 6629-6637.

498 A. Polyák, I. Hajdu, M. Bodnár, G. Trencsényi, Z. Pöstényi, V. Haász, G. Jánoki, G. A. Jánoki, L. Balogh and J. Borbély, Int. J. Pharm., 2013, 449, 10-17.

499 A. Polyák, I. Hajdu, M. Bodnár, G. Dabasi, R. P. Jóba, J. Borbély and L. Balogh, Int. J. Pharm., 2014, 474, 91-94.

500 H. Rajabi, S. Rasaneh and S. Salehi, Synth. React. Inorg., Met.-Org., Nano-Met. Chem., 2016, 46, 1450-1454.

501 I. S. Ahmed, H. M. Rashed, H. Fayez, F. Farouk and R. N. Shamma, Pharmaceutics, 2020, 12, 107.

502 J. R. Franca, L. L. Fuscaldi, T. G. Ribeiro, G. Foureaux, A. L. A. Cesar, R. O. Castilho, S. Cronemberger, A. J. Ferreira, S. O. A. Fernandes, V. N. Cardoso and A. A. G. Faraco, J. Biomed. Mater. Res., Part B, 2020, 108, 2227-2237.

503 M. Fairclough, C. Prenant, B. Ellis, H. Boutin, A. McMahon, G. Brown, P. Locatelli and A. K. P. Jones, J. Labelled Compd. Radiopharm., 2016, 59, 270-276.

504 M. Fairclough, B. Ellis, H. Boutin, A. K. P. Jones, A. McMahon, S. Alzabin, A. Gennari and C. Prenant, Appl. Radiat. Isot., 2017, 130, 7-12.

505 D. E. Lee, J. H. Na, S. Lee, C. M. Kang, H. N. Kim, S. J. Han, H. Kim, Y. S. Choe, K. H. Jung, K. C. Lee, K. Choi, I. C. Kwon, S. Y. Jeong, K. H. Lee and K. Kim, Mol. Pharmaceutics, 2013, 10, 2190-2198.

506 S. Lee, S. W. Kang, J. H. Ryu, J. H. Na, D. E. Lee, S. J. Han, C. M. Kang, Y. S. Choe, K. C. Lee, J. F. Leary, K. Choi, K. H. Lee and K. Kim, Bioconjugate Chem., 2014, 25, 601-610.

507 Z. Körhegyi, D. Rózsa, I. Hajdu, M. Bodnár, I. Kertész, K. Kerekes, S. Kun, J. Kollár, J. Varga, I. Garai, G. Trencsényi and J. Borbély, Anticancer Res., 2019, 39, 2415-2427.

508 E. J. Keliher, Y.-X. Ye, G. R. Wojtkiewicz, A. D. Aguirre, B. Tricot, M. L. Senders, H. Groenen, F. Fay, C. PerezMedina, C. Calcagno, G. Carlucci, T. Reiner, Y. Sun, G. Courties, Y. Iwamoto, H.-Y. Kim, C. Wang, J. W. Chen, F. K. Swirski, H.-Y. Wey, J. Hooker, Z. A. Fayad, W. J. M. Mulder, R. Weissleder and M. Nahrendorf, Nat. Commun., 2017, 8, 14064.

509 R. Gracia, M. Marradi, U. Cossío, A. Benito, A. Pérez-San Vicente, V. Gómez-Vallejo, H. J. Grande, J. Llop and I. Loinaz, J. Mater. Chem. B, 2017, 5, 1143-1147. 
510 M. D. Majmudar, J. Yoo, E. J. Keliher, J. J. Truelove, Y. Iwamoto, B. Sena, P. Dutta, A. Borodovsky, K. Fitzgerald, M. F. Di Carli, P. Libby, D. G. Anderson, F. K. Swirski, R. Weissleder and M. Nahrendorf, Circ. Res., 2013, 112, 755-761.

511 R. Rossin, D. Pan, K. Qi, J. L. Turner, X. Sun, K. L. Wooley and M. J. Welch, J. Nucl. Med., 2005, 46, 1210-1218.

512 J. Xu, G. Sun, R. Rossin, A. Hagooly, Z. Li, K. I. Fukukawa, B. W. Messmore, D. A. Moore, M. J. Welch, C. J. Hawker and K. L. Wooley, Macromolecules, 2007, 40, 2971-2973.

513 G. Sun, J. Xu, A. Hagooly, R. Rossin, Z. Li, D. A. Moore, C. J. Hawker, M. J. Welch and K. L. Wooley, Adv. Mater., 2007, 19, 3157-3162.

514 G. Sun, A. Hagooly, J. Xu, A. M. Nyström, Z. Li, R. Rossin, D. A. Moore, K. L. Wooley and M. J. Welch, Biomacromolecules, 2008, 9, 1997-2006.

515 A. Li, H. P. Luehmann, G. Sun, S. Samarajeewa, J. Zou, S. Zhang, F. Zhang, M. J. Welch, Y. Liu and K. L. Wooley, ACS Nano, 2012, 6, 8970-8982.

516 T. J. Beldman, M. L. Senders, A. Alaarg, C. Pérez-Medina, J. Tang, Y. Zhao, F. Fay, J. Deichmöller, B. Born, E. Desclos, N. N. Van Der Wel, R. A. Hoebe, F. Kohen, E. Kartvelishvily, M. Neeman, T. Reiner, C. Calcagno, Z. A. Fayad, M. P. J. De Winther, E. Lutgens, W. J. M. Mulder and E. Kluza, ACS Nano, 2017, 11, 5785-5799.

517 D. R. Beckford Vera, S. D. Fontaine, H. F. VanBrocklin, B. R. Hearn, R. Reid, G. W. Ashley and D. V. Santi, Mol. Cancer Ther., 2020, 19, 673-679.

518 S. Soni, A. K. Babbar, R. K. Sharma and A. Maitra, J. Drug Targeting, 2006, 14, 87-95.

519 S. Singh, B. Bingöl, A. Morgenroth, F. M. Mottaghy, M. Möller and J. Schmaljohann, Macromol. Rapid Commun., 2013, 34, 562-567.

520 N. Drude, S. Singh, O. H. Winz, M. Möller, F. M. Mottaghy and A. Morgenroth, Biomacromolecules, 2017, 18, 2489-2498.

521 S. Berke, A. L. Kampmann, M. Wuest, J. J. Bailey, B. Glowacki, F. Wuest, K. Jurkschat, R. Weberskirch and R. Schirrmacher, Bioconjugate Chem., 2018, 29, 89-95.

522 K. Sano, Y. Kanada, K. Takahashi, N. Ding, K. Kanazaki, T. Mukai, M. Ono and H. Saji, Mol. Pharmaceutics, 2018, 15, 3997-4003.

523 K. Sano, Y. Kanada, K. Kanazaki, N. Ding, M. Ono and H. Saji, J. Nucl. Med., 2017, 58, 1380-1385.

524 S. R. R. dos Reis, S. R. Pinto, F. D. de Menezes, R. MartinezManez, E. Ricci-Junior, L. M. R. Alencar, E. Helal-Neto, A. O. da Silva de Barros, P. C. Lisboa and R. SantosOliveira, Pharm. Res., 2020, 37, 1-12.

525 D. Shenoy, S. Little, R. Langer and M. Amiji, Pharm. Res., 2005, 22, 2107-2114.

526 P. K. Woodard, Y. Liu, E. D. Pressly, H. P. Luehmann, L. Detering, D. E. Sultan, R. Laforest, A. J. McGrath, R. J. Gropler and C. J. Hawker, Pharm. Res., 2016, 33, 2400-2410.

527 Y. Liu, H. P. Luehmann, L. Detering, E. D. Pressly, A. J. McGrath, D. Sultan, A. Nguyen, S. Grathwohl, M. Shokeen, M. Zayed, R. J. Gropler, D. Abendschein,
C. J. Hawker and P. K. Woodard, ACS Appl. Mater. Interfaces, 2019, 11, 15316-15321.

528 R. Abellan-Pose, M. Rodríguez-Évora, S. Vicente, N. Csaba, C. Évora, M. J. Alonso and A. Delgado, Eur. J. Pharm. Biopharm., 2017, 112, 155-163.

529 P. P. Di Mauro, V. Gómez-Vallejo, Z. Baz Maldonado, J. Llop Roig and S. Borrós, Bioconjugate Chem., 2015, 26, 582-592.

530 B. Jia, X. Zhang, B. Wang, M. Chen, F. Lv, S. Wang and F. Wang, ACS Appl. Mater. Interfaces, 2018, 10, 6646-6651.

531 S. Nascimento dos Santos, S. Rhaissa Rezende dos Reis, S. Rocha Pinto, C. Cerqueira-Coutinho, F. Nigro, T. Christina Barja-Fidalgo, N. Martins Alexandre Pinheiro, H. Affonso Paula Neto and R. Santos-Oliveira, J. Nanopart. Res., 2017, 19, 345, DOI: 10.1007/s11051-017-4037-x.

532 T. L. Braga, S. R. Pinto, S. R. R. dos Reis, F. L. Portilho, A. O. da Silva de Barros, E. S. Bernardes, S. N. dos Santos, L. M. R. Alencar, E. Ricci-Junior and R. Santos-Oliveira, Pharm. Res., 2019, 36, 1-8.

533 M. A. Pereira, V. C. F. Mosqueira, J. M. C. Vilela, M. S. Andrade, G. A. Ramaldes and V. N. Cardoso, Eur. J. Pharm. Sci., 2008, 33, 42-51.

534 M. A. Pereira, V. C. F. Mosqueira, V. A. S. Carmo, C. S. Ferrari, E. C. O. Reis, G. A. Ramaldes and V. N. Cardoso, Nucl. Med. Commun., 2009, 30, 749-755.

535 G. Wunderlich, T. Grüning, B. R. Paulke, A. Lieske and J. Kotzerke, Nucl. Med. Biol., 2004, 31, 87-92.

536 P. Areses, M. T. Agüeros, G. Quincoces, M. Collantes, J. Á. Richter, L. M. López-Sánchez, M. Sánchez-Martínez, J. M. Irache and I. Penuelas, Mol. Imaging Biol., 2011, 13, 1215-1223.

537 G. Kaul and M. Amiji, J. Drug Targeting, 2004, 12, 585-591.

538 S. Kommareddy and M. Amiji, J. Pharm. Sci., 2007, 96, 397-407.

539 M. D. Bhavsar and M. M. Amiji, AAPS PharmSciTech, 2008, 9, 288-294.

540 J. Xu, F. Gattacceca and M. Amiji, Mol. Pharmaceutics, 2013, 10, 2031-2044.

541 J. A. C. M. Goos, A. Cho, L. M. Carter, T. R. Dilling, M. Davydova, K. Mandleywala, S. Puttick, A. Gupta, W. S. Price, J. F. Quinn, M. R. Whittaker, J. S. Lewis and T. P. Davis, Theranostics, 2020, 10, 567-584.

542 K. R. Chaudhari, M. Ukawala, A. S. Manjappa, A. Kumar, P. K. Mundada, A. K. Mishra, R. Mathur, J. Mönkkönen and R. S. Ramchandra Murthy, Pharm. Res., 2012, 29, 53-68.

543 Q. Fan, K. Cheng, X. Hu, X. Ma, R. Zhang, M. Yang, X. Lu, L. Xing, W. Huang, S. S. Gambhir and Z. Cheng, J. Am. Chem. Soc., 2014, 136, 15185-15194.

544 H. Zhou, Q. Zhang, Y. Cheng, L. Xiang, G. Shen, X. Wu, H. Cai, D. Li, H. Zhu, R. Zhang, L. Li and Z. Cheng, Nanomedicine, 2020, 29, 102248.

545 T. Martínez Martínez, Á. García Aliaga, I. López-González, A. Abella Tarazona, M. J. Ibáñez Ibáñez, J. L. Cenis, L. Meseguer-Olmo and A. A. Lozano-Pérez, ACS Biomater. Sci. Eng., 2020, 6, 3299-3309. 
546 G. Shim, M. G. Kim, J. Y. Park and Y. K. Oh, Adv. Drug Delivery Rev., 2016, 105, 205-227.

547 B. S. Wong, S. L. Yoong, A. Jagusiak, T. Panczyk, H. K. Ho, W. H. Ang and G. Pastorin, Adv. Drug Delivery Rev., 2013, 65, 1964-2015.

548 H. Gong, R. Peng and Z. Liu, Adv. Drug Delivery Rev., 2013, 65, 1951-1963.

549 J. Lin, X. Chen and P. Huang, Adv. Drug Delivery Rev., 2016, 105, 242-254.

550 S. Shi, C. Xu, K. Yang, S. Goel, H. F. Valdovinos, H. Luo, E. B. Ehlerding, C. G. England, L. Cheng, F. Chen, R. J. Nickles, Z. Liu and W. Cai, Angew. Chem., Int. Ed., 2017, 56, 2889-2892.

551 S. Sarpaki, F. Cortezon-Tamarit, S. R. M. M. de Aguiar, R. M. Exner, D. Divall, R. L. Arrowsmith, H. Ge, F. J. Palomares, L. Carroll, D. G. Calatayud, S. J. Paisey, E. O. Aboagye and S. I. Pascu, Nanoscale, 2020, 12, 6603-6608.

552 K. Yang, J. Wan, S. Zhang, Y. Zhang, S. T. Lee and Z. Liu, ACS Nano, 2011, 5, 516-522.

553 L. Zhan, G. Yanxia, Z. Xiaoyong, Q. Wei, F. Qiaohui, L. Yan, J. Zongxian, W. Jianjun, T. Yuqin, D. Xiaojiang and W. Wangsuo, J. Nanopart. Res., 2011, 13, 2939-2947.

554 Q. Wei, L. Zhan, B. Juanjuan, W. Jing, W. Jianjun, S. Taoli, G. Yi'an and W. Wangsuo, Nanoscale Res. Lett., 2012, 7, 473.

555 S. B. Challan and A. Massoud, J. Radioanal. Nucl. Chem., 2017, 314, 2189-2199.

556 F. Yurt, O. A. Ersöz, E. Harputlu and K. Ocakoglu, Chem. Biol. Drug Des., 2018, 91, 1094-1100.

557 M. Das, S. R. Datir, R. P. Singh and S. Jain, Mol. Pharmaceutics, 2013, 10, 2543-2557.

558 R. S. Fernandes, J. A. Lemos, A. L. Branco de Barros, V. Geraldo, E. Eleto da Silva, L. Alisaraie and D. C. Ferreira Soares, J. Drug Delivery Sci. Technol., 2019, 50, 266-277.

559 T. Cao, X. Zhou, Y. Zheng, Y. Sun, J. Zhang, W. Chen, J. Zhang, Z. Zhou, S. Yang, Y. Zhang, H. Yang and M. Wang, ACS Appl. Mater. Interfaces, 2017, 9, 42612-42621.

560 X. Zhang, J. Yin, C. Kang, J. Li, Y. Zhu, W. Li, Q. Huang and Z. Zhu, Toxicol. Lett., 2010, 198, 237-243.

561 X. Zhang, J. Yin, C. Peng, W. Hu, Z. Zhu, W. Li, C. Fan and Q. Huang, Carbon, 2011, 49, 986-995.

562 X. Zhang, Y. Zhu, J. Li, Z. Zhu, J. Li, W. Li and Q. Huang, J. Nanopart. Res., 2011, 13, 6941-6952.

563 M. R. McDevitt, D. Chattopadhyay, B. J. Kappel, J. S. Jaggi, S. R. Schiffman, C. Antczak, J. T. Njardarson, R. Brentjens and D. A. Scheinberg, J. Nucl. Med., 2007, 48, 1180-1189.

564 M. R. McDevitt, D. Chattopadhyay, J. S. Jaggi, R. D. Finn, P. B. Zanzonico, C. Villa, D. Rey, J. Mendenhall, C. A. Batt, J. T. Njardarson and D. A. Scheinberg, PLoS One, 2007, 2, e907.

565 C. H. Villa, M. R. McDevitt, F. E. Escorcia, D. A. Rey, M. Bergkvist, C. A. Batt and D. A. Scheinberg, Nano Lett., 2008, 8, 4221-4228.

566 L. Lacerda, A. Soundararajan, R. Singh, G. Pastorin, K. T. Al-Jamal, J. Turton, P. Frederik, M. A. Herrero, S. Li, A. Bao, D. Emfietzoglou, S. Mather, W. T. Phillips, M. Prato,
A. Bianco, B. Goins and K. Kostarelos, Adv. Mater., 2008, 20, 225-230.

567 M. A. Herrero, L. Lacerda, A. Bianco, K. Kostarelos and M. Prato, Int. J. Nanotechnol., 2011, 8, 885-897.

568 B. Cornelissen, S. Able, V. Kersemans, P. A. Waghorn, S. Myhra, K. Jurkshat, A. Crossley and K. A. Vallis, Biomaterials, 2013, 34, 1146-1154.

569 H. Kafa, J. T. W. Wang, N. Rubio, K. Venner, G. Anderson, E. Pach, B. Ballesteros, J. E. Preston, N. J. Abbott and K. T. Al-Jamal, Biomaterials, 2015, 53, 437-452.

570 M. Zhang, D. Jasim, C. Ménard-Moyon, A. Nunes, S. Iijima, A. Bianco, M. Yudasaka and K. Kostarelos, Int. J. Nanomed., 2016, 11, 3317-3330.

571 H. Kafa, J. T. W. Wang, N. Rubio, R. Klippstein, P. M. Costa, H. A. F. M. Hassan, J. K. Sosabowski, S. S. Bansal, J. E. Preston, N. J. Abbott and K. T. Al-Jamal, J. Controlled Release, 2016, 225, 217-229.

572 J. T. W. Wang, N. Rubio, H. Kafa, E. Venturelli, C. Fabbro, C. Ménard-Moyon, T. Da Ros, J. K. Sosabowski, A. D. Lawson, M. K. Robinson, M. Prato, A. Bianco, F. Festy, J. E. Preston, K. Kostarelos and K. T. Al-Jamal, J. Controlled Release, 2016, 224, 22-32.

573 J. Da-wei, P. Cheng, S. Yan-Hong, J. Li-Na, L. Jian-Bo and Z. Lan, Nucl. Sci. Tech., 2015, 26(4), 040301, DOI: 10.13538/ j.1001-8042/nst.26.040301.

574 P. Rong, K. Yang, A. Srivastan, D. O. Kiesewetter, X. Yue, F. Wang, L. Nie, A. Bhirde, Z. Wang, Z. Liu, G. Niu, W. Wang and X. Chen, Theranostics, 2014, 4, 229-239.

575 Z. Liu, W. Cai, L. He, N. Nakayama, K. Chen, X. Sun, X. Chen and H. Dai, Nat. Nanotechnol., 2007, 2, 47-52.

576 Z. Liu, S. M. Tabakman, Z. Chen and H. Dai, Nat. Protoc., 2009, 4, 1372-1382.

577 G. Biagiotti, F. Pisaneschi, S. T. Gammon, F. Machetti, M. C. Ligi, G. Giambastiani, G. Tuci, E. Powell, H. PiwnicaWorms, E. Pranzini, P. Paoli, S. Cicchi and D. PiwnicaWorms, J. Mater. Chem. B, 2019, 7, 2678-2687.

578 H. Hong, K. Yang, Y. Zhang, J. W. Engle, L. Feng, Y. Yang, T. R. Nayak, S. Goel, J. Bean, C. P. Theuer, T. E. Barnhart, Z. Liu and W. Cai, ACS Nano, 2012, 6, 2361-2370.

579 D. Yang, L. Feng, C. A. Dougherty, K. E. Luker, D. Chen, M. A. Cauble, M. M. Banaszak Holl, G. D. Luker, B. D. Ross, Z. Liu and H. Hong, Biomaterials, 2016, 104, 361-371.

580 J. Li, W. Yang, R. Cui, D. Wang, Y. Chang, W. Gu, W. Yin, X. Bai, K. Chen, L. Xia, H. Geng and G. Xing, Nanotechnology, 2016, 27, 155101.

581 H. Hong, Y. Zhang, J. W. Engle, T. R. Nayak, C. P. Theuer, R. J. Nickles, T. E. Barnhart and W. Cai, Biomaterials, 2012, 33, 4147-4156.

582 J. Lamb, E. Fischer, M. Rosillo-Lopez, C. G. Salzmann and J. P. Holland, Chem. Sci., 2019, 10, 8880-8888.

583 M. McDevitt, M. McDevitt, A. Ruggiero, C. H. Villa, J. P. Holland, S. R. Sprinkle, C. May, J. Lewis and D. Scheinberg, Int. J. Nanomed., 2010, 5, 783.

584 R. W. Ormsby, T. McNally, C. A. Mitchell, A. Musumeci, T. Schiller, P. Halley, L. Gahan, D. Martin, S. V. Smith and N. J. Dunne, Acta Mater., 2014, 64, 54-61. 
585 D. Satpati, A. Satpati, Y. Pamale, C. Kumar, R. Sharma, H. D. Sarma and S. Banerjee, RSC Adv., 2016, 6, 50761-50769.

586 S. Liu, Chem. Soc. Rev., 2004, 33, 445-461.

587 B. R. Jarrett, B. Gustafsson, D. L. Kukis and A. Y. Louie, Bioconjugate Chem., 2008, 19, 1496-1504.

588 H.-Y. Lee, Z. Li, K. Chen, A. R. Hsu, C. Xu, J. Xie, S. Sun and X. Chen, J. Nucl. Med., 2008, 49, 1371-1379.

589 M. Yang, K. Cheng, S. Qi, H. Liu, Y. Jiang, H. Jiang, J. Li, K. Chen, H. Zhang and Z. Cheng, Biomaterials, 2013, 34, 2796-2806.

590 C. G. England, H.-J. Im, L. Feng, F. Chen, S. A. Graves, R. Hernandez, H. Orbay, C. Xu, S. Y. Cho, R. J. Nickles, Z. Liu, D. S. Lee and W. Cai, Biomaterials, 2016, 100, 101-109.

591 J. Zhang, Y. Ma, W. Yang, J. Xue, Y. Ding, C. Xie, W. Luo, F. Gao, Z. Zhang, Y. Zhao, Z. Chai and X. He, Nanoscale, 2019, 11, 5909-5913.

592 X. Cui, S. Belo, D. Krüger, Y. Yan, R. T. M. de Rosales, M. Jauregui-Osoro, H. Ye, S. Su, D. Mathe, N. Kovács, I. Horváth, M. Semjeni, K. Sunassee, K. Szigeti, M. A. Green and P. J. Blower, Biomaterials, 2014, 35, 5840-5846.

593 N. Gholipour, M. Akhlaghi, A. Mokhtari Kheirabadi, P. Geramifar and D. Beiki, Int. J. Biol. Macromol., 2020, 148, 932-941.

594 A. Lahooti, S. Shanehsazzadeh and S. Laurent, Nanotechnology, 2020, 31, 015102.

595 M. Hajiramezanali, F. Atyabi, M. Mosayebnia, M. Akhlaghi, P. Geramifar, A. R. Jalilian, S. M. Mazidi, H. Yousefnia, S. Shahhosseini and D. Beiki, Int. J. Nanomed., 2019, 14, 2591-2605.

596 S. Kim, M. K. Chae, M. S. Yim, I. H. Jeong, J. Cho, C. Lee and E. K. Ryu, Biomaterials, 2013, 34, 8114-8121.

597 P. Unak, V. Tekin, O. K. Guldu and O. Aras, Appl. Organomet. Chem., 2020, 34, e5616, DOI: 10.1002/aoc.5616.

598 Z. Sun, K. Cheng, F. Wu, H. Liu, X. Ma, X. Su, Y. Liu, L. Xia and Z. Cheng, Nanoscale, 2016, 8, 19644-19653.

599 M. Ognjanović, M. Radović, M. Mirković, Ž. Prijović, M. del Puerto Morales, M. Čeh, S. Vranješ-Đurić and B. Antić, ACS Appl. Mater. Interfaces, 2019, 11, 41109-41117.

600 R. Torres Martin de Rosales, R. Tavaré, A. Glaria, G. Varma, A. Protti and P. J. Blower, Bioconjugate Chem., 2011, 22, 455-465.

601 L. Sandiford, A. Phinikaridou, A. Protti, L. K. Meszaros, X. Cui, Y. Yan, G. Frodsham, P. A. Williamson, N. Gaddum, R. M. Botnar, P. J. Blower, M. A. Green and R. T. M. de Rosales, ACS Nano, 2013, 7, 500-512.

602 A. Adamiano, M. Iafisco, M. Sandri, M. Basini, P. Arosio, T. Canu, G. Sitia, A. Esposito, V. Iannotti, G. Ausanio, E. Fragogeorgi, M. Rouchota, G. Loudos, A. Lascialfari and A. Tampieri, Acta Biomater., 2018, 73, 458-469.

603 M. R. A. Abdollah, T. J. Carter, C. Jones, T. L. Kalber, V. Rajkumar, B. Tolner, C. Gruettner, M. Zaw-Thin, J. Baguña Torres, M. Ellis, M. Robson, R. B. Pedley, P. Mulholland, R. T. M. de Rosales and K. A. Chester, ACS Nano, 2018, 12, 1156-1169.

604 C. Tsoukalas, D. Psimadas, G. A. Kastis, V. Koutoulidis, A. L. Harris, M. Paravatou-Petsotas, M. Karageorgou,
L. R. Furenlid, L. A. Moulopoulos, D. Stamopoulos and P. Bouziotis, Front. Chem., 2018, 6, 224, DOI: 10.3389/ fchem.2018.00224.

605 S. Fatahian, D. Shahbazi-Gahrouei, M. Pouladian, M. H. Yousefi, G. R. Amiri and A. Noori, J. Radioanal. Nucl. Chem., 2012, 293, 915-921.

606 M. Felber and R. Alberto, Nanoscale, 2015, 7, 6653-6660.

607 M. de Souza Albernaz, S. H. Toma, J. Clanton, K. Araki and R. Santos-Oliveira, Pharm. Res., 2018, 35, 24.

608 Y. Zhao, Q. Yao, H. Tan, B. Wu, P. Hu, P. Wu, Y. Gu, C. Zhang, D. Cheng and H. Shi, J. Radioanal. Nucl. Chem., 2014, 299, 1273-1280.

609 C.-M. Lee, H.-J. Jeong, E.-M. Kim, D. W. Kim, S. T. Lim, H. T. Kim, I.-K. Park, Y. Y. Jeong, J. W. Kim and M.H. Sohn, Magn. Reson. Med., 2009, 62, 1440-1446.

610 M. Zaw Thin, H. Allan, R. Bofinger, T. D. Kostelec, S. Guillaume, J. J. Connell, P. S. Patrick, H. C. Hailes, A. B. Tabor, M. F. Lythgoe, D. J. Stuckey and T. L. Kalber, Nanoscale, 2020, 12, 16570-16585, DOI: 10.1039/D0NR03237A.

611 M. Radović, M. Mirković, M. Perić, D. Janković, A. Vukadinović, D. Stanković, Đ. Petrović, M. Bošković, B. Antić, M. Marković and S. Vranješ-Đurić, J. Mater. Chem. $B, 2017,5,8738-8747$.

612 M. Radović, M. P. Calatayud, G. F. Goya, M. R. Ibarra, B. Antić, V. Spasojević, N. Nikolić, D. Janković, M. Mirković and S. Vranješ-Đurić, J. Biomed. Mater. Res., Part A, 2015, 103, 126-134.

613 B. Azadbakht, H. Afarideh, M. Ghannadi-Maragheh, A. Bahrami-Samani and M. Asgari, Nucl. Med. Biol., 2017, 48, 26-30.

614 J. Cao, Y. Wang, J. Yu, J. Xia, C. Zhang, D. Yin and U. O. Häfeli, J. Magn. Magn. Mater., 2004, 277, 165-174.

615 S. Akbari-Karadeh, S. M. R. Aghamiri, P. TajerMohammad-Ghazvini and S. Ghorbanzadeh-Mashkani, Appl. Biochem. Biotechnol., 2020, 190, 540-550.

616 Y. H. Gholami, L. Josephson, E. A. Akam, P. Caravan, M. Q. Wilks, X.-Z. Pan, R. Maschmeyer, A. Kolnick, G. El Fakhri, M. D. Normandin, Z. Kuncic and H. Yuan, Int. J. Nanomed., 2020, 15, 31-47.

617 A. Ruiz-de-Angulo, A. Zabaleta, V. Gómez-Vallejo, J. Llop and J. C. Mareque-Rivas, ACS Nano, 2016, 10, 1602-1618.

618 P. S. Patrick, L. K. Bogart, T. J. Macdonald, P. Southern, M. J. Powell, M. Zaw-Thin, N. H. Voelcker, I. P. Parkin, Q. A. Pankhurst, M. F. Lythgoe, T. L. Kalber and J. C. Bear, Chem. Sci., 2019, 10, 2592-2597.

619 M. D. Normandin, H. Yuan, M. Q. Wilks, H. H. Chen, J. M. Kinsella, H. Cho, N. J. Guehl, N. Absi-Halabi, S. M. Hosseini, G. El Fakhri, D. E. Sosnovik and L. Josephson, Angew. Chem., Int. Ed., 2015, 54, 13002-13006.

620 R. Chakravarty, H. F. Valdovinos, F. Chen, C. M. Lewis, P. A. Ellison, H. Luo, M. E. Meyerand, R. J. Nickles and W. Cai, Adv. Mater., 2014, 26, 5119-5123.

621 B. Freund, U. I. Tromsdorf, O. T. Bruns, M. Heine, A. Giemsa, A. Bartelt, S. C. Salmen, N. Raabe, J. Heeren, H. Ittrich, R. Reimer, H. Hohenberg, U. Schumacher, H. Weller and P. Nielsen, ACS Nano, 2012, 6, 7318-7325. 
622 R. Madru, P. Kjellman, F. Olsson, K. Wingardh, C. Ingvar, F. Stahlberg, J. Olsrud, J. Latt, S. Fredriksson, L. Knutsson and S.-E. Strand, J. Nucl. Med., 2012, 53, 459-463.

623 I. Tsiapa, E. K. Efthimiadou, E. Fragogeorgi, G. Loudos, A. D. Varvarigou, P. Bouziotis, G. C. Kordas, D. Mihailidis, G. C. Nikiforidis, S. Xanthopoulos, D. Psimadas, M. Paravatou-Petsotas, L. Palamaris, J. D. Hazle and G. C. Kagadis, J. Colloid Interface Sci., 2014, 433, 163-175. 624 O. Mokhodoeva, M. Vlk, E. Málková, E. Kukleva, P. Mičolová, K. Štamberg, M. Šlouf, R. Dzhenloda and J. Kozempel, J. Nanopart. Res., 2016, 18, 301.

625 J. Pellico, J. Llop, I. Fernández-Barahona, R. Bhavesh, J. Ruiz-Cabello and F. Herranz, Contrast Media Mol. Imaging, 2017, 2017, 1-24.

626 F. Ai, C. A. Ferreira, F. Chen and W. Cai, Wiley Interdiscip. Rev.: Nanomed. Nanobiotechnol., 2016, 8, 619-630.

627 F. Ai, C. A. Ferreira, F. Chen and W. Cai, Wiley Interdiscip. Rev.: Nanomed. Nanobiotechnol., 2016, 8, 619-630.

628 R. Torres Martin de Rosales, R. Tavaré, R. L. Paul, M. Jauregui-Osoro, A. Protti, A. Glaria, G. Varma, I. Szanda and P. J. Blower, Angew. Chem., Int. Ed., 2011, 50, 5509-5513.

629 J. Pellico, I. Fernández-Barahona, M. Benito, Á. GaitánSimón, L. Gutiérrez, J. Ruiz-Cabello and F. Herranz, Nanomedicine, 2019, 17, 26-35.

630 J. Pellico, A. V. Lechuga-Vieco, E. Almarza, A. Hidalgo, C. Mesa-Nuñez, I. Fernández-Barahona, J. A. Quintana, J. Bueren, J. A. Enríquez, J. Ruiz-Cabello and F. Herranz, Sci. Rep., 2017, 7, 13242.

631 P. G. Jeelani, P. Mulay, R. Venkat and C. Ramalingam, Silicon, 2020, 12, 1337-1354.

632 Z. Li, J. C. Barnes, A. Bosoy, J. F. Stoddart and J. I. Zink, Chem. Soc. Rev., 2012, 41, 2590.

633 M. Manzano and M. Vallet-Regí, Adv. Funct. Mater., 2020, 30, 1902634.

634 R. Chakravarty, S. Goel, H. Hong, F. Chen, H. F. Valdovinos, R. Hernandez, T. E. Barnhart and W. Cai, Nanomedicine, 2015, 10, 1233-1246.

635 S. Nizzero, F. Li, G. Zhang, A. Venuta, C. Borsoi, J. Mai, H. Shen, J. Wolfram, Z. Li, E. Blanco and M. Ferrari, Acta Biomater., 2019, 97, 501-512.

636 L. Kramer, G. Winter, B. Baur, A. J. Kuntz, T. Kull, C. Solbach, A. J. Beer and M. Lindén, Nanoscale, 2017, 9, 9743-9753.

637 A. Al Faraj, B. Alotaibi, A. Pasha Shaik, K. Shamma, I. Al Jammaz and J. Gerl, Int. J. Nanomed., 2015, 6293.

638 H. Gao, X. Liu, W. Tang, D. Niu, B. Zhou, H. Zhang, W. Liu, B. Gu, X. Zhou, Y. Zheng, Y. Sun, X. Jia and L. Zhou, Nanoscale, 2016, 8, 19573-19580.

639 A. L. B. de Barros, K. S. de Oliveira Ferraz, T. C. S. Dantas, G. F. Andrade, V. N. Cardoso and E. M. B. de Sousa, Mater. Sci. Eng., C, 2015, 56, 181-188.

640 G. F. Andrade, J. A. Q. A. Faria, D. A. Gomes, A. L. B. de Barros, R. S. Fernandes, A. C. S. Coelho, J. A. Takahashi, A. da Silva Cunha and E. M. B. de Sousa, J. Sol-Gel Sci. Technol., 2018, 85, 369-381.
641 P. Dogra, N. L. Adolphi, Z. Wang, Y.-S. Lin, K. S. Butler, P. N. Durfee, J. G. Croissant, A. Noureddine, E. N. Coker, E. L. Bearer, V. Cristini and C. J. Brinker, Nat. Commun., 2018, 9, 4551.

642 A. Liberman, Z. Wu, C. V. Barback, R. Viveros, S. L. Blair, L. G. Ellies, D. R. Vera, R. F. Mattrey, A. C. Kummel and W. C. Trogler, ACS Nano, 2013, 7, 6367-6377.

643 S.-H. Cheng, D. Yu, H.-M. Tsai, R. A. Morshed, D. Kanojia, L.-W. Lo, L. Leoni, Y. Govind, L. Zhang, K. S. Aboody, M. S. Lesniak, C.-T. Chen and I. V. Balyasnikova, J. Nucl. Med., 2016, 57, 279-284.

644 X. Zhang, F. Chen, M. Z. Turker, K. Ma, P. Zanzonico, F. Gallazzi, M. A. Shah, A. R. Prater, U. Wiesner, M. S. Bradbury, M. R. McDevitt and T. P. Quinn, Biomaterials, 2020, 241, 119858.

645 Y. Fazaeli, M. A. Hosseini, M. Afrasyabi and P. Ashtari, Radiochim. Acta, 2019, 107, 157-164.

646 B. P. Burke, N. Baghdadi, A. E. Kownacka, S. Nigam, G. S. Clemente, M. M. Al-Yassiry, J. Domarkas, M. Lorch, M. Pickles, P. Gibbs, R. Tripier, C. Cawthorne and S. J. Archibald, Nanoscale, 2015, 7, 14889-14896.

647 F. Chen, S. Goel, S. Shi, T. E. Barnhart, X. Lan and W. Cai, Nano Res., 2018, 11, 4890-4904.

648 S. Goel, F. Chen, S. Luan, H. F. Valdovinos, S. Shi, S. A. Graves, F. Ai, T. E. Barnhart, C. P. Theuer and W. Cai, Adv. Sci., 2016, 3, 1600122.

649 S. Rojas, J. D. Gispert, C. Menchón, H. G. Baldoví, M. BuakiSogo, M. Rocha, S. Abad, V. M. Victor, H. García and J. R. Herance, J. Nanopart. Res., 2015, 17, 131.

650 P. A. Ellison, F. Chen, S. Goel, T. E. Barnhart, R. J. Nickles, O. T. DeJesus and W. Cai, ACS Appl. Mater. Interfaces, 2017, 9, 6772-6781.

651 F. Chen, H. F. Valdovinos, R. Hernandez, S. Goel, T. E. Barnhart and W. Cai, Acta Pharmacol. Sin., 2017, 38, 907-913.

652 E. Helal-Neto, S. S. Cabezas, F. Sancenón, R. MartínezMáñez and R. Santos-Oliveira, J. Pharm. Biomed. Anal., 2018, 153, 90-94.

653 L. T. M. Sá, S. Simmons, S. Missailidis, M. I. P. da Silva and R. Santos-Oliveira, J. Drug Targeting, 2013, 21, 427-434.

654 P. Rainone, B. Riva, S. Belloli, F. Sudati, M. Ripamonti, P. Verderio, M. Colombo, B. Colzani, M. C. Gilardi, R. M. Moresco and D. Prosperi, Int. J. Nanomed., 2017, 12, 3447-3461.

655 E. Boisselier and D. Astruc, Chem. Soc. Rev., 2009, 38, 1759. 656 J. Zhang, L. Mou and X. Jiang, Chem. Sci., 2020, 11, 923-936. 657 M. Grzelczak, J. Pérez-Juste, P. Mulvaney and L. M. LizMarzán, Chem. Soc. Rev., 2008, 37, 1783.

658 S. Same, A. Aghanejad, S. Akbari Nakhjavani, J. Barar and Y. Omidi, Bioimpacts, 2016, 6, 169-181.

659 J. L. Campbell, E. D. SoRelle, O. Ilovich, O. Liba, M. L. James, Z. Qiu, V. Perez, C. T. Chan, A. de la Zerda and C. Zavaleta, Biomaterials, 2017, 135, 42-52.

660 X. Sun, X. Huang, X. Yan, Y. Wang, J. Guo, O. Jacobson, D. Liu, L. P. Szajek, W. Zhu, G. Niu, D. O. Kiesewetter, S. Sun and X. Chen, ACS Nano, 2014, 8, 8438-8446. 
661 A. L. Tam, M. P. Melancon, M. Abdelsalam, T. A. Figueira, K. Dixon, A. McWatters, M. Zhou, Q. Huang, O. Mawlawi, K. Dunner, C. Li and S. Gupta, J. Biomed. Nanotechnol., 2016, 12, 296-307.

662 Y. Zhao, B. Pang, L. Detering, H. Luehmann, M. Yang, K. Black, D. Sultan, Y. Xia and Y. Liu, Mol. Imaging, 2018, 17, 153601211877582.

663 H. Li, L. Diaz, D. Lee, L. Cui, X. Liang and Y. Cheng, Radiol. Med., 2014, 119, 269-276.

664 M. Tian, W. Lu, R. Zhang, C. Xiong, J. Ensor, J. Nazario, J. Jackson, C. Shaw, K. A. Dixon, J. Miller, K. Wright, C. Li and S. Gupta, Mol. Imaging Biol., 2013, 15, 614-624.

665 H. Xie, Z. J. Wang, A. Bao, B. Goins and W. T. Phillips, Int. J. Pharm., 2010, 395, 324-330.

666 M. Pretze, N. P. van der Meulen, C. Wängler, R. Schibli and B. Wängler, J. Labelled Compd. Radiopharm., 2019, 62, 471-482.

667 L. E. Chilug, R. A. Leonte, M. E. B. Patrascu, A. C. Ion, C. S. Tuta, A. Raicu, G. Manda and D. Niculae, J. Radioanal. Nucl. Chem., 2017, 311, 1485-1493.

668 F. Silva, A. Zambre, M. P. C. Campello, L. Gano, I. Santos, A. M. Ferraria, M. J. Ferreira, A. Singh, A. Upendran, A. Paulo and R. Kannan, Bioconjugate Chem., 2016, 27, 1153-1164.

669 L. Karmani, V. Bouchat, C. Bouzin, P. Levêque, D. Labar, A. Bol, G. Deumer, R. Marega, D. Bonifazi, V. Haufroid, C. Michiels, V. Grégoire, O. Feron, S. Lucas, T. Vander Borght and B. Gallez, Nanomedicine, 2014, 9, 1923-1937.

670 L. Karmani, D. Labar, V. Valembois, V. Bouchat, P. G. Nagaswaran, A. Bol, J. Gillart, P. Levêque, C. Bouzin, D. Bonifazi, C. Michiels, O. Feron, V. Grégoire, S. Lucas, T. Vander Borght and B. Gallez, Contrast Media Mol. Imaging, 2013, 8, 402-408.

671 Y. Li, L. Zhao, X. Xu, N. Sun, W. Qiao, Y. Xing, M. Shen, M. Zhu, X. Shi and J. Zhao, J. Biomed. Nanotechnol., 2019, 15, 1201-1212.

672 F. Silva, L. Gano, M. P. Cabral Campello, R. Marques, I. Prudêncio, A. Zambre, A. Upendran, A. Paulo and R. Kannan, Dalton Trans., 2017, 46, 14572-14583.

673 M. Motiei, T. Dreifuss, T. Sadan, N. Omer, T. BlumenfeldKatzir, E. Fragogeorgi, G. Loudos, R. Popovtzer and N. BenEliezer, Chem. Lett., 2019, 48, 291-294.

674 N. Jiménez-Mancilla, G. Ferro-Flores, C. Santos-Cuevas, B. Ocampo-García, M. Luna-Gutiérrez, E. Azorín-Vega, K. Isaac-Olivé, M. Camacho-López and E. Torres-García, J. Labelled Compd. Radiopharm., 2013, 56, 663-671.

675 B. E. Ocampo-García, F. de, M. Ramírez, G. Ferro-Flores, L. M. De León-Rodríguez, C. L. Santos-Cuevas, E. MoralesAvila, C. A. de Murphy, M. Pedraza-López, L. A. Medina and M. A. Camacho-López, Nucl. Med. Biol., 2011, 38, 1-11.

676 O. J. Estudiante-Mariquez, A. Rodríguez-Galván, D. RamírezHernández, F. F. Contreras-Torres and L. A. Medina, Molecules, 2020, 25, 1982.

677 E. Morales-Avila, G. Ferro-Flores, B. E. Ocampo-García, L. M. De León-Rodríguez, C. L. Santos-Cuevas, R. GarcíaBecerra, L. A. Medina and L. Gómez-Oliván, Bioconjugate Chem., 2011, 22, 913-922.
678 A. Loiseau, J. Boudon, A. Oudot, M. Moreau, R. Boidot, R. Chassagnon, N. Mohamed Saïd, S. Roux, C. Mirjolet and N. Millot, Cancers, 2019, 11, 1962.

679 L. Song, S. Able, E. Johnson and K. A. Vallis, Nanotheranostics, 2017, 1, 232-243.

680 H.-W. Kao, Y.-Y. Lin, C.-C. Chen, K.-H. Chi, D.-C. Tien, C.C. Hsia, W.-J. Lin, F.-D. Chen, M.-H. Lin and H.-E. Wang, Nanotechnology, 2014, 25, 295102.

681 H. Mendoza-Nava, G. Ferro-Flores, F. de, M. Ramírez, B. Ocampo-García, C. Santos-Cuevas, E. Azorín-Vega, N. Jiménez-Mancilla, M. Luna-Gutiérrez and K. Isaac-Olivé, Mol. Imaging, 2017, 16, DOI: 10.1177/1536012117704768.

682 G. Ferro-Flores, B. Ocampo-García, C. Santos-Cuevas, F. María Ramírez, E. Azorín-Vega and L. MeléndezAlafort, Curr. Radiopharm., 2015, 8, 150-159.

683 A. Vilchis-Juárez, G. Ferro-Flores, C. Santos-Cuevas, E. Morales-Avila, B. Ocampo-García, L. Díaz-Nieto, M. LunaGutiérrez, N. Jiménez-Mancilla, M. Pedraza-López and L. Gómez-Oliván, J. Biomed. Nanotechnol., 2014, 10, 393-404.

684 E.-A. Salvanou, D. Stellas, C. Tsoukalas, B. Mavroidi, M. Paravatou-Petsotas, N. Kalogeropoulos, S. Xanthopoulos, F. Denat, G. Laurent, R. Bazzi, S. Roux and P. Bouziotis, Pharmaceutics, 2020, 12, 188.

685 A. F. Frellsen, A. E. Hansen, R. I. Jølck, P. J. Kempen, G. W. Severin, P. H. Rasmussen, A. Kjær, A. T. I. Jensen and T. L. Andresen, ACS Nano, 2016, 10, 9887-9898.

686 B. Pang, Y. Zhao, H. Luehmann, X. Yang, L. Detering, M. You, C. Zhang, L. Zhang, Z.-Y. Li, Q. Ren, Y. Liu and Y. Xia, ACS Nano, 2016, 10, 3121-3131.

687 Y. Zhao, D. Sultan, L. Detering, S. Cho, G. Sun, R. Pierce, K. L. Wooley and Y. Liu, Angew. Chem., Int. Ed., 2014, 53, 156-159.

688 M. Yang, D. Huo, K. D. Gilroy, X. Sun, D. Sultan, H. Luehmann, L. Detering, S. Li, D. Qin, Y. Liu and Y. Xia, ChemNanoMat, 2017, 3, 44-50.

689 Y. Zhao, D. Sultan, L. Detering, H. Luehmann and Y. Liu, Nanoscale, 2014, 6, 13501-13509.

690 J. Zhu, J. Chin, C. Wängler, B. Wängler, R. B. Lennox and R. Schirrmacher, Bioconjugate Chem., 2014, 25, 1143-1150.

691 K. R. Pulagam, K. B. Gona, V. Gómez-Vallejo, J. Meijer, C. Zilberfain, I. Estrela-Lopis, Z. Baz, U. Cossío and J. Llop, Molecules, 2019, 24, 3609.

692 Q. K. T. Ng, C. I. Olariu, M. Yaffee, V. F. Taelman, N. Marincek, T. Krause, L. Meier and M. A. Walter, Biomaterials, 2014, 35, 7050-7057.

693 R. Kamal, V. D. Chadha and D. K. Dhawan, Nanomedicine, 2018, 14, 1059-1071.

694 W. I. El-Ghareb, M. M. Swidan, I. T. Ibrahim, A. Abd ElBary, M. I. Tadros and T. M. Sakr, Int. J. Pharm., 2020, 586, 119514.

695 X. Shao, A. Agarwal, J. R. Rajian, N. A. Kotov and X. Wang, Nanotechnology, 2011, 22, 135102.

696 X. Shao, H. Zhang, J. R. Rajian, D. L. Chamberland, P. S. Sherman, C. A. Quesada, A. E. Koch, N. A. Kotov and X. Wang, ACS Nano, 2011, 5, 8967-8973.

697 Y. Zhang, Y. Zhang, L. Yin, X. Xia, F. Hu, Q. Liu, C. Qin and X. Lan, Contrast Media Mol. Imaging, 2017, 2017, 1-10. 
698 N. Eskandari, K. Yavari, M. Outokesh, S. Sadjadi and S. J. Ahmadi, J. Labelled Compd. Radiopharm., 2013, 56, 12-16.

699 C. Matea, T. Mocan, F. Tabaran, T. Pop, O. Mosteanu, C. Puia, C. Iancu and L. Mocan, Int. J. Nanomed., 2017, 12, 5421-5431.

700 X. Michalet, F. F. Pinaud, L. A. Bentolila, J. M. Tsay, S. Doose, J. J. Li, G. Sundaresan, A. M. Wu, S. S. Gambhir and S. Weiss, Science, 2005, 307, 538-544.

701 T. Tang, Y. Wei, Q. Yang, Y. Yang, M. J. Sailor and H.B. Pang, Nanoscale, 2019, 11, 22248-22254.

702 C. Tu, X. Ma, A. House, S. M. Kauzlarich and A. Y. Louie, ACS Med. Chem. Lett., 2011, 2, 285-288.

703 R. Mathur, N. Bag, R. Varshney, F. Hussain, A. Kaul, N. Kumari, R. Chauhan, S. Singh, S. Singh and A. K. Mishra, RSC Adv., 2016, 6, 13562-13571.

704 N. Bag, R. Mathur, F. Hussain, N. Indracanti, S. Singh, S. Singh, R. P. Chauhan, K. Chuttani and A. K. Mishra, Toxicol. Res., 2015, 4, 1416-1425.

705 M. Felber, M. Bauwens, J. M. Mateos, S. Imstepf, F. M. Mottaghy and R. Alberto, Chem. - Eur. J., 2015, 21, 6090-6099.

706 N. Bag, R. Mathur, S. Singh, F. Hussain, R. P. Chauhan, K. Chuttani and A. K. Mishra, MedChemComm, 2015, 6, 363-371.

707 A. Riedinger, T. Avellini, A. Curcio, M. Asti, Y. Xie, R. Tu, S. Marras, A. Lorenzoni, S. Rubagotti, M. Iori, P. C. Capponi, A. Versari, L. Manna, E. Seregni and T. Pellegrino, J. Am. Chem. Soc., 2015, 137, 15145-15151.

708 A. Rasekholghol, Y. Fazaeli, S. Moradi Dehaghi and P. Ashtari, J. Radioanal. Nucl. Chem., 2020, 324, 599-608, DOI: 10.1007/s10967-020-07102-y.

709 H. S. Sezgin, F. Z. Biber Muftuler, C. Ichedef, A. Yurt Kilcar, S. Teksoz, P. Unak and C. Harmansah, J. Radioanal. Nucl. Chem., 2017, 313, 291-297.

710 M. Sun, G. Sundaresan, P. Jose, L. Yang, D. Hoffman, N. Lamichhane and J. Zweit, J. Mater. Chem. B, 2014, 2, 4456-4466.

711 A. Kara, P. Ünak, C. Selçuki, Ö. Akça, E. İ. Medine and S. Sakarya, J. Radioanal. Nucl. Chem., 2014, 299, 807-813.

712 S. Wen, J. Zhou, K. Zheng, A. Bednarkiewicz, X. Liu and D. Jin, Nat. Commun., 2018, 9, 2415.

713 G. Tessitore, G. A. Mandl, M. G. Brik, W. Park and J. A. Capobianco, Nanoscale, 2019, 11, 12015-12029.

714 J. Rieffel, F. Chen, J. Kim, G. Chen, W. Shao, S. Shao, U. Chitgupi, R. Hernandez, S. A. Graves, R. J. Nickles, P. N. Prasad, C. Kim, W. Cai and J. F. Lovell, Adv. Mater., 2015, 27, 1785-1790.

715 H. J. Seo, S. H. Nam, H.-J. Im, J. Park, J. Y. Lee, B. Yoo, Y.-S. Lee, J. M. Jeong, T. Hyeon, J. Who Kim, J. S. Lee, I.-J. Jang, J.-Y. Cho, D. W. Hwang, Y. D. Suh and D. S. Lee, Sci. Rep., 2015, 5, 15685.

716 X. Cui, D. Mathe, N. Kovács, I. Horváth, M. Jauregui-Osoro, R. Torres Martin de Rosales, G. E. D. Mullen, W. Wong, Y. Yan, D. Krüger, A. N. Khlobystov, M. Gimenez-Lopez, M. Semjeni, K. Szigeti, D. S. Veres, H. Lu, I. Hernández,
W. P. Gillin, A. Protti, K. K. Petik, M. A. Green and P. J. Blower, Bioconjugate Chem., 2016, 27, 319-328.

717 J. Gallo, I. S. Alam, J. Jin, Y.-J. Gu, E. O. Aboagye, W.-T. Wong and N. J. Long, Dalton Trans., 2014, 43, 5535.

718 X. Yang, H. Hong, J. J. Grailer, I. J. Rowland, A. Javadi, S. A. Hurley, Y. Xiao, Y. Yang, Y. Zhang, R. J. Nickles, W. Cai, D. A. Steeber and S. Gong, Biomaterials, 2011, 32, 4151-4160.

719 E. Christensen, J. R. Henriksen, J. T. Jørgensen, Y. Amitay, H. Schmeeda, A. A. Gabizon, A. Kjær, T. L. Andresen and A. E. Hansen, Int. J. Nanomed., 2018, 13, 7647-7656.

720 H. Savolainen, A. Volpe, A. Phinikaridou, M. Douek, G. Fruhwirth and R. T. M. de Rosales, Nanotheranostics, 2019, 3, 255-265.

721 K. O. Jung, T. J. Kim, J. H. Yu, S. Rhee, W. Zhao, B. Ha, K. Red-Horse, S. S. Gambhir and G. Pratx, Nat. Biomed. Eng., 2020, 1-10.

722 S. J. Wang, W. S. Huang, C. M. Chuang, C. H. Chang, T. W. Lee, G. Ting, M. H. Chen, P. M. H. Chang, T. C. Chao, H. W. Teng, Y. Chao, Y. M. Chen, T. P. Lin, Y. J. Chang, S. J. Chen, Y. R. Huang and K. L. Lan, EJNMMI Res., 2019, 9, 46.

723 K. J. Harrington, S. Mohammadtaghi, P. S. Uster, D. Glass, A. M. Peters, R. G. Vile and J. S. W. Stewart, Clin. Cancer Res., 2001, 7, 243-254.

724 J. W. Nichols and Y. H. Bae, J. Controlled Release, 2014, 190, 451-464.

725 U. Prabhakar, H. Maeda, R. K. Jain, E. M. Sevick-Muraca, W. Zamboni, O. C. Farokhzad, S. T. Barry, A. Gabizon, P. Grodzinski and D. C. Blakey, Cancer Res., 2013, 73, 2412-2417.

726 T. Lammers, L. Y. Rizzo, G. Storm and F. Kiessling, Clin. Cancer Res., 2012, 18, 4889-4894.

727 M. A. Miller, S. Arlauckas and R. Weissleder, Nanotheranostics, 2017, 1, 296-312.

728 M. F. Kircher, S. S. Gambhir and J. Grimm, Nat. Rev. Clin. Oncol., 2011, 8, 677-688.

729 S. Behzadi, V. Serpooshan, W. Tao, M. A. Hamaly, M. Y. Alkawareek, E. C. Dreaden, D. Brown, A. M. Alkilany, O. C. Farokhzad and M. Mahmoudi, Chem. Soc. Rev., 2017, 46, 4218-4244.

730 P. Bhatnagar, Z. Li, Y. Choi, J. Guo, F. Li, D. Y. Lee, M. Figliola, H. Huls, D. A. Lee, T. Zal, K. C. Li and L. J. N. Cooper, Integr. Biol., 2012, 5, 231-238.

731 S. B. Lee, S. B. Ahn, S.-W. Lee, S. Y. Jeong, Y. Ghilsuk, B.-C. Ahn, E.-M. Kim, H.-J. Jeong, J. Lee, D.-K. Lim and Y. H. Jeon, NPG Asia Mater., 2016, 8, e281-e281.

732 P. Bhatnagar, M. Alauddin, J. A. Bankson, D. Kirui, P. Seifi, H. Huls, D. A. Lee, A. Babakhani, M. Ferrari, K. C. Li and L. J. N. Cooper, Sci. Rep., 2014, 4, 1-6.

733 M. Yao, X. Shi, C. Zuo, M. Ma, L. Zhang, H. Zhang, X. Li, G.-Y. Yang, Y. Tang and R. Wu, ACS Appl. Mater. Interfaces, 2020, 12(34), 37885-37895, DOI: 10.1021/acsami.0c10500.

734 N. Kotagiri, M. L. Cooper, M. Rettig, C. Egbulefu, J. Prior, G. Cui, P. Karmakar, M. Zhou, X. Yang, G. Sudlow, L. Marsala, C. Chanswangphuwana, L. Lu, L. Habimana-Griffin, 
M. Shokeen, X. Xu, K. Weilbaecher, M. Tomasson, G. Lanza, J. F. DiPersio and S. Achilefu, Nat. Commun., 2018, 9, 275.

735 T. M. Shaffer, E. C. Pratt and J. Grimm, Nat. Nanotechnol., 2017, 12, 106-117.

736 N. Kotagiri, G. P. Sudlow, W. J. Akers and S. Achilefu, Nat. Nanotechnol., 2015, 10, 370-379.

737 D. Duan, H. Liu, Y. Xu, Y. Han, M. Xu, Z. Zhang and Z. Liu, ACS Appl. Mater. Interfaces, 2018, 10, 5278-5286.

738 R. Tang, A. Zheleznyak, M. Mixdorf, A. Ghai, J. Prior, K. C. L. Black, M. Shokeen, N. Reed, P. Biswas and S. Achilefu, ACS Nano, 2020, 14, 4255-4264.

739 S. Kavadiya and P. Biswas, J. Nucl. Med., 2019, 60, 702-709.
740 D. Ni, C. A. Ferreira, T. E. Barnhart, V. Quach, B. Yu, D. Jiang, W. Wei, H. Liu, J. W. Engle, P. Hu and W. Cai, J. Am. Chem. Soc., 2018, 140, 14971-14979.

741 B. Yu, D. Ni, Z. T. Rosenkrans, T. E. Barnhart, H. Wei, C. A. Ferreira, X. Lan, J. W. Engle, Q. He, F. Yu and W. Cai, Adv. Mater., 2019, 31, 1904894.

742 R. Weissleder, M. Nahrendorf and M. J. Pittet, Nat. Mater., 2014, 13, 125-138.

743 W. J. M. Mulder, J. Ochando, L. A. B. Joosten, Z. A. Fayad and M. G. Netea, Nat. Rev. Drug Discovery, 2019, 18, 553-566.

744 C. Wang, B. I. Leach, D. Lister, S. R. Adams, H. Xu, C. K. Hoh, P. McConville, J. Zhang, K. Messer and E. Ahrens, J. Nucl. Med., 2020, DOI: 10.2967/jnumed.120.255273. 